LBL-37290

UC-414

\title{
Development of Radiation Detectors Based on Hydrogenated Amorphous Silicon and Its Alloys
}

\author{
Wan-Shick Hong \\ Ph.D. dissertation \\ Department of Materials Science and Mineral Engineering \\ University of California at Berkeley \\ and \\ Physics Division \\ Lawrence Berkeley Laboratory \\ University of California \\ Berkeley, CA 94720
}

April 1995

DISTRIBUTION OF THIS DOCUMENT IS UNLIMITED

This work was supported by the Director, Office of Energy Research, Office of High Energy and Nuclear Physics, Division of High Energy Physics of the U.S.Department of Energy under Contract No.DE-AC03-76SF00098. 
<smiles>[3H]</smiles>

, 


\section{DISCLAIMER}

This report was prepared as an account of work sponsored by an agency of the United States Government. Neither the United States Government nor any agency thereof, nor any of their employees, make any warranty, express or implied, or assumes any legal liability or responsibility for the accuracy, completeness, or usefulness of any information, apparatus, product, or process disclosed, or represents that its use would not infringe privately owned rights. Reference herein to any specific commercial product, process, or service by trade name, trademark, manufacturer, or otherwise does not necessarily constitute or imply its endorsement, recommendation, or favoring by the United States Government or any agency thereof. The views and opinions of authors expressed herein do not necessarily state or reflect those of the United States Government or any agency thereof. 


\section{DISCLAIMER}

Portions of this document may be illegible in electronic image products. Images are produced from the best available original document. 
Development of Radiation Detectors Based on Hydrogenated Amorphous Silicon and Its Alloys

\author{
by \\ Wan-Shick Hong \\ B.S. (Seoul National University) 1988 \\ M.S. (University of California at Berkeley) 1991
}

A dissertation submitted in partial satisfaction of the requirements for the degree of

Doctor of Philosophy

in

Engineering - Materials Science

and Mineral Engineering

in the

GRADUATE DIVISION

of the

UNIVERSITY of CALIFORNIA at BERKELEY

Committee in charge:

Professor Victor Perez-Mendez, Co-chair

Professor Lutgard C. De Jonghe, Co-chair

Professor Selig N. Kaplan

Professor Eicke R. Weber 


\title{
Development of Radiation Detectors Based on Hydrogenated Amorphous Silicon and Its Alloys
}

\author{
Copyright @ 1995 \\ by \\ Wan-Shick Hong
}

The United States Department of Energy has the right to use this dissertation for any purpose whatsoever including the right to reproduce all or any part thereof. 


\author{
Abstract \\ Development of Radiation Detectors Based on \\ Hydrogenated Amorphous Silicon and Its Alloys \\ by \\ Wan-Shick Hong
}

\begin{abstract}
Doctor of Philosophy in Materials Science and Mineral Engineering University of California at Berkeley

Professor Victor Perez-Mendez, Co-chair

Professor Lutgard C. DeJonghe, Co-chair
\end{abstract}

Hydrogenated amorphous silicon and related materials have been applied to radiation detectors, utilizing their good radiation resistance and the feasibility of making deposits over a large area at low cost. Effects of deposition parameters on various material properties of a-Si:H have been studied to produce a material satisfying the requirements for specific detection application.

Thick $(\sim 50 \mu \mathrm{m})$, device quality a-Si:H p-i-n diodes for direct detection of minimum ionizing particles have been prepared with low internal stress by a combination of low temperature growth, He-dilution of silane, and post annealing. The structure of the new film contained voids and tiny crystalline inclusions and was different from the one observed in conventional a-Si:H. Deposition on patterned substrates was attempted as an alternative to controlling deposition parameters to minimize substrate bending and delamination of thick a-Si:H films. Growth on an inversed-pyramid pattern reduced the substrate bending by a factor of 3 4 for the same thickness film. 
Thin $(0.1 \sim 0.2 \mu \mathrm{m})$ films of a-Si:H and a-Si:C:H have been applied to microstrip gas chambers to control gain instabilities due to charges on the substrate. Light sensitivity of the a-Si:H sheet resistance was minimized and the surface resistivity was successfully controlled in the range of $10^{12} \sim 10^{17} \Omega / \Omega$ by carbon alloying and boron doping. Performance of the detectors with boron-doped a-Si:C:H layers was comparable to that of electronic-conducting glass.

Hydrogen dilution of silane has been explored to improve electrical transport properties of a-Si:H material for high speed photo-detectors and TFT applications. Various electrical properties of the hydrogen-diluted a-Si:H showed factors of 3 10 improvements at the expense of increased residual stress. The optimal material characteristics for TFT application appeared at deposition conditions corresponding to the onset of crystalline formation, indicating that the enhancement of the electrical properties is due to the superior amorphous matrix not to the presence of crystalline phase.

Material properties of the a-Si:H based materials can be easily adapted to the characteristics required for individual applications by modifying fabrication conditions and procedures. This asset, in addition to the advantages of large area capability and radiation hardness, makes a-Si:H an attractive candidate for radiation detection applications.

Committee in charge: Prof. V. Perez-Mendez, Co-chair:

Prof. L. C. De Jonghe, Co-chair:

Prof. S. N. Kaplan

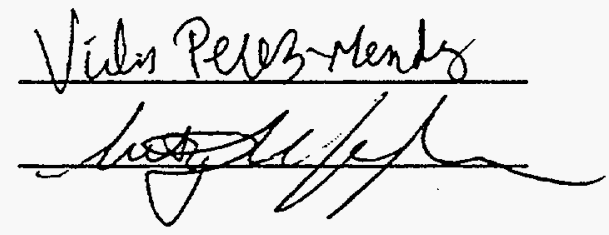

Prof. E. R. Weber 
To my grandfather 


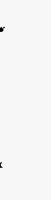




\section{CHAPTER 2 BACKGROUND}

2.1 BASIC FEATURES OF HYDROGENATED AMORPHOUS SILICON ............ 4

2.1.1 Microstructure ...................................................... 4

2.1.2 Band Structure and Electrical Properties ............................... 7

2.1.3 Substitutional Doping …........................................... 10

2.1.4 Electronic Transport ................................................. 14

2.1.5 Deposition Method ................................................ 16

2.2 RADIATION DETECTION SCHEMES _........................................ 19

2.2.1 Radiation Detection Principles ............................................ 19

2.2.2 Amorphous Silicon Detectors ....................................... 22

2.3 RESIDUAL STRESSES IN THIN FILMS _..................................... 24

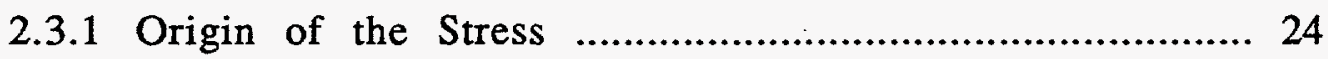

2.3.2 Mechanics and Measurements of stress in Thin Films on Substrates 26

2.3.3 Delamination and Spallation ............................................. 29

CHAPTER 3 THICK $(\sim 50 \mu \mathrm{m})$ a-Si:H LAYERS FOR DIRECT DETECTION OF MINIMUM IONIZING PARTICLES

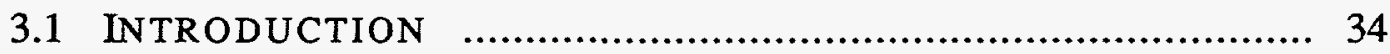

3.1.1 Fully-Depleted Detectors …........................................... 35

3.1.2 Minimum Ionizing Particles ......................................... 37

3.1.3 Effect of Dangling Bonds ............................................. 38

3.1.4 Residual Stress in a-Si:H Film …...................................... 39

3.1.5 Relationship between Stress and Dangling Bond Density ............ 41

3.1.6 Deposition Rate .................................................. 43 
3.2 FIELD PROFILE TAILORING _............................................ 45

3.2.1 Calculation of Electron Loss ........................................ 46

3.2.2 Measurement Results ............................................... 50

3.3 GROWTH OF THICK LAYERS …........................................ 53

3.3.1 Increased Deposition Rate .......................................... 53

3.3.2 Heat Treatment for Low Defect Density ................................ 56

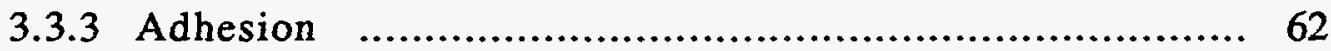

3.4 REDUCTION OF RESIDUAL STRESS .........................................6 68

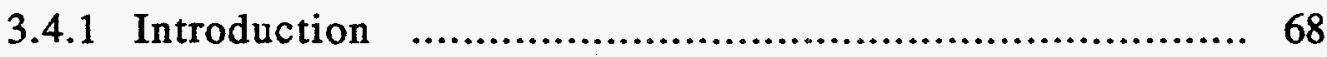

3.4.2 Experimental Procedures …........................................ 69

3.4.3 Optimization of the Deposition Conditions ............................ 70

3.4.4 IR Absorption and Raman Scattering ................................ 75

3.4.5 Microstructures of the Conventional a-Si:H and the New Films .... 79

3.5 SUSBTRATE PATTERNING FOR INCREASED MECHANICAL STABILITY .... 84

3.5.1 Square Island Stucture .......................................... 84

3.5.2 Inverted Pyramid Structure ........................................... 90

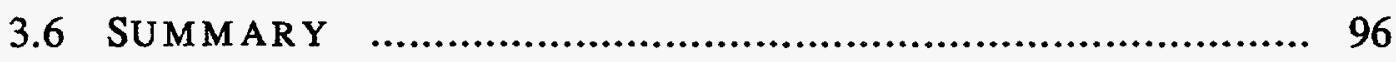

\section{CHAPTER 4 MICROSTRIP / MICROGAP GAS CHAMBERS}

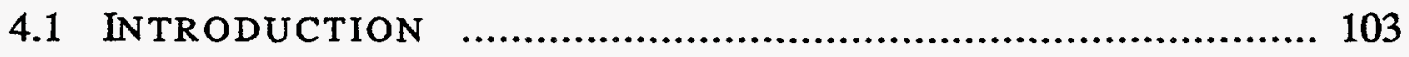

4.1.1 Historical Background ................................................ 103

4.1.2 Problems in Operating the MSGCs ................................. 105

4.1.3 Application of a-Si:H and Its Alloys to MSGCs ...................... 107

4.2 EXPERIMENTAL PROCEDURES ........................................... 108

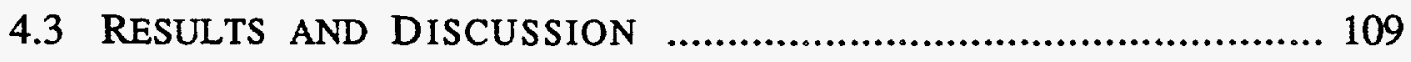

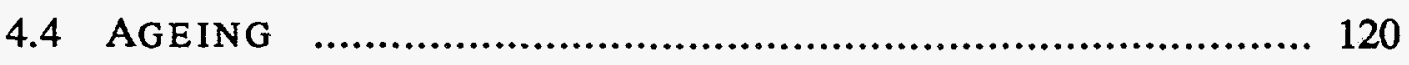

4.5 MICROGAP CHAMBER (MGC) …...................................... 122 
CHAPTER 5 THIN $(<2 \mu \mathrm{m})$ a-Si:H LAYERS FOR READOUT AND OTHER APPLICATIONS

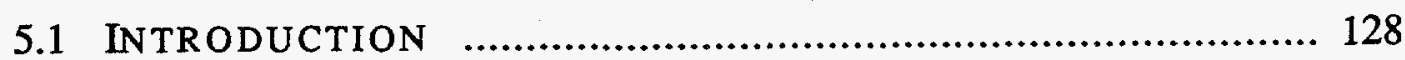

5.2 HYdROGEN DILUTION FOR BETTER ELECTRICAL PROPERTIES ........... 129

5.2.1 Introduction ................................................. 129

5.2.2 Experimental Procedures ............................................ 130

5.2 .3 Results ......................................................... 133

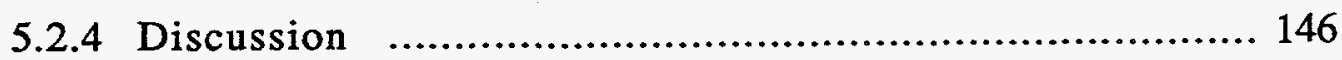

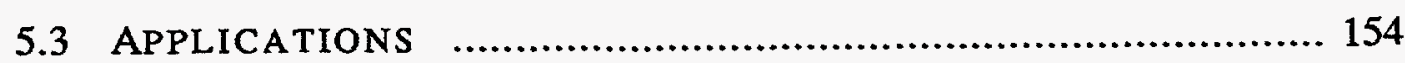

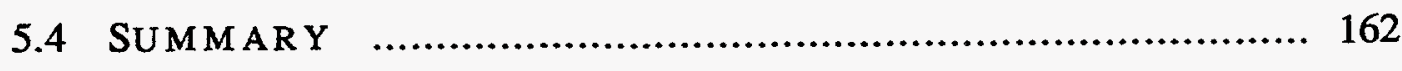

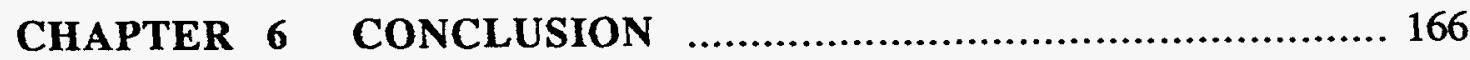




\section{Acknowledgements}

First of all, I thank my LORD, God Almighty, who allowed me to finish my Ph.D. work and helped me to focus on Him all the time including times of trouble. It has been more valuable to have a fellowship with Him than to achieve my thesis goal itself.

My special thanks go to Prof. Victor Perez-Mendez for his unreasonable-at-firstbut-fascinating-after-second-thought ideas and warm-hearted guidance. I am also grateful to Prof. Lutgard De Jonghe who first accepted me to Berkeley and helped all the academic matters. I owe thanks to Prof. Selig Kaplan for his continuing encouragement and valuable comments. I also thank Prof. Eicke Weber and Prof. Ron Gronsky for helping me with tremendous knowledge on device physics from classes to qualifying exam to dissertation.

I would also like to thank Prof. Juan Carlos Delgado at Univ. of Barcelona, Spain, for his encouragement and friendship. I thank all members of our group, especially Dr. Gyuseong Cho and Tao Jing, for their assistance in adjusting to the new world of amorphous silicon and photolithography. In addition, I owe a lot to the staff members of the Microelectronics Lab on campus for their cordial and timely assistance. Prof. Hwan Kim and other professors in the Dept. of Inorganic Materials Engineering, Seoul National University, also deserve special thanks since they constructed my background as a materials scientist and granted me an opportunity to study abroad.

I extend my gratitude to all my spiritual family in Berkland Baptist Church, who have prayed for me and helped me to stand straight in this world. Finally, I thank my parents and sisters in Korea and my wife, Jung Min, for their support and unceasing love.

This work was supported by the Director, Office of Energy Research, Office of High Energy and Nuclear Physics, Division of High Energy Physics of the U.S.Department of Energy under Contract No. DE-AC03-76SF00098. 


\section{CHAPTER 1. INTRODUCTION}

Research in amorphous semiconductors has been directed towards how the disorder of the non-crystalline structure relates to their unique electronic properties. Interest in the amorphous semiconductors developed with chalcogenide glasses, and then amorphous silicon (a-Si) began to flourish as a derivative of crystalline silicon. In early days, amorphous silicon without hydrogen was prepared by sputtering or by thermal evaporation. However, this unhydrogenated material has a very high defect density, which prevents this material from being useful for electronic devices due to difficulty in doping, and poor electrical characteristics.

Hydrogenated amorphous silicon (a-Si:H) was first made by Chittick and coworkers using plasma-enhanced chemical vapor deposition (PECVD) technique.[1] Silane $\left(\mathrm{SiH}_{4}\right)$ gas molecules are decomposed by plasma and deposit on heated substrates. Hydrogenation greatly improves the photoconductivity and other desirable characteristics. Combination of the disordered atomic structure and the presence of hydrogen in the amorphous silicon gives different phenomena from those in the crystalline counterpart. Hydrogen is now recognized as an essential component of the a-Si:H films that is responsible for passivating defects.

A breakthrough in the development of a-Si:H was the success in substitutional ntype or p-type doping by Spear and LeComber.[2] Since then, a-Si:H is gaining increasing use in photovoltaic solar cells, facsimile heads, liquid crystal display drivers and so forth. The special attribute of a-Si:H is the ability to deposit the material inexpensively over large areas and the higher resistance to radiation damage compared to the crystalline silicon. These assets drew attention to a-Si:H as a possible alternative for radiation detector material for high energy physics experiments, medical imaging and life science studies.

Thin $(1 \sim 2 \mu \mathrm{m})$ a-Si:H layers have been studied extensively for $\mathrm{x}$-ray detectors.[3, 4] Thin a-Si:H p-i-n diode can also be coupled to an appropriate scintillator for $\mathrm{x}$-ray or 
charged particle detection.[5] Thick ( $50 \mu \mathrm{m})$ layers are required for direct detection of single particles, especially minimum ionizing particles.[6, 7] This thesis focuses on utilization of a-Si:H in various types of radiation detectors. Modification of material properties, such as electronic transport characteristic and residual stress, to fit this material to specific applications is discussed.

In Chapter 2, background information is briefly reviewed. The topics include material characteristics of a-Si:H, principles of radiation detection, and mechanics of thin films.

In Chapter 3, preparation and operation of thick $(\sim 50 \mu \mathrm{m})$ a-Si:H detectors for minimum ionizing particle detection are discussed. Nature of the radiation and problems in making thick films are addressed. Solution to each obstacle is discussed, and finally, a new method for fabricating good quality films with increased mechanical stability is proposed, consolidating knowledge acquired from various approaches. The main goal is to achieve a lowered residual stress in the film to increase mechanical stability while not sacrificing the electrical properties too much. A microstructural model is derived based on various characterization techniques. A new configuration of the detector diodes for operating at a reduced bias voltage is also proposed.

In Chapter 4, utilization of amorphous silicon as a resistive layer to improve long term stability of microstrip detectors is studied. Two important roles of the a-Si:H coating are to control surface resistivity to prevent charge-up and to protect electrode strips against ageing. Alloying of a-Si: $\mathrm{H}$ with carbon and doping with boron are done to obtain suitable sheet resistance and minimized light sensitivity. Performance of the microstrip detectors coated with doped a-Si:C:H is compared with detectors having surface layers of other kinds.

Chapter 5 deals with thin amorphous silicon layers for applications to readout circuit and TFT amplifiers that can be integrated with pixel detector arrays. In order to improve the electronic properties, such as carrier mobility and defect density, hydrogen 
dilution of silane is attempted. Depending on the deposition conditions, introducing extra hydrogen to the plasma leads to formation of a microcrystalline phase. Changes in the material properties with deposition parameters and hydrogen content are described. Applications of the 2-dimensional pixel array made of the high quality amorphous or microcrystalline silicon are discussed as well.

All the topics discussed in Chapters $2 \sim 5$ are summarized in Chapter 6 .

\section{References}

[1] R.C.Chittick, J.H.Alexander and H.F.Sterling, J. Electrochem. Soc., 11677 (1969)

[2] W.E.Spear and P.G.LeComber, Solid State Commun., 171193 (1975)

[3] G.-P. Wei, H.Okamoto and Y.Hamakawa, Jap. J. Appl. Phys., 241105 (1985)

[4] R.A.Street, S.Nelson, L.E.Antonuk and V.Perez-Mendez, Mat. Res. Soc. Symp. Proc., 192441 (1990)

[5] T.Jing, C.A.Goodman, G.Cho, J.S.Drewery, W.S.Hong, H.Lee, S.N.Kaplan, A.Mireshghi, V.Perez-Mendez and D.Wildermuth, IEEE Trans. Nucl. Sci., NS-41, 903 (1994)

[6] V.Perez-Mendez, S.N.Kaplan, G.Cho, I.Fujieda, S.Qureshi, W.Ward and R.A.Street, Nucl. Instr. Meth., A 273127 (1988)

[7] S.N.Kaplan, J.Morel, V.Perez-Mendez and R.A.Street, IEEE Trans. Nucl. Sci., NS33351 (1986) 


\section{CHAPTER 2. BACKGROUND}

\subsection{BaSiC FEATURES OF HYDROGENATED AMORPhOUS SILICON}

\subsubsection{Microstructure}

The disorder of the atomic structure is the main feature that distinguishes amorphous from crystalline materials. Bloch's theorem, which describes the electrons and holes by periodic wavefunctions, is not suitable for relating the atomic structure and electronic properties of this material because of the lack of long-range periodicity. Instead, the chemical bonding between the atoms, especially the short range bonding interactions, has more importance.[1]

Figure 2.1 compares the atomic configuration of amorphous and crystalline silicon. The atomic structure of a-Si:H is represented by a 3-dimensional continuous random network whereas the crystalline silicon forms a diamond structure. X-ray and electron diffraction measurements show that a high degree of short-range order is retained in a-Si:H because of the chemical bonding resulting in nearly equal bond length and bond angles as in crystalline silicon.[2]

The disorder is represented by the radial distribution function (RDF), which is the probability of finding an atom at a distance, $R$, from another atom. The amorphous material has the same short range order as the crystal but lacks the long range order. The material properties of amorphous silicon are similar to its crystalline counterpart because they share the same local order. However, amorphous silicon forms an overconstrained network since its intrinsic coordination number is 4 while the most preferred average coordination number of a three-dimensional random network is $\sqrt{6}=2.45$.[3] For an overconstrained material most of the strain is relieved by distortion of the bond angles. Thus the a-Si:H exhibits a $\pm 10^{\circ}$ spread from the optimal tetrahedral bond angle of $109.5^{\circ}$. 


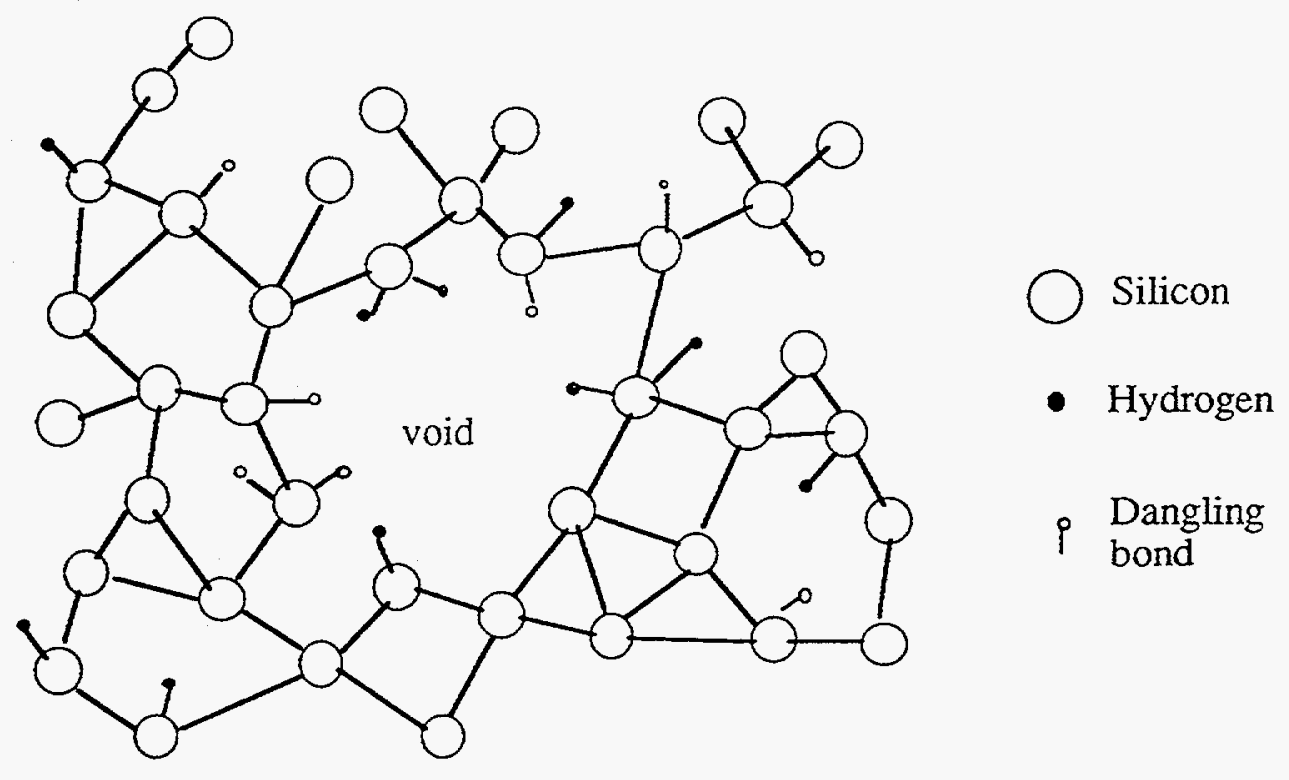

(a)

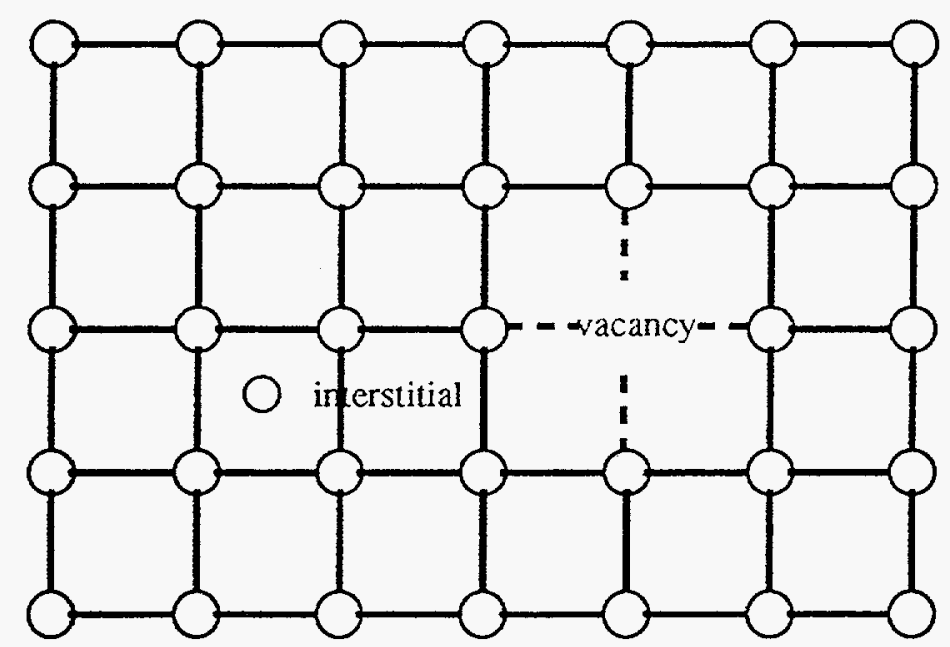

(b)

Figure 2.1. Atomic configurations of (a) amorphous, and (b) crystalline silicon 


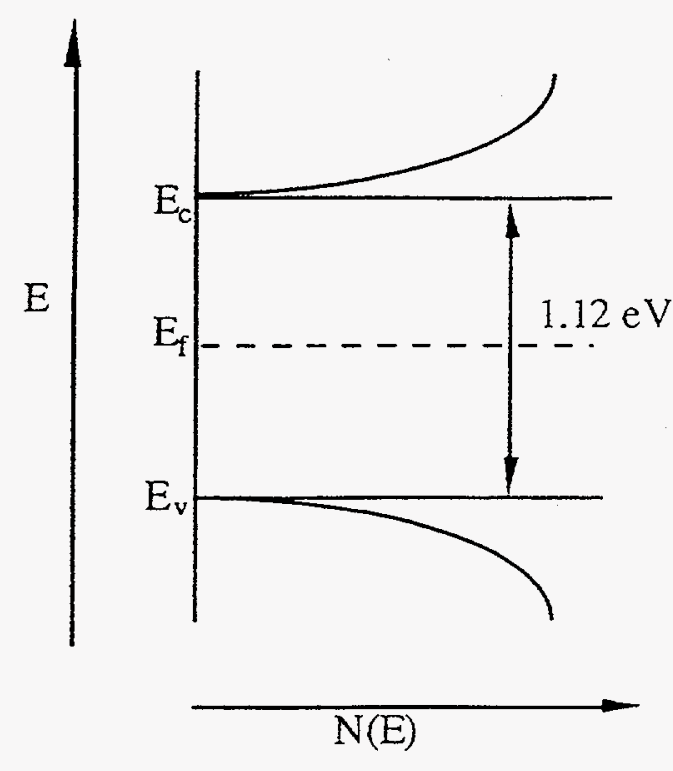

(a)

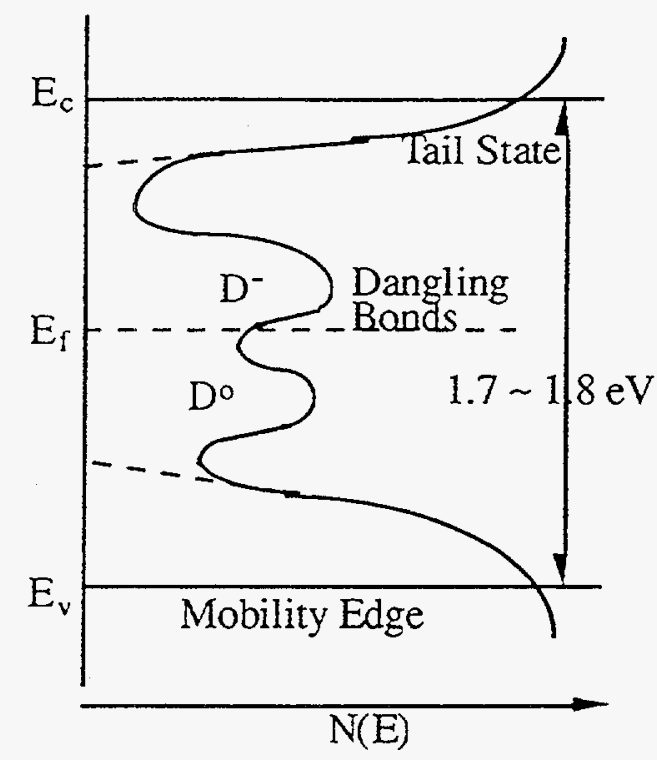

(b)

Figure 2.2. Energy band structures of (a) crystalline and (b) amorphous silicon

The preservation of the local order leads to a band gap of a-Si:H comparable to its crystalline counterpart. However, the disorder represented by deviations in the bond lengths and bond angles broadens the electron distribution of states and causes electron and hole localization as well as strong scattering of carriers. Structural defects such as broken bonds have corresponding electronic states deep in the band gap. The resulting energy band structure is depicted in Fig. 2.2 .

A defect is defined as any atom which is out of place in a crystal - typical examples are vacancies, interstitials, and dislocations. However, the definition of a defect has to be modified in the amorphous material, since there are no strictly defined lattice sites. The only specific structural feature of a random network is the coordination of an atom to its neighbor. Thus the elementary defects of an amorphous semiconductor are the 
coordination defect, when an atom has too many or too few bonds, and microvoids between clusters.

The broken bonds (or dangling bonds) are the most important defects in a-Si:H, because they not only control the position of the Fermi energy but also the transport properties of the material. In addition, they act as traps and recombination centers. Furthermore, in intrinsic a-Si:H the defect centers release charges which are swept out by the electric field thus producing space charge in the material which then shapes the electric field inside the sample. Alloying with any element from other columns in the periodic table, e.g. hydrogen, lowers the average coordination and tends to reduce the local bond distortion and defects.[4] As hydrogen binds to dangling bonds and creates a $\mathrm{Si}-\mathrm{H}$ bond that is stronger than the $\mathrm{Si}-\mathrm{Si}$ bond, hydrogenation enhances the electronic property of a$\mathrm{Si}: \mathrm{H}$ largely. When the atomic hydrogen is introduced, it reduces the defect states from $\sim 10^{19}$ to $\sim 10^{15} \mathrm{~cm}^{-3}$ in the gap between the conduction and valence bands. Atomic hydrogen concentration of about $10 \%$ is known to be the optimum condition to make a device quality material.[5]

\subsubsection{Band Structure and Electrical Properties}

The Bloch potential, $V(r)$, in an amorphous semiconductor is not periodic and this disordering effect results in scattering the electron from one Bloch state to another. The strong scattering causes a large uncertainty in the electron momentum and, through the uncertainty principle, the uncertainty in wavevector, $\Delta k$, is similar to the magnitude of $k$. Thus the energy bands are no longer described by the $E-k$ dispersion relations, but by a density of state distribution, $N(E)$.

As discussed in the previous section, the similarity in short range order between amorphous and crystalline silicon leads to a similar overall electronic structure. The amorphous phase has a band gap energy comparable to that of the crystalline phase. 
However, the disorder represented by deviations in the bond length and bond angles broadens the electron distribution of states and causes electron and hole localization as well as strong scattering of the carriers. As a result, the conduction and valence bands in amorphous silicon have deeply penetrating band tails into the forbidden gap.[6] Although there is no precise location of the gap because the band tail density of states decays continuously with energy, the band gap can be defined in two ways: optical transition and mobility edge.

\section{(1) Optical gap (Tauc gap):}

Optical band-to-band transitions are responsible for the main absorption band and are the primary measure of the band gap energy.[7] It is assumed that the density of states increases as a power law of energy, $\left(E-E_{g}\right)^{n}$, from an extrapolated band edge, where $E_{g}$ is the band gap defined by the separation of the two extrapolated band edges and $n$ is a power constant. The absorption near the gap is given from the joint density of states by

$$
\hbar \omega \cdot \alpha(\hbar \omega)=A\left(\hbar \omega-E_{g}\right)^{\left(n_{c}+n_{v}+1\right)}
$$

where $A$ is a matrix whose elements are independent of energy and the subscripts $c$ and $v$ represent conduction and valence band, respectively. For parabolic band edges, $n_{c}=n_{v}=$ $1 / 2$

$$
[\hbar \omega \cdot \alpha(\hbar \omega)]^{1 / 2}=A^{\prime}\left(\hbar \omega-E_{g}\right)
$$

This relation provides a simple procedure to extract $E_{g}$ from the measurement of $\alpha(\hbar \omega)$, and the $E_{g}$ can be defined in terms of an extrapolation of the density of states such that the absorption coefficient, $\alpha(\hbar \omega)$, becomes zero. The plot of $\alpha(\hbar \omega)^{1 / 2}$ as a function of energy is called the Tauc plot and is useful in comparing the band gaps of a-Si:H prepared in different ways. 


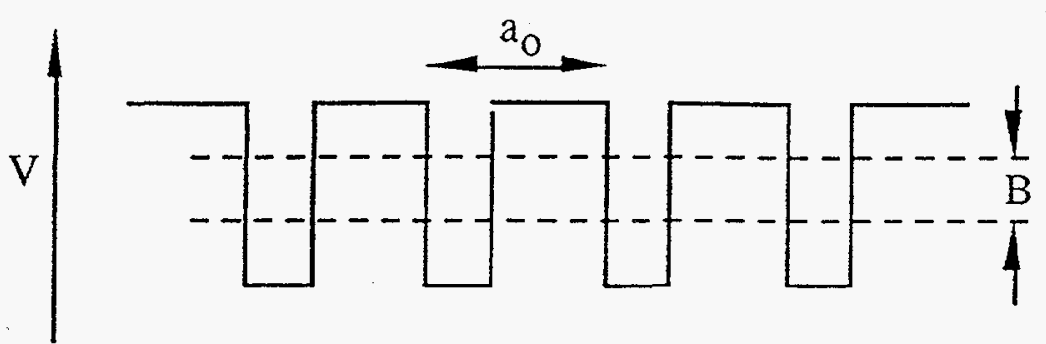

(a) crystalline

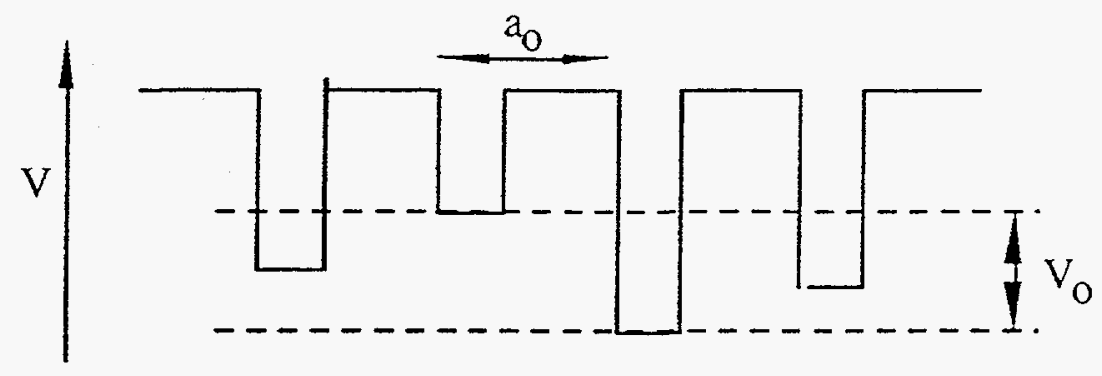

(b) amorphous

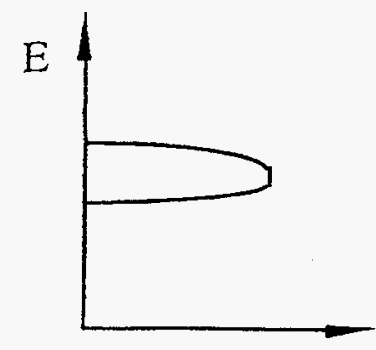

$N(E)$

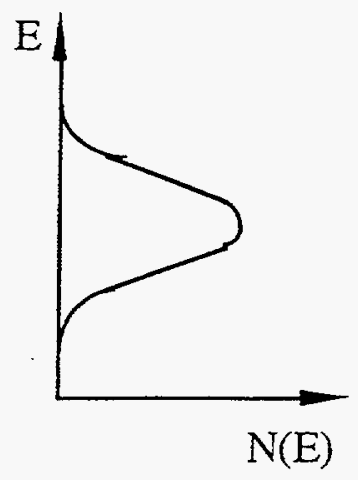

Figure 2.3. Schematic diagrams of potential wells in the Anderson model and the density of states. (a) a crystalline lattice with periodic potential, (b) an amorphous network with random potential. $V_{o}$ is the disorder potential and $B$ is the bandwidth when $V_{0}$ vanishes. [4]

(2) Mobility gap:

The other definition of the band gap is the energy separation of the valence and conduction band mobility edges. An increasing disorder potential causes electron localization in which the wavefunction is confined to a small volume rather than extended.

Anderson's theory of localization predicted that, for a carrier moving in a potential well, all states would be localized if $V_{o} / B$ exceeds a critical value where $V_{o}$ is the extent of the random potential and $B$ is the bandwidth when $V_{o}$ vanishes.[8] The Anderson model of the potential well is illustrated in Fig. 2.3. In a-Si:H, the disorder potential is insufficient to meet the Anderson criterion. Mott showed that, even when the value of 
$V_{o} / B$ was smaller than the critical value, some of the states are localized and are separated from the extended states by a sharp energy $E_{c}$.[9] At zero temperature, only electrons above $E_{c}$ are mobile and contribute to the conduction. In the band tail states, the density of states becomes so low that the electron wave function does not overlap between the states, and the localized states lie at the band edges. Hence electrons and holes which are mostly located in the tail states cannot move as freely as in the conduction or valence band and the transport of electrons or holes is characterized by ranges in values of mobilities depending on the energy state. The position of the mobility edge is an intrinsic property of the continuous random network and the mobility gap, which is defined by the separation of conduction and valence band mobility edges, is generally larger than the Tauc gap.

\section{(3) Gap states:}

The dangling bonds create energy states in the middle of the forbidden gap between the conduction and valence bands. The density of states has a broad peak which can be approximated by a gaussian distribution with a width of a few tenths of an $\mathrm{eV}$. A dangling bond can have three states; empty $\left(\mathrm{D}^{+}\right)$, singly occupied($\left(\mathrm{D}^{\circ}\right)$, doubly occupied($\left(\mathrm{D}^{-}\right) .[10] \mathrm{A}$ neutral state refers to the singly charged dangling bond. The $\mathrm{D}^{\circ}$ is separated from the $\mathrm{D}^{-}$ state by $0.2 \sim 0.3 \mathrm{eV}$ due to the repulsion between two electrons with opposite spins that are

present in the same spatial state.[11,12] The energy associated with this repulsion is called the correlation energy, $U_{c o r}$. For intrinsic a-Si: $\mathrm{H}$, the Fermi energy is located at the center of the $\mathrm{D}^{-}$and $\mathrm{D}^{\circ}$ peaks.

\subsubsection{Substitutional Doping}

According to chemical bonding arguments all the elements in a random amorphous network bond with their optimal valency, irrespective of the neighboring atoms. Chemical bonds are formed such that the maximum number of electrons are paired in bonding 
orbitals, the remaining electrons are paired in non-bonding states and the anti-bonding states are empty. Therefore, an impurity can adapt the local environment to optimize its own bonding configuration. This principle is known as the $8-N$ rule, as the optimum number, $Z$, of covalent bonding for elements is

$$
Z=8-N \text { for } N>4 \text { and } Z=N \text { for } N<4
$$

where $N$ is the number of valence electrons.[13] Accordingly, both boron and phosphorus are expected to be three-fold coordinated and doping will not occur. However, substitutional n-type doping requires that the dopant coordination is such that the one electron is promoted to the anti-bonding state and becomes a free carrier in the conduction band. Therefore, the doping is only a partial deviation from the rule. A positively charged phosphorus atom contains only four valence electrons instead of the usual five, and so its ideal bonding configuration is with a coordination of 4 . The four-fold doping configuration, although of higher energy than the three-fold states, is low enough in energy to be present in reasonable concentrations. In the PECVD process, the addition of diborane $\left(\mathrm{B}_{2} \mathrm{H}_{6}\right)$ and phosphine $\left(\mathrm{PH}_{3}\right)$ to the silane permits the formation of $\mathrm{p}$ doped and $\mathrm{n}$ doped layers.[14]

Consequently, doping efficiency is very low in general. For phosphorus and boron, impurity distribution coefficient, $\delta$, which is the ratio of the solid phase impurity concentration, $C_{s}$, to the molecular concentration in the plasma gas, $C_{g}$, is

$$
\delta_{B, P}=C_{s} / C_{g}=C_{g}-0.2
$$

Active doping efficiency, $\eta$, is defined as the concentration of dopant atoms in the four-fold state divided by the total impurity atom concentration incorporated in the solid phase.

$$
\eta_{B, P}=\left(2 \times 10^{-4}\right) C_{g}^{-0.43}
$$


Total doping efficiency, $\varepsilon_{t o t}$, is the product of $\delta$ and $\eta$, and therefore,

$$
\varepsilon_{\text {tot }}=\left(2 \times 10^{-4}\right) C_{\mathfrak{g}}^{-0.63}
$$

Since there are $5 \times 10^{22}$ atoms per cubic centimeter of amorphous silicon, the active dopant concentration in the a-Si:H film in terms of the gas phase dopant concentration is given by

$$
\left[\mathrm{B}_{4}\right]_{S} \text { or }\left[\mathrm{P}_{4}\right]_{S}=10^{19} \mathrm{C}_{g}^{0.37} \mathrm{~cm}^{-3}
$$

where the subscript 4 represents that the dopant atoms have four-fold configuration.[15]

Doping leads to changes in the density of deep defect levels as well as introducing shallow donor or acceptor levels. Since the $8-N$ rule predicts that doping is not possible in amorphous semiconductors, it has to be extended so as to include charged configurations of the matrix atoms to explain doping in a-Si:H.[16] For example, a positively charged phosphorus atom $\left(\mathrm{P}^{+}\right)$is isoelectronic to a neutral silicon atom $\left(\mathrm{Si}^{\circ}\right)$ and will prefer a fourfold coordination, resulting in a $\mathrm{P}_{4}{ }^{+}$state. Similarly, a negatively charged $\mathrm{Si}$ atom is isoelectonic to a neutral $\mathrm{P}$ atom, thus bonding preferentially as $\mathrm{Si}_{3}^{-}{ }^{-}$[17] This process can be summarized as follows:

$$
\begin{aligned}
& \mathrm{Si}_{4}^{\mathrm{o}}+\mathrm{P}_{3}^{\circ} \longleftrightarrow \rightarrow \mathrm{Si}_{3}^{-}+\mathrm{P}_{4}^{+} \\
& \mathrm{Si}_{4}^{\circ}+\mathrm{B}_{3}^{\circ} \longleftrightarrow \mathrm{Si}_{3}^{+}+\mathrm{B}_{4}^{-}
\end{aligned}
$$

where the superscripts and subscripts represent the charged states and coordination numbers, repectively. Here the three-fold coordinated Si atoms indicate dangling bonds. Therefore, a low energy doping state should consist of ionized dopants and an equal density of dangling bonds.

The variation of the defect density with doping level has been measured by different experiments. For boron and phosphorus, the defect density, $N_{d}$, increases with the square root of the gas phase impurity concentration, and is given by:[18] 


$$
N_{d}=\left(3 \times 10^{19}\right) C_{g}^{1 / 2} \mathrm{~cm}^{-3}
$$

Figure 2.4 shows the variation of the room temperature conductivity with doping in n- and p-type a-Si:H measured by Spear and LeComber.[19] Conductivity, $\sigma_{R T}$, of undoped samples lies in the range around $10^{-9}(\Omega \mathrm{cm})^{-1}$. On the n-side, $\sigma_{R T}$ increases by seven orders of magnitude by phosphine doping. With increasing phosphorus concentration, the Fermi level moves to regions of higher state density which slows down the rate of rise of the conductivity. Increase in the impurity level beyond $10^{-3}$ parts per volume (ppv) does not lead to higher conductivities.

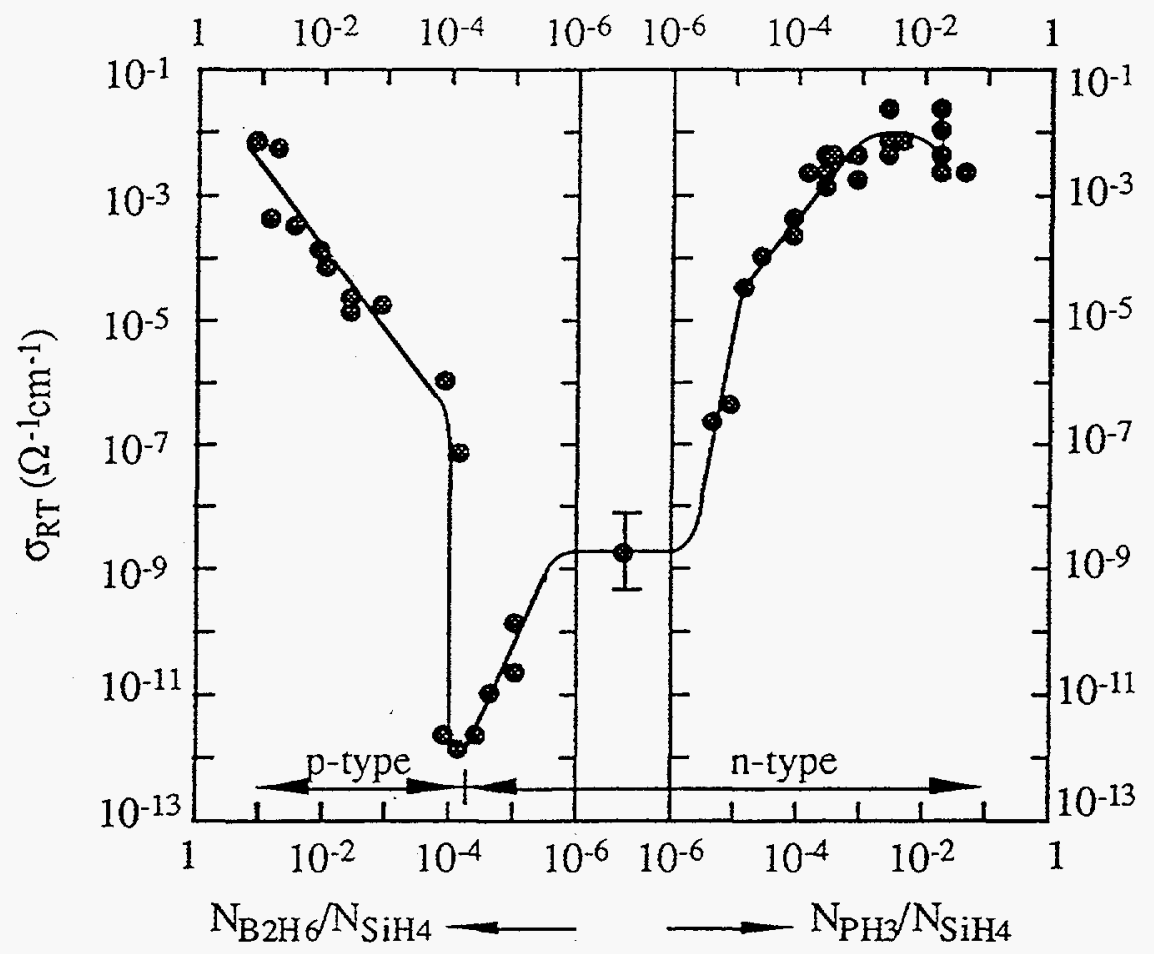

Figure 2.4. The variation of the room temperature conductivity, $\sigma_{R T}$, of aSi:H films doped by the introduction of phosphine and diborane into the deposition gas.[19] 
On the contrary, on the p-side, $\sigma_{R T}$ decreases initially to about $10^{-12}(\Omega \mathrm{cm})^{-1}$. It is because the undoped a-Si:H is slightly n-type as the dangling bonds release their electrons under an electric field. In an undoped specimen, $E_{f}$ lies at about $0.6 \mathrm{eV}$ below $E_{c}$ on the n-side minimum, but hole conduction sets in when $E_{c}-E_{f}$ exceeds $0.85 \mathrm{eV}$.[14] The initial boron doping pulls $E_{f}$ to the p-side of the density of states minimum and the decreasing $\sigma_{R T}$ reflects the rapid reduction in the number of electrons at $E_{c}$. At a diborane concentration of $10^{-4} \mathrm{ppv}$, an almost vertical rise in $\sigma_{R T}$ is observed, indicating that hole conduction has taken over.

\subsubsection{Electronic Transport}

As discussed in section 2.1.2, energy states are localized in the band tail and the tail states affect the carrier transport. Electrons in the conduction bands are easily trapped by the tail states and thermally released in a short time to continue their travel. Therefore, the observed drift mobility, $\mu_{D}$, increases with temperature. At very low temperatures, electron transport is dominated by a hopping mechanism in which electrons jump from one localized state to a neighboring state under the influence of the electric field.

On the other hand, the hole mobility is governed by hopping even at room temperature. Hole transport occurs as a succession of tunnelings through the potential barriers between two adjacent trapping centers.

Generally, the carrier mobility is reduced by scattering, which increases with the degree of disorder, and the energy of the mobility edge within the band also depends on the degree of disorder. Thus, the magnitude of the drift mobility is associated with the widths of band tails, and a comparison of carrier mobilities of samples prepared in different ways may indicate change in the degree of disorder with preparation conditions. Also, because of the asymmetric density of states, with a wider valence band tail than conduction band tail, the average mobility of holes is much lower than that of electrons. The electron and 
hole mobilities can be measured by the Time of Flight (TOF) technique.[20] A schematic diagram of an experimental setup for the time-of-flight technique is illustrated in Fig. 2.5. A p-i-n diode is illuminated with a strongly absorbed light to generate electron-hole pairs at either end according to the sign of the charge carrier to be studied.

Since the mean free path of the light is short, the signal is primarily due to transit of one type of carriers. The injected carriers drift across the sample under a reverse bias, and the transit time $t_{T}$ is measured by an oscilloscope. In this study, an $\mathrm{N}_{2}$ / Dye laser with a duration of 3 nanoseconds (FWHM) is used to produce $510 \mathrm{~nm}$ wavelength light. The drift mobility in terms of the applied bias is given by

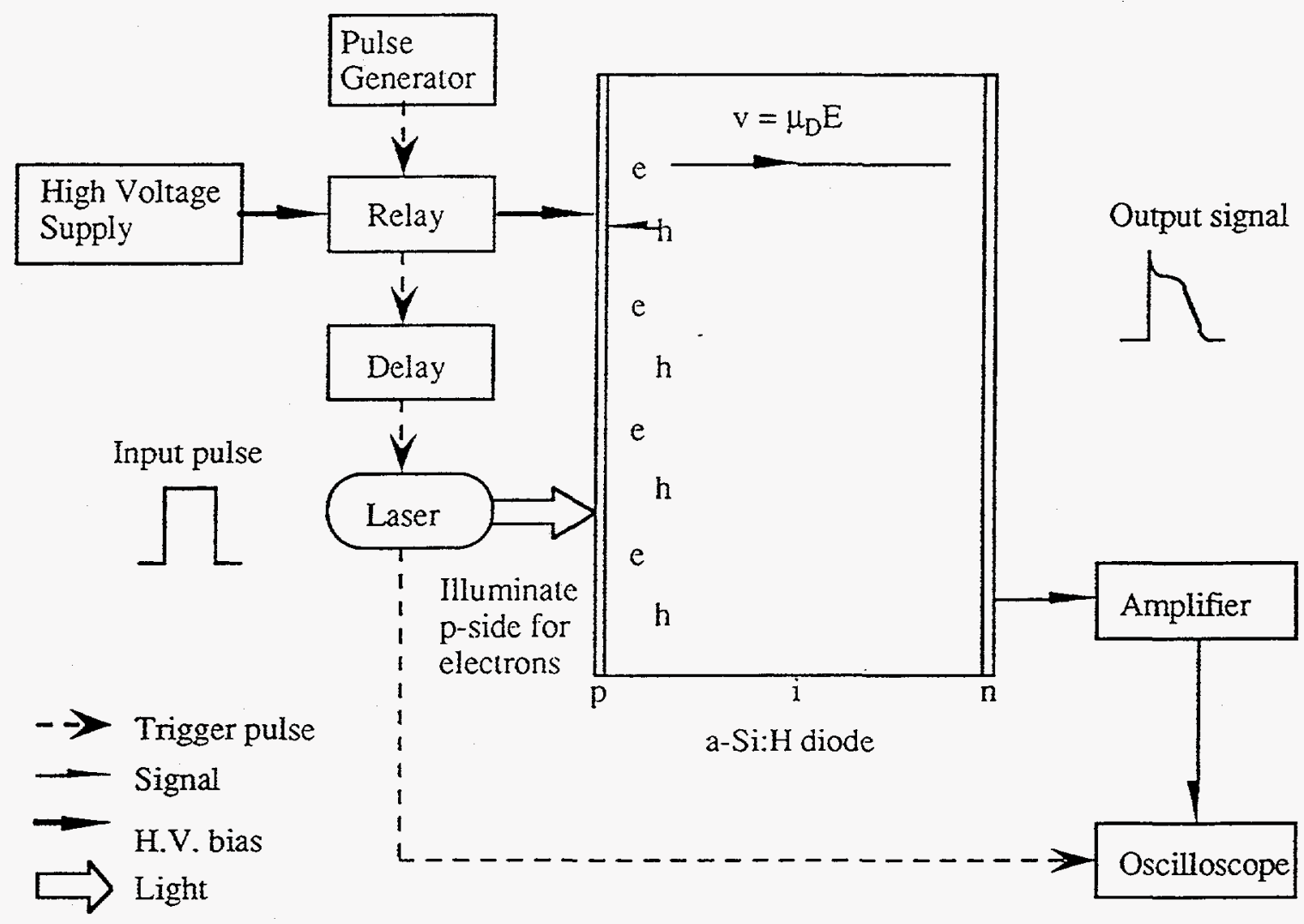

Figure 2.5. Schematic diagram of the time-of-flight apparatus for measuring drift mobility 


$$
\mu_{D}=\frac{v}{E}=\frac{d}{t_{T} E}=\frac{d^{2}}{t_{T} V_{a}}
$$

where $v$ is the carrier velocity, $E$ is the electric field, $d$ is the i-layer thickness, $t_{T}$ is the transit time, and $V_{a}$ is the applied voltage.

The mobility-lifetime product, $\mu \tau$, is a useful parameter in characterizing amorphous semiconductors, since it determines the mean drift length $l_{D}=\mu \tau E$. For $\mu \tau$ measurement, the same experimental setup as time-of-flight is used to measure collected charges, $Q$, as a function of the reverse bias values. The resulting $Q-V$ curve is then fitted to the Hecht equation [21] in order to derive the $\mu \tau$ values.

$$
Q=Q_{o} \mu \tau \frac{V_{a}}{d^{2}}\left[1-\exp \left(-\frac{d^{2}}{\mu \tau V_{a}}\right)\right]
$$

where $Q_{0}$ is the full injected charge.

\subsubsection{Deposition Method}

$\mathrm{A}-\mathrm{Si}: \mathrm{H}$ is deposited most commonly by Plasma Enhanced Chemical Vapor Deposition (PECVD). CVD is defined as the formation of a solid film on a substrate by the reaction of vapor phase chemicals.[22] PECVD uses a radio-frequency-induced glow discharge to transfer energy into the reactant gases, allowing the substrate to remain at a lower temperature. Lower substrate temperature is the major advantage of PECVD, and in fact, PECVD provides a method of depositing films on substrates that do not have the thermal stability to accept coating by other methods. A schematic diagram of a typical PECVD apparatus is shown in Fig. 2.6. 


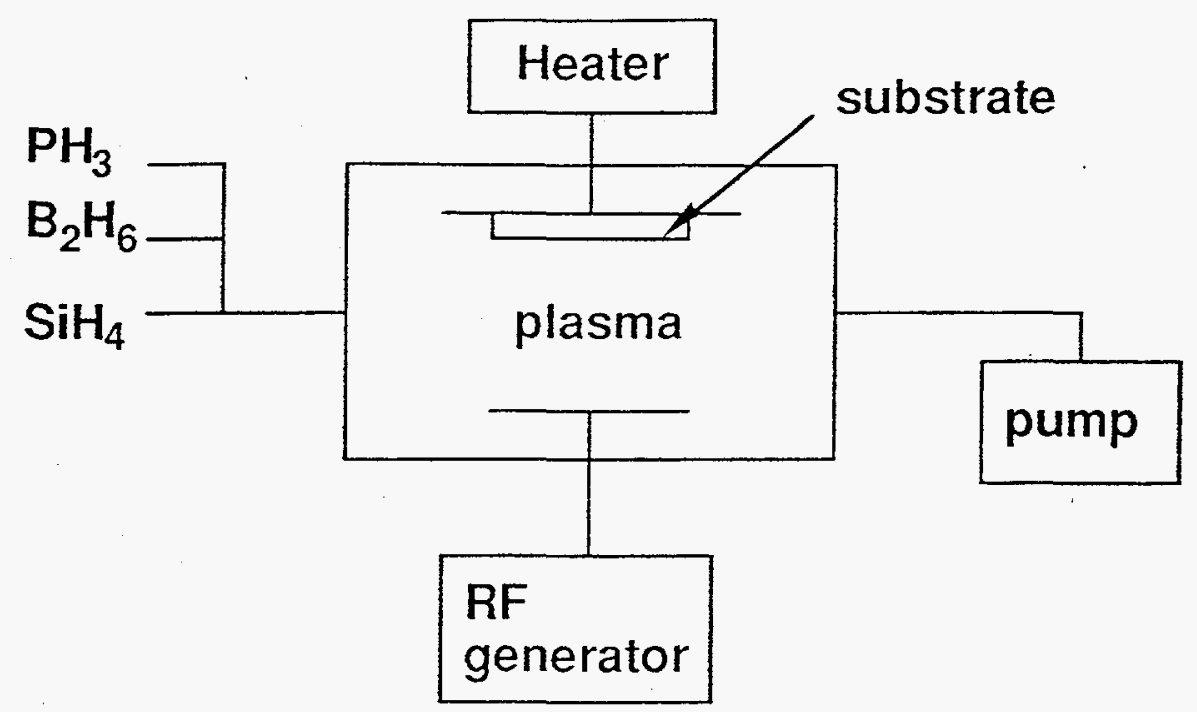

Figure 2.6. Schematic diagram of a PECVD system

Although the principle of the deposition is quite simple, the physical and chemical processes which take place are extremely complex, and it is difficult to identify the dominant reaction paths. Several researchers have suggested models for the growth kinetics of a-Si:H film from $\mathrm{SiH}_{4}$ plasmas using mass spectroscopy and optical emission spectroscopic techniques.[23, 24] On the basis of these data the hydrogen elimination process from the film and the polyhydride formation can be explained. According to this model the $\mathrm{SiH}_{2}$ free radicals react with the $\mathrm{Si}-\mathrm{H}$ bond on the growing surface and produce an activated complex that can eliminate $\mathrm{H}_{2}$ molecules. A simplified reaction scheme has been proposed as follows:

i) gas phase ionization

$$
\begin{aligned}
& \mathrm{SiH}_{4} \rightarrow \mathrm{SiH}_{\mathrm{n}}^{+}+(4-\mathrm{n}) \mathrm{H}+\mathrm{e}^{-} \quad \mathrm{n}=1,2,3 \\
& \mathrm{H} \rightarrow \mathrm{H}^{+}+\mathrm{e}^{-}
\end{aligned}
$$

ii) gas phase dissociation

$$
\begin{aligned}
& \mathrm{SiH}_{\mathrm{n}} \longrightarrow \mathrm{SiH}_{\mathrm{n}^{\prime}}+\left(4-\mathrm{n}^{\prime}\right) \mathrm{H} \quad \mathrm{n}, \mathrm{n}^{\prime}=1,2,3 \\
& \mathrm{H}_{2} \longrightarrow 2 \mathrm{H}
\end{aligned}
$$


iii) insertion of an $\mathrm{SiH}_{2}$ radical into an $\mathrm{Si}-\mathrm{H}$ surface bond

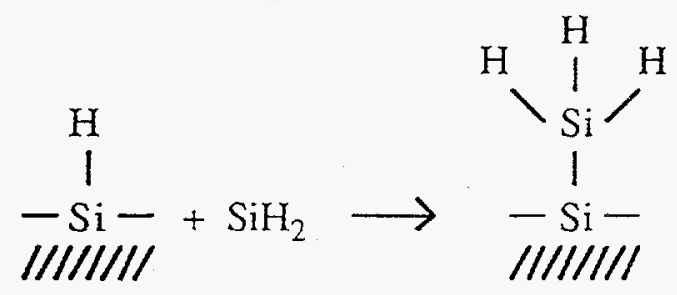

iv) hydrogen elimination

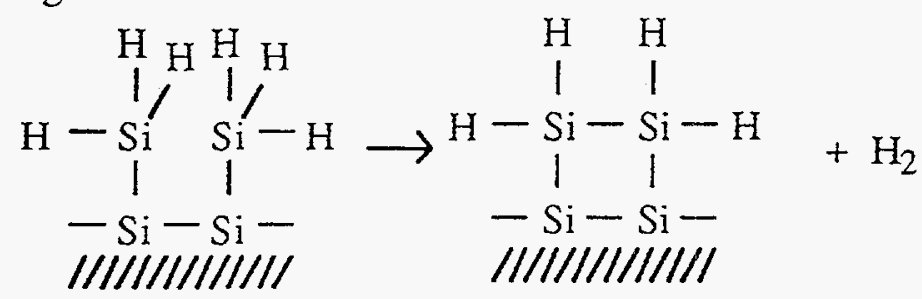

The amount of hydrogen remaining in the film is determined by a competition between $\mathrm{H}_{2}$ elimination and deactivation of the complex. The films produced at low temperature generally have more $\mathrm{H}$ concentration, leading to formation of polymeric type structures with a greater number of $\mathrm{Si}-\mathrm{H}_{2}$ and $\mathrm{Si}-\mathrm{H}_{3}$ groups. It is the fundamental change from polymeric type structure for films deposited at a low temperature to monohydride type at a higher temperature that has a major effect of the reduction of the density of states in the band gap.

All the samples used in this study were prepared by the GSI PECVD system at Lawrence Berkeley Laboratory. An optimum deposition condition to produce good quality materials has been determined to be: a silane flow rate of $40 \mathrm{sccm}$, chamber pressure of 300 mTorr, $R F$ power density of $40 \mathrm{~mW} / \mathrm{cm}^{2}$, a substrate temperature of $250^{\circ} \mathrm{C}$, and an $R F$ frequency of $85 \mathrm{MHz}$ for intrinsic layers. These parameters are referred to as "standard conditions" throughout this dissertation. Under these conditions, a growth rate of 2.3 $\mu \mathrm{m} /$ hour and an ionized dangling bond density of $5 \sim 7 \times 10^{14} \mathrm{~cm}^{-3}$ were achieved. 


\subsection{RAdiation Detection SCHEMES}

\subsubsection{Radiation Detection Principles}

The operation of a radiation detector depends on the manner in which the radiation interacts with the material of the detector itself.[25] Charged particle radiations, such as alpha particle and fast electrons, continuously interact primarily through the coulomb force with the electrons present in the medium. Upon entering any absorbing medium, the charged particle interacts simultaneously with many electrons. In any encounter, the electron feels an impulse from the coulomb force as the particle passes its vicinity. This impulse may be sufficient either to raise the electron to a higher energy level within the absorber atom (excitation) or to knock it out of the atom (ionization). Since the maximum energy that can be transferred from a charged particle to an electron in a single collision is only a small fraction of the total energy, the primary particle must lose its energy in many such interactions during its passage through an absorber in order to be detected. At any given time, the particle is interacting with many electrons, so the net effect is to decrease its velocity continuously until the particle is stopped. The rate of energy loss is called the linear stopping power and is defined as the differential energy loss for a charged particle within a material divided by the corresponding differential path length.

Uncharged radiation, such as neutron, $X$-ray and $\gamma$-rays, first undergoes an interaction with the nucleus or electron shells of constituent atoms. The interaction mechanisms include photoelectric absorption, Compton scattering, and pair production. The incident radiation transfers all or part of its energy to electrons or nuclei of the constituent atoms or to charged particle products of nuclear reactions. These secondary electron or photon "signals" are collected and processed by an external circuit of the radiation detector. 
Radiation detectors can be categorized as follows according to the type of interacting medium. Choice of a certain type of detector depends on the specific radiation types an detection condition.

(1) Gas-filled detectors: The majority of gas-filled detectors are based on collecting the charged created by direct ionization or by a secondary process within the gas through the application of an electric field. Examples are ionization chambers, proportional counters, and Geiger-Müller tubes.

(2) Scintillation counters: Plates or crystals of scintillators convert the incident radiation into visible light and photomultiplier tubes or semiconductor photodiodes detect the photons. The scintillation process is one of the oldest and most useful methods for the detection and spectroscopy of a wide assortment of radiations.

(3) Semiconductor detectors: When an incident charged particle passes through a semiconductor, electron hole pairs are produced along the track of the particle. The ionization energy, the average energy expended to produce one electron hole-pair, is known to be largely independent of both the energy and type of the incident radiation and much smaller than that in typical gas-filled detectors or scintillation counters. Semiconductor detectors as a solid state detector also have advantages over the gas-filled detectors since the detector dimensions can be kept smaller. Scintillation detectors use crystalline, solid or liquid medium; they have relatively poor energy resolution compared to the semiconductors. 


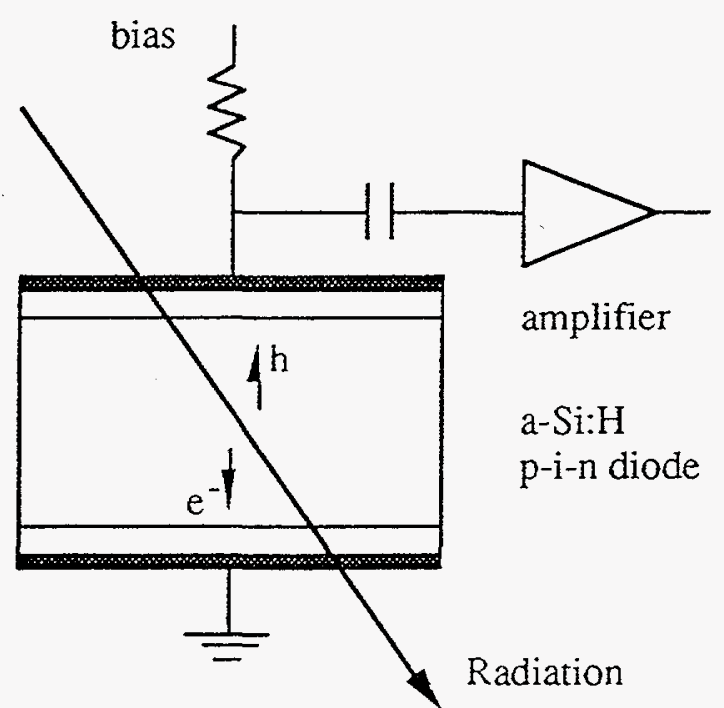

(a)

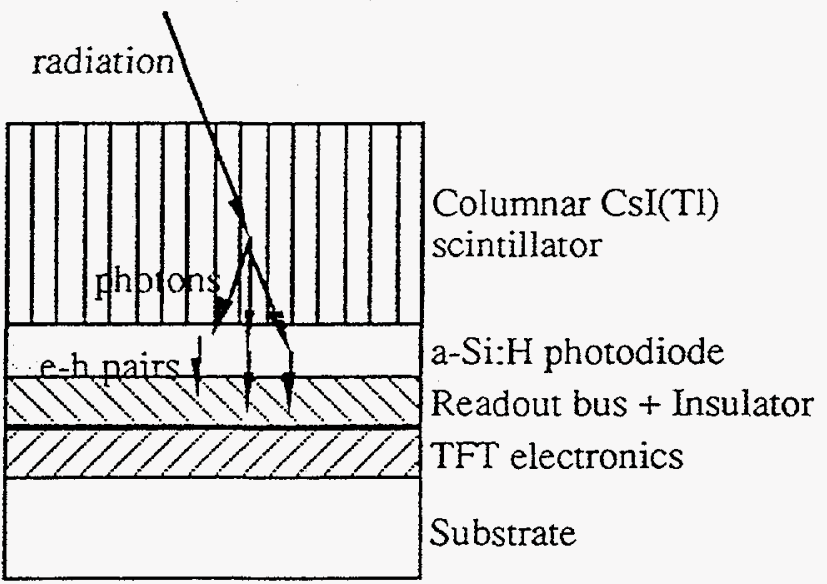

(b)

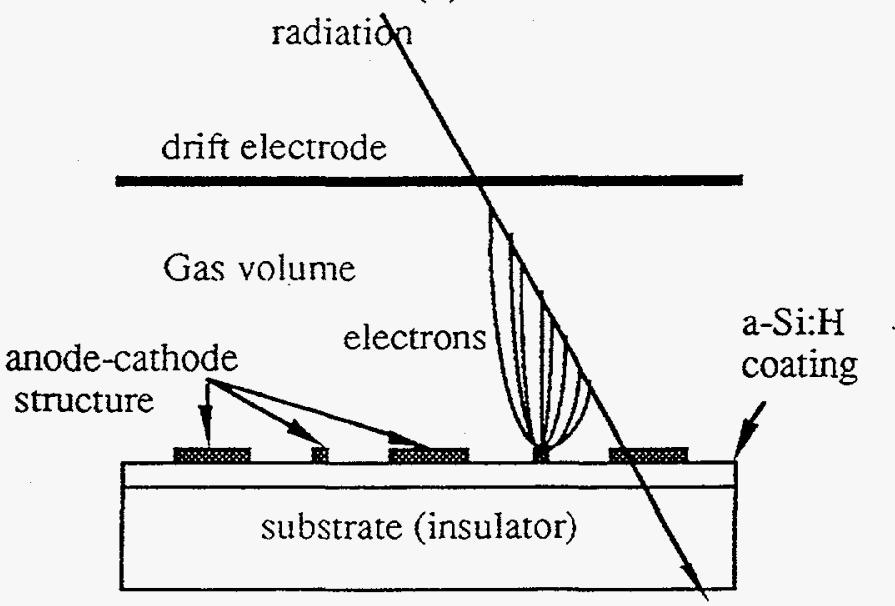

(c)

Figure 2.7. Detection schemes of a-Si:H based radiation detectors. (a) direct detection (b) indirect detection by coupling with a scintillator (c) gasfilled detector 


\subsubsection{Amorphous Silicon Detectors}

Amorphous silicon detectors have several advantages over crystalline counterparts despite its inferior electronic properties.[26] Firstly it exhibits high radiation resistance. It has been demonstrated that the a-Si:H diodes are appreciably radiation resistant to $1 \mathrm{MeV}$ neutron fluences in excess of $4 \times 10^{14} \mathrm{~cm}^{-2}$ whereas the crystalline diodes failed at $10^{13}$ $\mathrm{cm}^{-2}$ fluence. $[27,28]$ Further, large areas can be fabricated, by a low cost process, in thin film form, while the area of crystalline detectors is limited by the wafer size. In addition, conduction band electrons created by ionization need to travel a shorter distance ( $1 \sim 50$ $\mu \mathrm{m})$ before collection, compared to crystalline detectors $(400 \sim 700 \mu \mathrm{m})$, and this to some extent compensates the longer collection time arising from the low mobility of these carriers.

A-Si:H can be used in all three types of detectors discussed in the previous section. Figure 2.7 illustrates some possible detection schemes for a-Si:H radiation detectors. In the direct detection scheme as shown in Fig 2.7(a), a thick, fully-depleted intrinsic layer is required. Details of the fully depleted detectors will be discussed in section 3.1.1. The radiation-induced electron-hole pairs drift in the electric field. The induced current is subsequently amplified in an external circuit. If ohmic contacts are used on opposite sides of the intrinsic layer, the leakage current is too high and the radiation induced current is buried in the fluctuation of the background current. Instead, p- and n-type materials are used as blocking contacts so that the charge carriers initially removed by the application of an electric field are not replaced at the opposite electrode and the leakage current can thus be reduced to a sufficiently low value.

In the indirect detection scheme as shown in Fig. 2.7(b), a scintillator converts the incident radiation into visible light. A scintillator such as CsI crystal doped with TI produce light in the wavelength of $350 \sim 600 \mathrm{~nm}$ range. The resulting photons can be absorbed in a 
thin $(1 \sim 2 \mu \mathrm{m})$ a-Si:H p-i-n photodiode and produce electrons and holes which are drifted by the applied bias.

In the microstrip configuration as shown in Fig. $2.7(\mathrm{c})$, a very thin $(<1 \mu \mathrm{m})$ layer of a-Si:H is coated on the alternating anode-cathode structure to control the surface resistivity of the microstrip chamber. The electrons produced in the primary ionization process by the incident radiation are drifted towards the anode and are multiplied in the avalanche process due to the intense electric field created by the geometry of the anodecathode array. Positive ions tend to pile-up around the anode due to their low mobility and decrease the electric field concentration at the anode. The a-Si:H coating provides mobility to the positive ions which land on the surface of the substrate so that they can be swept out to the cathode. Surface resistivity of the coated substrate must be kept between $10^{12}$ and $10^{16} \Omega / \square$ to suppress the leakage current which increases noise. The a-Si:H layer is doped or alloyed with other elements to provide suitable resistivity and other electrical properties. 


\subsection{RESIDUAL STRESS IN THIN FILMS}

Mechanical stability and strong adhesion is one of the major concerns in thin film electronic devices. The mechanical properties of thin films are different from those of bulk materials because of their unique microstructure, large surface-to-volume ratio, reduced dimensions, and the constraints caused by the substrate.[29] In most cases, large internal stresses of the order of mega- or even giga-pascal develop in thin films during their growth. A stress of this magnitude is comparable to the yield strength of most bulk metals and also sufficient to overcome the adhesion between the film and substrate and to result in peeling of the film.[30]

\subsubsection{Origin of the Stress}

The residual stress developed during preparation of thin films consists of thermal $\left(\sigma_{t h}\right)$ and intrinsic $\left(\sigma_{i}\right)$ components. Both components are basically due to the constraint imposed by the film-substrate bonding. The thermal stress arises from the mismatch of the thermal expansion coefficients between the film and the substrate during cooling down from the deposition temperature to room temperature. The thermal component is given by

$$
\sigma_{t h}=\left(\alpha_{f}-\alpha_{s}\right)\left[E_{f} /\left(1-v_{f}\right)\right]\left(T_{d}-T_{m}\right)
$$

where $\alpha_{f}$ and $\alpha_{s}$ are the average coefficients of expansion for film and substrate, respectively, $T_{d}$ and $T_{m}$ are the temperatures of the substrate during film deposition and at measurement, respectively, and $E_{f}$ and $v_{f}$ are the Young's modulus and Poisson's ratio of the film, respectively.

The contribution of intrinsic, or growth stress to the total stress reflects film structure in a way not well understood at present. The origin of intrinsic stresses has been 
reviewed extensively by many authors.[31-35] The proposed models can be categorized as follows:

(1) Surface Energy: Deposition of thin films produces an additional interface. Due to the increased interfacial area, substrate bending occurs in order to minimize the total energy of the system which is the sum of surface energy and strain energy.

(2) Coalescence of Crystallites or Clusters: Densification associated with coalescence of isolated clusters leads to tensile stress on the film. It includes grain growth and the filling and closure of channels and holes.

(3) Nonequilibrium Growth: If material is deposited in a metastable state, subsequent atomic rearrangement, which would result in a shrinkage of the film if it were not attached to the substrate, generates stress. Examples are crystallization, vacancy annihilation to grain boundaries, grain boundary relaxation, shrinkage of grain boundary voids.

(4) Impurity effects: Incorporation of impurity atoms, for example, residual gases or chemical reactions changes the film stress. Especially, gas molecules present in the atmosphere during deposition contribute to the film stress by diffusing into the film after growth or by affecting the nucleation and growth condition.

(5) Phase Transformation and Precipitation: Volume changes associated with phase transformation and particle precipitation also lead to stress generation in thin films.

(6) Epitaxy: Strain can be introduced into a growing film if the film grows with an epitaxial relationship to the substrate and a mismatch between the lattice spacings of the two materials exist. As the thickness of the epitaxial layer increases, the strain energy in the film increases until it becomes more energetically favorable for a series of misfit dislocations to form at the interface.

Among the models mentioned above, (2) and (6) are ruled out because they only apply to crystalline films. (5) is also not taken into account because no phase transition is involved in the growth of amorphous silicon films. Origin of the stress observed in a-Si:H film will be discussed in more detail in the next chapter. 


\subsubsection{Mechanics and Measurement of Stress in Thin Films on Substrates}

Figure 2.8 shows schematic diagrams illustrating the development of films stresses on substrates. It is assumed that the lateral dimension of the film is much greater than the thickness and that the substrate is much thicker than the film. We can start by considering a free-standing film which is free of stress.(Fig. 2.8(a)) If the film experiences a uniform expansion with a dilatation strain, $e^{T}$, in the detached strain (Fig. 2.8(b)), the principal strain components are:

film

(a)

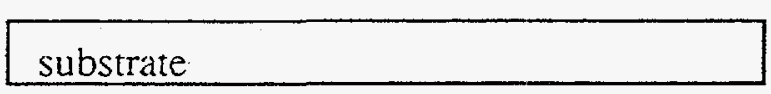

(b)

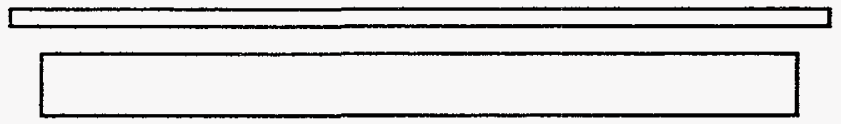

(c)

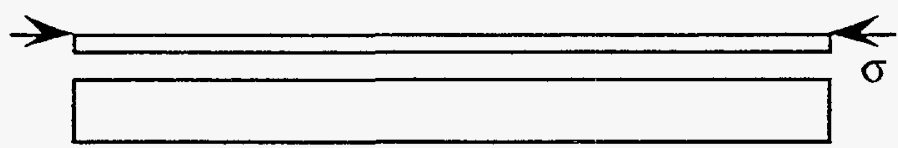

(d)

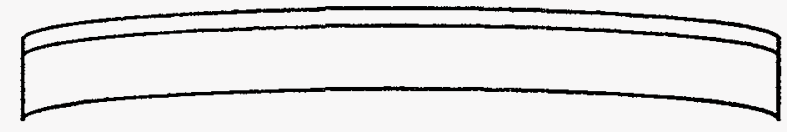

(e)

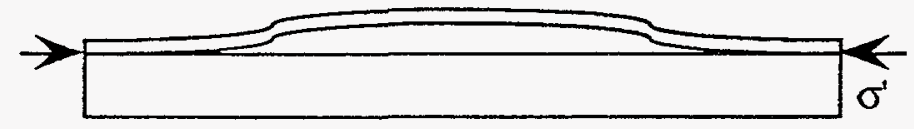

Figure 2.8. Illustration of the development of film stresses and the relationship between the biaxial stress and the associated substrate bending or delamination.[35] 


$$
e_{x x}=e_{y y}=e_{z z}=e^{T} / 3
$$

where the first subscripts represent the direction of the force acting on the film and the second subscripts direction normal to the plane on which the force exerts. To reattach the film to the substrate, because the lateral dimensions of the film no longer match those of the substrate, a biaxial stress must be imposed on the film to deform it elastically so that it again fits the dimensions of the substrate.(Fig. 2.8(c)) The stress required to do this produces elastic strains, $e_{x x}$ and $e_{y y}$, that compensate exactly these components of the transformation strain.

$$
e=e_{x x}=e_{y y}=-e^{T} / 3
$$

Using Hooke's law, this leads to a biaxial stress in the film:

$$
\sigma=\sigma_{x x}=\sigma_{y y}=e E /(1-v)
$$

As long as the compressive forces to deform the film are present, the stress in the film does not change when it is reattached to the substrate. When these forces are removed by superimposing forces of opposite signs, these additional forces remove the normal tractions from the edges of the film, and they produce shear stresses on the film / substrate interface near the edges of the film. These shear stresses provide the forces needed to maintain the biaxial stress in the film and also cause the substrate to bend elastically.

If adhesion between the film and substrate is not strong enough, delamination process occurs by interface cracking and its propagation.(Fig. 2.8(e)) The resultant stress, $\sigma^{\prime}$ is smaller than the prestress $\sigma$. This is another mechanism of strain energy release, which is stored in the film and substrate. This process will be discussed further in the next section. 
The amount of bending depends on the thickness and biaxial elastic modulus, $E_{s} /\left(1-v_{s}\right)$, of the substrate. A simple biaxial bending analysis shows that the radius of curvature is given by

$$
\frac{1}{R}=\frac{1-v_{s}}{E_{s}} \cdot \frac{6 \sigma t_{f}}{t_{s}^{2}}
$$

where $\sigma$ is the biaxial compressive stress in the film, $t_{f}$ and $t_{s}$ are thicknesses of the film and substrate, respectively.[36] Rearranging this equation gives the total stress in the film as a function of the substrate curvature caused by that stress.

$$
\sigma_{t o t}=\frac{E_{s}}{6\left(1-v_{s}\right)} \frac{t_{s}^{2}}{t_{f}} \frac{1}{R}
$$

Therefore, most of the stress estimation methods are based on the measurement of the substrate curvature and can be categorized as a contact and a non-contact method. The contact system uses a mechanical stylus to scan the backside of a substrate. Alphastep 200 Profilometer was used in this study. The radius of curvature is calculated from the profile:

$$
\frac{1}{R}=\frac{2 Z}{L^{2}+Z^{2}}
$$

where $L$ is the scanned distance and $Z$ is the deflection of the center of the scan relative to both ends. The non-contact system uses laser light or ultrasound to scan the substrate. The change in angle of the reflected beam or change in distance between the ultrasonic head and the substrate surface according to the surface profile provide information on the substrate curvature.

For the case of single crystal substrates, the curvature of the substrate produces a distortion of the crystal lattice that can be detected by X-ray diffraction or photoluminescence spectra. In such a case it is not necessary to measure the curvature 
before the film is deposited or after the film is removed from the substrate, because the latrice constant for most good substrates, such as silicon wafers, is well known.

\subsubsection{Delamination and Spallation}

The binding of the film atoms to the substrate has been called adhesion. In the process of film formation, film atoms arrive at the substrate randomly and move to find suitable minimum energy positions. However, because of the substrate restriction and the very rapid thermal energy dissipation, the atom migration is impeded and part of the energy carried by the atoms remains in the films as strain energy. Therefore, the generation of internal stress should be closely connected with the adhesion.[37] When the value of the strain energy is large enough to overcome the adhesion energy, spontaneous peeling of the film from the substrate occurs.

Figure 2.9 illustrates the delamination mechanisms of the films in residual stress. When the film is in residual tension, delamination originates preferentially at the specimen edges and propagates inward. The film has a tensile force with respect to the substrate that creates a net momentum at the edge of the film.[38] This is called "edge effect", and, when the net momentum at the edge is greater than the adhesion forces holding the film to the substrate, the film pulls upward and off. For the other case of the films in compression, delamination involves buckling followed by spallation. Buckling usually starts at the initial interface separation or cracks extending along a plane parallel to the surface. Spalling involves the subsequent propagation of the crack through the film.

Interface delamination in the absence of buckling does not cause redistribution of stress or energy release. However, when the delaminated region buckles away from the substrate, there is a release in energy with crack advance which can be determined from the difference between the elastic strain energy stored in the buckled and unbuckled configurations.[39] 


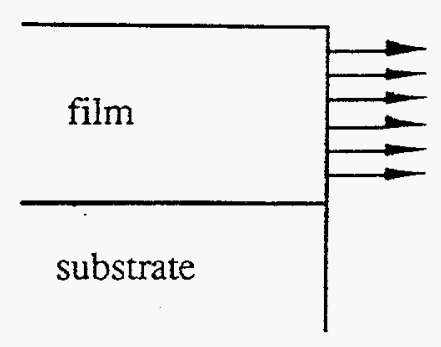

tension in film

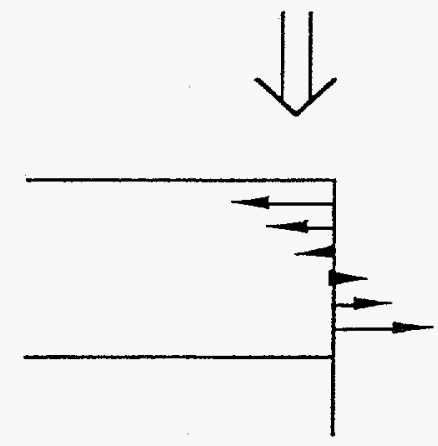

net momentum

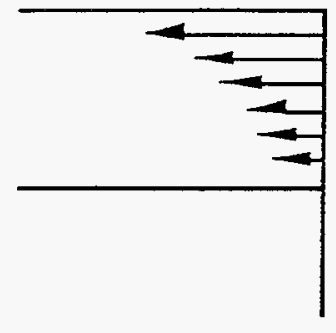

edge effect

(a)
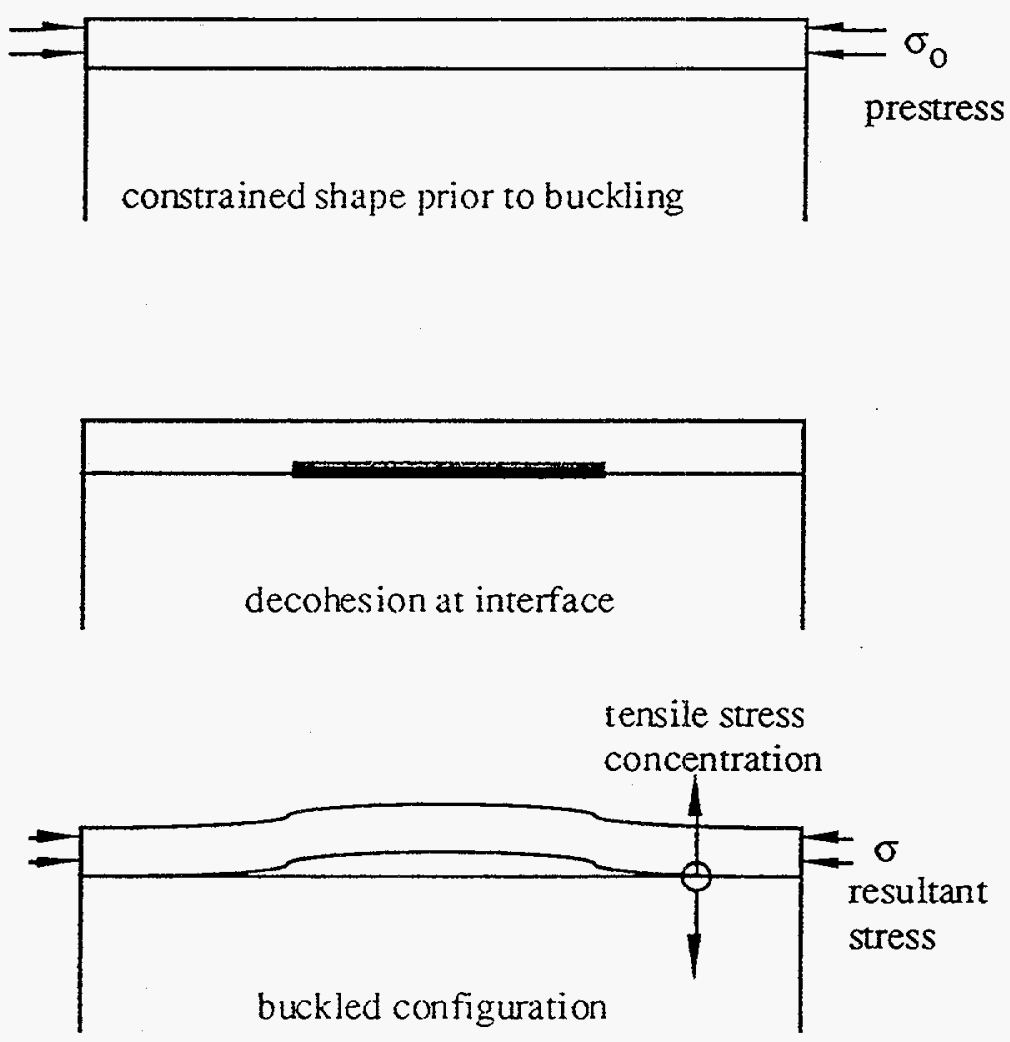

(b)

Figure 2.9 Schematic illustration of delamination mechanisms. (a) a film under tension (b) a film under compression. 


\section{References}

[1] R.A.Street, "Hydrogenated Amorphous Silicon", Cambridge Univ. Press (1991)

[2] W.Schulke, Phil. Mag. B 43451 (1981)

[3] F.A.Sarott, Z.Iqbal and S.Veprek, Sol. State. Commun., 42-6 465 (1982)

[4] S.Qureshi, "Hydrogenated Amorphous Silicon Radiation Detectors: Material Parameters; Radiation Hardness; Charge Collection", Ph.D. Thesis, U.C. Berkeley, (1991)

[5] M.H.Brodsky, M.A.Frisch, J.F.Ziegler and W.A.Lanford, Appl. Phys. Lett., 30 $561(1977)$

[6] L.Ley, "Photoemission and Optical Properties," Chap. 3. in The Physics of Hydrogenated Amorphous Silicon II, ed. by J.D.Joannopoulos and G.Lucovsky, pp. 142, Springer Verlag, Berlin (1984)

[7] J.Tauc, R.Grigorovich and A.Vancu, Phys. Stat. Sol., 15627 (1966)

[8] P.W.Anderson, Phys. Rev., 1091492 (1958)

[9] N.F.Mott and E.A.Davis, "Electronic Processes in Non-Crystalline Materials", Oxford Univ. Press, Oxford (1979)

[10] G.Cho, "Signal and Noise Analysis of a-Si:H Radiation Detector-Amplifier System", Ph.D. Thesis, U.C. Berkeley (1992)

[11] P.W.Anderson, Phys. Rev. Lett., 34953 (1975)

[12] R.A.Street and N.F.Mott, Phys. Rev. Lett., 351293 (1975)

[13] N.F.Mott, Adv. Phys., 1649 (1967)

[14] W.E.Spear and P.G.LeComber, Solid State Commun., 17, 1193 (1975)

[15] M.Stutzmann, Phil. Mag. B 355666 (1987)

[16] R.A.Street, Phys. Rev. Lett., 491187 (1982)

[17] M.Stutzmann, D.K.Biegelsen and R.A.Street, Phys. Rev. B 355666 (1985) 
[18] R.A.Street, D.K.Biegelsen, W.B.Jackson, N.M.Johnson and M.Stutzmann, Phil. Mag. B 52235 (1985)

[19] W.E.Spear and P.G.LeComber, Phil. Mag., 33 6 935 (1976)

[20] W.E.Spear, J. Non-Cryst. Sol., 1197 (1968)

[21] R.A.Street, Phys. Rev. B 274924 (1983)

[22] S.Wolf and R.N.Tauber, "Silicon Processing for the VLSI Era" Vol.1, Lattice (1986)

[23] F.J.Kampas and R.W.Griffith, Appl. Phys. Lett., 39407 (1981)

[24] W.A.P.Claassen, Thin Solid Films, 16889 (1988)

[25] G.F.Knoll, "Radiation Detection and Measurement", 2nd Ed., John Wiley \& Sons, New York (1979)

[26] J.S.Drewery, G.Cho, I.Fujieda, T.Jing, S.N.Kaplan, V.Perez-Mendez, D.Wildermuth and R.A.Street, Nucl. Instr. Meth., A 310165 (1987)

[27] V.Perez-Mendez, S.N.Kaplan, W.Ward, S.Qureshi and R.A.Street, Nucl. Instr. Meth., A 260195 (1987)

[28] V.Perez-Mendez, S.N.Kaplan, G.Cho, I.Fujieda, S.Qureshi, W.Ward and R.A.Street, Nucl. Instr. Meth., A 273127 (1988)

[29] R.W.Hoffman, "Mechanical Properties of Non-Metallic Thin Films", in NATO Adv. Study Institute Series B, Vol. 14, Eds. by C.H.S.Dupuy and A.Cachard, Plenum Press, New York, pp. 273 (1976)

[30] K.L.Chopra, Thin Film Phenomena, R.E.Krieger Pub. Co., Huntington (1969)

[31] K.Kinosita, Thin Solid Films, 1217 (1972).

[32] R.W.Hoffman, Phys. Thin Films, 3211 (1966)

[33] D.S.Cambell, "Handbook of Thin Film Technology", Eds. by L.I.Maissel and R.Glang, pp.123, McGraw-Hill, New York, (1970)

[34] D.S.Cambell, "Basic Problems in Thin Film Physics", Eds. by R.Niedermayer and H.Mayer, pp.223 Vandenhoeck and Ruprecht, Gottingen (1966) 
[35] M.F.Doerner and W.D.Nix, "Stresses and Deformation Processes in Thin Films on Substrate", in CRC Critical Reviews in Solid State and Materials Sciences, $14 \underline{3}$ pp.225 (1988)

[36] A.Brenner and S.Senderoff, J. Res. Nat'l Bureau of Standards, 42105 (1949)

[37] A.Kinbara, S.Baba and N.Matuda, Thin Solid Films, 141229 (1986)

[38] N.Naito, "Deposition of Diamond on Boron-Doped Si and SiC", Ph.D. Thesis, U.C.Berkeley, (1992)

[39] A.G.Evans and J.W.Hutchinson, J. Solid Str., 20455 (1984) 


\section{CHAPTER 3. THICK $(\sim 50 \mu \mathrm{m})$ a-Si:H LAYERS FOR DIRECT DETECTION OF MINIMUM IONIZING PARTICLES}

\subsection{INTRODUCTION}

In high energy physics experiments, detection of charged particles is of interest. In order to detect charged particles, especially minimum ionizing particles, p-i-n diodes with a thick $(>50 \mu \mathrm{m})$ intrinsic layer are required in order to produce a sufficient number of electron-hole pairs in the intrinsic layer. Figure 3.1 shows a typical radiation detection system. Electron-hole pairs which are created within the i-layer by the passage of radiation will be swept out of the i-layer by the electric field, and their motion constitutes an electrical signal. The signal is processed as a voltage pulse in the following electronic system and the information is represented as a pulse height.

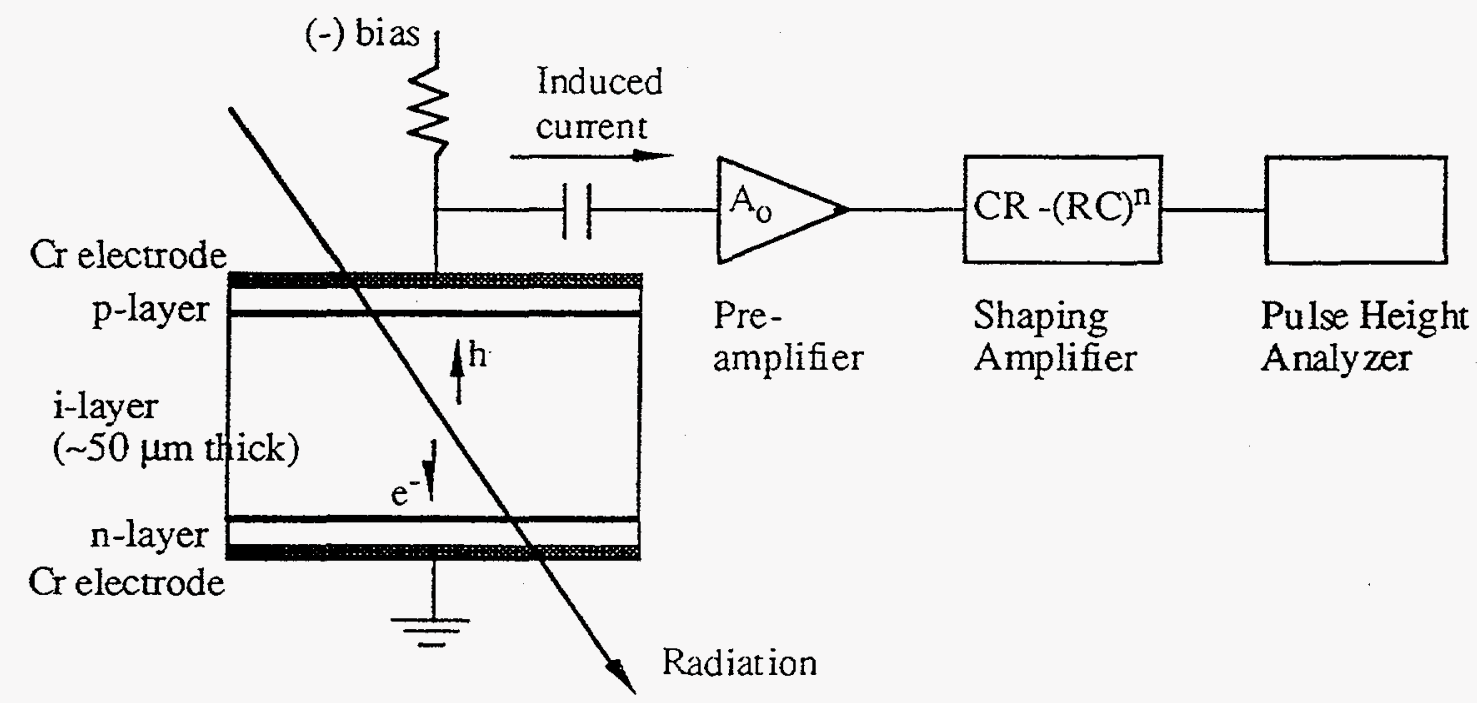

Figure 3.1. Schematic diagram of a thick a-Si:H detector and signal processing electronics for direct detection of minimum ionizing particles 
However, preparation and operation of such thick a-Si:H diodes has been difficult because of high dangling bond density, low deposition rate, and high residual stress. The dangling bonds modify the electric field inside the intrinsic layer and reduce the mean free path of charge carriers by trapping, making it necessary to apply high electric fields (10 $50 \mathrm{~V} / \mu \mathrm{m})$. Low deposition rate limits the throughput of device production and raises the fabrication cost. High built-in stress bends the substrate or causes the film to peel off spontaneously. In the following sections, these problems will be addressed in detail, and the deposition methods to overcome such difficulties will be discussed.

\subsubsection{Fully-Depleted Detectors}

Once electrons and holes are formed in a semiconductor, they tend to migrate either by diffusion or under the influence of an applied electric field until they are either collected at an electrode or recombined. As carrier diffusion plays little role in the a-Si:H due to a low diffusion coefficient, the presence of a sizable electric field is essential for charge collection.

As mentioned in section 2.1.3, the undoped a-Si:H used to form the i-layer is slightly n-type because the electrons are released from the defect states. For an unbiased diode, the built-in potential which is formed spontaneously across the diode is inadequate to generate a large enough electric field to make the charge carriers move very quickly. Therefore, charges can be lost due to trapping and recombination, and charge collection will be incomplete. The external reverse bias voltage causes any electrons created in or near the junction to be swept back toward the n-type material and any holes toward the ptype side. The intrinsic region is thus "depleted" in that the concentration of holes and electrons is greatly suppressed. The width of the depletion region represents the active volume of the detector and is changed by varying the reverse bias. Charge carriers created 
within the depletion region of the reverse biased detectors can be quickly and efficiently collected.

As the reverse bias voltage is raised from zero, the electric field extends further from the p-side into the bulk of the $\mathrm{i}$-layer. For low values of the voltage, the $\mathrm{i}$-layer is only partially depleted and the electric field goes to zero at the far edge of the depletion region. Between this point and the $\mathrm{n}$-side, a region of undepleted silicon exists in which there is no electric field. This region then represents a dead layer from which charge carriers are not collected.

If the applied voltage is increased further, the depletion region may extend all the way to the $\mathrm{i}-\mathrm{n}$ interface. At this stage, a finite electric field exists all the way through the $\mathrm{i}$ layer, and the back dead layer thickness is reduced to that of the $\mathrm{n}$ blocking contact. Many semiconductor detectors are operated with sufficient reverse bias voltage so that the depletion region extends through the entire device thickness except the blocking layers, creating a fully depleted detector. Because of the several advantages of this configuration, the fully depleted detector is preferred in a number of applications.[1]

Fully depleted detectors are useful as transmission detectors for particles having sufficient energy to pass completely through the wafer. The pulse amplitude indicates the energy lost by the incident radiation during its transit through the device. Good energy resolution depends on achieving a small detector capacitance and is thus promoted by using a voltage with which the detector becomes fully depleted. Also, the finite electrical resistance of the dead layer in partially depleted detectors is a source of Johnson noise which is associated with series resistance.[2] It is eliminated by extending the depletion region all the way to the back contact.

Timing properties are superior in fully depleted configurations because carrier velocities become very low at the edge of the depletion region in a partially depleted detector. In a fully depleted detector, the electric field can be maintained at a high value everywhere within the detector volume. 


\subsubsection{Minimum Ionizing Particles}

The stopping power, $d E / d x$, of a particle going through some medium is given by Bethe formula[3]:

$$
-\frac{d E}{d x}=\frac{4 \pi Z^{2} e^{4}}{m v^{2}} n_{e}\left[\ln \left(\frac{2 m v^{2}}{I\left(1-\beta^{2}\right)}\right)-\beta^{2}\right]
$$

where $Z$ is atomic number of the particle, $v$ is particle velocity, $m$ is electron mass, $n_{e}$ is

electron density in the medium, $I$ is an average ionization potential, $\beta$ is $\nu$ divided by the speed of light, and $e$ is the electron charge.

At medium energies the stopping power decreases inversely with particle energy. A broad minimum in stopping power is seen at $1.5 \sim 3$ times the rest mass of the particle, and the particles having this range of energy are called minimum ionizing. As discussed in section 2.2.1, minimum ionizing particles (MIPs) deposit only a small fraction of their total energy when passing through a detector and produce a small number of electron-hole pairs. Hence, detection of minimum ionizing particles above noise requires an a-Si:H layer of thicknesses of tens of microns which could be depleted at a reasonable bias.

The average number of electron hole pairs, $n_{\min }$, created from the interaction of the incident minimum ionizing radiation in intrinsic a- $\mathrm{Si}: \mathrm{H}$ layer is given by

$$
n_{\min }=\frac{(\Delta E)_{\min }}{W}
$$

where $W$ is the average energy required to produce an electron hole pair and $(\Delta E)_{\min }$ is the energy deposited by minimum ionizing particles per unit track length. As $(\Delta E)_{\min }$ of protons in silicon is $0.38 \mathrm{KeV} / \mu \mathrm{m}$ and $W$ of a-Si:H is $4.8 \mathrm{eV}$, the average number of e-h pairs produced from a minimum ionizing proton is 80 per micron.[4] This corresponds to $4000 \mathrm{e}-\mathrm{h}$ pairs for a $50 \mu \mathrm{m}$-thick layer. In a pixel array configuration with a typical pixel 
size of $\sim 300 \mu \mathrm{m}$ or smaller, the detector noise could be fewer than 200 electrons with good electronics, and the generated signal would be about 20 times larger than the noise.

\subsubsection{Effect of Dangling Bonds}

The dangling bonds are ionized by releasing the non-bonded electrons under the electric field and the atoms become positively charged. This fixed positive charge causes the electric field in the i-layer to drop linearly with distance. Hence, in order to have a fully depleted detector, a minimum bias is needed which increases proportionately to the density of dangling bonds, and the ionized dangling bond density, $N_{d}{ }^{*}$, is the major quantity of interest in fabricating good detectors. The maximum ionized dangling bond density under strong reverse bias has been empirically determined as roughly one third of the total dangling bond density, $N_{d}$.[5] Device quality a-Si:H has $N_{d}{ }^{*}$ of $5 \sim 10 \times 10^{14} \mathrm{~cm}^{-3}$.

The electric field profile inside the i-layer is given by the Poisson equation:

$$
\frac{d^{2} \Phi(x)}{d x^{2}}=-\rho=-\frac{q N_{d}^{*}}{\varepsilon_{o} \varepsilon_{\alpha S i}}
$$

where $\Phi(x)$ is electric potential, $\rho$ is electric charge density, $\varepsilon_{o}$ is the dielectric constant of vacuum and $\varepsilon_{a S i}$ is the relative dielectric constant of a-Si:H. The full depletion bias, $V_{f}$, is obtained as follows with the boundary conditions of $\Phi(0)=V_{f}$ and $\Phi(d)=0$, where $d$ is the i-layer thickness.

$$
V_{f}=\frac{q N_{d}^{*} d^{2}}{2 \varepsilon_{o} \varepsilon_{a S i}}
$$

Therefore, the magnitude of the applied bias necessary for depletion of the i-layer varies linearly with the ionized defect density $\left(N_{d}{ }^{*}\right)$ and is proportional to the square of the i-layer thickness. The $N_{d}{ }^{*}$ is determined conveniently by hole onset measurement. In this 
measurement, the $\mathrm{p}-\mathrm{i}-\mathrm{n}$ diodes are illuminated with highly absorbing light on the $\mathrm{n}$-side. The hole signal amplitudes are measured by varying the reverse bias applied to the diode. The hole signal would not be observed until the electric field extends to the n-side of the diode.

For a $50 \mu \mathrm{m}$ thick diode, with an ionized defect density of $5 \sim 7 \times 10^{14} \mathrm{~cm}^{-3}$, full depletion can be achieved at a minimum voltage of $1300 \mathrm{~V}$. In a normal $\mathrm{p}-\mathrm{i}-\mathrm{n}$ diode with the p-n doped layer thickness of 20-30 nm, application of such a large bias will result in a strong electric field at the $\mathrm{p}-\mathrm{i}$ interface near the electrode, which in turn will trigger a breakdown mechanism. Schemes to suppress the breakdown will be discussed in section 3.2 .

Also, a low density of dangling bonds is required for a large value of mean free path of charge carriers. The mobility-lifetime product, $\mu \tau$, determines the mean drift length, $l_{D}=\mu \tau E$ where $E$ is the electric field of the external bias. Since it is established that $\mu \tau N_{d}=$ constant $=2.5 \times 10^{8}$ in i-layers made using pure silane gas, a low dangling bond density leads to a high $\mu \tau .[6]$

\subsubsection{Residual Stress in a-Si:H Film}

The total residual stress in a-Si:H films is the sum of the internal stress and the thermal stress.[7] Device quality a-Si:H films have $350 \sim 400 \mathrm{MPa}$ of residual stress on the average. Linear thermal expansion coefficients, Young's moduli, and Poisson's ratio of a-Si:H and of various substrate materials are listed in Table III-1. From equation (2.3.1) in section 2.3.1, we know that the stress arising from thermal expansion mismatch is usually less than $10 \%$ of the total stress with a temperature difference of $250^{\circ} \mathrm{C}$. Therefore, the observed residual stress is mainly due to the large internal compressive stress. 
Table III-1. Mechanical and thermal parameters of amorphous silicon and common substrates. Values for crystalline silicon are average values over different crystallographic orientations.

\begin{tabular}{cccc}
\hline Material & $\begin{array}{c}\text { Young's modulus } \\
(\mathrm{GPa})\end{array}$ & Poisson's ratio & $\begin{array}{c}\text { Thermal expansion } \\
\text { coefficient } \\
\left(10^{-6}{ }^{\circ} \mathrm{C}^{-1}\right)\end{array}$ \\
\hline a-Si:H & 44 & & 3.5 \\
7059 glass & 68 & 0.28 & 4.6 \\
fused silica & 72 & 0.16 & 0.55 \\
C-Si & 150 & 0.2 & 2.5 \\
Kapton ${ }^{\circledR}$ & 3 & 0.28 & 30 \\
\hline
\end{tabular}

The origin of the internal compressive stress is not well understood. However, two microscopic models have been suggested and are widely accepted, one is the hydrogen incorporation model and the other is competition between constraints due to coordination and microvoids. The hydrogen incorporation model proposed by Stevens and Johnson and by Harbison et al. is believed to be the major cause of the compressive stress.[8,9] As mentioned in section 2.3.1, the most likely cause of compressive intrinsic stress is large quantities of impurities which are incorporated within the grown film either after deposition or during deposition behind the growing surface. Examples include the stress in SiO films evaporated onto glass which show a compressive stress of $\sim 30 \mathrm{MPa}$ when the partial pressure of residual water vapor in the evaporation chamber is high.[10] Therefore, the additional hydrogen as indiffusing impurity could explain the high compressive stress values observed in a-Si:H. The first process for hydrogen incorporation occurs simultaneously with the addition of silicon atoms to the random network at the growing surface. During this process some hydrogen is incorporated as the network is forming, but the hydrogen incorporated in this process needs not contribute to intrinsic stress since its 
incorporation occurs integrally with the formation of the rigid silicon network at the growing surface. However, the second process involves the invasive incorporation of hydrogen into the rigid random network and directly produces compressive intrinsic stress. Some excess hydrogen at the growing surface diffuses into the near surface region and chemically reacts with the rigid network. The hydrogen atom produces repulsive force between itself and its surrounding. The occurrence of this process and its consequences have been thoroughly established from studies of hydrogen migration in single-crystal silicon.[11,12] Similarly, it is anticipated in the rigid amorphous network that there are Si bonds that can be hydrogenated, with the gain in overall energy from forming Si-H bonds only partially offset by the loss to compressive strain energy.

The second model adopted the premise that the a-Si:H random network is intrinsically stressed because it is overconstrained. As indicated in section 2.1.1, the average coordination number, which is 4 , is higher than the most preferred coordination

number in the 3 -dimensional random network, $\sqrt{6}$. Hence, the network has to be ruptured to release the strain. Existence of microvoids, which are on the order of a few missing atoms in size, relieve the local strain by reducing the effective coordination number. Stress reduction is thus accompanied by increase in the density of dangling bonds and/or of silicon dihydride bonds $(=\mathrm{Si}<\mathrm{H}$ ), which form the inner surface of the microvoids. The silicon atom with dihydride configuration, whose effective coordination number is 2 , reduces the local strain in the silicon network and the macroscopic compressive stress.

\subsubsection{Relationship between Stress and Dangling Bond Density}

Extensive research has been done to reduce the residual stress by changing deposition parameters.[7, 13, 14] However, comparing results from various groups, we know that the maximum compressive stress appears under the same conditions in which the optimized electronic properties are obtained. Also, the control of growth parameters 
produced a trade-off between the ionized dangling bond density and the stress, although few data are available to compare directly the stress and defect density. Both of the above models explain this behavior. According to the first model, if we adjust the deposition conditions to minimize the hydrogen incorporation behind the growing surface, there will not be sufficient number of hydrogen atoms available to passivate dangling bonds. According to the second model, we can relax the amorphous network by changing the growth condition to have increased ion bombardment[15-17] or formation of polymeric radicals. $[18,19]$ However, either of these effects is known to introduce additional dangling bonds.

A series of $\mathrm{p}$-i-n diodes with $5 \mu \mathrm{m}$-thick $\mathrm{i}$-layer sandwiched in $0.1 \mu \mathrm{m}$-thick $\mathrm{p}$ - and n-layers was prepared and the stress and $N_{d}{ }^{*}$ were measured to see the relationship between them. The substrate temperature was varied between $150^{\circ} \mathrm{C}$ and $250^{\circ} \mathrm{C}$. All other conditions were fixed at the RF frequency of $85 \mathrm{MHz}, \mathrm{RF}$ power density of $40 \mathrm{~mW} / \mathrm{cm}^{2}$, chamber pressure of $300 \mathrm{mTorr}$. The total stress induced in a-Si:H films deposited on flat surfaces was calculated from eqn.(2.3.6) by measuring the curvature of the substrate.

The ionized dangling bond density was determined by hole onset measurement, in which the hole signal amplitude is measured while illuminating the $n$ - side of the $p-i-n$ diode at various applied bias voltages. The hole signal threshold bias corresponds to full depletion and is used to calculate the $N_{d}{ }^{*}$ as explained in the previous section.

The relationship between stress and $N_{d}{ }^{*}$ is plotted in Fig. 3.2. The deposition temperatures are indicated for each data points. The stress increased monotonously with decreasing $N_{d}{ }^{*}$. This result agrees well with other work which indicated that low stress materials have high $N_{d}{ }^{*}$ or vice versa.[20,21] The kink at the substrate temperature of $170^{\circ} \mathrm{C}$ indicates that both the stress and $N_{d}{ }^{*}$ undergo an abrupt transition around this temperature. It is consistent with other results that silicon dihydride bond $(=\mathrm{Si}<\mathrm{H})$ formation sets in below $190^{\circ} \mathrm{C}$, which indicates the existence of microvoids.[22] 


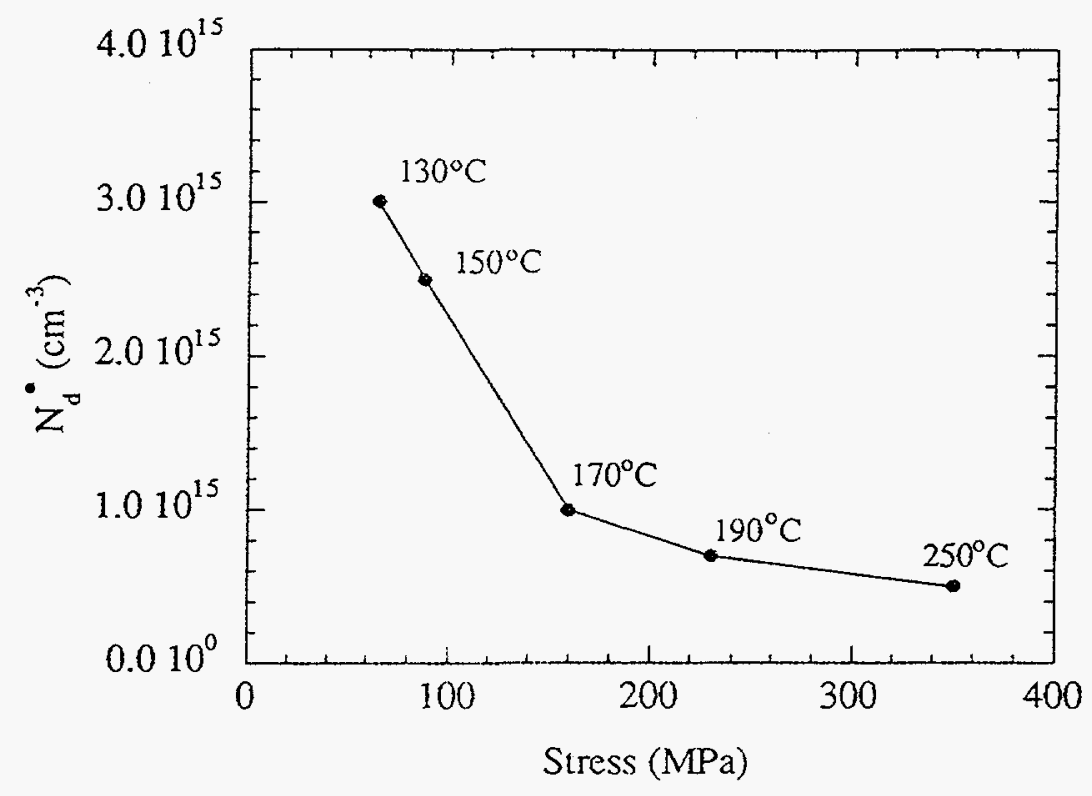

Figure 3.2. Relationship between the residual stress and ionized dangling bond density, $N_{d}{ }^{*}$, for a-Si:H films deposited at various temperatures. The deposition temperatures are indicated on each data points.

\subsubsection{Deposition Rate}

Slow growth rate of a-Si:H films by the PECVD technique often casts difficulty in making thick films. Typically the growth rate ranges from about $1 \sim 10 \AA / \mathrm{sec}$ and is approximately proportional to the RF power.[23] Dilution of silane with inert gas and high power facilitates the consumption of silane by the reaction. However, good quality material is grown far away from this condition.

Control of the morphology of the film is important for obtaining good electrical properties. Uniform, homogeneous layers free from macroscopic inhomogeneities, such as voids and columnar structure, are desired, since the inhomogeneities degrade the 
electronic properties. The film morphology depends on the deposition chemistry and on the surface mobility of the depositing molecules.[24]

There are two characters of the deposition process which influence the morphology and growth rate of the film.[15] One type of growth is chemical vapor deposition (CVD) which is governed by chemical reactions between the gas species and the growing surface. In this case, the gas has a low sticking coefficient and a high surface mobility.[23] The growth rate is therefore determined by the surface chemical reaction, rather than by the flux of radicals striking the surface. The high surface mobility of CVD growth results in a smooth conformal surface. The other type of growth is physical vapor deposition (PVD) which is governed by the flux of molecules striking the surface.[25] The gas has a high sticking coefficient and each molecule remains where it first strikes the surface.

In the plasma enhanced deposition, a PVD mechanism is favored when highly reactive gas species are formed in the plasma and the deposition rate is high, whereas less reactive species favor CVD.[26] Addition of argon into the plasma and a high RF power cause the deposition to be more PVD-like. PVD films have a high density of electronic defects which are associated with the internal surfaces of voids. When exposed to air, these voids allow the diffusion of oxygen and accelerates oxidation. The flat, conformal, CVD-like growth is important in the device technology to ensure that complex device structures maintain film continuity and uniformity. So far it is known that the best a-Si:H films are deposited at a slow rate from pure silane and with a low RF power. 


\subsection{FIELd PROFIle TAILORING}

In the previous section, obstacles to fabrication and operation of thick a-Si:H detectors have been addressed. Among them is the high reverse bias voltage required to fully deplete the detector. For a $50 \mu \mathrm{m}$ thick diode, usually the bias voltage required for full depletion is greater than $1000 \mathrm{~V}$. In a normal diode with $\mathrm{p}$ or $\mathrm{n}$ doped layer thickness of $20 \sim 30 \mathrm{~nm}$, application of such a high bias will result in the electric field peaking at the p-i interface which is close to the metal contact and will enhance breakdown. The highest reverse bias applicable to this type of devices is also limited to $10 \sim 20$ times the thicknesses of diodes in $\mu \mathrm{m}$ by the onset of a pop-corn type noise.[27, 28]

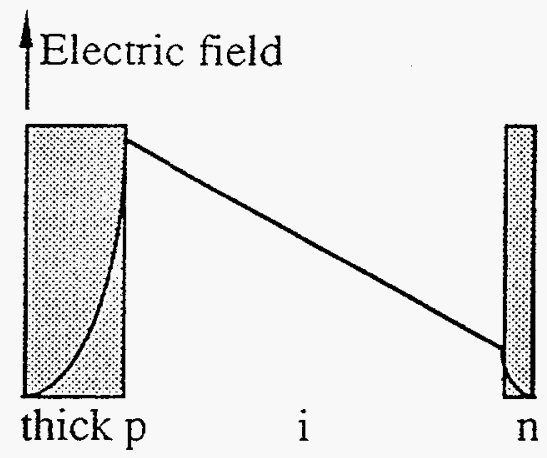

(a)

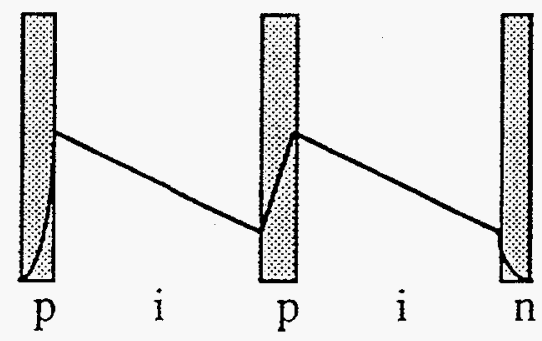

(b)

Figure 3.3. Full depletion schemes: (a) thick $p$ layer, (b) buried $p$ layer 
The peak field in the p-i interfaced can be sufficiently moved away from the contact region by increasing the thickness of the p layer to $200 \sim 300 \mathrm{~nm}$ as illustrated in Fig. 3.3(a). The depletion of the diode can be further promoted by depositing a thin p-layer in the middle of the intrinsic layer (see Fig. 3.3(b)). This layer will reduce the required depletion voltage approximately by a factor of 2.[29] At the same time, however, the player is expected to act as traps for electrons.

In this section, fabrication and characterization of this new configuration device will be presented, and the electron losses due to the buried p-layer will be estimated by a model calculation. Issues related to growth and mechanical stability of the thick layers will be discussed in sections $3.3 \sim 3.5$.

\subsubsection{Calculation of Electron Loss}

The fraction of electrons surviving after passsing the buried p-layer is calculated for a hypothetical structure of a $50 \mu \mathrm{m}$-thick diode having one p-layer in the middle as drawn in Fig. 3.4. Basic assumptions for this calculation are:

(a) $L$ is the total thickness of the detector diode.

(b) Thicknesses of $\mathrm{p}^{+}$and $\mathrm{n}^{+}$layers at both ends of the diode are thin enough to be neglected.

(c) The buried layer is located exactly in the middle of the i-layer.

(d) The doping density in the buried layer, $\left[\mathrm{B}_{4}\right]_{S}$, is decided in such a way that the maximum electric field inside the buried layer is the same as the field at the p-i interface at one end of the dectector. Hence, the configurations in both i-layers are exactly the same, i.e., $\left[\mathrm{B}_{4}\right]_{\mathrm{s}} \cdot d=\frac{L-d}{2} N_{d}^{*}$, where $d$ is the thickness of the buried p-layer.

(e) Finally, the applied bias, $V$, is equal to the area under the $E-x$ curve,

$$
V=2\left[\frac{1}{2}\left(E_{\max }+E_{\min }\right) \frac{L-d}{2}\right]+\frac{1}{2}\left(E_{\max }+E_{\min }\right) d=\frac{\left(E_{\max }+E_{\min }\right) L}{2}
$$




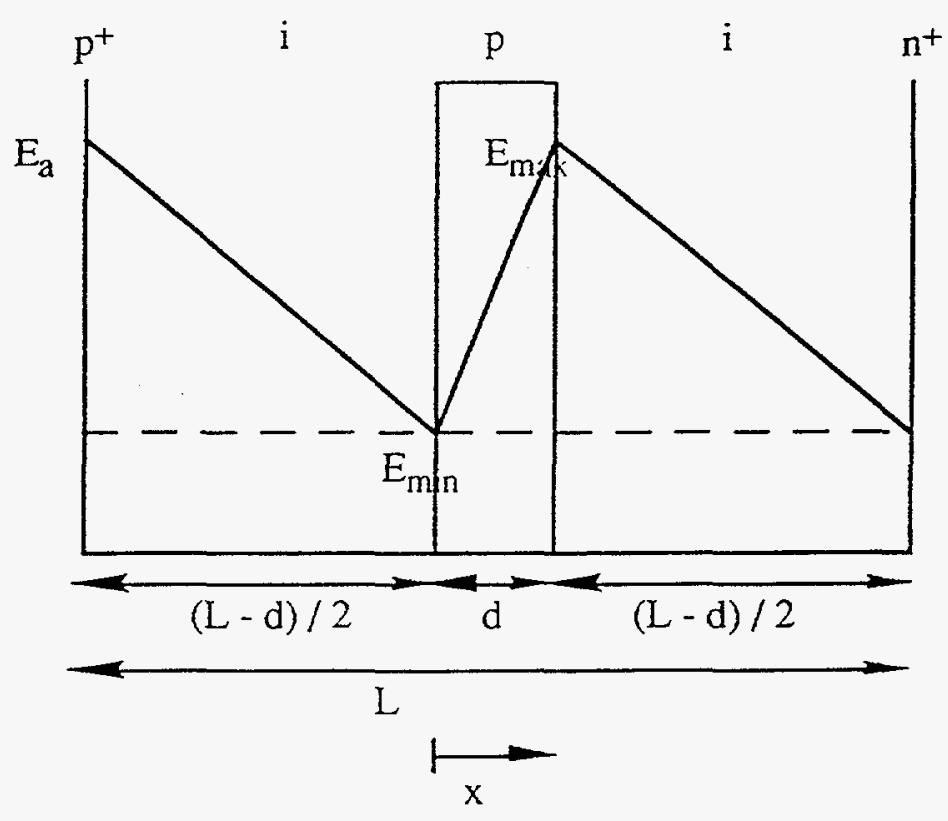

Figure 3.4. Electric field configuration of $\mathrm{p}^{+}-\mathrm{i}-\mathrm{p}-\mathrm{i}-\mathrm{n}^{+}$diode

The survival probability, $\eta$, is defined as follows:

$$
\eta=\frac{\text { number of electrons entering the buried } p \text {-layer }}{\text { number of electrons exiting the buried } p \text {-layer }}
$$

Variation of electron concentration along the distance in the p-layer, $x$, can be expressed as

$$
\frac{d n(x)}{d x}=-\frac{n(x)}{l_{D}}=-\frac{n(x)}{\mu \tau E(x)}
$$

where $n(x)$ is the number of electrons at position $x, x$ is the distance measured from the left-hand side intrinsic/buried layer interface, $l_{D}$ is the mean free path of the electrons in the p-layer, $\mu$ is the drift mobility of the electrons in the p-layer, $\tau$ is the lifetime of the electrons in the p-layer, and $E(x)$ is the electric field in the p-layer.

If the dopants are distributed uniformly in the buried p-layer, the electric field increases linearly with the distance $x$, and can be expressed in a simple form 


$$
E(x)=\frac{\left(E_{\max }-E_{\min }\right) x}{d}+E_{\text {min }}
$$

where $d$ is the thickness of the buried p-layer. Inserting eqn.(3.2.4) into eqn.(3.2.3) gives

$$
\frac{d n(x)}{d x}=-\frac{n(x)}{\mu \tau\left[\frac{\left(E_{\max }-E_{\min }\right) x}{d}+E_{\min }\right]}=-\frac{n(x)}{a x+b}
$$

where $a=\frac{\mu \tau\left(E_{\max }-E_{\min }\right)}{d}$, and $b=\mu \tau E_{\min }$

With a boundary condition $n(0)=n_{0}$, solution to the above differential equation (3.2.5) is

$$
n(x)=n_{o}\left(\frac{a x}{b}+1\right)^{-1 / a}=n_{o}\left[\frac{\left(E_{\max }-E_{\min }\right)}{E_{\min }} \frac{x}{d}+1\right]^{-\left\{d / \mu \tau\left(E_{\max }-E_{\min }\right)\right\}}
$$

where $n_{o}$ is the number of electrons entering the buried p-layer. From the eqn. (3.2.6), we can calculate the number of electrons at the exit of the buried p-layer which is $n(d)$. Therefore, the survival probability of signal electrons drifting the buried p-layer is

$$
\eta=\frac{n(d)}{n(0)}=\left(\frac{E_{\max }}{E_{\min }}\right)^{-\left\{d / \mu \pi\left(E_{\max }-E_{\min }\right)\right\}}
$$

Now, $\eta$ can be estimated if the values of $E_{\max }, E_{\min }$, and $\mu \tau$ are known. The electric field $E_{\max }$ and $E_{\min }$ are calculated in a simple case. Poisson's equations for the i-layers and the buried p-layer are

$$
\begin{aligned}
& \frac{d E(x)}{d x}=-\frac{q}{\varepsilon_{0} \varepsilon_{a S i}} N_{d}^{*} \quad \text { for } 0<x<\frac{L-d}{2} \text { and } \frac{L+d}{2}<x<L \quad \text { (Eqn. 3.2.8) } \\
& \frac{d E(x)}{d x}=-\frac{q}{\varepsilon_{0} \varepsilon_{a S i}}\left[\mathrm{~B}_{4}\right]_{\mathrm{s}} \quad \text { for } \frac{L-d}{2}<x<\frac{L+d}{2} \quad \text { (Eqn. 3.2.9) }
\end{aligned}
$$


From these relations we can calculate the magnitudes of $E_{\max }$ and $E_{\min }$. When the active boron doping concentration in the buried layer, $\left[\mathrm{B}_{4}\right]_{s}$, is given, $\mu \tau$ value of the electrons in the p-layer changes from that of the intrinsic a-Si:H layer. Street and coworkers reported the change in $\mu \tau$ values of electrons and holes with various doping gas concentrations $\chi_{g}$. [30] Stutzmann et al., reported a relationship between the active dopant density $\left[\mathrm{B}_{4}\right]_{S}$ and the gas phase concentration $\chi_{g}$.[31] Using linear regression, the following relationships have been obtained from the original data points in the literatures.

$$
\begin{aligned}
& \mu \tau=2.5 \times 10^{-12} \chi_{g}^{-0.51} \mathrm{~cm}^{2} / \mathrm{V} \\
& {\left[\mathrm{B}_{4}\right]_{\mathrm{S}}=10^{19} \chi_{g}^{0.37} \mathrm{~cm}^{-3}}
\end{aligned}
$$

Hence, $\mu \tau=3.9 \times 10^{14}\left[\mathrm{~B}_{4}\right]_{\mathrm{s}}{ }^{-1.38} \mathrm{~cm}^{2} / \mathrm{V}$

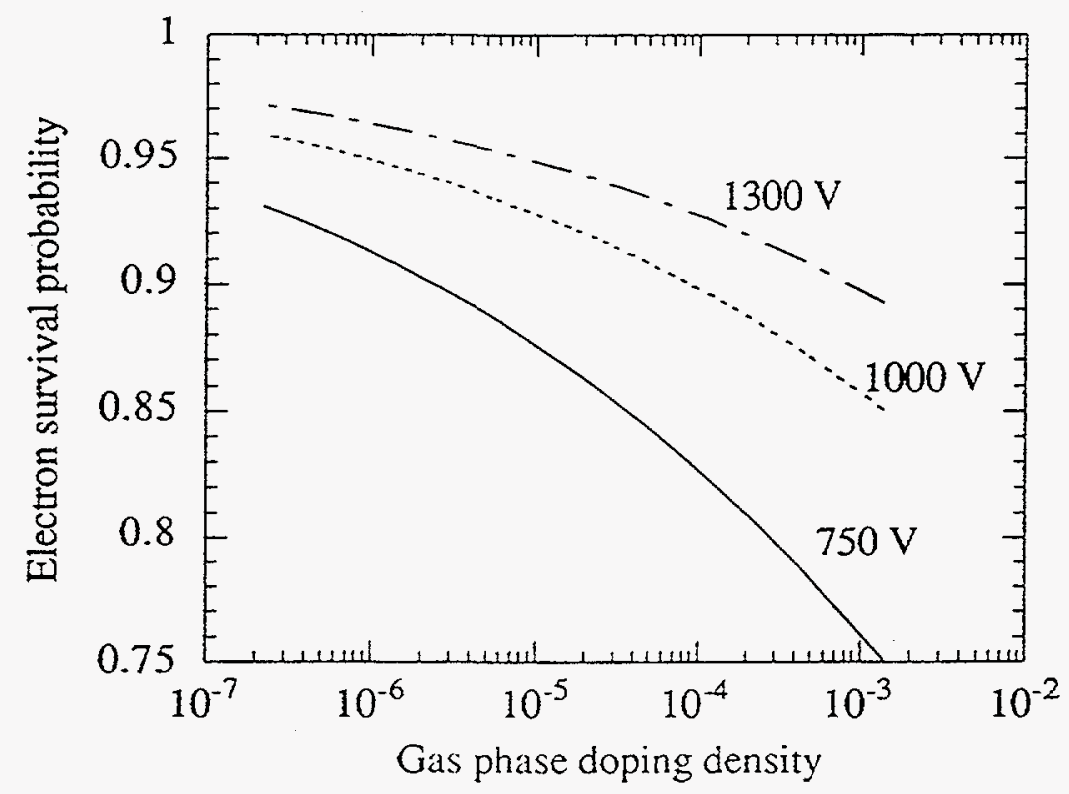

Figure 3.5. Calculated electron survival probability as a function of the gas phase doping density of the buried p-layer in a $50 \mu \mathrm{m}$ thick detector for 3 different bias voltages. 
In Fig. 3.5, the calculated electron survival probability, $\eta$, as a function of the doping density in the buried layer is plotted for various bias voltages. It is seen from the figure that more electrons are trapped as the buried layer doping density is higher. Since a higher doping is needed for a thinner buried layer to fully compensate the electric field drop, a wide and lightly doped layer is preferred to a narrow and heavily doped layer. It should also be noted that increasing the bias voltage also increases the collection efficiency. However, using a higher bias contradicts the purpose of the buried layer. A practical limit was set to a bias voltage of $750 \mathrm{~V}$ and a gas phase boron doping concentration of $10 \mathrm{ppm}$, which correspond to a bias slightly higher than the full depletion voltage of a $50 \mu \mathrm{m}$-thick buried p-layer diode and a minimum controllable gas flow rate with the current apparatus in LBL, respectively. As shown in the figure, for the above condition, the electron loss is less than $15 \%$.

\subsubsection{Measurement Results}

P-i-n diodes of a total thickness of $15 \mu \mathrm{m}$, having a buried p-layer in the middle were prepared by the PECVD technique under the conditions mentioned in section 2.1.5. The buried p-layer had a gas phase doping density of $114 \mathrm{ppm}$ and a thickness of $0.05 \mu \mathrm{m}$. Standard p-i-n diodes of the same thickness were also prepared for comparison. The voltage required to deplete the devices were measured by the hole onset technique described in section 3.1.2.

In Fig. 3.6, hole signals against applied bias are shown for two types of samples, one having no intermediate p-layer and the other having a p-layer in the middle. The depletion voltage was estimated by extrapolating the steeply rising part of the curve to the base level. In Fig. 3.6(b), the hole signal appears at nearly $1 / 2$ of the full depletion bias of a same-thickness standard diode. A feature of the curve is the discontinuity in the slope at the bias of $\sim 110 \mathrm{~V}$. This may arise because too many acceptor sites were present in the 
buried p-layer. For this reason the intermediate p-layer is not fully depleted when depletion of the intrinsic layers is complete. As a result additional bias appears only between the buried p-layer and the n-type layer. When depletion of the p-layer is complete, voltage appears across the whole detecting layer. In this case the rate of increase of field at the ntype layer with bias falls because the effective thickness has increased by a factor of 2 .

In order to check that electron losses in the devices were as expected, measurements of mobility-lifetime product, $\mu \tau$, were carried out. The method has been described in section 2.1.4. The $\mu \tau$ value obtained from eqn. (2.1.9) is an averaged quantity, $(\mu \tau)_{\text {eff }}$ such that

$$
\frac{1}{(\mu \tau)_{e f f}}=\frac{1}{(\mu \tau)_{i}}+\frac{1}{(\mu \tau)_{p}}\left(\frac{d}{L}\right)
$$

where $(\mu \tau)_{i}$ and $(\mu \tau)_{p}$ are the $\mu \tau$ values in the intrinsic layer and in the p-type layer, respectively, and $d$ and $L$ are the thicknesses of the p-type layer and the intrinsic layer, respectively, as indicated in Fig. 3.4. The values of $\mu \tau$ found were $1.1 \times 10^{-7} \mathrm{~cm}^{2} / \mathrm{V}$ for the sample with no buried p-layer and $2.5 \times 10^{-8} \mathrm{~cm}^{2} / \mathrm{V}$ for samples with a buried p-layer. Using eqn. (3.2.13), $(\mu \tau)_{p}$ is determined to be $1.1 \times 10^{-10} \mathrm{~cm}^{2} N$. This gives a value of $N_{A}(\mu \tau)_{p}$ of $1.1 \times 10^{7} \mathrm{~V}^{-1} \mathrm{~cm}^{-1}$ where $N_{A}$ is the density of acceptor atoms in the p-layer. This value is somewhat lower than the value reported by Street, $3.5 \times 10^{8}$.[32] However, since measurements of $\mu \tau$ often vary by $50 \%$ between samples, this value is within reasonable bound. 


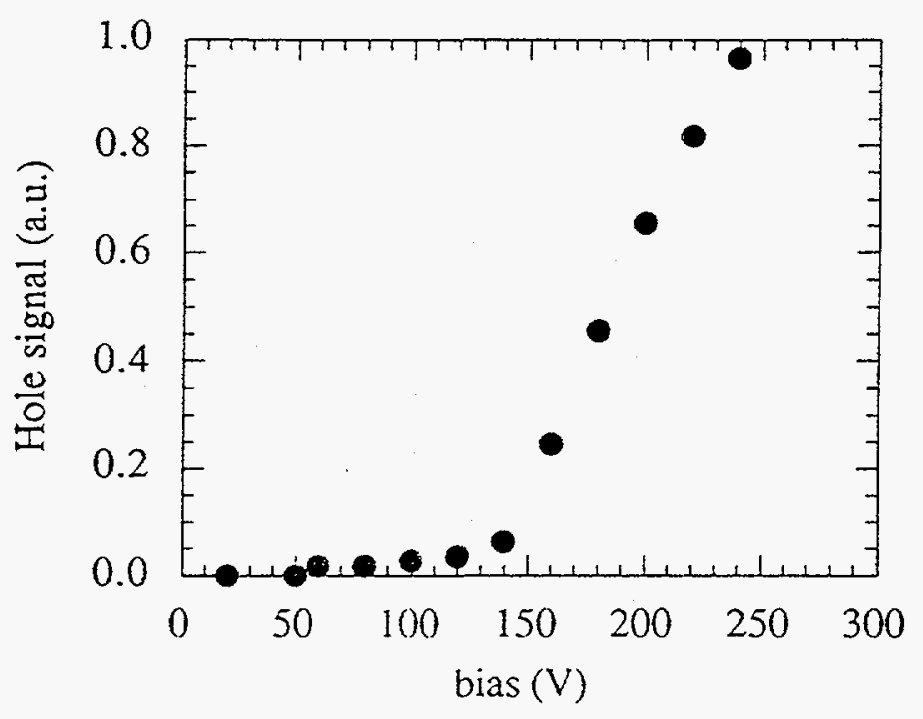

(a)

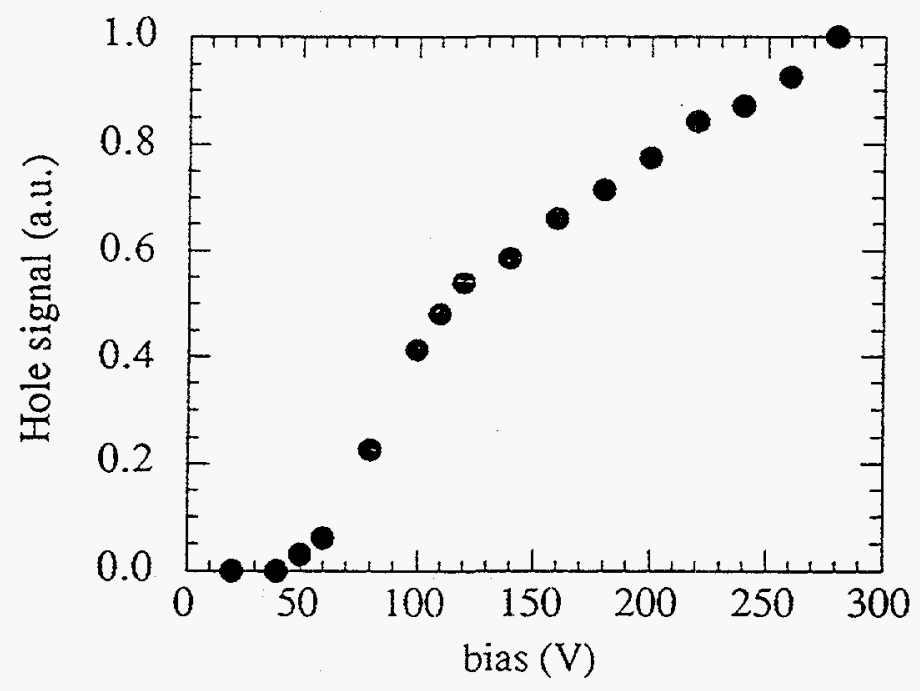

(b)

Figure 3.6. Hole signal (arbitrary unit) vs. bias voltage for (a) $\mathrm{p}-\mathrm{i}-\mathrm{n}$ diode with no buried p-layer, and (b) p-i-n diode with a buried p-layer in the middle. 


\subsection{GROWTH OF THICK LAYERS}

In section 3.1, material and process parameters to be considered in making thick, high quality a-Si:H layers were addressed. The deposition of such layers by the conventional a-Si:H technology (glow-discharge of $\mathrm{SiH}_{4}$ at a $\mathrm{RF}$ frequent of $13.56 \mathrm{MHz}$ and a temperature of $250^{\circ} \mathrm{C}$ ) is handicapped by the high residual stress and low deposition rate. In this section, solutions to overcome each obstacle are described. The goal is to achieve: i) high deposition rate, ii) low residual stress, iii) a low ionized dangling bond density and iv) good mechanical stability.

\subsubsection{Increased Deposition Rate}

(1) Higher RF Frequency :

Growth rate of a good quality intrinsic layer at the conventional plasma excitation frequency of $13.56 \mathrm{MHz}$ is $\sim 0.7 \mu \mathrm{m} / \mathrm{hr}$. However, higher PECVD deposition rate of intrinsic a-Si:H films is required for commercial applications such as, low cost solar cells and production of thick detectors. Increased growth rate can be achieved by the use of high plasma excitation frequencies in the Very High Frequency (VHF) band $(30 \sim 300 \mathrm{MHz})$. Application of excitation frequencies in the $10 \sim 110 \mathrm{MHz}$ range has been shown to have no deterioration effect on the electronic properties of the deposited film.[33, 34] High deposition rates are believed to be the results of the surface hydrogen desorption due to the high flux of low energetic ions to the growth surface and of the enhanced probability of highly reactive radicals reaching the growth surface due to a decreased sheath thickness. $[35,36]$ The existing equipment at LBL was modified to house higher frequencies and the excitation frequency was boosted from $13.56 \mathrm{MHz}$ to $85 \mathrm{MHz}$. At this frequency, the deposition rate of standard a-Si:H films has increased to $2.3 \mu \mathrm{m} / \mathrm{hr}$. 
(2) Helium Dilution of Silane :

Further improvement in the growth rate can be achieved by introducing inert gas to the silane. Dilution of silane with inert gas enhances ion bombardment and dissociation of silane into reactive radicals during deposition.[16, 17, 37] The enhanced dissociation facilitates the diffusion of radicals to the growing surface and the increased ion bombardement etches away weakly bonded atoms. It has been reported that the deposition rate of the amorphous silicon film can be increased by mixing silane with helium.[38, 39] However, the reported value of $N_{d}{ }^{*}$ in the He-diluted i-layer was about 2 times larger than that in the standard a-Si:H materials. Hence, modification of the deposition parameters was performed to achieve both a moderate enhancement in the deposition rate and a reduction in the $N_{d}{ }^{*}$. The deposition parameters of the He-diluted and conventional a-Si:H are summarized in Table III-2. All samples were prepared at the plasma excitation frequency of $85 \mathrm{MHz}$.

Table III-2. Deposition Parameters for Conventional and He-diluted a-Si:H

\begin{tabular}{clcccc}
\hline Sample ID & Gas & Temp. $\left({ }^{\circ} \mathrm{C}\right)$ & $\begin{array}{c}\text { Power } \\
\text { Density } \\
\left(\mathrm{mW}^{2} \mathrm{~cm}^{2}\right)\end{array}$ & $\begin{array}{c}\text { Pressure } \\
(\mathrm{mTorr})\end{array}$ & $\begin{array}{c}\text { Deposition } \\
\text { Rate } \\
(\mu \mathrm{m} / \mathrm{hr} .)\end{array}$ \\
\hline Standard & $100 \% \mathrm{SiH}_{4}$ & 250 & 40 & 300 & 2.3 \\
He338 & $40 \% \mathrm{SiH}_{4}-60 \% \mathrm{He}$ & 250 & 40 & 500 & 2.5 \\
He339 & $40 \% \mathrm{SiH}_{4}-60 \% \mathrm{He}$ & 250 & 90 & 300 & 2.4 \\
He340 & $40 \% \mathrm{SiH}_{4}-60 \% \mathrm{He}$ & 250 & 90 & 550 & 4.4 \\
He341 & $10 \% \mathrm{SiH}_{4}-90 \% \mathrm{He}$ & 250 & 90 & 550 & 3 \\
He356 & $40 \% \mathrm{SiH}_{4}-60 \% \mathrm{He}$ & 250 & 90 & 500 & 3.9 \\
He357 & $40 \% \mathrm{SiH}_{4}-60 \% \mathrm{He}$ & 250 & 40 & 300 & 2.2 \\
He359 & $40 \% \mathrm{SiH}_{4}-60 \% \mathrm{He}$ & 300 & 40 & 300 & 2.2 \\
\hline
\end{tabular}


Table III-3. Measured Electron Transport Parameters and Stress of the Hediluted and Conventional a-Si:H

\begin{tabular}{ccccccc}
\hline Sample ID & $\begin{array}{c}\mu_{\mathrm{e}} \\
\left(\mathrm{cm}^{2} / \mathrm{N} \cdot \mathrm{sec}\right)\end{array}$ & $\begin{array}{c}(\mu \tau)_{\mathrm{e}} \\
\left(\mathrm{cm}^{2} N\right)\end{array}$ & $\begin{array}{c}\mu_{\mathrm{h}} \\
\left(\mathrm{cm}^{2} \mathrm{~N} \cdot \mathrm{sec}\right)\end{array}$ & $\begin{array}{c}(\mu \tau)_{\mathrm{h}} \\
\left(\mathrm{cm}^{2} / \mathrm{N}\right)\end{array}$ & $\mathrm{N}_{\mathrm{d}}^{*}\left(\mathrm{~cm}^{-3}\right)$ & $\begin{array}{c}\text { Stress } \\
(\mathrm{MPa})\end{array}$ \\
\hline Standard & 1 & $7.3 \times 10^{-8}$ & 0.002 & $2.8 \times 10^{-8}$ & $7 \times 10^{14}$ & 350 \\
He338 & 1.6 & $5.6 \times 10^{-8}$ & 0.002 & $7.5 \times 10^{-8}$ & $2 \times 10^{14}$ & 320 \\
He339 & 2.4 & $9 \times 10^{-9}$ & - & - & $2 \times 10^{14}$ & 470 \\
He340 & 1.6 & $5.3 \times 10^{-8}$ & - & - & $3.3 \times 10^{14}$ & 280 \\
He341 & 2.2 & $3.8 \times 10^{-8}$ & 0.002 & $7.9 \times 10^{-8}$ & $0.4 \times 10^{14}$ & 540 \\
He356 & 1.6 & $3.4 \times 10^{-8}$ & 0.002 & $1.3 \times 10^{-7}$ & $2.5 \times 10^{14}$ & 320 \\
He357 & 1.8 & $1.4 \times 10^{-8}$ & - & - & $1.8 \times 10^{14}$ & 320 \\
He359 & 1.3 & $9 \times 10^{-9}$ & - & - & $1.3 \times 10^{14}$ & 340 \\
\hline
\end{tabular}

Electron and hole mobilities were measured by the standard time-of-flight method. The mobility-lifetime products of electron and hole were measured by collecting transient charges at difference bias voltages. Hole onset measurement was performed to estimate the ionized dangling bond density. The backsides of the substrate was scanned with a profilometer and the residual stress was determined from the substrate curvature. Details of all the characterization techniques mentioned above are described in Chapter 2. The characterization results of material properties are listed in Table III-3.

From Table III-2, we see that the deposition rate increased with pressure and power. However, increase in deposition rate was sometimes accompanied by degradation in dangling bond density or carrier mobility. As seen from Table III-3, higher helium dilution led to a lower dangling bond density and higher stress, with a lower deposition rate. In most cases, He-dilution showed exceptionally low ionized dangling bond density and high hole $\mu \tau$, with relatively poor electron $\mu \tau$. This is indicative of compensation of 
the slightly n-type nature of intrinsic a-Si:H, which is due to ionization of dangling bonds, by displacement of the Fermi level towards the valence band.

With all the characteristics taken into account, an optimum deposition condition was chosen to be gas mixing ratio of $40 \% \mathrm{SiH}_{4}-60 \% \mathrm{He}$, chamber pressure of $500 \mathrm{mTorr}$, plasma power density of $90 \mathrm{~mW} / \mathrm{cm}^{2}$, and substrate temperature of $250^{\circ} \mathrm{C}$. A growth rate of $3.5 \sim 4 \mu \mathrm{m} / \mathrm{hr}$., and an $N_{d}^{*}$ of $2.5 \times 10^{14} \mathrm{~cm}^{-3}$ were obtained, which were improvements by factors of 1.5 and 3 , respectively, compared to the conventional a-Si:H. Material properties of the He-diluted films will be discussed in section 3.4 in more detail.

\subsubsection{Heat Treatment for Low Defect Density}

It is well established that the light-induced defects in a-Si:H can be reduced by annealing at a temperature below the growth temperature. $[40,41]$ Therefore, postdeposition heat treatment can be a good way to lower the increased defect density due to the change in deposition conditions towards a low stress material. The defect density in undoped a-Si:H has been found to reach a themal equilibrium in the temperature range of $100^{\circ} \mathrm{C}$ and $300^{\circ} \mathrm{C}$.[42-44] McMahon and Tsu found that the thermal equilibrium temperature, $T_{E}$, was $190 \sim 200^{\circ} \mathrm{C}$ in undoped a-Si:H.[45] The thermal equilibrium defect density increases with temperature, and the high-temperature state above $T_{E}$ can be frozen in by quenching to room temperature where the relaxation time is extremely long.[46, 47]

McMahon proposed a multivalley energy configuration coordinate diagram with small barriers between metastable states to describe defect states in a-Si:H films.[48] Figure 3.7 shows the multivalley energy configuration with two metastable states, $M_{1}$ and $M_{2}$, and the stable state $S_{0}$. This figure is slightly modified from the original diagram suggested by McMahon to reflect a new finding that the energy barrier between the metastable states is comparable to that between the stable and metastable states. 


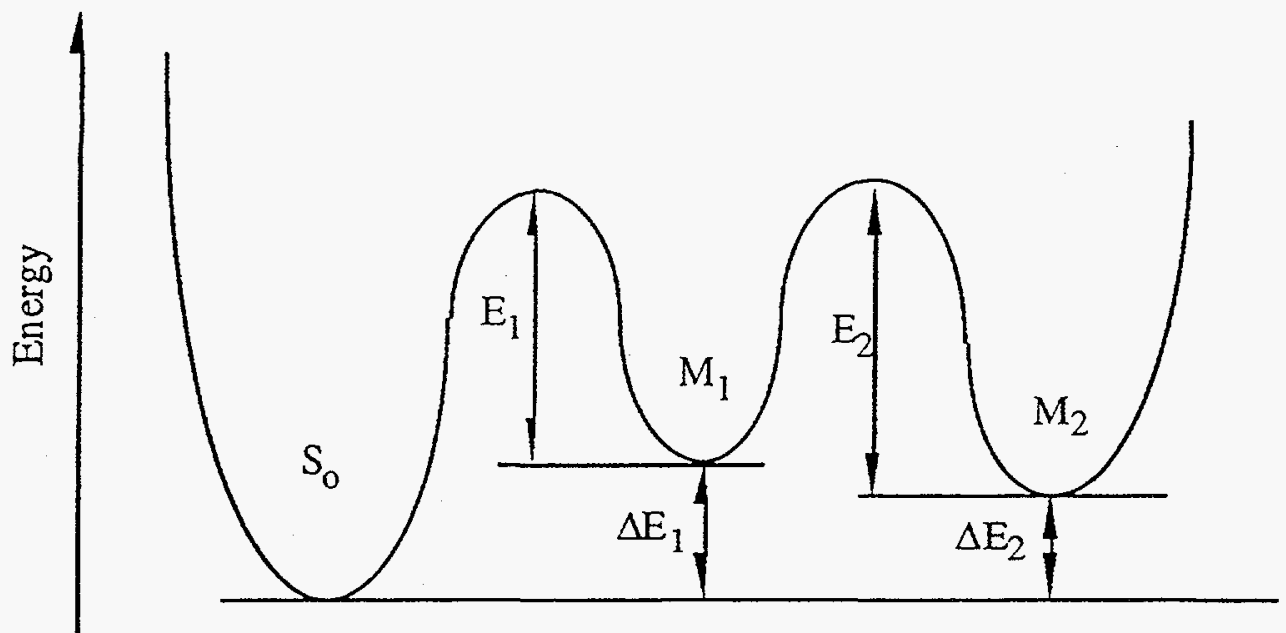

Figure 3.7. Diagram of multivalley energy configuration showing a stable state $S_{0}$, and metastable states, $M_{1}$ and $M_{2}$.

A-Si:H p-i-n diodes with $14 \mu \mathrm{m}$-thick i-layers were deposited on chromium-coated Corning 7059 glass substrates at $250^{\circ} \mathrm{C}$. Other deposition parameters were the same as those in section 2.1.5. At first, the samples were heated to $300^{\circ} \mathrm{C}$ for 10 minutes and then fast-cooled by immersing in water at room temperature. Dangling bond density and residual stress were measured and these values were used as references. The samples were then annealed at temperatures $160^{\circ} \mathrm{C}, 200^{\circ} \mathrm{C}$, and $250^{\circ} \mathrm{C}$ for different lengths of time and were quenched again. Transient photoconductivity and stress measurements were performed according to procedure described in Chapter 2. Mobility-lifetime product, $\mu \tau$, was estimated by the transient photoconductivity measurement and the defect density was extracted from the relation, $\mu \tau N_{d}=2.5 \times 10^{8}$.[6]

The relaxation data for defect density are shown in Fig. 3.8. The $N_{d}$ decreased by factors of $3 \sim 5$ according to the annealing temperature. The higher the annealing temperature, the shorter it takes to reach an equilibrium value but the smaller the extent of relaxation. All curves showed the stretched exponential behavior and have minimum values prior to their equilibrium. 
Earlier studies have used the following stretched single-term exponential equation to analyze the annealing data. $[49,50]$

$$
N_{d}(t)=N_{f}+\left(N_{i}+N_{f}\right) \exp \left\{-\left(t / t_{R}\right)^{\beta}\right\}
$$

where $N_{i}$ is the initial quenched-in defect density, $N_{f}$ is the final annealed density, $t_{R}$ is the relaxation time constant, and $\beta$ is the dispersion parameter. However, the above equation does not fit very well to the experiment data because of the presence of the minimum value before equilibrium.[51] Therefore, the equation (3.3.1) is modified according to the multivalley configuration model in Fig. 3.7.

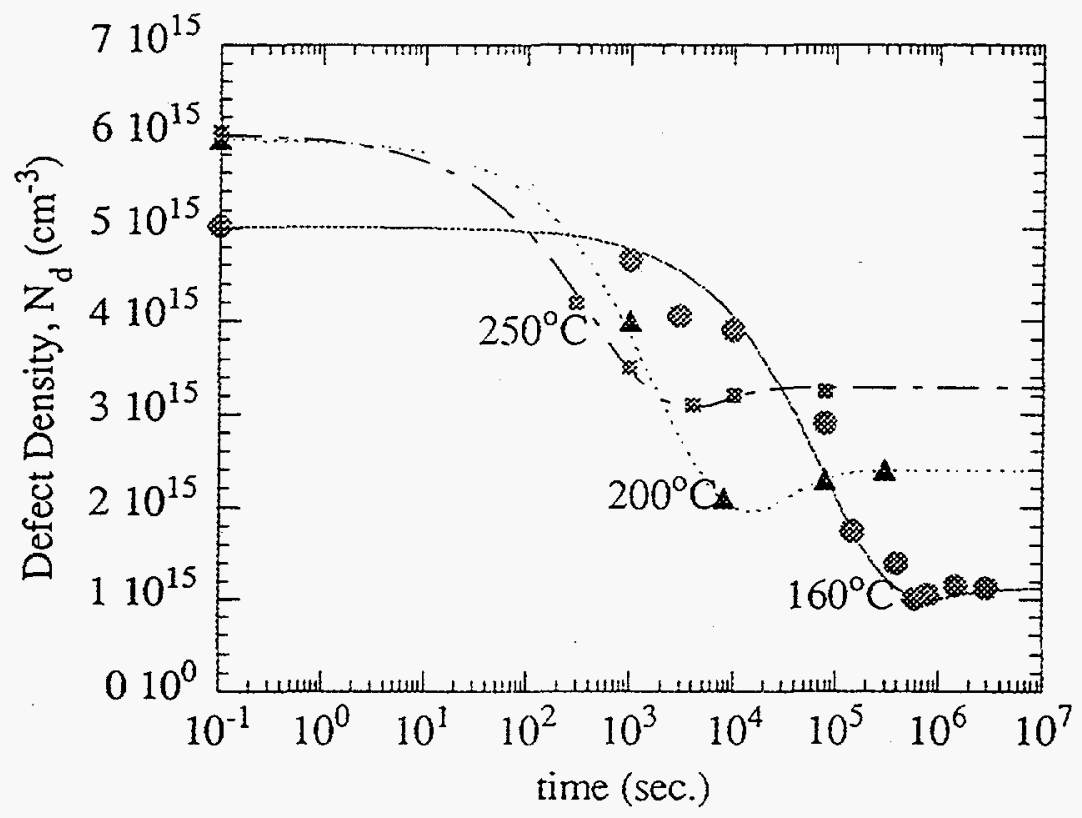

Figure 3.8. Time dependence of defect density during annealing at different temperatures. Circles, triangles and squares represent the measured defect densities with annealing temperatures of $160^{\circ} \mathrm{C}, 200^{\circ} \mathrm{C}$, and $250^{\circ} \mathrm{C}$, respectively. The samples were degraded by heating at $300^{\circ} \mathrm{C}$ for 10 minutes and quenched in water prior to annealing. The lines are the results from curve fitting with eqn.(3.3.7) 


$$
N_{d}(t)=N_{1}(t)+N_{2}(t)
$$

where $N_{1}(t)$ and $N_{2}(t)$ are the defect densities at the metastable states $M_{1}$ and $M_{2}$. Both $N_{1}(t)$ and $N_{2}(t)$ take the same stretched exponential form as eqn. (3.3.1):

$$
N_{\mathrm{I}}(t)=N_{f 1}+\left(N_{i 1}+N_{f 1}\right) \exp \left\{-\left(t / t_{R 1}\right)^{\beta 1}\right\}
$$

and $\quad N_{2}(t)=N_{f 2}+\left(N_{i 2}+N_{f 2}\right) \exp \left\{-\left(t / t_{R 2}\right)^{\beta 2}\right\}$

where $N_{i 1}$ and $N_{i 2}$ are the initial quenched-in spin densities, $N_{f 1}$ and $N_{f 2}$ are the final annealed values, $t_{R 1}$ and $t_{R 2}$ are the relaxation time constants, and $\beta_{1}$ and $\beta_{2}$ are the dispersion parameters at $M_{1}$ and $M_{2}$, respectively. Here, $N_{i}$ and $N_{f}$ are written as:

$$
\text { and } \quad \begin{aligned}
N_{i} & =N_{i 1}+N_{i 2} \\
N_{f} & =N_{f 1}+N_{f 2}
\end{aligned}
$$

Table III-4. Curve fitting parameters used in Figure 3-8

\begin{tabular}{cccc}
\hline annealing temperature & $160^{\circ} \mathrm{C}$ & $200^{\circ} \mathrm{C}$ & $250^{\circ} \mathrm{C}$ \\
\hline $\mathrm{N}_{\mathrm{i}}\left(10^{15} \mathrm{~cm}^{-3}\right)$ & 5.02 & 5.97 & 6.04 \\
$\mathrm{~N}_{\mathrm{f}}\left(10^{15} \mathrm{~cm}^{-3}\right)$ & 1.12 & 2.39 & 3.29 \\
$\mathrm{x}_{\mathrm{T}}$ & 0.40 & 0.45 & 0.30 \\
$\mathrm{t}_{\mathrm{R} 1}(\mathrm{sec})$. & $8 \times 10^{4}$ & $2 \times 10^{3}$ & $5.5 \times 10^{2}$ \\
$\mathrm{t}_{\mathrm{R} 2}(\mathrm{sec})$. & $8 \times 10^{5}$ & $2.5 \times 10^{4}$ & $4 \times 10^{3}$ \\
$\beta_{1}$ & 0.65 & 0.65 & 0.60 \\
$\beta_{2}$ & 0.80 & 0.80 & 0.80 \\
\hline
\end{tabular}


Since the first heat treatment at $300^{\circ} \mathrm{C}$ for making high temperature quenched-in defects were performed for a relatively short period, $N_{i 2}$ can be neglected. Thus, eqn. (3.3.2) can be expressed as the following two-term exponential form:

$$
\begin{aligned}
& N_{d}(t)=N_{f}+\left\{N_{i}-\left(1-x_{T}\right) N_{f}\right\} \exp \left[-\left(t / t_{R 1}\right)^{\beta 1}\right]-x_{T} N_{f} \exp \left[-\left(t / t_{R 2}\right)^{\beta 2}\right] \\
& \text { with } x_{T}=N_{f 2} /\left(N_{f 1}+N_{f 2}\right)
\end{aligned}
$$

Then the annealing data fit well to eqn. (3.3.7) and the fitting curves are shown in Fig. 3.8. The curve fitting parameters used in the figure are listed in Table III-4.

The physical meaning of the additional metastable state, $M_{2}$, can be explained as follows. When heated at high temperature, hydrogen is released from an $\mathrm{Si}-\mathrm{H}$ bond and creates a metastable state, $M_{1}$, by breaking a weak Si-Si bond and create a new Si-H bond.[44] During low temperature annealing, however, mobile hydrogen atom, which has created an $\mathrm{Si}-\mathrm{H}$ bond at the weak $\mathrm{Si}-\mathrm{Si}$ bond site, is released again and breaks another weak bond. Then a new metastable state, $M_{2}$ is created, and the broken Si-Si bond at the $M_{1}$ site is healed.

Metastable configurations of nearly the same energy $\left(M_{1}\right.$ and $M_{2}$ in Figure 3.7) become more locked in configurationally as more atoms become involved in the stabilization process. A quench from the $300^{\circ} \mathrm{C}$ equilibrium state might have the two valleys $M_{1}$ and $M_{2}$ with equal occupation. Further annealing at low temperatures could thermalize the system into the lower of the two metastable defect configurations $\left(M_{2}\right)$ favoring more locked in at the right. Some dangling bonds will anneal away rapidly, and an anneal of the remaining ones from the right take longer. It is possible that there are more metastable states $\left(M_{3}, M_{4}, \ldots\right)$, but they are not considered in this modeling. 


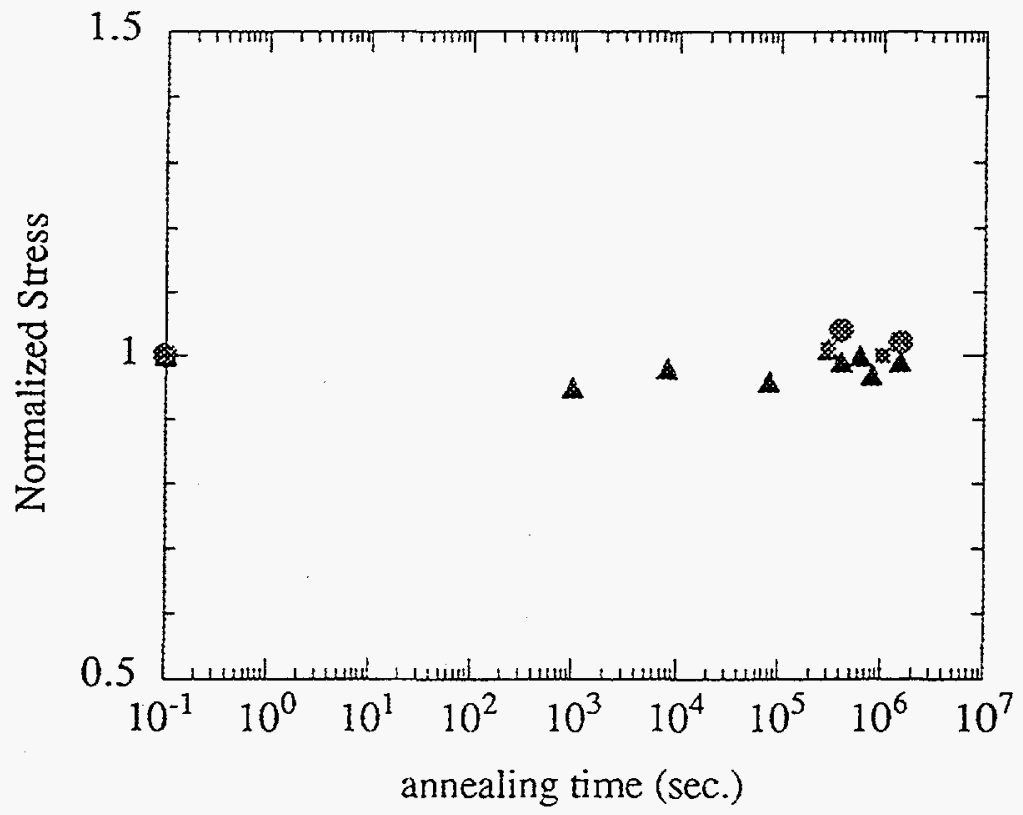

Figure 3.9. Time dependence of normalized stress, $\sigma(t) / \sigma(0)$, during annealing at different temperatures. Circles, triangles and squares represent the samples annealed at temperatures of $160^{\circ} \mathrm{C}, 200^{\circ} \mathrm{C}$, and $250^{\circ} \mathrm{C}$, respectively. The samples were degraded by heating at $300^{\circ} \mathrm{C}$ for 10 minutes and quenched in water prior to annealing.

The time dependence of residual stress values normalized to the initial stress is shown in Fig. 3.9. The stress remained constant during annealing in all cases within the experimental uncertainty. Considering the origin of stress and defect creation models, one can conclude that the change in stress and defect density is due to the Si-Si and Si-H bond structures. According to the models, low temperature annealing $\left(<250^{\circ} \mathrm{C}\right)$ only rearranges hydrogen and does not affect the number of $\mathrm{Si}-\mathrm{H}$ bonds or presence of microvoids. Therefore, changes in metastable state density, which are caused by hydrogen diffusion, are not sufficient to cause structural change, which is responsible for the internal stress, at temperatures between $160^{\circ} \mathrm{C}$ and $250^{\circ} \mathrm{C}$. 


\subsubsection{Adhesion}

If interfacial shear stresses exceed the adhesion strength, interfacial cracking and delamination of the film can result. Therefore, various types of substrates were tested for good adhesion and mechanical stability. Small pieces of crystalline silicon and Corning 7059 glass were coated with thin $\mathrm{Cr}$ or indium tin oxide (ITO) layers. These substrates were put into the deposition chamber together with uncoated specimens and a piece of molybdenum plate, and a-Si:H layers of 5 to $20 \mu \mathrm{m}$ in thicknesses were deposited under the standard condition described in section 2.1.5. Table III-5 lists adhesion results for various substrates. From this table, the ITO-coated substrates have the best adhesion property among the materials tested. For $20 \mu \mathrm{m}$-thick samples with residual stress of $\sim 350$ $\mathrm{MPa}$, further delamination did not occur even when part of the sample was removed by scratching, which is not the case in other substrates. The $\mathrm{Cr}$-coated substrates also have good adhesion, although inferior to the ITO-coated ones. However, except when good mechanical stability is more important than other characteristics, $\mathrm{Cr}$ layer is preferred as a bottom contact of a device, because the ITO contact leads to large low-frequency noise and low breakdown voltages.[28]

Another observation in the adhesion test is that there is a large run-to-run variation. Therefore, we know that the mechanical stability is not solely determined by the bonding strength between the film and the substrate. In general, second phase materials or contaminants at the film / substrate interface can inhibit the bonds between the film and the substrate. Water vapor or other gases from the ambient adsorbed on the substrate surface also weaken the surface bonding.[52] These broken bonds at the interface can be regarded as cracks which result in buckling. 
Table III-5. Adhesion of a-Si:H films deposited on various substrates

\begin{tabular}{ccccc}
\hline $\begin{array}{c}\text { Substrate } \\
\text { Material }\end{array}$ & $5 \mu \mathrm{m}$ & $10 \mu \mathrm{m}$ & $15 \mu \mathrm{m}$ & $20 \mu \mathrm{m}$ \\
\hline c-Si, uncoated & $\Delta$ & $\mathrm{X}$ & $\mathrm{X}$ & $\mathrm{X}$ \\
c-Si, Cr-coated & $\mathrm{O}$ & $\mathrm{O}$ & $\mathrm{O}$ & $\Delta$ \\
glass, uncoated & $\mathrm{X}$ & $\mathrm{X}$ & $\mathrm{X}$ & $\mathrm{X}$ \\
glass, Cr-coated & $\mathrm{O}$ & $\mathrm{O}$ & $\mathrm{O}$ & $\Delta$ \\
glass, ITO coated & $\mathrm{O}$ & $\mathrm{O}$ & $\mathrm{O}$ & $\mathrm{O}$ \\
molybdenum & $\mathrm{O}$ & $\Delta$ & $\Delta$ & $\mathrm{X}$ \\
\hline
\end{tabular}
O: stable
$\Delta$ : partially peeled off
X: Fully peeled off

The behavior of spontaneous peeling-off at small thicknesses due to propagation of interfacial cracking is analyzed following the original work by Evans and Hutchinson.[53] The driving force for crack propagation is characterized by a strain energy release rate, $G$, defined as follows

$$
G=\frac{\partial(W-U)}{\partial A}
$$

where $A$ is the area of the crack, $W$ is the work done by applied loads, and $U$ is the elastic energy of the system. The energy difference $\delta U=(W-U)$ can be estimated by modeling the delaminated region as a clamped circular plate of radius $a$. The strain energy release rate of a buckled film due to a penny-shaped crack is[54]:

$$
G=\frac{1}{2 \pi a} \frac{\partial \delta U}{\partial a}
$$


Now, imagine that the detached region has been separated from its surroundings. The radius expands due to relief of the biaxial compression, $\sigma_{o}$, by

$$
\delta a=\frac{(1-v) \sigma_{o} a}{E}
$$

If the compressive edge stress to force the film back to its original radius, $a$, is $\sigma$, the film will undergo buckling if $\sigma$ exceeds the critical stress $\sigma_{c}$.[55]

$$
\sigma_{c}=\frac{k E}{12\left(1-v^{2}\right)}\left(\frac{t}{a}\right)^{2}
$$

where $k=14.68$. The associated critical inward radial displacement is

$$
\Delta_{c}=\frac{k}{12(1+v)} \frac{t^{2}}{a}
$$

A plot of relative edge stress $\sigma / \sigma_{c}$ against the relative inward radial displacement of the edge $\Delta / \Delta_{c}$ looks like the one shown in Fig. 3.10. Solutions for both unbuckled and buckled configuration can be approximated by a straight line. The slope of the unbuckled branch is unity, while that of the post-buckled branch is

$$
\alpha=\frac{1}{1+1.207(1+v)}
$$

The strain energy change as a result of the stress redistribution due to buckling can be obtained from the area difference between the two curves, i.e., the shaded area in Fig. 3.10. By requiring that $\Delta=\delta a$ and using eqn.(3.3.11), the strain energy difference and the resulting energy release rate are expressed as

$$
\delta U=\frac{\pi(1-v)(1-\alpha)\left(\sigma_{o}^{2}-\sigma_{c}^{2}\right) t a^{2}}{E}
$$




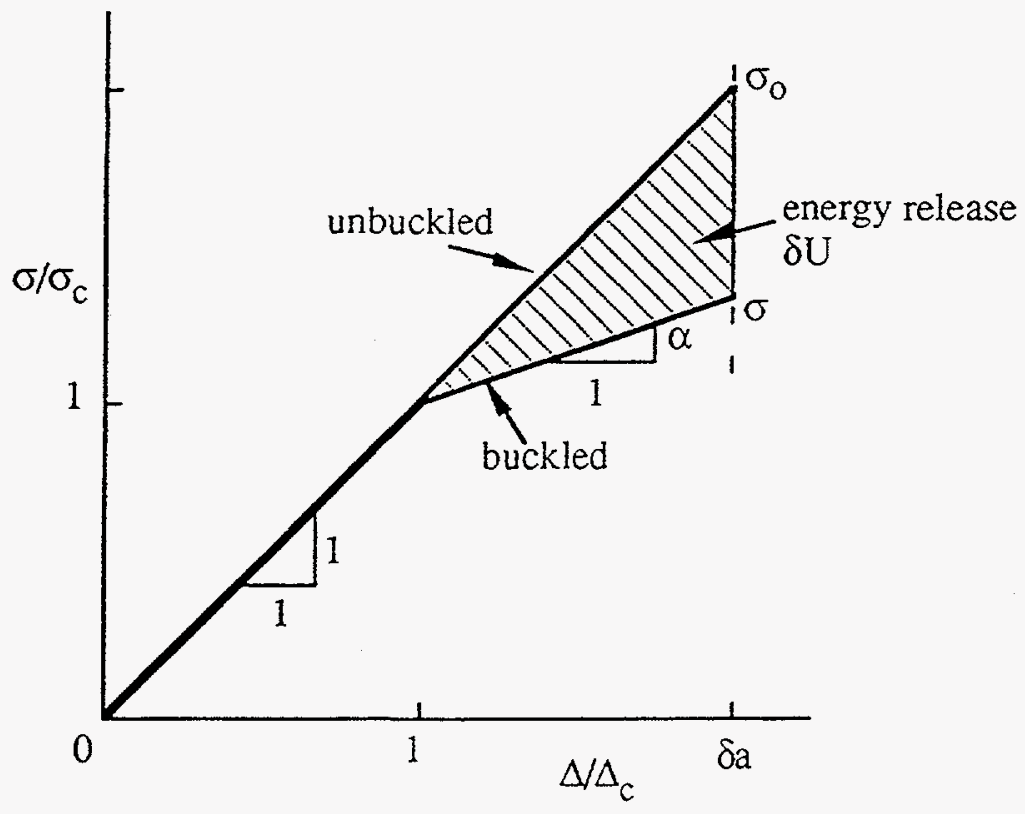

Figure 3.10. A plot of the relative edge stress as a function of displacement, for a clamped circular plate, showing the energy release, $\delta U$, that accompanies buckling.[53]

$$
G=\frac{(1-v)(1-\alpha)\left(\sigma_{o}^{2}-\sigma_{c}^{2}\right) t}{E}
$$

Stress distribution at the interface crack involves a combination of opening (Mode I) and shearing (Mode II). When the crack radius is much smaller than the film and the substrate is sufficiently thick, the stress intensity factors can be obtained directly from plain strain results. In the present work, such conditions are satisfied, and the effective value of stress intensity factor, $K$, can be defined as

$$
K^{2}=K_{I}^{2}+K_{I I}^{2}=\frac{E G}{\left(1-v^{2}\right)}
$$

Hence, eqn.(3.3.15) can be rewritten as 


$$
\frac{K}{\left(\sigma_{o} \sqrt{t}\right)}=\left[(1+v)(1-\alpha)\left\{1-\left(\frac{\sigma_{c}}{\sigma_{o}}\right)^{2}\right\}\right]^{1 / 2}
$$

The driving force for delamination at the remaining buckled sections can be derived from eqn.(3.3.11) and eqn.(3.3.17) as

$$
\frac{K}{\sigma_{o} \sqrt{t}}=\left[\left(\frac{1-\alpha}{1+v}\right)\left\{1-\frac{k E}{12 \sigma_{o}\left(1-v^{2}\right)}\left(\frac{t}{a}\right)^{2}\right)\right]^{1 / 2}
$$

Therefore, the driving force for crack propagation is a function of both the film thickness and the crack size. A plot of $K /\left(\sigma_{o} \sqrt{t}\right)$ vs. $(t / a)$ has a maximum value of $K, K_{\max }$, at a certain ratio of $t / a$. Therefore, if we let $A=\left[K /\left(\sigma_{o} \sqrt{t}\right)\right]^{2}$ and $X=t / a$, then $t=a X$ and an expression for $K_{\max }$ can be obtained from the condition $\partial A / \partial X=0$

$$
\begin{aligned}
& \frac{K_{\max }}{\sigma_{o} \sqrt{a}}=2\left(\frac{\sigma_{o}}{k E}\right)^{1 / 4}\left[\frac{(1-\alpha)(1-v)^{1 / 2}}{3(1+v)^{1 / 2}}\right]^{1 / 2} \\
& \text { when } X=2\left[\frac{\sigma_{o}\left(1-v^{2}\right)}{k E}\right]^{1 / 2}
\end{aligned}
$$

Equating $K_{\max }$ to the critical interface fracture toughness, $K_{i c}$, a critical initial separation radius, $a_{c}$, can be derived from eqn. (3.3.19) as

$$
a_{c}=\frac{3}{4(1-\alpha)}\left(\frac{K_{i c}}{\sigma_{o}}\right)^{2}\left[\frac{k E}{\sigma_{o}} \frac{(1+v)}{(1-v)}\right]^{1 / 2}
$$

Delamination will occur at initial separations with radii greater than $a_{c}$. Similarly, from eqn.(3.3.18), the critical film thickness, $t_{c}$, such that delamination occurs at $t>t_{c}$ is given by 


$$
t_{c}=2 a_{c}\left[\frac{\sigma_{o}\left(1-v^{2}\right)}{k E}\right]^{1 / 2}
$$

Inserting specific parameters for a-Si:H listed in Table III- 1 , and $K_{i c}=10^{5} \mathrm{~Pa} \cdot \mathrm{m}^{1 / 2}$, we obtain $a_{c} \approx 5 \mu \mathrm{m}$ and $t_{c} \approx 0.2 \mu \mathrm{m}$. This result shows that even the existence of a minute crack, or very small quantity of second phase at the interface can catastrophically accelerate the delamination of the film. However, it must be noted that the specific values of $a_{c}$ and $t_{c}$ are very sensitive to variation in the residual stress and in the adherence. 


\subsection{REDUCTION OF RESIDUAL STRESS}

\subsubsection{Introduction}

In section 3.3, it was shown that optimization of the deposition conditions and introduction of post-deposition steps helps to overcome some of the difficulties in making thick, high quality films. The requirements for a-Si:H charged particle detectors are: i) a high deposition rate, ii) residual stress, $\sigma \sim 100 \mathrm{MPa}$ or less, iii) an ionized dangling bond density $N_{d}{ }^{*} \sim 5 \times 10^{14} \mathrm{~cm}^{-3}$ or less, and iv) mobility-lifetime product $\mu \tau$ of charge carriers of the order of $10^{-8} \sim 10^{-7} \mathrm{~cm}^{2} / \mathrm{N}$.

The following knowledge from the previous section was a helpful guide to us on the way to fulfill the above mentioned requirements. The deposition rate was shown to increase when silane is diluted with helium. Helium-dilution led to an exceptionally low ionized dangling bond density and relatively poor electron mobility-lifetime product. Lowering the substrate temperature reduces the residual stress in films from values as high as $\sim 350 \mathrm{MPa}$ down to $\sim 100 \mathrm{MPa}$ or even smaller. The as-deposited material, however, has poor electronic quality. Also, it was shown that annealing at low temperatures can reduce the density of dangling bonds while the stress remains unchanged. Hence, the combination of the low-temperature growth, He-dilution and post-deposition heat treatment will lend a way to produce a material that has a low residual stress and electrical properties satisfactory for thick radiation detectors.

In this section, fabrication methods for thick a-Si:H films with low residual stress and satisfactory electronic properties are described. Also, the data revealing the structural changes appearing in the films under the new preparation conditions are presented. 


\subsubsection{Experimental Procedures}

Intrinsic layers with thicknesses of $5 \sim 50 \mu \mathrm{m}$ were prepared by the plasma enhanced chemical vapor deposition (PECVD) technique at an excitation frequency of 85 $\mathrm{MHz}$ from a gas mixture of $40 \% \mathrm{SiH}_{4}-60 \% \mathrm{He}$. The same gas mixture was also used for making thin $\mathrm{p}$ - and $\mathrm{n}$-doped layers for fabrication of $\mathrm{p}-\mathrm{i}-\mathrm{n}$ diodes. Corning 7059 glass coated with thin $(<200 \AA)$ indium tin oxide layer was used as a substrate. The substrate temperature was varied between $130^{\circ} \mathrm{C}$ and $250^{\circ} \mathrm{C}$ while all the other parameters were kept constant. Samples from pure silane were also deposited for comparison. Table III-6 summarizes the deposition conditions for He-diluted and undiluted layers. Post-deposition annealing at $160^{\circ} \mathrm{C}$ for 100 hours was applied to all samples.

The residual stress was determined by measuring the substrate curvature.(see section 2.3.2) The ionized dangling bond density, $N_{d}{ }^{*}$, before and after annealing, was measured by the hole onset technique described in section 3.1.2. The electron mobility, $\mu_{e}$, and mobility lifetime product, $(\mu \tau)_{\mathcal{e}}$, were measured by the standard time-of-flight technique. The vibrational spectra were detected by a FTIR Perkin-Elmer spectrometer in thresolution of $4 \mathrm{~cm}^{-1}$. The hydrogen desorption was measured with a home-made set up at a constant heating rate of $20^{\circ} \mathrm{K} / \mathrm{min}$. in the range between room temperature and $900^{\circ} \mathrm{C}$.

Table III-6 Deposition Parameters for Conventional and He-diluted Si films.

\begin{tabular}{|c|c|c|c|c|}
\hline Gas & Temp. $\left({ }^{\circ} \mathrm{C}\right)$ & $\begin{array}{c}\text { Power } \\
\text { Density } \\
\left(\mathrm{mW} / \mathrm{cm}^{2}\right)\end{array}$ & $\begin{array}{c}\text { Pressure } \\
(\mathrm{mT} \text { Torr })\end{array}$ & $\begin{array}{c}\text { Deposition } \\
\text { Rate } \\
(\mu \mathrm{m} / \mathrm{hr} .)\end{array}$ \\
\hline $100 \% \mathrm{SiH}_{4}$ & $\begin{array}{c}150,160,170, \\
190,250\end{array}$ & 40 & 300 & 2.3 \\
\hline $40 \% \mathrm{SiH}_{4}-60 \% \mathrm{He}$ & $\begin{array}{c}130,150,160, \\
170,190,250\end{array}$ & 90 & 500 & $3.5 \sim 4$ \\
\hline
\end{tabular}




\subsubsection{Optimization of the Deposition Conditions}

In Fig. 3.11 and Fig. 3.12, the changes in stress and dangling bond density as a function of the deposition temperature, before and after the heat treatment, are plotted for He-diluted, and for standard a-Si:H samples. For both the He-diluted and standard samples, one clearly observes a pronounced anti-correlation between the stress and the $N_{d}{ }^{*}$. It has been reported that when the substrate temperature is lowered, a microvoid-rich structure is formed, which is capable of releasing the stress.[21] These microvoids provide spaces for the compressively stressed a-Si:H network to relax, but introduce additional defect states at the same time.

Both the $N_{d}{ }^{*}$ and the stress experience strong gradients between the substrate temperature of $150^{\circ} \mathrm{C}$ and $170^{\circ} \mathrm{C}$. This sharp change in both parameters can be attributed to the increased content of $\mathrm{SiH}_{2}$ bond configuration below $170^{\circ} \mathrm{C}$ as discussed in section 3.3. and agrees well with other works. $[56,57]$ The post-annealing step affects significantly the $N_{d}{ }^{*}$ while the stress remains unchanged. Annealing at $160^{\circ} \mathrm{C}$ seemed to rearrange the interstitial hydrogen atoms so that they passivate dangling bonds. The annealing effect on

the $N_{d}{ }^{*}$ is more pronounced in the low temperature materials than in the high temperature ones. From these plots, deposition temperature of $150^{\circ} \mathrm{C}$ was chosen as an optimum value, because it produced films of low enough stress $(\sim 100 \mathrm{MPa})$ and $N_{d}{ }^{*}\left(\sim 7 \times 10^{14}\right.$ $\mathrm{cm}^{-3}$ ) after annealing. Films deposited at $160^{\circ} \mathrm{C}$ also showed good values of residual stress and $N_{d}{ }^{*}$ on the average, but had large sample-to-sample variation since they are in the middle of the abrupt transition. 


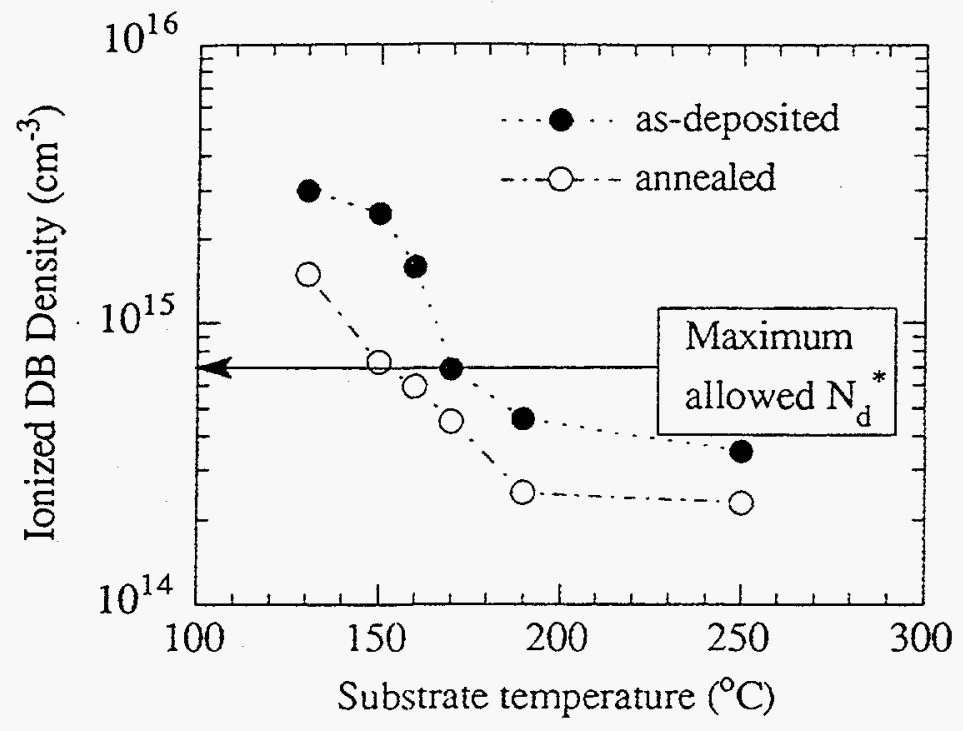

(a)

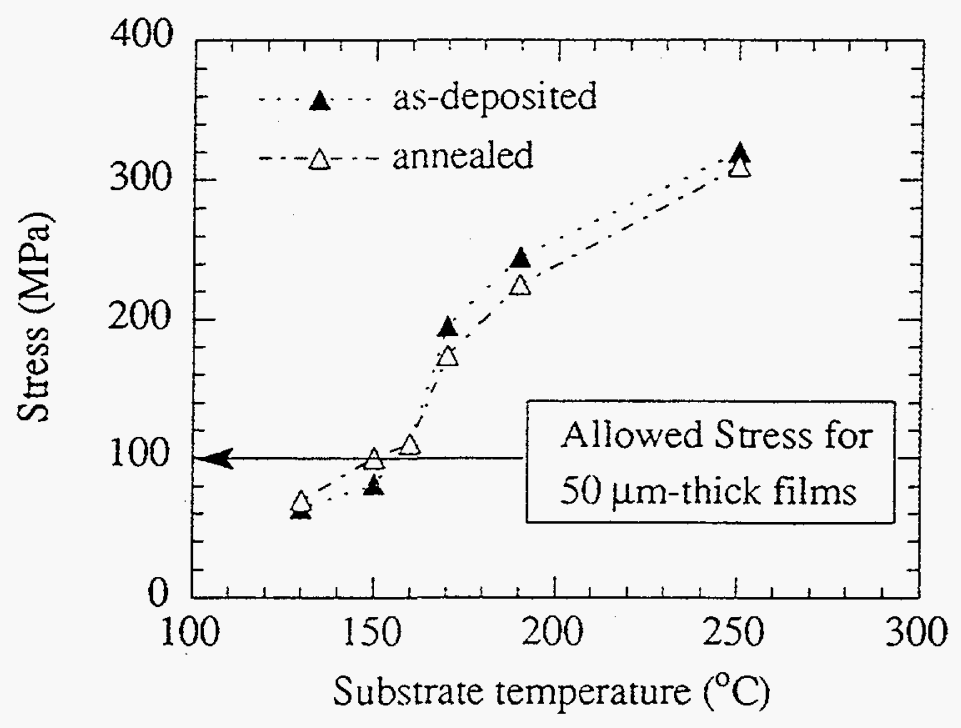

(b)

Figure 3.11. Change in (a) ionized dangling bond density, $N_{d}{ }^{*}$, and (b) residual stress with deposition temperature for He-diluted samples. Closed and open symbols represent as-deposited and annealed samples, respectively. 


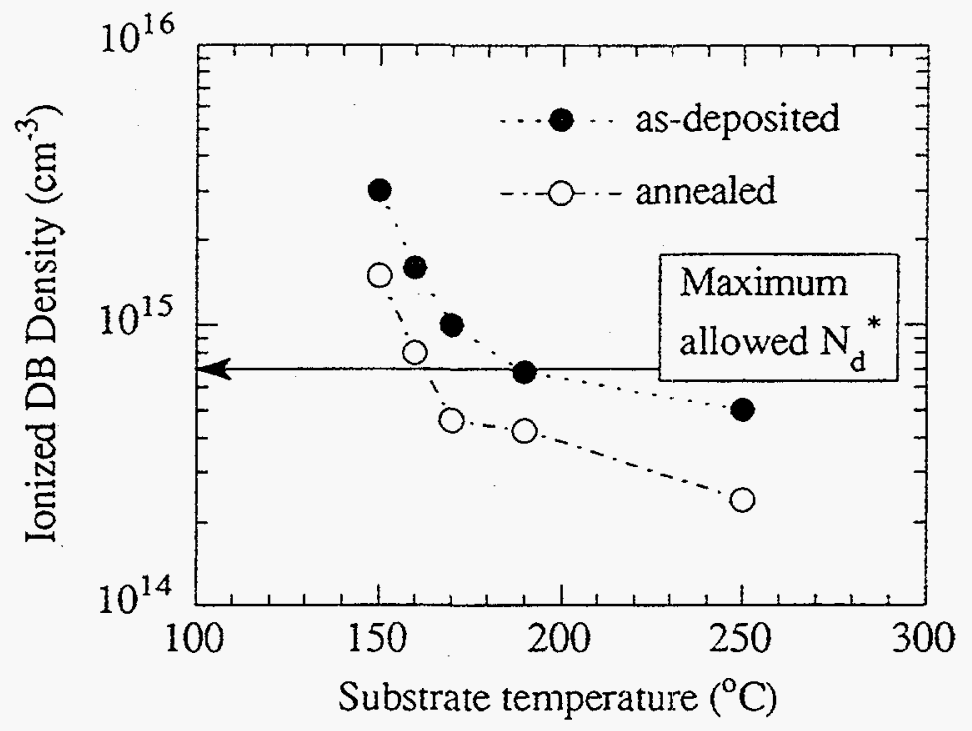

(a)

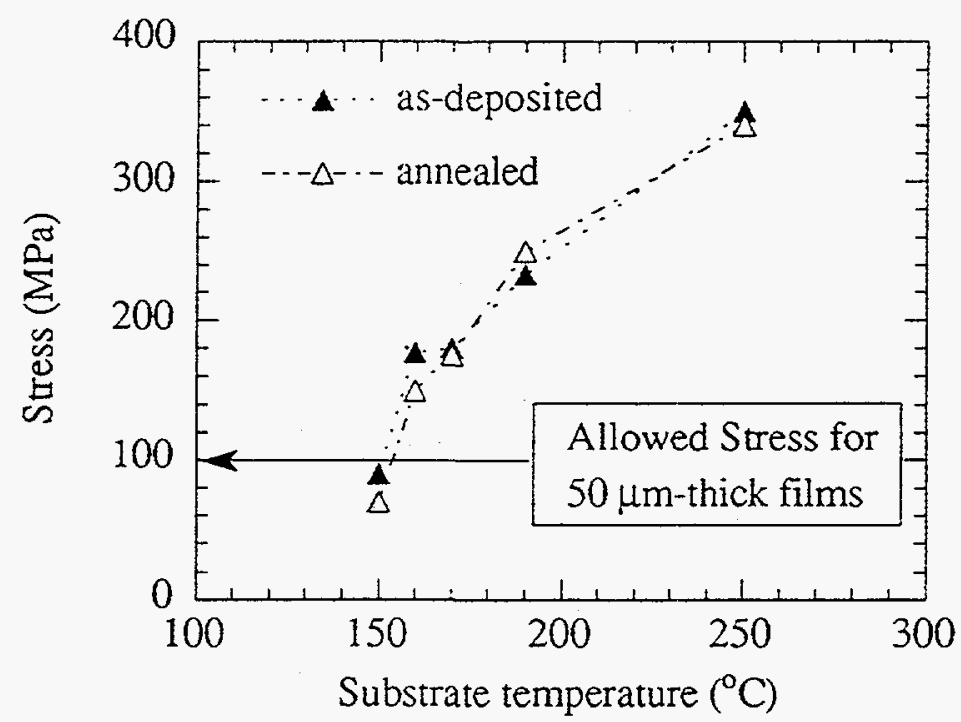

(b)

Figure 3.12. Change in (a) ionized dangling bond density, $N_{d}{ }^{*}$, and (b) residual stress with deposition temperature for standard samples. Closed and open symbols represent as-deposited and annealed samples, respectively. 


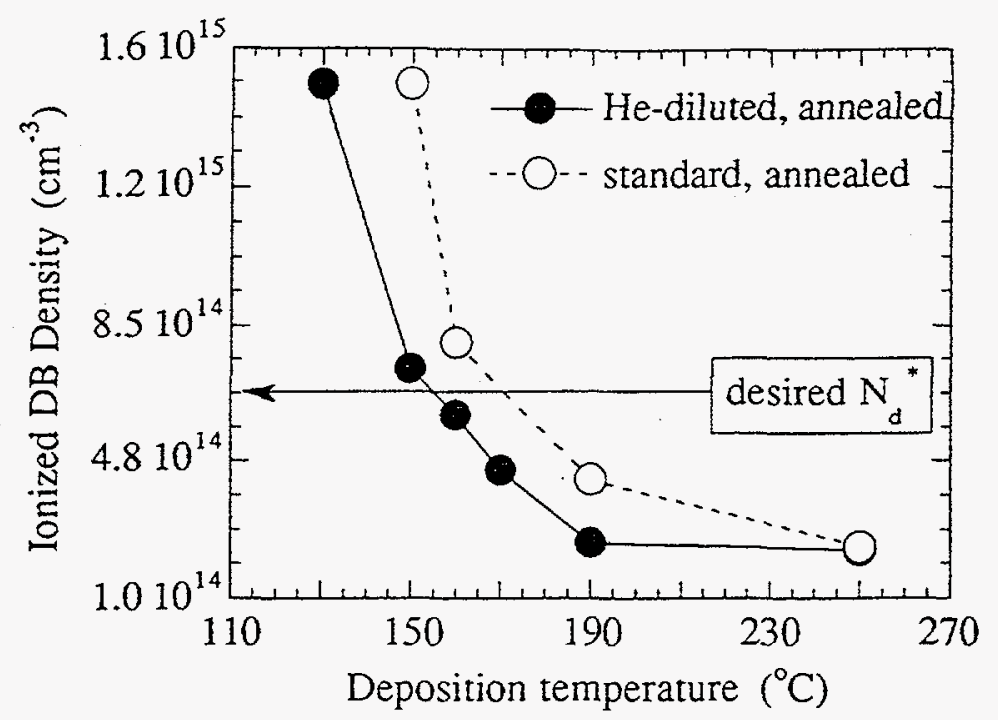

Figure 3.13. Change in ionized dangling bond density with deposition temperature for annealed samples

In figures 3.11 and 3.12 the desired values of the $N_{d}{ }^{*}$ and the stress for detector application are given by arrows. For the as-prepared samples, it becomes clear from the figure that no deposition temperature provides films satisfying the desired stress and $N_{d}{ }^{*}$ at the same time. For standard samples, annealing improves the situation significantly, but at the cross-over point around $160^{\circ} \mathrm{C}$ the stress value is still higher than the desired value. The He-diluted and post-annealed samples show further quantitative improvement. This is demonstrated in Fig. 3.13.

Table MI-7 lists the results of all four important electronic parameters for samples prepared at $250^{\circ} \mathrm{C}$ and $150^{\circ} \mathrm{C}$ with and without He-dilution, before and after annealing. Only the material prepared with He-dilution at $150^{\circ} \mathrm{C}$ and post-annealed for 100 hours provides a satisfactory quality for realization of particle detectors.

The electron drift mobility, $\mu_{e}$, seemed to decrease slightly with decreasing deposition temperature, but it is still within the range of experimental error. The $\mu_{e}$ did not 
change with annealing. The electron mobility-lifetime product, $(\mu \tau)_{e}$, of standard materials is about a factor of three higher than that of the He-diluted ones for the samples deposited at $250^{\circ} \mathrm{C}$, and the trend is reversed for those deposited at $150^{\circ} \mathrm{C}$. The $\mu \tau$ increased by a factor of two with annealing, which is thought to be partially due to the decreased deep trap (dangling bond) densities after annealing. The $\mu \tau$ value of $2 \times 10^{-8} \mathrm{~cm}^{2} / \mathrm{V}$ for the Hediluted, low temperature $\left(150^{\circ} \mathrm{C}\right)$ deposited and annealed sample corresponds to a mean free path of $60 \mu \mathrm{m}$ under an electric field of $30 \mathrm{~V} / \mu \mathrm{m}$, which is a normal bias field for a$\mathrm{Si}: \mathrm{H} \mathrm{p}-\mathrm{i}-\mathrm{n}$ detectors, and is sufficient for thick detectors.

This result implies that the low temperature deposition only lowers the internal stress and the He-dilution facilitates the restoration of low defect densities.

Table III-7. Measured Electron Transport Parameters and Stress

\begin{tabular}{|c|c|c|c|c|}
\hline Samples & $\begin{array}{c}\mu_{\mathrm{c}} \\
\left(\mathrm{cm}^{2} / \mathrm{V} \cdot \mathrm{s}\right)\end{array}$ & $\begin{array}{c}(\mu \tau)_{e} \\
\left(\mathrm{~cm}^{2} / \mathrm{V}\right)\end{array}$ & $\mathrm{N}_{\mathrm{d}}{ }^{*}\left(\mathrm{~cm}^{-3}\right)$ & $\begin{array}{l}\text { Stress } \\
(\mathrm{MPa})\end{array}$ \\
\hline \multicolumn{5}{|c|}{ Standard (deposited at $250^{\circ} \mathrm{C}$ ) } \\
\hline as-deposited & 1.2 & $1.2 \times 10^{-7}$ & $6 \times 10^{14}$ & 350 \\
\hline annealed & 1.2 & & $3 \times 10^{14}$ & 330 \\
\hline \multicolumn{5}{|c|}{ Standard (deposited at $150^{\circ} \mathrm{C}$ ) } \\
\hline as deposited & 0.5 & $3 \times 10^{-9}$ & $2.5 \times 10^{15}$ & 90 \\
\hline annealed & 0.5 & $7 \times 10^{-9}$ & $1.5 \times 10^{15}$ & 70 \\
\hline \multicolumn{5}{|c|}{ He-diluted(deposited at $250^{\circ} \mathrm{C}$ ) } \\
\hline as-deposited & 1.2 & $3 \times 10^{-8}$ & $3 \times 10^{14}$ & 320 \\
\hline annealed & 1.2 & & $3 \times 10^{14}$ & 310 \\
\hline \multicolumn{5}{|c|}{ He-diluted(deposited at $150^{\circ} \mathrm{C}$ ) } \\
\hline as-deposited & 0.8 & $1 \times 10^{-8}$ & $3 \times 10^{15}$ & 70 \\
\hline annealed & 0.8 & $2 \times 10^{-8}$ & $7 \times 10^{14}$ & 80 \\
\hline
\end{tabular}




\subsubsection{IR Absorption and Raman Scattering}

Because of the disorder in an a-Si:H network, the phonon spectrum of a-Si:H is a little different from that of crystalline silicon. The phonon density of states is broadened due to the deviation in bond length, and additional phonon modes are introduced by the hydrogen bonding. There are also excess low frequency modes which are associated with disordered materials. Therefore, observing the phonon distribution in the material provides valuable information about the bonding structure.

The experimental techniques commonly used to measure phonon distribution are IR absorption, Raman scattering and neutron scattering.[58] The behavior of molecules is modeled by a harmonic oscillator. When electromagnetic radiation is incident on a molecule it undergoes a vibrational, rotational or electronic transition. Only the vibrational modes will be taken into account in this study because rotational modes cannot be observed in a rigid network and the infrared radiation is not intense enough to cause an electronic transition.

In Raman scattering, a charged particle such as an electron will generate an electromagnetic wave when it vibrates.[59] The frequency of the wave will match that of the vibrating charge. The electric field associated with an electrically neutral particle will be proportional to its polarization. Since the electric field generated by a vibrating molecule is proportional to the polarization, three different waves will be scattered. The first has a frequency which matches that of the incident radiation. The other two waves have frequencies that are shifted from that of the incident radiation. These are inelastically scattered, and are referred to as Raman scattered radiation. The magnitude of the shift in frequency of the Raman scattered radiation corresponds to the vibrational frequency of the scattering molecule. Thus, the Raman spectrum can serve as a fingerprint for identifying unknown substances. In order to have Raman scattered radiation, there needs to be at least one component of the polarizability tensor whose derivative with respect to the normal 
coordinate of the molecule has a non-zero value. [60] The scattering intensity, $I(\omega)$, as a function of the phonon frequency, $\omega$, is

$$
I(\omega)=\frac{C(\omega)\lfloor 1+n(\omega)\rfloor g(\omega)}{\omega}
$$

where $C(\omega)$ is the coupling constant, $n(\omega)$ is the phonon occupancy factor and $g(\omega)$ is the phonon density of states. $C$ is roughly proportional to $\omega^{2}$ for low frequencies, so that the Raman intensity is related to the phonon density of states by

$$
g(\omega)=\text { Constant } \frac{I(\omega)}{\omega[1+n(\omega)]}
$$

In IR absorption, the incident photon couples to the dipole moment induced by the phonon vibration. For the vibrational modes to be IR active, the radiation must cause a change in the electric dipole moment of the molecule and the transition must be from one quantum vibrational state to a second which is immediately adjacent in energy.[61] Hence, Infrared activity requires that (i) the dipole moment of the molecule must change with atomic displacement and (ii) the expectation value of a normal coordinate in its ground state must be non-zero. The absorption spectrum, $\alpha(\omega)$, is given by

$$
n \alpha(\omega)=\pi^{2}\left(4 \pi N e^{2} / M\right) f(\omega) g(\omega)
$$

where $f(w)$ is the oscillator strength, $N$ is the density of oscillators, $M$ is their mass, and $n$ is the refractive index.

Since the polarizability of a molecule is in part a function of its volume, any vibrational activity which changes the volume of the molecule may satisfy the Ramanactivity criterion. In general, for molecules having a center of symmetry, the modes that are IR active are Raman inactive and vice versa. Hence, the Raman and IR spectra of a scattering medium possessing a center of symmetry will be complementary. 
Table III-8. The set of Si-H vibrational modes for $\mathrm{SiH}, \mathrm{SiH}_{2}, \mathrm{SiH}_{3}$ groups. The frequencies in parenthesis are estimates. The frequencies in parenthesis are estimates. I, R, and $\mathrm{N}$ in the activity column indicate IRactive, Raman-active and inactive modes, respectively.[64]

\begin{tabular}{cccc}
\hline Frequency $\left(\mathrm{cm}^{-1}\right)$ & Bonding configuration & Activity & Vibration mode \\
\hline 2140 & $-\mathrm{Si}=\mathrm{H}_{3}$ & $\mathrm{I}, \mathrm{R}$ & stretch \\
2090 & $=\mathrm{Si}=\mathrm{H}_{2}$ & $\mathrm{I}, \mathrm{R}$ & stretch \\
2000 & $\equiv \mathrm{Si}-\mathrm{H}$ & $\mathrm{I}, \mathrm{R}$ & stretch \\
907 & $-\mathrm{Si} \equiv \mathrm{H}_{3}$ & $\mathrm{I}, \mathrm{R}$ & deformation (degenerate) \\
880 & $=\mathrm{Si}=\mathrm{H}_{2}$ & $\mathrm{I}, \mathrm{R}$ & scissors \\
862 & $-\mathrm{Si} \equiv \mathrm{H}_{3}$ & $\mathrm{I}, \mathrm{R}$ & deformation (symmetric) \\
850 & $=\mathrm{Si}=\mathrm{H}_{2}$ & $\mathrm{I}, \mathrm{R}$ & wag \\
$(820)$ & $=\mathrm{Si}=\mathrm{H}_{2}$ & $\mathrm{R}$ & twist \\
630 & $\equiv \mathrm{Si}-\mathrm{H}_{1}$ & $\mathrm{I}$ & bend (rock or wag) \\
& $=\mathrm{Si}=\mathrm{H}_{2}$ & $\mathrm{I}, \mathrm{R}$ & rock \\
& $-\mathrm{Si} \equiv \mathrm{H}_{3}$ & $\mathrm{I}, \mathrm{R}$ & rock, wag \\
$(500)$ & $-\mathrm{Si}=\mathrm{H}_{3}$ & $\mathrm{~N}$ & twist \\
\hline
\end{tabular}

The IR and Raman spectra of crystalline silicon reflect the selection rules due to the symmetry in the lattice for optical transitions and are very different from the phonon density of states. The momentum selection rules are relaxed in the amorphous material so that all the phonons contribute to the spectrum, enabling us to estimate the density of specific bonds from a spectrum.[62] The relaxation of the momentum selection rules is easily seen in the Raman spectrum in the vicinity of the transverse optical (TO) modes near the wavenumber $500 \mathrm{~cm}^{-1}$. The crystalline Si peak at $520 \mathrm{~cm}^{-1}$ is replaced by a broad band at $480 \mathrm{~cm}^{-1}$ in the amorphous film.[63] The shift is due to the loss of $k$-conservation, so that 
the peak is an average over the TO band. The disorder relaxes the selection rules and mixes in more of the TO band, broadening it and moving it to lower frequency. Thus, the degree of disorder in a-Si:H can be estimated by measuring the TO band peak position, $\omega_{\mathrm{TO}}$ and its full-width-half-maximum (FWHM), $\Delta \omega$.

The hydrogen bonding is very sensitive to the deposition conditions and its diffusion is associated with the metastable changes of the electronic properties. Hence, characterization of hydrogen bonding structure is essential to study the relationship between microstructure and process parameters in a-Si:H. Since hydrogen is a light atom, vibration is almost entirely confined to it, and the phonon frequency of the hydrogen modes are above the silicon network modes, making them easy to observe. Table III- 8 summarizes the normal modes of various Si-H bonding configurations.[64]

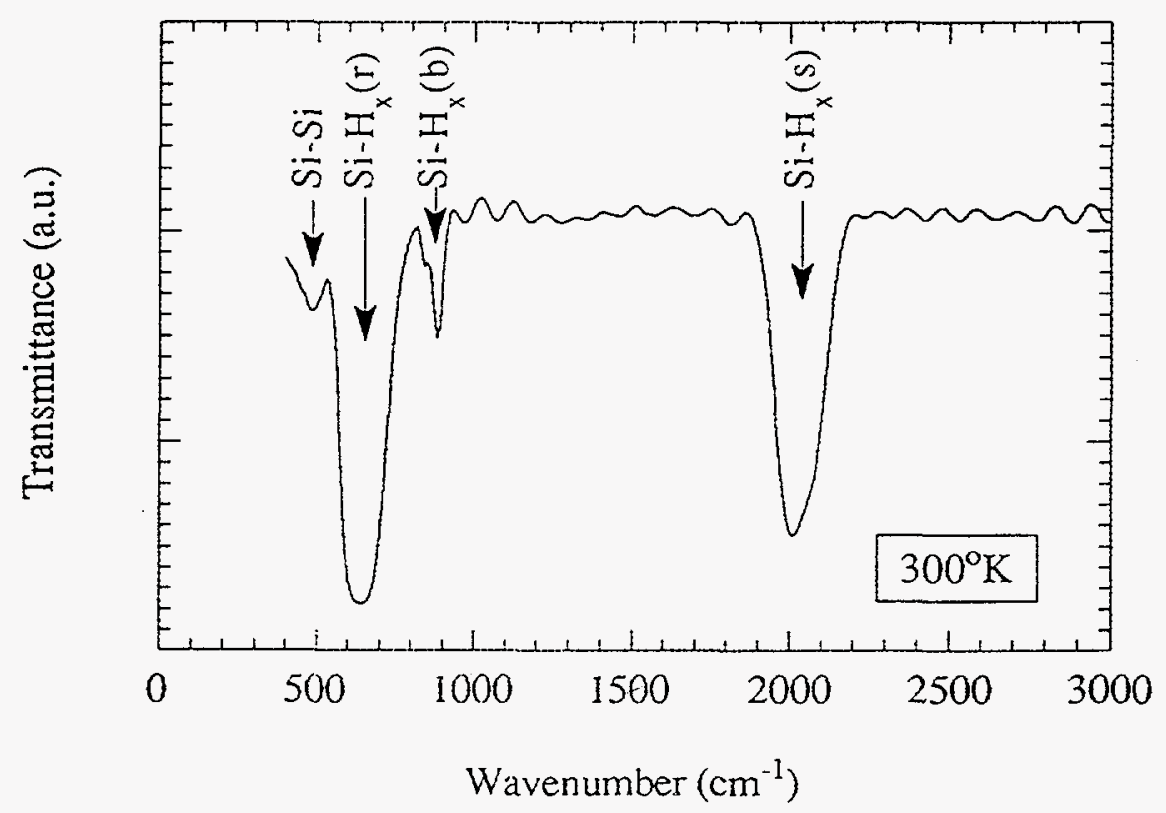

Figure 3.14. FTIR transmittance spectrum of the He-diluted, lowtemperature $\left(150^{\circ} \mathrm{C}\right)$ deposited and post-annealed sample. 


\subsubsection{Microstructures of the Conventional a-Si:H and the New Films}

In Fig. 3.14, a typical Fourier transfromed infrared (FTIR) transmittance spectrum of the various films is depicted. In addition to the stretching (s) band around $2000 \mathrm{~cm}^{-1}$ and the rocking ( $\mathrm{r}$ ) band at $\sim 660 \mathrm{~cm}^{-1}$ which have been reported for high quality a$\mathrm{Si}: \mathrm{H},[63]$ one clearly sees a doublet of modes around $850 \mathrm{~cm}^{-1}$ which correspond to the Si$\mathrm{H}_{2}$ and $\mathrm{Si}-\mathrm{H}_{3}$ bending (b) and wagging (w) region. Also, there is an additional band at $\sim 500 \mathrm{~cm}^{-1}$, which can be assigned to $\mathrm{H}$-induced $\mathrm{Si}-\mathrm{Si}$ vibrations.

Figure 3.15 shows an expanded plot of the $\mathrm{Si}-\mathrm{H}_{\mathrm{X}}$ stretching (Fig. 3.15(a)), $\mathrm{Si}-\mathrm{H}_{\mathrm{X}}$ bending (Fig. 3.15(b)) and the Si-Si:H (Fig. 3.15(c)) regions. Figure 3.15(a) allows us to resolve the two peaks in the stretching region. The first broad peak around $2000 \mathrm{~cm}^{-1}$ is known to be due to vibration of the $\mathrm{Si}-\mathrm{H}_{1}$ bond in the interior of the amorphous network. [65] The second one is shifted to higher wavenumber by $\sim 80 \mathrm{~cm}^{-1}$. Such a shift is typical for the $\mathrm{SiH}_{\mathrm{x}}$ vibration on the $\mathrm{Si}$ surface, as known from studies of $\mathrm{H}$-terminated c-Si monosurfaces.[11]. The appearance of this mode is a proof for the existence of voids in our materials, because this mode originates from the inner surfaces of microvoids terminated by hydrogen. The microvoids are responsible for the low stress and also for low $\mu \tau$ values. On one hand, they provide spaces for relaxing local strains of the network, and on the other hand they act as traps for charge carriers in addition to the dangling bonds. It is also noted from the data in Table III-3 of section 3.3.1 and in Table III-7 of section 3.4.3 that the relationship $\mu \tau N_{d}{ }^{*}=$ Const. does not hold for He-diluted materials. This is probably because the microvoids and related dangling bonds introduce complexity to the transport property and the $N_{d}{ }^{*}$ may not be one third of the $N_{d}$ any more. However, further work is required to draw any concrete explanation. 


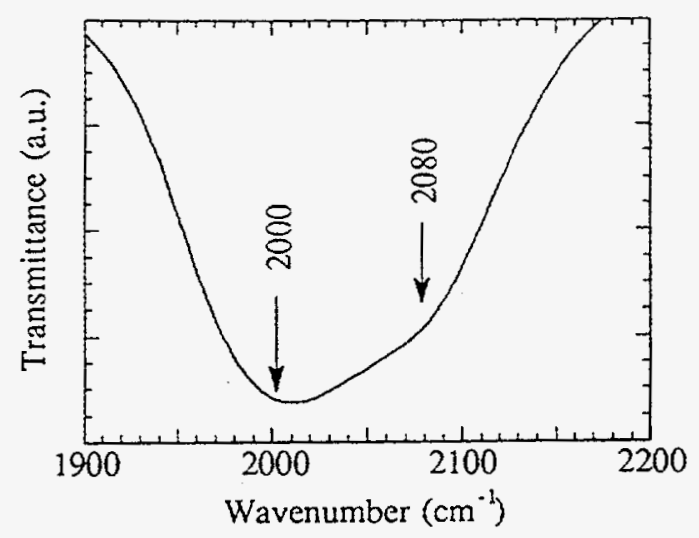

(a)

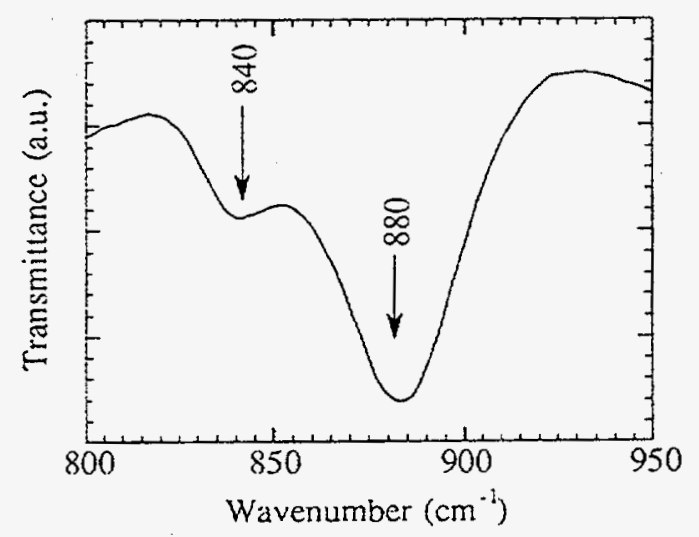

(b)

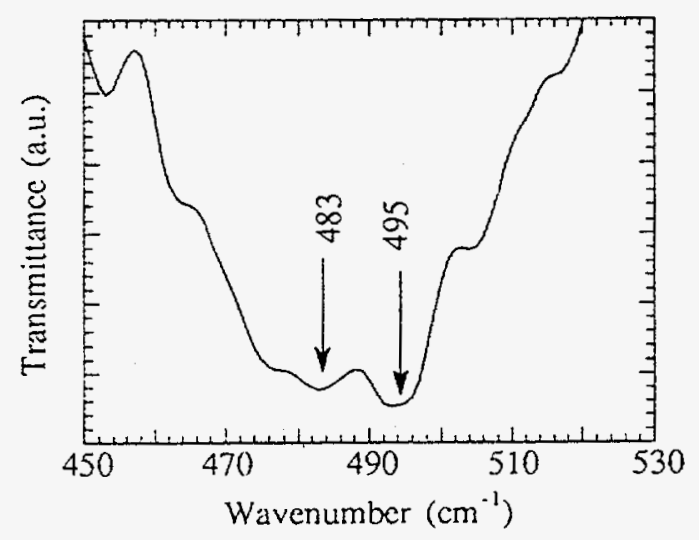

(c)

Figure 3.15. Expanded FTIR spectra for the (a) stretching, (b) bending, and (c) Si-Si vibration region 
The considerable concentration of $\mathrm{Si}-\mathrm{H}_{2}$ bonds in a void-rich network is in agreement with the observation reported previously in the literature on low-temperature deposited a-Si:H films.[21] A-Si:H has a well-defined two-phase inhomogeneous hydrogen bonding structure in which hydrogenated voids are embedded in an amorphous matrix with a lower hydrogen concentration. The void surface comprises either $\mathrm{Si}-\mathrm{H}$ or $\mathrm{Si}$ $\mathrm{H}_{2}$ bonding configurations and the density of voids varies with the deposition conditions. The appearance of the scissor mode around $880 \mathrm{~cm}^{-1}$ in Fig. 3.15(b) provides the information that our film contains a non-negligible amount of $\mathrm{Si}-\mathrm{H}_{2}$ bonds. The peak at $840 \mathrm{~cm}^{-1}$ is due to the existence of short $\left(\mathrm{Si}-\mathrm{H}_{2}\right)_{\mathrm{n}}$ chains in the network on the void surfaces.

The appearance of the mode near $500 \mathrm{~cm}^{-1}$ is a characteristic of the He-diluted films. It is not observed in the conventional a-Si:H. This is an inactive mode and not a mode of an $\mathrm{Si}-\mathrm{SiH}_{3}$ molecular cluster. Instead, the symmetry assignment was derived from a consideration of a larger cluster $\mathrm{Si}_{4}-\mathrm{SiH}_{3}$. [64] This spectrum is different from the typical Raman spectra reported for a-Si:H.(see section 3.4.4) In the Raman spectrum, the transverse optical mode appears at $520 \mathrm{~cm}^{-1}$ for crystalline $\mathrm{Si}$ and a broad band appears at $480 \mathrm{~cm}^{-1}$ for amorphous silicon. There is a pronounced shoulder of this band in the region between 480 and $520 \mathrm{~cm}^{-1}$, which can be caused by the existence of tiny crystalline inclusion, covered by $\mathrm{H}$, in the film. In this case the $\mathrm{Si}-\mathrm{Si}$ transverse optical (TO) vibration can be induced by $\mathrm{H}$ and becomes observable in the IR-spectrum. As helium has a thermal conductivity comparable to that of hydrogen[66], adding He to the plasma is believed to promote ordering of the atoms by transferring thermal energy to them. These clusters could not be observed by a conventional (100 kV) TEM, and therefore are believed to be only a few angstroms in size. However, they are considered to be responsible for the relatively low density of ionized dangling bonds in the He-diluted material despite the low $\mu \tau$ values. 
The IR spectra are the keys for explaining how the deposition conditions influence the microstructure of the deposited film. The CVD growth conditions of high temperature and undiluted silane result in the predominance of $\mathrm{Si}-\mathrm{H}$ bonds.[64] On the other hand the PVD conditions of low temperature, which give a void-rich morphology, are associated with large concentrations of $\mathrm{Si}-\mathrm{H}_{2}$ bonds. The void-rich structure is desirable for a low residual stress, yet undesirable for good electrical property. The voids are also responsible for the low $\mu \tau$ values since they act as traps for charge carriers. However, as discussed in section 3.3 .2 , annealing at $160^{\circ} \mathrm{C}$ restores the electronic quality without affecting the stress.

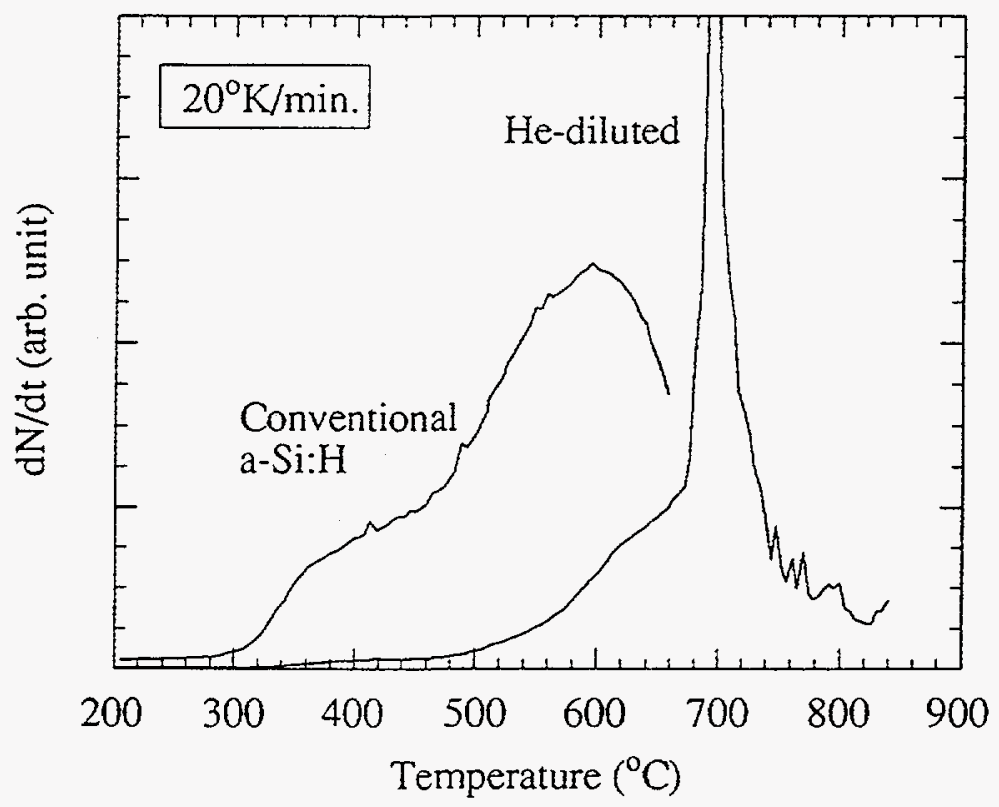

Figure 3.16. Hydrogen effusion spectrum of the conventional a-Si:H and of the He-diluted and low-temperature deposited sample. 
Figure 3.16 shows a typical hydrogen-effusion spectrum for the new samples. For comparison, also an effusion spectrum of a conventional a-Si:H is shown. The high thermal stability of the He-diluted material seen by a strong $\mathrm{H}$-evolution peak at $\sim 700^{\circ} \mathrm{C}$ is surprising. Usually, one expects the $\mathrm{Si}-\mathrm{H}_{2}$ bonds to decompose at $\sim 400^{\circ} \mathrm{C}[67]$ More experiments need to be done to understand the unusual $\mathrm{H}$ desorption kinetics in this material. 


\subsection{SUSBtrate Pattering For InCREASEd MEChanical STABILITY}

An alternative approach to avoid degrading the electronic quality of the a-Si:H by changing the deposition conditions is to control the substrate morphology. By patterning the substrate, space for strain relaxation can be provided, or the net stress imposed on the substrate can be lowered.[68,69] Furthermore, by dividing the film into 'pixels', we can make a 2-dimensional array for a position sensitive detector.

\subsubsection{Square Island Structure}

The first attempt was to pattern the substrate into small square islands by making grooves chemically or mechanically. This selective area growth helps reduce the stress developing in the film by decreasing the contact area which serves as a constraint and by providing spaces to relax the strain. The selective area growth is reported to reduce the thermally induced residual stress in GaAs/Si heteroepitaxy system according to the following equation $[68,70]$

$$
\sigma=\sigma_{o}[1-\exp \{-k(w-x)\}]
$$

where $\sigma$ and $\sigma_{o}$ are residual stress for selective area growth and normal growth, respectively, $w$ is half the patterned width, $x$ is the distance from the center of the patterned film, and $k$ is the interfacial compliance parameter given by:

$$
\begin{aligned}
& k^{2}=\frac{1}{k_{o}}\left[\frac{1-v_{f}^{2}}{E_{f} t_{f}}+\frac{1-v_{s}^{2}}{E_{s} t_{s}}+\frac{3\left(1-v_{f}^{2}\right)\left(1-v_{s}^{2}\right)\left(t_{f}+t_{s}\right)^{2}}{E_{f} t_{f}^{3}\left(1-v_{s}^{2}\right)+E_{s} t_{s}^{3}\left(1-v_{f}^{2}\right)}\right] \\
& \text { and } k_{o}=\frac{2}{3}\left[\frac{\left(1+v_{f}\right) t_{f}}{E_{f}}+\frac{\left(1+v_{s}\right) t_{s}}{E_{s}}\right]
\end{aligned}
$$


The above equation implies that the stress in the film becomes maximum at the center $(x=0)$ and drops exponentially near the edge $(x=w)$ of the patterned mesas. Therefore, if the patterned width is small enough to offset the large $k$, the maximum stress in the film will be decreased by $\exp (-k w)$. In Fig. 3.17, a schematic diagram and stress profile of the normal growth and selective area growth.
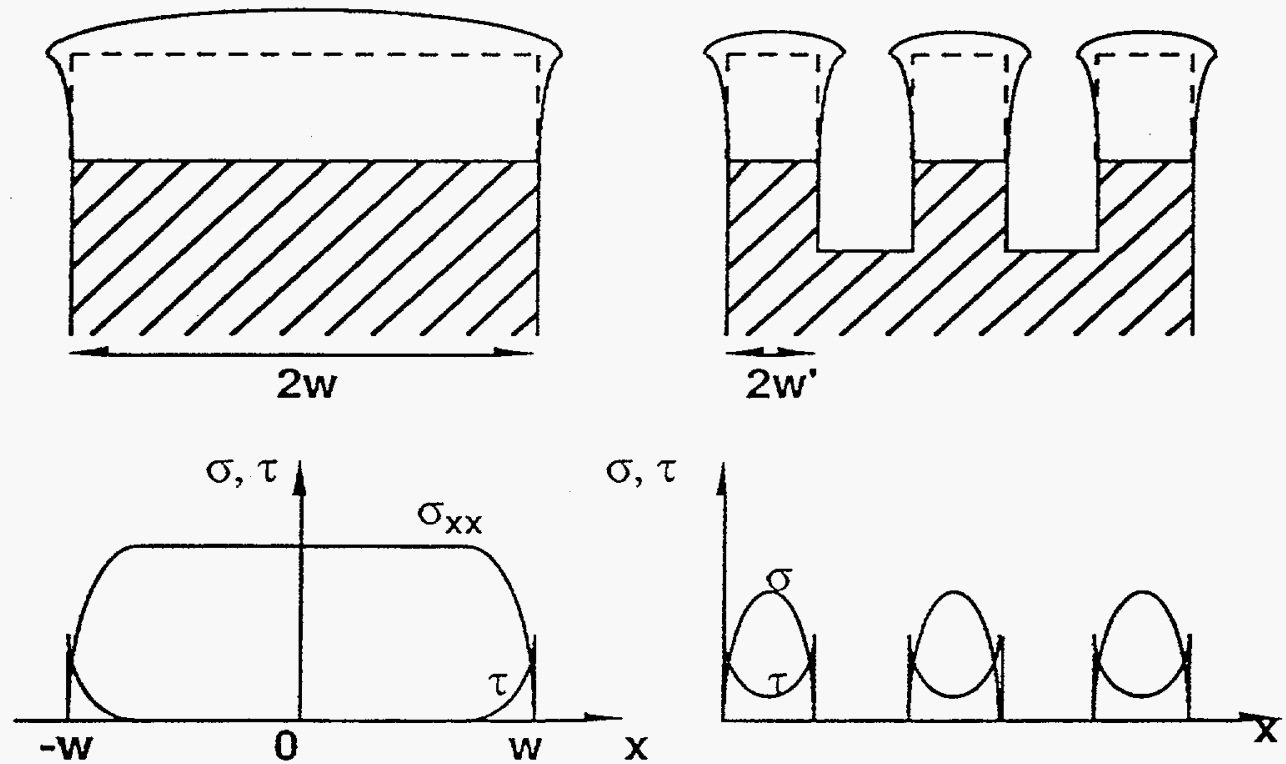

Figure 3.17. Schematic diagram and cross-sectional stress profile of normal growth and of selective area growth. In the figures at the top, the dotted-line and the solid line represent shapes of the film under a uniform compression and after the strain is released, respectively. In the figures at the bottom, $\sigma$ and $\tau$ represent normal and shear component of the stress, respectively. $\sigma_{x x}$ stands for the stress acting on the cross section of the film in the lateral direction 


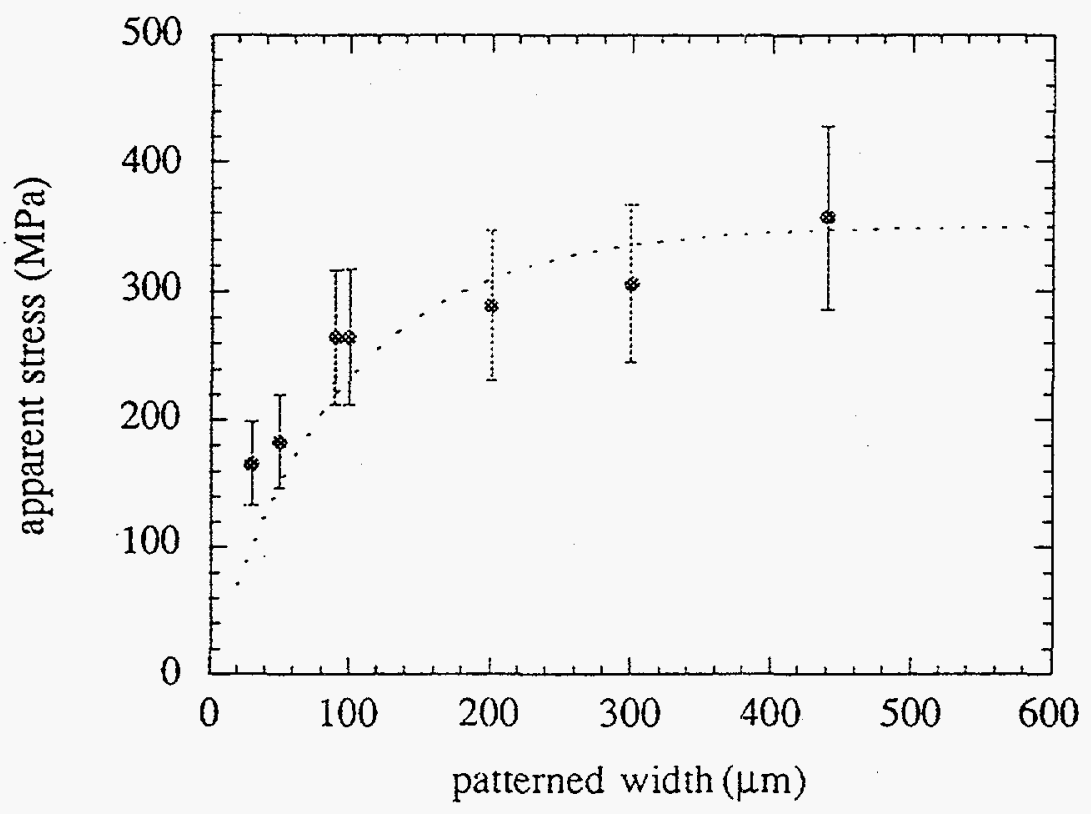

Figure 3.18. Comparison of calculated and experimental results for stress reduction in the a-Si:H films grown on square islands as a function of the lateral dimension of the squares. The dashed line and closed circles represent calculated and experimental data, respectively.

However, since the thickness and the stress distribution for patterned substrates are not uniform across the substrate, eqn. (2.3.6) in section 2.3 .2 cannot be used to estimate the stress in the patterned substrate. Also, since the films are amorphous, other stress measurement techniques, such as photoluminescence or X-ray diffraction, cannot be applied. Therefore, we used an equivalent thickness, $t_{s,}$ eqv, which is a thickness of an imaginary, plain substrate having the same volume and area of the patterned substrate, in place of the substrate thickness in eqn. (2.3.6). The resulting "apparent stress", is the stress corresponding to a curvature of a plain substrate of thickness $t_{s, \text { eqv }}$ and can be represented as follows: 


$$
\sigma_{a p p}=\frac{E_{s}}{6\left(1-v_{s}\right)} \frac{t_{s, e q v}^{2}}{t_{f}} \frac{1}{R}
$$

A single crystalline Si wafer coated with a thin $(\sim 500 \AA)$ layer of $\mathrm{Cr}$ was chosen as a substrate because of good adhesion, surface uniformity, and convenience for further processing. The thin $\mathrm{Cr}$ layer is known to improve adhesion of a-Si:H film to the substrate without changing overall stress state significantly, and forms a quasi-ohmic contact with both $\mathrm{n}$ - and p-type layers.[26] Trenches of $80 \mu \mathrm{m}$ in width and $100 \mu \mathrm{m}$ in depth were cut on the surface of $\mathrm{Si}$ wafers using a diamond saw to make square mesas of lateral dimensions ranging from $40 \mu \mathrm{m}$ to $440 \mu \mathrm{m}$. The patterned substrate was then sputter coated with $\mathrm{Cr}$, and a-Si:H films of up to $32 \mu \mathrm{m}$ in thickness were deposited. The deposition parameters were chosen from Fig. 3.2 in section 3.1 .5 such that a dangling bond density as low as possible $\left(N_{d}^{*}=5 \times 10^{14} \mathrm{~cm}^{-3}\right)$ was obtained. For comparison, films were also grown on flat surfaces. The substrate curvature was measured by scanning the backside of the wafer with an Alphastep 200 Profilometer. The fractured cross section was observed by scanning electron microscopy (SEM).

Figure 3.18 shows the stress values estimated from the substrate curvature and theoretical values calculated from eqn. (3.5.4). Theoretical values calculated with eqn. (3.5.1) (3.5.3) are indicated by a dashed line. The change in equivalent thickness with the patterned width were compensated in the calculation. The measured and calculated values agree well with each other within the experimental error. The apparent stress decreased exponentially as the lateral dimension of the mesas decreased. Approximately $40 \%$ stress reduction was achieved when the patterned width was decreased to $40 \mu \mathrm{m}$. However, such small patterns are not practical because the trench area is larger than the active area. Also, the stress analysis can no more be approximated as 2-dimensional and is more complicated. 


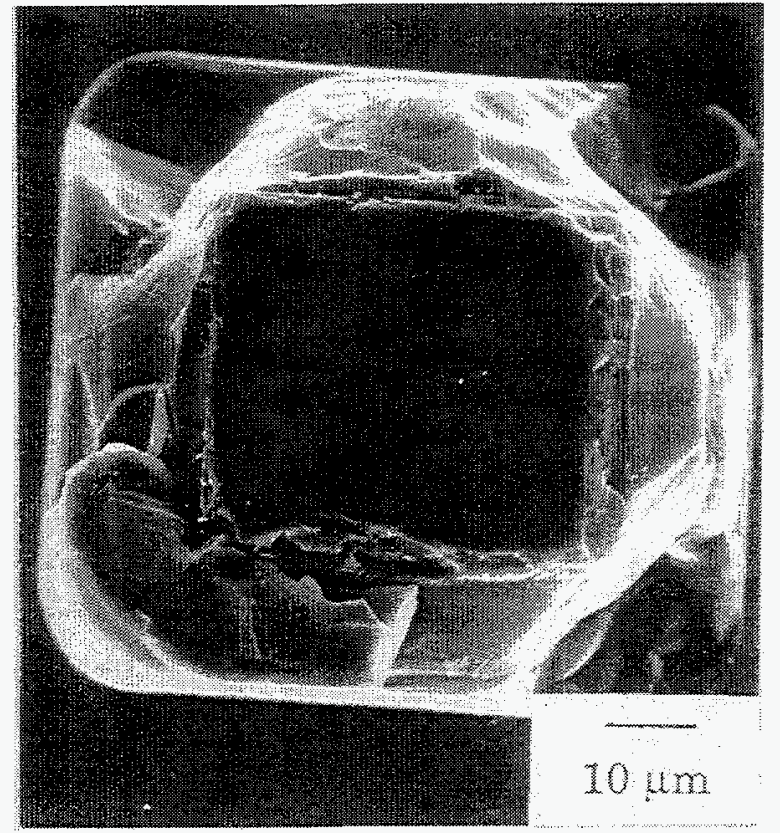

(a)

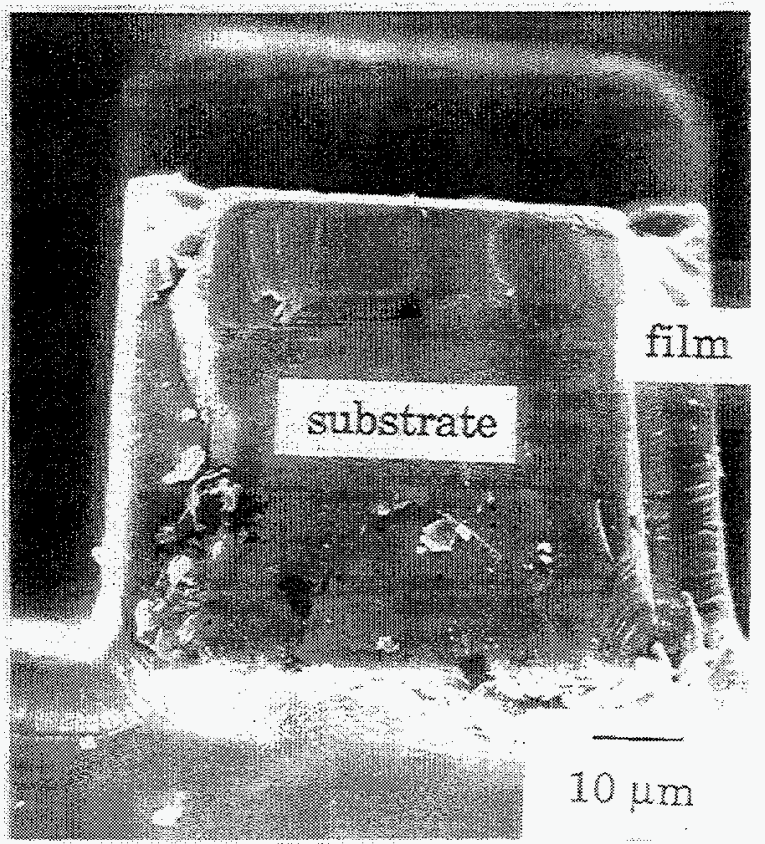

(b)

Figure 3.19. Scanning electron micrographs of mesa-patterned samples where some films peeled off. (a) Top view of the delaminated mesa, (b) Fractured cross section of the partially peeled sample. Films on other mesas are seen in the background. 
For the usual case of a thin film with lateral dimensions much greater than the thickness of the film, the shear and normal stresses on the film/substrate interface are significant only very near the edges of the film as seen in the Fig. 3.17. Except near the edges, the stress state in the film is purely biaxial. However, as the lateral dimension is reduced the stresses near the edge of the film become important. For example, debonding of the film from the substrate is expected to start at the edge. The shear and normal stresses acting on the interface there can cause sliding and decohesion to occur. This would allow the biaxial stress in the film to be relaxed and could cause the film to peel off the substrate. The distribution of shear and normal stresses on the film/substrate interface are also important in cases where the lateral dimensions of the film become comparable with the film thickness. In these cases, the interfacial shear stresses are smaller and they are restricted to act over smaller distances from the edge of the film. As a consequence, the film is not fully loaded and the biaxial stress in the center of the film is less than it would be for a comparable film with large lateral dimensions. This relaxation of the stress in the film is important for patterned thin-film structure.[71]

Delamination is further aggravated by sharp edges in three dimensions. If the stress distribution is non-uniform, i.e., highest at the substrate surface and decreasing towards the free surface of the film, the net moment at the edges will increase, causing the film to delaminate by shear. This effect is more pronounced in films in tension.

A-Si:H films of up to $20 \mu \mathrm{m}$ in thickness were deposited successfully, but thicker films partially peeled off. The SEM photographs of a-Si:H films grown on such structures are shown in Fig. 3.19. Cracks developed around the edges of the mesas due to the high stress concentration on sharp corners, and the films on the top of the mesas buckled. However, the films inside the trench were more stable than those at the top. On plain substrates, films of up to $20 \mu \mathrm{m}$ in thickness remained stable with the residual stress of $-350 \mathrm{MPa}$. It is observed in Fig. 3.19 that films of about half the thickness of the top ones are deposited quite uniformly on the sidewalls and at the bottom of the trenches. Although 
the top part peeled off, the films inside the grooves remained attached firmly. In summary, the selective area deposition helps reduce the substrate bending, but cannot prevent the peel-off. Also, it is suggested that the use of the films inside the trenches might be safer than of the top portion.

\subsubsection{Inverted Pyramid Structure}

As discussed in the previous section, the films inside the trenches appear to be mechanically more stable. Therefore, it would be beneficial to confine films in the trenches or wells rather than to have them on the pedestal. However, it is very difficult to deposit films only at the bottom of the trenches or on the vertical wall and to make reliable electrical contacts on them. An alternative way is to grow films on the slopes of V-shaped holes as shown in Fig. 3.20. In the pyramid structure, the horizontal component of the stress, which is related to the bending moment, is decreased by $\cos \theta$, where $\theta$ is the angle between the horizontal surface and the side, and thus substrate bending will be reduced. Delamination of the films will be minimized as well since the films are confined inside the inversed pyramids. It is like a piece of filter paper sitting inside a funnel. The paper tends to expand outward and is tightly bound to the funnel.

The pyramid structure may also enhance the signal collection in two ways. First, the effective thickness of the film through which the radiation particles travel is increased by $1 / \cos \theta$, hence full $50 \mu \mathrm{m}$ deposition will be unnecessary. Second, the direction of the charge carrier sweep-out is inclined to the incident particle by an angle $\theta$, and thus the recombination of the electrons and holes along the particle track is reduced. This effect was demonstrated with plain geometry a-Si:H diodes.[72] When the detector was tilted at $60^{\circ}$ to the incident radiation, the collected signal increased by a factor of 2 for a high density of charges produced by $\alpha$-particles which deposit $\sim 5 \mathrm{MeV}$ of energy in $\sim 20 \mu \mathrm{m}$ distance. 


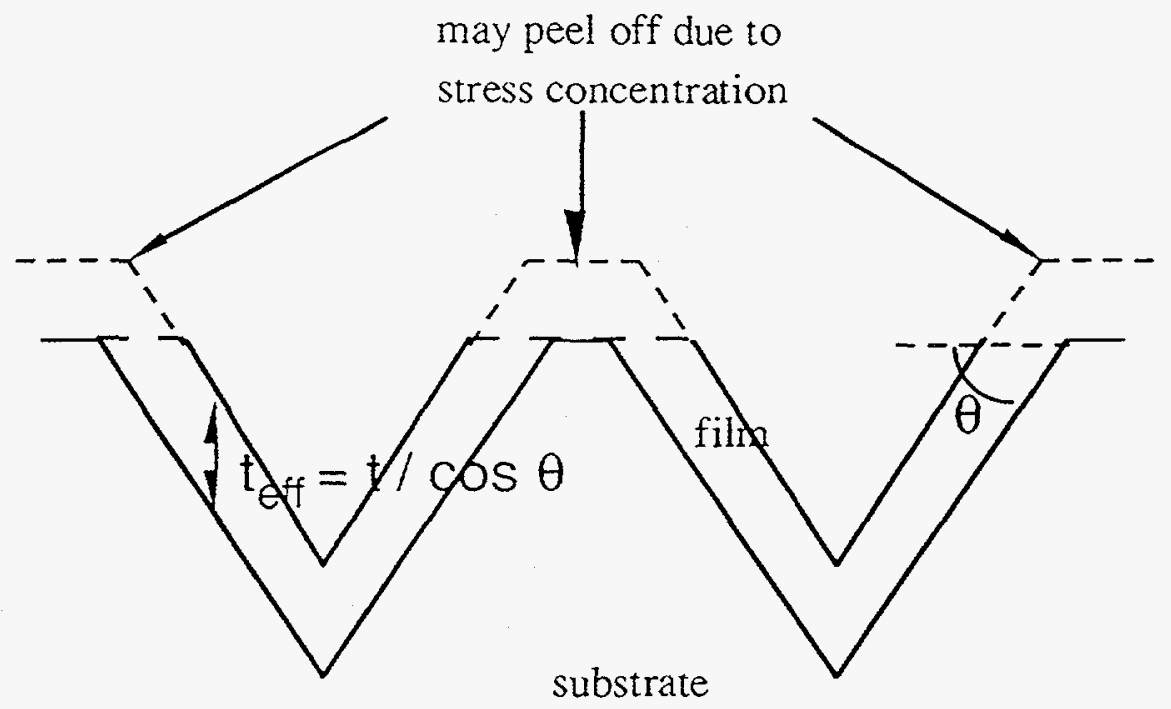

Figure 3.20. Schematic diagram of the cross section of the inverted pyramid pattern

The inverted pyramid structure can be realized by anisotropic etching of the single crystal silicon wafers. First, $1 \mu \mathrm{m}$-thick oxide was grown on a $<100>$ orientation wafer in $1100^{\circ} \mathrm{C}$ pyrogenic steam furnace as a masking layer. $180 \mu \mathrm{m} \times 180 \mu \mathrm{m}$ squares with 20 $\mu \mathrm{m}$ spacing were patterned by photolithography, aligning the major flat of the wafer with a side of the square. The backside of the wafer was coated with photoresist to protect the oxide layer, and the patterned-side oxide layer was etched with 5:1 buffered HF. The wafer was then etched in EDP solution ( $500 \mathrm{ml}$ of ethylenediamine, $160 \mathrm{~g}$ of pyrocatecol, 3 $\mathrm{g}$ of pyrazine dissolved in $160 \mathrm{ml}$ of $\mathrm{H}_{2} \mathrm{O}$ ) until all (111) surfaces were exposed. Etch rate of $(100)$ plane is around $1 \mu \mathrm{m} / \mathrm{min}$. at $105^{\circ} \mathrm{C}$ and the etch rate ratio of $\langle 100\rangle:\langle 110\rangle$ : $<111>$ orientation is $50: 30: 3.173$ ] All the remaining oxides were stripped and the patterned surface was coated with $\mathrm{Cr}$. A-Si:H films of $15 \mu \mathrm{m}$ and $32 \mu \mathrm{m}$ in thickness were deposited and the stress and the fracture cross section were observed. 


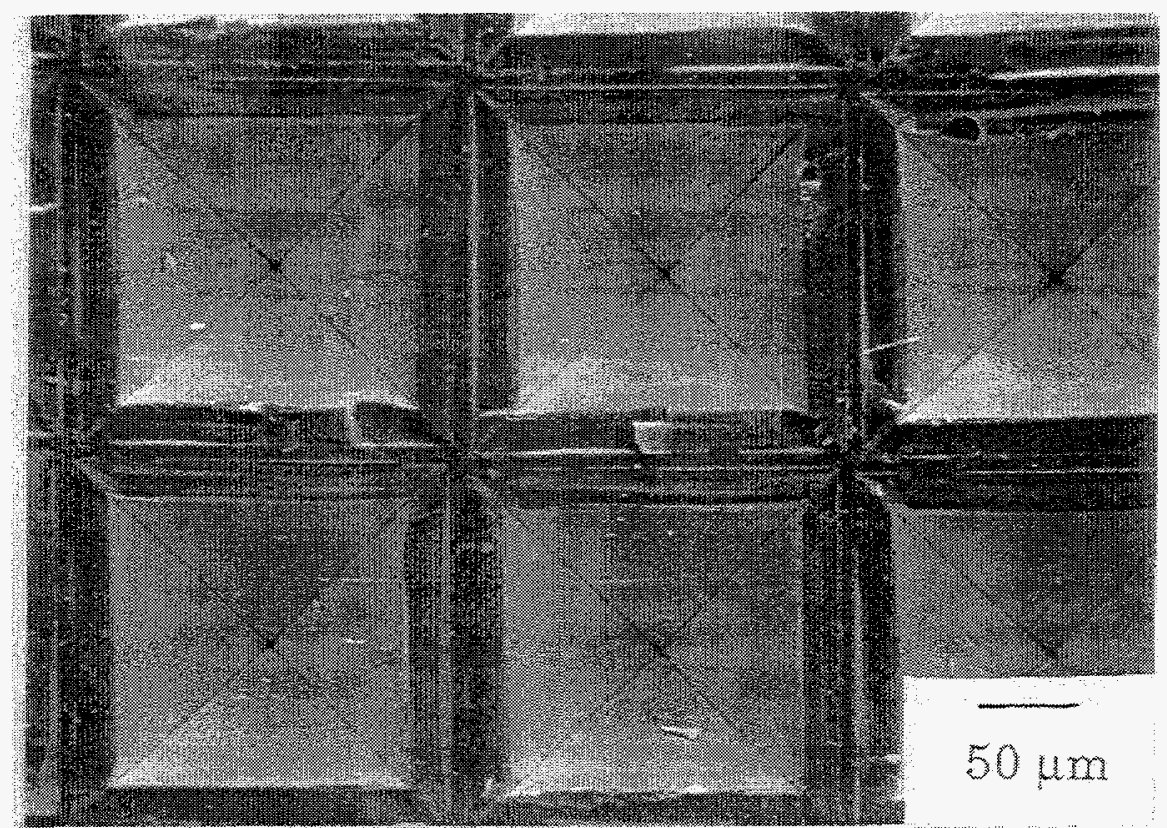

(a)

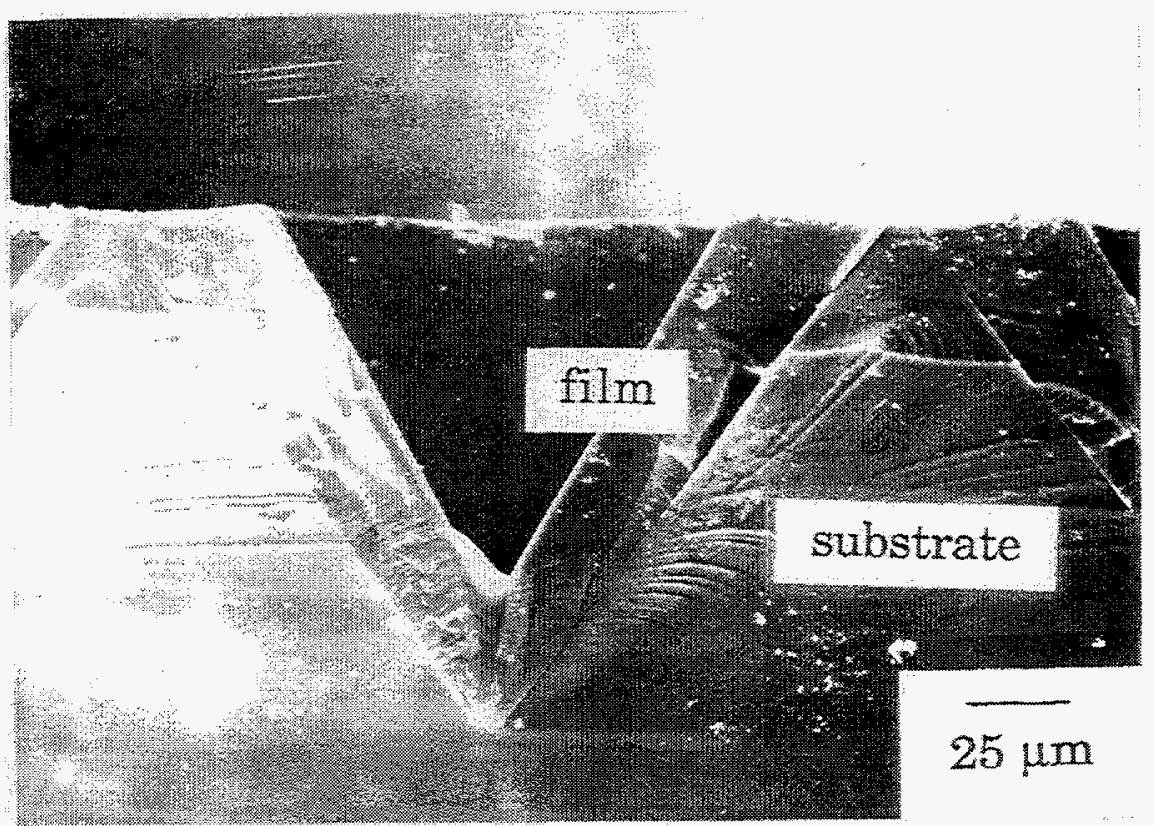

(b)

Figure 3.21. Scanning electron micrographs of inverted pyramid patterns: (a) Top view of $20 \mu \mathrm{m}$-thick a-Si:H films deposited inside the $\mathrm{V}$-shaped holes, (b) Fractured cross section of $32 \mu \mathrm{m}$-thick a-Si:H film deposited on the pyramid-patterned substrate. 


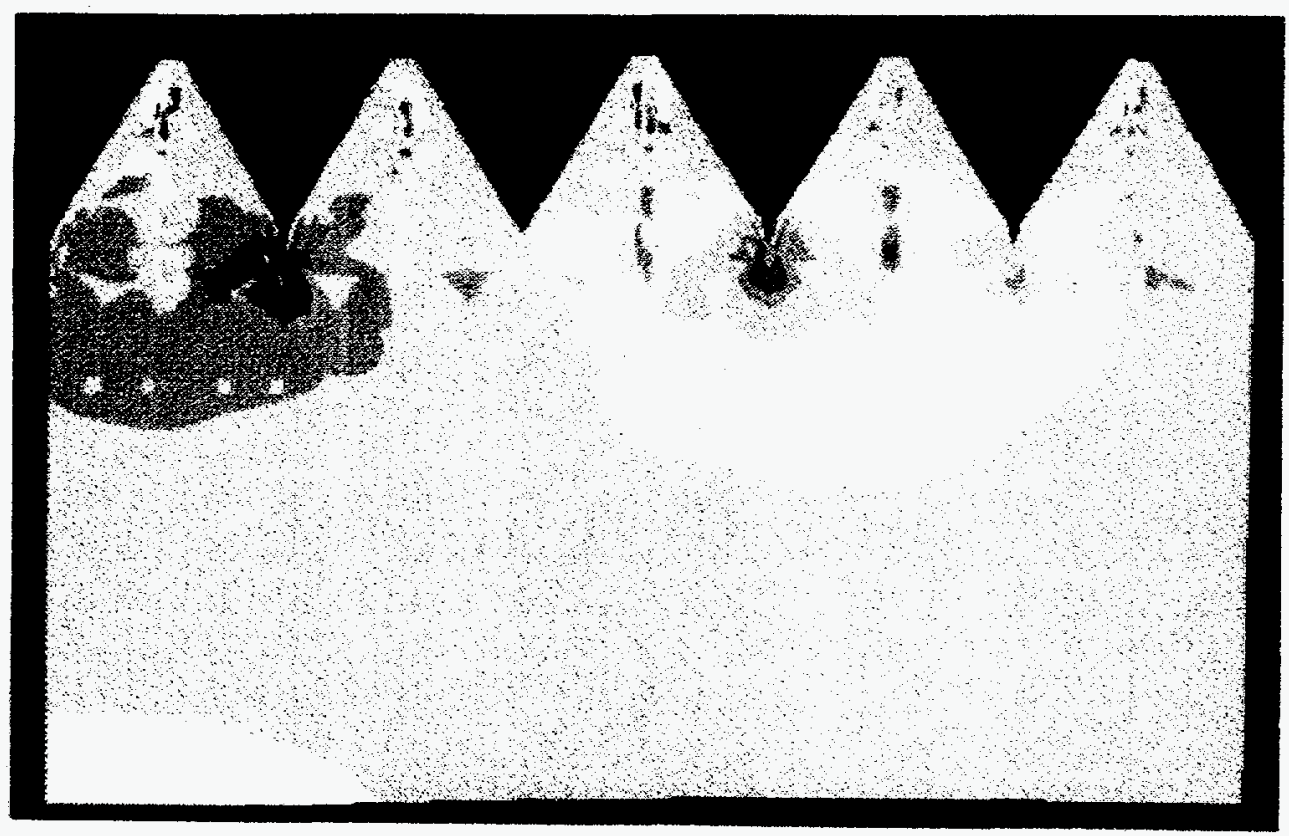

$-0.303 \mathrm{E}+12$

$-0.275 \mathrm{E}+12$

$-0.246 \mathrm{E}+12$

$-0.210 \mathrm{E}+12$

$-0.189 \mathrm{E}+12$

$-0.161 E+12$

$-0.133 \mathrm{E}+12$

(a)

$-0.104 E+12$

$-0.757 E+11$

$-0.479 \mathrm{E}+11$

$-0.189 E+11$

$0.947 \mathrm{E}+10$

$0.379 \mathrm{E}+11$

$0.663 \mathrm{E}+11$

$0.947 \mathrm{E}+11$

$0.123 E+12$

$0.151 E+12$

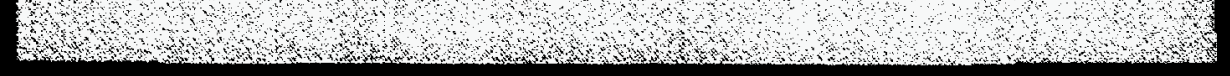

(b)

Figure 3.22. Simulation results of the stress distribution in the pyramidpatterned substrate. The yellow-green color represents zero stress and color change toward red indicates increasing tensile stress. The unit of the stress is dynes $/ \mathrm{cm}^{2}$. (a) When all films remained attached on the substrate, (b) When the top portion peeled off. 
Figure 3.21 shows the SEM pictures of inverted pyramid pattern engraved in a silicon wafer by anisotropic and a-Si:H film deposited inside the pyramids. As seen in the he films on the dead area between the pyramids peeled off because of the large stress concentration as discussed in the previous section. However, this effect is beneficial because it further releases the stress and isolates pixels from each other. The measured apparent stress value was around $100 \mathrm{MPa}$ which is smaller than that of the normal substrate by a factor of $3 \sim 4$.

A computer simulation was performed using the identical geometry and material parameters as those of an actual sample. Figure 3.22 shows a cross sectional view of the stress distribution with a color scale. Fig. 3.22(a) represents a sample with the a-Si:H film deposited evenly on the pyramid patterned substrate, and Fig. 3.22(b) shows a sample with the top portion of the film peeled off due to the stress concentration the sharp peaks. The left side of the substrate was assumed to be fixed to the wall and all the other parts were allowed to deform freely. As illustrated by colors in the figure, most of the stress is taken up by the pyramid part and only a small stress appears in the back of the substrate. If carefully observed, slightly more deformation is noticed in the substrate with all films remained intact (Fig. 3.22(a)) than in the substrate with the top portion peeled off (Fig. $3.22(b))$.

According to these results, the inverted pyramid structure is preferred to the square island pattern for films under high compression. However, there are also some drawbacks in this structure. First, a large stress concentration occurs at the sharp point in the valley of the pyramid as well as near the top and weakens the substrate. This large tensile stress concentrated near the bottom of the pattern can also be visualized by a red color in Fig. 3.22(a). This effect can be moderated to some extent by decreasing the curvature of the point. If a short isotropic etching follows the anisotropic etching, it will decrease the curvature appreciably without degrading the slope much. It will also reduce the intense electric field concentration around the sharp corner which might cause electrical 
breakdown. Second, cross-section of the film is exposed at the top of the pyramid due to the peel-off, and there might be an arc across the surface when a high bias voltage is applied. Therefore, the exposed cross-section needs to be covered by a proper insulator. One of the best candidates is polyimide because it can be easily spin coated and patterned by UV light. 


\subsection{SUMMARY}

A moderate increase in the growth rate to $3.5 \sim 4 \mu \mathrm{m} / \mathrm{hr}$. and residual stress as low as $\sim 100 \mathrm{MPa}$ have been obtained for films deposited at $150^{\circ} \mathrm{C}$ with He-dilution. Postannealing at $160^{\circ} \mathrm{C}$ for 100 hours led to sufficiently low $N_{d}{ }^{*}\left(\sim 7 \times 10^{14} \mathrm{~cm}^{-3}\right)$ without affecting the stress state. The combination of the low temperature growth, He-dilution and post-annealing of the film lends a way to produce a material that has a low residual stress and electrical properties satisfactory for thick $(-50 \mu \mathrm{m})$ radiation detectors.

The structure of the new film is seen to contain voids and tiny crystalline inclusions and is different from the one observed in conventional a-Si:H. Infrared absorption measurement indicated the existence of microvoids, which relax the internal stress, in the low-temperature materials both for He-diluted or pure silane. It also showed that there are tiny crystallites of the order of a few tens of atoms in the He-diluted layers. The lower defect density in the He-diluted material than in the normal a-Si:H has been attributed to the high level of hydrogen incorporation and heavily clustered microstucture, which are associated with the high deposition rate.

A void-rich material is created here with satisfactory electronic quality, which is in contrast with previous conclusions in the literature. Therefore, the He-diluted films prepared by low-temperature deposition and subsequent annealing are preferred over the standard a-Si:H since they have lower residual stress and faster deposition rate with electrical properties comparable to those of the standard films. The high temperature stability of the hydrogen bonds remains an unresolved phenomenon.

Deposition on patterned substrates was attempted to minimize delamination and substrate bending as an alternative to controlling the deposition parameters. A-Si:H films deposited on square islands showed that the apparent stress lowered by $40 \%$ for the island width of $40 \mu \mathrm{m}$, compared to those on the flat surface. However, when the films are thicker than $20 \mu \mathrm{m}$, some of them peeled off at the top of the mesas. When the inverted 
pyramid pattern of $200 \mu \mathrm{m}$ in width was used instead of square mesas, $32 \mu \mathrm{m}$-thick films could be deposited without delamination. The top portion of the film between the pixels peeled off due to the stress concentration, releasing the stress further and isolating pixels from each other. The apparent stress was reduced by a factor of $3 \sim 4$. Computer simulation showed that the stress was imposed mostly on the patterned region, and the rest of the substrate experienced less stress. Inverted pyramid structure seemed to be better than the square mesa structure in the mechanical stability point of view, although it still needs to be improved. Efforts to make the pyramid patterns on substrates other than crystalline silicon are needed for larger area application and for lower cost.

The use of buried p-type layers to reduce the bias voltage necessary to deplete thick amorphous silicon detectors were demonstrated. Model analysis indicated that electron losses owing to the buried p-layers will be within reasonable limits when appropriate doping concentrations are used. Experiments showed that the depletion voltage was reduced to nearly $1 / 2$ in the diodes having a p-layer in the middle. 


\section{References}

[1] G.F.Knoll, "Radiation Detection and Measurements", 2nd Ed., pp.363-364, John Wiley \& Sons (1979)

[2] G.Cho, "Signal and Noise Analysis of a-Si:H Radiation Detector-Amplifier System, Ph.D. Thesis, U.C.Berkeley, (1992)

[3] E.Segre, "Nuclei and Particles", Benjamin-Commings, (1977)

[4] V.Perez-Mendez, in Amorphous and Microcrystalline Semiconductor Devices, edited by J.Kanicki, pp. 297-330, Artech House, Norwood (1991)

[5] S.Qureshi, V.Perez-Mendez, S.N.Kaplan, I.Fujieda, G.Cho and R.A.Street, Mat. Res. Soc. Symp. Proc., 149649 (1989)

[6] R.A.Street, Phys. Rev. B 274294 (1983)

[7] H.Kakinuma, S.Nishikawa, T.Watanabe and K.Nihei, J. Appl. Phys., 5993110 (1986)

[8] J.P.Harbison, A.J.Williams and D.V.Lang, J. Appl. Phys., 554946 (1984)

[9] K.S.Stevens and N.M.Johnson, J. Appl. Phys., $71 \underline{6} 2628$ (1992)

[10] H.L.Priest, H.L.Caswell and Y.Budo, Vacuum, 12301 (1962)

[11] N.M.Johnson, F.A.Ponce, R.A.Street and R.J.Nemanich, Phys. Rev. B, 354166 (1987)

[12] N.M.Johnson, Chap. 7 in Semiconductors and Semimetals, vol.34, Eds. by J.I.Pankove and N.M.Johnson, Academic Press, New York, (1991)

[13] K.Tamahashi, M.Wakagi, F.Ishikawa, T.Kaneko, K.Tamura, A.Satoh and M.Hanazono, Mat. Res. Soc. Symp. Proc., 192621 (1990)

[14] A.Matsuda, T.Kaga, H.Tanaka and K.Tanaka, Jap. J. Appl. Phys., 23 L567 (1984)

[15] C.C.Tsai, J.C.Knights, G.Chang and B.Wacker, J.Appl.Phys., 592998 (1986)

[16] J.C.Knights, R.A.Lujan, M.P.Rosenblum, R.A.Street, D.K. Biegelsen and J.A.Reimer, Appl. Phys. Lett., $38 \underline{5} 331$ (1981) 
[17] U.Kroll, F.Finger, J.Dutta, H.Keppner, A.Shah, A.Howling, J.-L.Dorier and Ch.Hollenstein, Mat. Res. Soc. Symp. Proc., 258135 (1992)

[18] F.J.Kampas, J.Appl.Phys., 536408 (1982)

[19] A.Gallagher and J.Scott, J.Solar Cells, 21147 (1987)]

[20] W.E.Spear and M.Heintze, Phil. Mag. B 54343 (1986)

[21] Y.Ziegler, H.Curtins, J.Baumann and A.Shah, Mat. Res. Soc. Symp. Proc., 149 81 (1989)

[22] Y.Hishikawa, J.Appl.Phys., 623150 (1987),

[23] J.C.Knights and G.Lukovsky, CRC Critical Reviews in Solid State and Materials Sciences, 21211 (1980)

[24] D.A.Doughty and A.Gallagher, Phys. Rev. A, 42106166 (1990)

[25] R.C.Ross and J.L.Vossen, Appl. Phys. Lett., 45239 (1984)

[26] R.A.Street, "Hydrogenated Amorphous Silicon", Cambridge Univ. Press, (1991)

[27] V.Perez-Mendez, G.Cho, I.Fujieda, S.N.Kaplan, S.Qureshi and R.A.Street, Mat. Res. Soc. Symp. Proc., 149621 (1989)

[28] B.Equer and A.Karar, Nucl. Instr. Meth., A271 574 (1988)

[29] J.S.Drewery, G.Cho, W.S.Hong, T.Jing, S.N.Kaplan, A.Mireshghi, V.PerezMendez and D.Wildermuth, Presented in the Spring Meeting of Mat. Res. Soc., April 27 - May 1, 1992

[30] R.A.Street, J.Zesch, M.J.Thompson, Appl. Phys. Lett., 43672 (1983)

[31] M.Stutzmann, D.K.Biegelsen and R.A.Street, Phys. Rev. B, 355666 (1987)

[32] R.A.Street, Appl. Phys. Lett., 411060 (1982)

[33] A.Matsuda, T.Kaga, H.Tanaka and K.Tanaka, Jap. Appl. Phys., 23 L567 (1984)

[34] H.Curtins, N.Wyrsch and A.V.Shah, Electronics Lett., 23228 (1987)

[35] M.Heintze, R.Zedlitz and G.H.Bauer, Mat. Res. Soc.Symp. Proc., 29749 (1993)

[36] U.Kroll, Y.Ziegler, J.Meier, H.Keppner and A.Shah, Mat. Res. Soc. Symp. Proc., 336115 (1994) 
[37] U.Kroll, Private communication

[38] T.Pochet, A.Ilie, F.foulon and B.Equer, IEEE Trans. Nucl. Sci., NS-41 41014 (1994)

[39] R.Meaudre, M.Meaudre, S.Vignoli, P.Roca i Cabarrocas, Y.Bouizem and M.L.Theye, Phil. Mag. B, $67 \underline{4} 497$ (1993)

[40] D.Han and H.Fritzsche, J. Non-Cryst. Sol., 59\&60 397 (1983)

[41] W.B.Jackson and J.Kakalios, Phys. Rev. B, 371020 (1988)

[42] Z.E.Smith and S.Wagner, Phys. Rev. B, 325510 (1985)

[43] Z.E.Smith, S.Aljishi, D.Slobodin, V.Chu, S.Wagner, P.M.Lenahan, R.R.Arya and M.S.Bennett, Phys. Rev. Lett., 572450 (1986)

[44] R.A.Street and K.Winer, Phys. Rev. B, 406236 (1989)

[45] T.J.McMahon and R.Tsu, Appl. Phys. Lett., 51412 (1987)

[46] X.Xu, A.Morimoto, M.Kumeda and T.Shimizu, Appl. Phys. Lett., 52622 (1988)

[47] X.Xu, A.Okumura, A.Morimoto, M.Kumeda and T.Shimizu, Phys. Rev. B, 38 8371 (1988)

[48] T.J.McMahon, Phys. Rev. B, 454512 (1992)

[49] X.Xu, H.Sasaki, A.Morimoto, M.Kumeda and T.Shimizu, Phys. Rev. B, 411049 (1990)

[50] S.Zafar and E.A. Schiff, J. Non-Cryst. Sol., 114618 (1989)

[51] Y.Kitsuno, G.Cho, J.Drewery, W.S.Hong and V.Perez-Mendez, Jap. J. Appl. Phys., 33 Part 1 3A 261 (1994)

[52] B.Bhushan and B.K.Gupta, "Handbook of Tribology", McGraw-Hill Inc. (1991)

[53] A.G.Evans and J.W.Hutchinson, Int. J. Solids Str., $20 \leq 455$ (1984)

[54] B.R.Lawn and T.R.Wilshaw, "Fracture of Brittle Solids", Cambridge Univ. Press, (1974)

[55] S.Timoshenko and J.M.Gere, "Theory of Elastic Stability", 2nd Ed., McGraw Hill, New York (1961) 
[56] G.Lukovsky, J. Non-Cryst. Sol., 76173 (1985)

[57] Y.Hishikawa, K.Watanabe, S.Tsuda, M.Ohnishi and Y.Kuwano, J. Non-Cryst. Sol., 97\&98399 (1987)

[58] M.A.Omar, "Elementary Solid State Physics", pp.114, Addison Wesley, Reading (1975)

[59] R.C.Lord, in Encyclopedia of Physics, Ed. by S.P.Parker, pp.939, McGraw-Hill, New York (1983)

[60] T.M.Devine, in Electrochemical and Optical Techniques for the Study and Monitoring of Metallic Corrosion, Eds. by M.G.S.Ferreira and C.A.Melendres, pp. 389, Kluwer Academic, Holland (1991)

[61] T.M.Devine, ibid., pp. 439

[62] M.H.Brodsky, M.Cardona and J.J.Cuomo, Phys. Rev. B, 163556 (1977)

[63] C.C.Tsai and R.J.Nemanich, J. Non-Cryst. Sol., 35\&36 1203 (1980)

[64] G.Lukovsky, R.J.Nemanich, and J.C.Knights, Phys. Rev. B, 192064 (1979)

[65] P.J.Zanzuchi, Semiconductors and Semimetals, Vol.21 Part B, Ed. by J.Pankove, pp.113, Academic Press (1984)

[66] CRC Handbook of Chemistry and Physics, 48th Ed., Ed. by R.C.Weast, pp.E3, The Chemical Rubber Co., (1967)

[67] W.Beyer, H.Wagner and H.Mell, Solid State Commun., 39375 (1981)

[68] M.Yamaguchi, M.Tachikawa, M.Sugo, S.Kondo and Y.Itoh, Appl. Phys. Lett., 56 $127(1990)$

[69] B.Yacobi, S.Zemon, P.Norris and C.Jagannath P.Sheldon, Appl. Phys. Lett., 51 $\underline{26} 2236(1987)$

[70] E.Suhir, J. Appl. Mech., 53657 (1986)

[71] M.F.Doerner and W.D.Nix, "Stresses and Deformation Processes in Thin Films on Substrate", in CRC Critical Reviews in Solid State and Materials Sciences, $14 \underline{3}$ pp.225 (1988) 
[72] S.Qureshi, "Hydrogenated Amorphous Silicon Radiation Detectors: Material Parameters; Radiation Hardness; Charge Collection", Ph.D. Thesis, U.C. Berkeley, (1991)

[73] R.M.Finne and D.L.Klein, J. Electrochem. Soc., $144 \underline{9} 965$ (1967) 


\section{CHAPTER 4. MICROSTRIP / MICROGAP GAS CHAMBERS}

\subsection{INTRODUCTION}

\subsubsection{Historical Background}

The microstrip gas chamber (MSGC) is a miniaturized version of the positionsensitive proportional counter made with microelectronics technology. The proportional counter was introduced in late 1930s and relies on the phenomenon of gas multiplication to amplify the charge represented by original ion pairs created in the gas.[1] Applications of proportional counters include the detection and spectroscopy of low-energy X-ray and charged particles.

A proportional counter consists of a thin anode wire and a cylindrical cathode surrounding the anode filled with gas. Large values of the electric field occur in the vicinity of the anode wire because of the cylindrical geometry, and the field at a radius $r$ is given by

$$
E(r)=\frac{V}{r \ln (b / a)}
$$

where $V$ is the voltage applied between anode and cathode, $a$ is the anode wire radius, and $b$ is the cathode inner radius. The electrons and ions created by the incident radiation drift to their respective collecting electrodes. During their migration, these charges experience many collisions with neutral gas molecules. Since the mobility of positive ions is very low, the ions are only drifted to the cathode and achieve very little energy between collisions. On the other hand, free electrons are accelerated by the large applied field in the vicinity of the anode and transfer sufficient kinetic energy to the gas molecule during collision, creating a secondary electron-ion pair. The electron liberated by this secondary ionization process will also be accelerated by the electric field can create subsequent 
ionization. The gas multiplication process therefore takes the form of a cascade. Under proper conditions, the number of secondary ionization events can be kept proportional to the number of primary ion pairs formed, and the observed pulse amplitude indicates the number of ion pairs created within the counter.

For position-sensitivity, more than one wire is provided in a proportional counter. Detectors with very large surface area can be constructed by placing a grid of anode wires between two large flat plates that serve as cathodes. The resulting Multiwire Proportional Chamber (MWPC) was first conceived in 1968.[2] Electrons formed by ionization of the gas drift toward the plane of the anode wires. As they approach, they are accelerated toward the nearest wire into its surrounding high-field region where avalanches are formed. Because the signal then appears only on one anode wire, the event is automatically localized in the dimension perpendicular to the wires. For two-dimensional localization, the second coordinate can be obtained by dividing one cathode plate into narrow strips perpendicular to the wires and sensing the induced charge on the nearest strips.

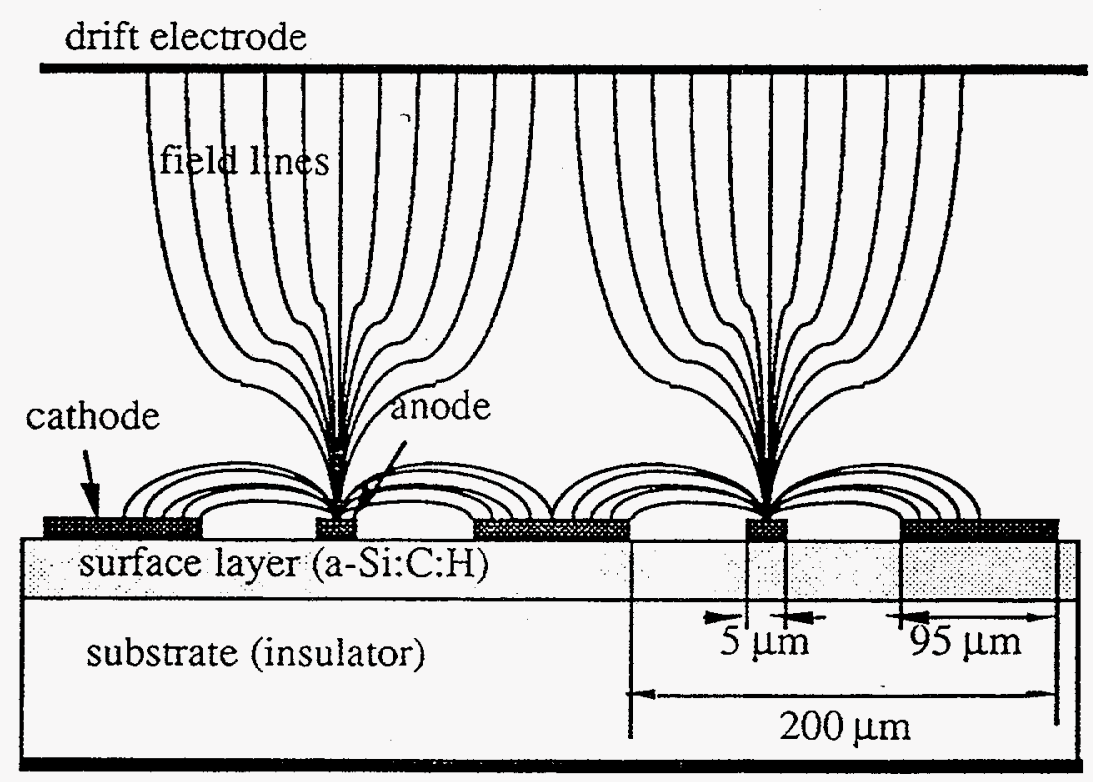

back plane

Figure 4.1. Schematic diagram of the cross section of a gas microstrip detector. 
Nevertheless the MWPC has two major limitations: i) the spatial resolution in the direction orthogonal to the anode wires is limited by the wire spacing,[3] ii) the rate capability is limited by the long positive ion collection time.[4] A smaller wire spacing, however, causes the decrease of the field strength at the wire, and the increased voltages to compensate it are already so high that the operation becomes extremely difficult.

In 1988, Oed proposed microstrip gas chambers fabricated by photolithography techniques to drastically reduce the anode-to-anode pitch and the anode-cathode distance.[5] The wires are replaced by very narrow metallic strips printed on the surface of insulators. Figure 4.1 shows the cross-section of a MSGC. The array of metal strips with alternating potentials shapes the electrical field so that a field strength necessary to produce gas amplification is generated between neighboring strips. An electron produced anywhere in the gas volume will drift along the field lines toward the anode where it will experience an amplification at sufficiently high field strength produced by the intermediate electrodes. The cathode does not contribute to the field intensification due to the relatively long distance from the anode plane, but helps electrons to drift toward the anodes. For this reason, the intermediate electrode is often called the cathode, and the cathode plane in the MWPC structure is called the drift electrode. Owing to its reduced dimension, a MSGC has improved spatial resolution, energy resolution and rate capability.

\subsubsection{Problems in Operating the MSGCs}

In spite of the good spatial resolution, the long term instability and rate-dependent instability of gas gain due to charging on the surface of the insulator impose limitation on the use of the microstrip structure. As mentioned earlier, due to their low mobility relative to that of electrons, the positive ions tend to pile up around the anode wires, increasing the effective width of the anode if the cathode is absent. The increased effective anode width decreases the electric field strength near the anode. Consequently, the gain drops with time 
and eventually the avalanche process is terminated. One solution is to collect and neutralize the positive ions at the cathode. Electrical potential of the cathode is somewhere between those of the anode and the drift electrode, so that the accumulated space charge can be swept out toward that electrode by the potential difference but it does not impede the drift of electrons toward the anodes. However, positive ions landing on the surface of the substrate do not move further if the substrate is insulating. As a consequence, the surface of the insulator will become charged.

The substrate thus requires a certain conductivity to neutralize the positive charges depositing on the surface. At the same time, the surface conductivity should not be too high, because then the leakage current between the anode and cathode is large enough to produce excessive noise above the signal level. It has been shown empirically that the surface resistivity must be within the range of $10^{12} \sim 10^{16} \Omega / \square$ to provide a stable avalanche gain with low noise.[6] Therefore, finding a suitable substrate material with resistivity in the above range has been of primary interest. Among the strategies to achieve the desired resistivity are ion implantation of insulating materials and sputter-coating of thin conducting films.[7-9] Both good long-term stability and high rate capability have been obtained by these methods. However, ion-implantation is much more expensive than ordinary thin-film coating process. Moreover, charging up of electrons at the insulating substrate during implantation makes it difficult to control the uniformity and dosage accurately.[10] Also, electronic conductors are preferred over ion-conducting materials because the ions migrate under a high electric field, resulting in non-uniform resistivity profile in the substrate surface.[11] Sputtering or evaporation of conducting material is relatively easy and cheap, but it is hard to change the resistivity in a broad range since the resistivity is controlled mostly by varying the thickness. 


\subsubsection{Application of $\mathrm{a}-\mathrm{Si}: \mathrm{H}$ and Its Alloys to MSGCs}

Hydrogenated amorphous silicon (a-Si:H) and its alloys are good candidates for a semi-conducting substrate for Microstrip Gas Chambers (MSGCs) due to their good radiation hardness and ability to deposit over a wide area at a low cost. Not only is the bulk resistivity of a-Si:H layer in a suitable range for a $\operatorname{MSGC}\left(\sim 10^{9} \Omega \cdot \mathrm{cm}\right)$, it can also be easily controlled over a wide range by doping or alloying in the gas phase.

However, as reported by Savard et al., intrinsic a-Si:H layers showed variation in surface resistivity and gain with the backplane voltages, $V_{b}$.[12] They attributed such a behavior to the undepleted carriers in a-Si:H, i.e., non-bonding valence electrons. In addition, it has been observed that it takes several hours to reach the equilibrium value of the leakage current between the anode and the cathode.[13] The time-dependent leakage current behavior is characterized by an initial sharp increase upon application of the bias voltage followed by a gradual decay with time. The origin of the time-dependence of the leakage current is not well understood. However, the slow equilibration in an undoped aSi:H sample seems to be due to the release of charge carriers from the dangling bonds under a strong electric field.

Another feature of a-Si:H film is its light sensitivity. The resistivity decreases by 3 or 4 orders of magnitude when exposed to ambient light. Variation of the surface resistivity with ambient light condition will change the leakage current between anodes and cathode, which in turn influences the gas gain. Even when tightly sealed from its surrounding, the photons generated during the avalanche process may affect the gain. Collisions between electrons and neutral gas molecules sometimes produce simple excitation of the gas molecule without creating secondary ionization. These excited molecules do not contribute directly to the avalanche but decay to their ground state by emitting a visible or ultraviolet photon. Under proper circumstances, these photons trigger additional ionization or photoelectric interaction. Such photon-induced events are generally 
undesirable in proportional counters because they can lead to a loss of proportionality and spurious signals.[1]

Therefore, "engineering" of the a-Si:H films was attempted to minimize light sensitivity and the effect of the non-bonding valence electrons (dangling bonds) to improve the performance of the a-Si:H based detectors. One way is to make the electronic conduction in a-Si:H dominated by extrinsic carriers, i.e., dopants. If the number of carriers introduced by doping is much greater than that released from dangling bonds or excited from valence band, the overall leakage current would not be affected by a small fluctuation due to dangling bonds or band-to-band transition. Since the sheet resistance falls below the suitable resistivity range $\left(10^{12} \sim 10^{16} \Omega / \square\right)$ when the number of extrinsic carriers exceeds that of intrinsic carriers (ionizable dangling bonds), it must be compensated by alloying with other elements. One of the best candidates is carbon since it is in the same column in the periodic table as $\mathrm{Si}$ and addition of $\mathrm{C}$ to $\mathrm{Si}$ is known to increase the bandgap and resistivity. In this chapter, test results of gas microstrip detectors having doped a-Si:C:H coatings on a surface are presented and compared with the results obtained from detectors made on insulating glass surfaces and on other semiconducting materials.

\subsection{EXPERIMENTAL Procedures}

An alternating anode and cathode pattern was formed with a $0.2 \mu \mathrm{m}$-thick $\mathrm{Cr}$ layer on a-0.8 mm-thick Corning 7059 glass substrate using the sputtering and lift-off method to ensure a uniform edge profile. The anode and the cathode widths were $5 \mu \mathrm{m}$ and $95 \mu \mathrm{m}$, respectively, and the anode to anode pitch was $200 \mu \mathrm{m}$. The active area of the detector was $15 \mathrm{~mm} \times 20 \mathrm{~mm}$. Layers of a-Si:C:H approximately $100 \mathrm{~nm}$-thick were deposited by the plasma enhanced chemical vapor deposition (PECVD) technique either before or after the 
electrodes were made. The carbon content and doping density in the a-Si:C:H films were controlled by changing the relative flow rates of the $\mathrm{CH}_{4}$ and $\mathrm{B}_{2} \mathrm{H}_{6}$. Ten anode strips were grouped together and biased with a positive voltage. All cathode strips were interconnected and held at a ground potential. The dark surface resistivity was calculated from the sample geometry and the leakage current between anode and cathode. The photoconductivity was measured under 600 lux white light illumination.

The microstrip plate was mounted on a printed circuit board inside an aluminum chamber with an O-ring seal. The drift plane was made of a thin aluminum wire grid and was located $4 \mathrm{~mm}$ above the microstrip plate. $50 / 50 \mathrm{Ar}-\mathrm{CH}_{4}$ or $\mathrm{Ar}-\mathrm{C}_{2} \mathrm{H}_{6}$ mixture was used as the fill gas with a flow rate of $100 \mathrm{sccm}$. The anode readout was capacitively coupled to a charge-sensitive preamplifier. The signal charges were then integrated by a shaping amplifier and processed by a pulse height analyzer. The detectors were tested with a $55 \mathrm{Fe}$ source which emits a $5.9 \mathrm{keV} \mathrm{X}$-ray. Gas avalanche gain, energy resolution, timedependent stability and ageing were studied from the recorded pulse height spectrum.

\subsection{RESUlts AND Discussion}

The dark surface resistivity and the light sensitivity as functions of the $\mathrm{CH}_{4}$ content in the process gas are plotted in Fig. 4.2. The light sensitivity is defined as the ratio of photoconductivity to dark conductivity. The dark resistivity increased and the light sensitivity decreased with the $\mathrm{CH}_{4}$ content. The dark resistivity increased rapidly up to a $\mathrm{CH}_{4}$ content of $30 \%$ and then slowed down.

The increase in the dark resistivity with increasing carbon content is due to the combined effects of reduced carrier mobility and number of carriers participating in the electrical transport. It is reported that the electron drift mobility of a-Si:C:H films having band gap energies of $1.75 \sim 1.9 \mathrm{eV}$ are $1 \sim 3$ orders of magnitude lower than that of pure a- 
Si:H.[14] Therefore, the initial abrupt increase in resistivity with carbon content is believed to be mainly due to the decrease in electron mobility.

The light sensitivity did not change appreciably until the $\mathrm{CH}_{4}$ content is increased to $50 \%$. According to other reports, the optical gap of the material made from $50 \% \mathrm{SiH}_{4}-$ $50 \% \mathrm{CH}_{4}$ mixture is between $1.9 \sim 2.3 \mathrm{eV}$. The maximum value of the optical band gap energy is about $2.7 \mathrm{eV}$ when the atomic fraction of carbon incorporated in the film $X=$ 0.65.[15, 16] This gap energy corresponds to the wavelength of $460 \mathrm{~nm}$ (blue light); thus alloying with carbon solely cannot effectively prevent conductivity changes due to incident photons when illuminated with white light. Moreover, the undoped a-Si:C:H is transparent to UV.

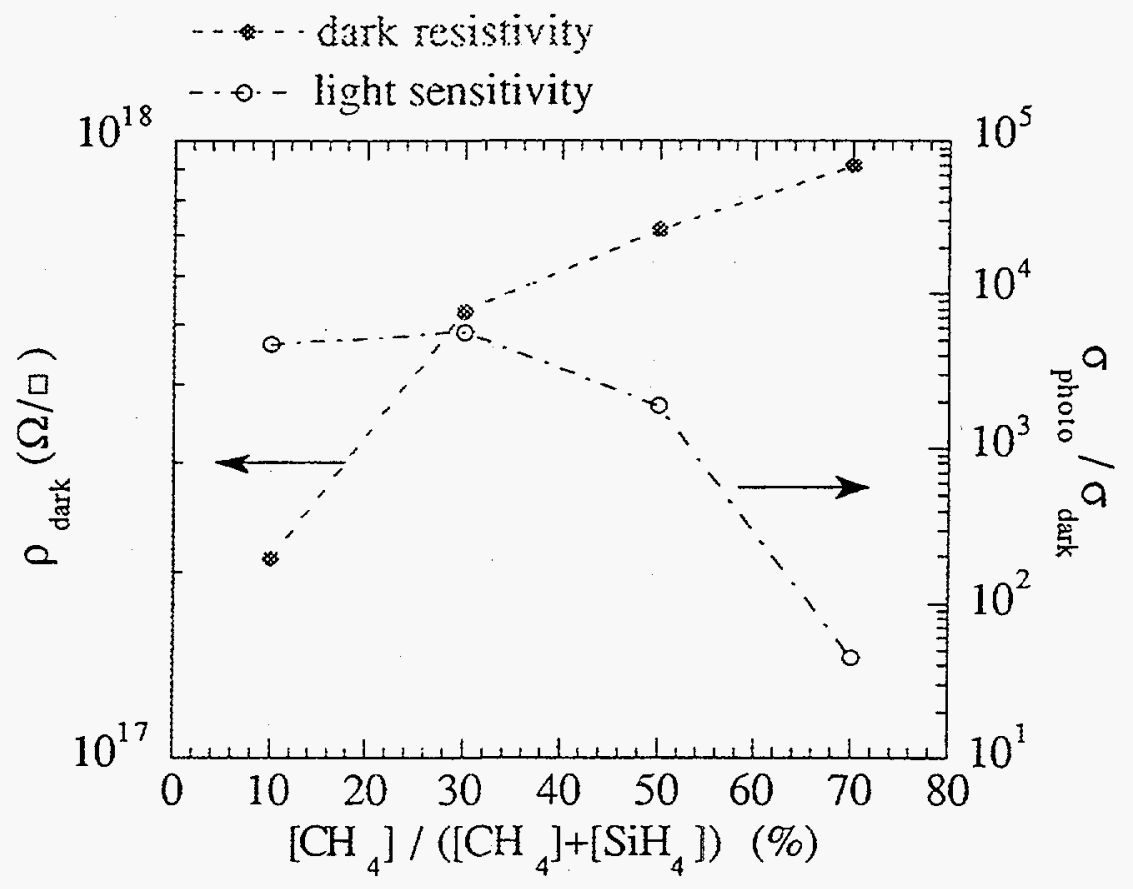

Figure 4.2 Variations of dark surface resistivity and light sensitivity as a function of $\mathrm{CH}_{4}$ content in the source gas. 


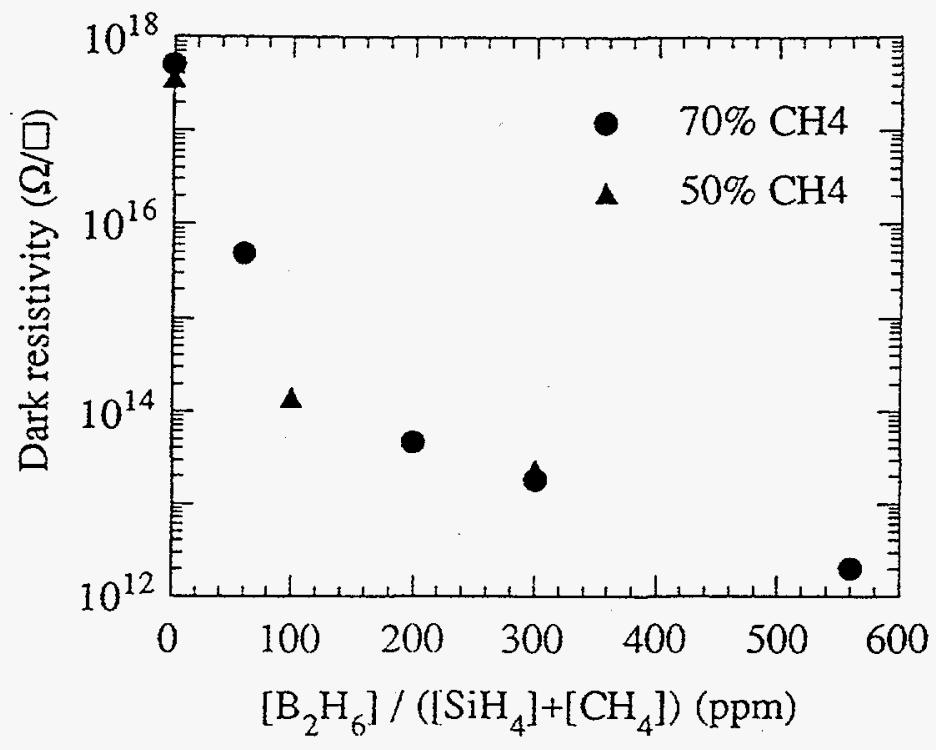

(a)

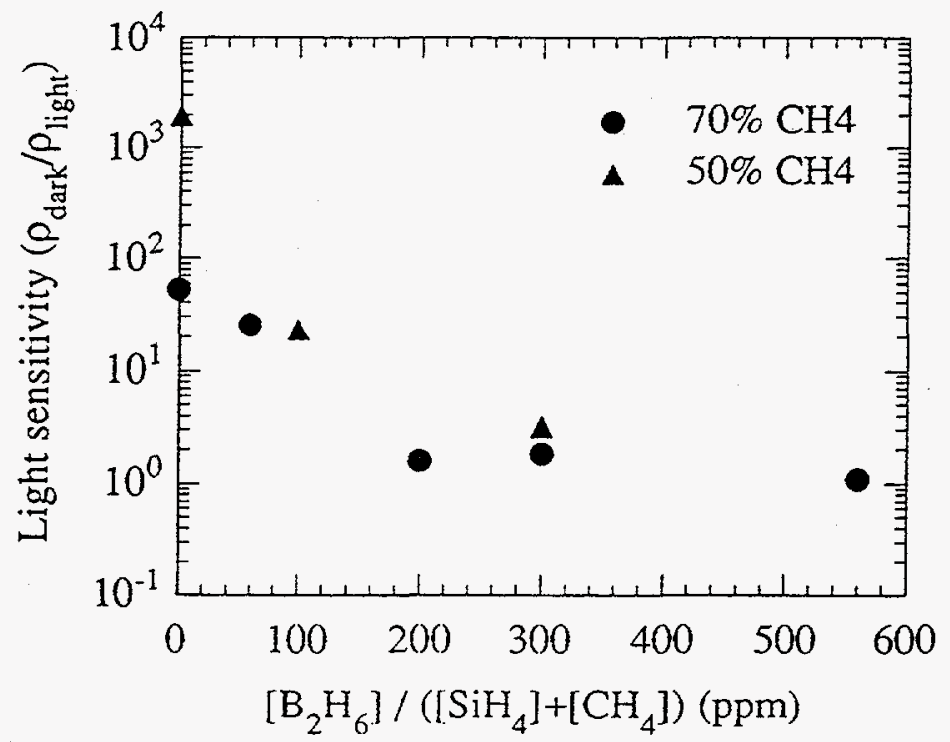

(b)

Figure 4.3. Change in (a) dark resistivity, and (b) light sensitivity with gas phase boron doping density 
In order to suppress the light sensitivity further, gas phase doping was performed. If a semiconductor is heavily doped, the conductivity will be determined by the doping level and not be affected significantly by temperature or illumination conditions. Statistically, if the number of extrinsic carriers introduced by doping exceeds that of the intrinsic carriers, the overall conductivity will not change significantly by a small variation of the number of electron-hole pairs produced by light. Figure 4.3 shows variation in dark resistivity and light sensitivity with the boron doping concentration for samples prepared from gas mixtures containing $50 \%$ and $70 \% \mathrm{CH}_{4}$, respectively. It is clearly seen from both sets of data that the light sensitivity decreases with doping density. With a gas phase doping density of $600 \mathrm{ppm}$, a-Si:C:H deposited from a $30 \% \mathrm{SiH}_{4}-70 \% \mathrm{CH}_{4}$ mixture did not show any variation in resistivity at the given illumination conditions.

Doping also shortens the time to reach equilibrium leakage current between the anode and cathode. Time dependence of the surface resistivity calculated from the leakage current is plotted in Fig. 4.4 for devices with undoped a-Si:H and heavily (600 ppm) pdoped a-Si:C:H layers. The p-doped a-Si:C:H reached its steady-state sheet resistance value within 10 minutes, while it took more than 1 hour in the undoped a-Si:H layer. The origin of the time-dependent leakage current behavior is not well understood. However, the long equilibration time in undoped a-Si:H has to do with the relaxation time of the electrons emitted from the dangling bonds. Street reported the exponential decrease of the leakage current from reverse-biased a-Si:H p-i-n diodes with time.[17]

As discussed in section 2.1.3, the intrinsic a-Si:H is slightly n-type because electrons are released from the dangling bonds under a high electric field. Regarding the dangling bonds as deep traps, the thermal emission rate, $e_{o}$, of carriers is expressed as

$$
e_{o}=\omega_{o} \exp \left[-\left(\frac{E_{c}-E_{t}}{k T}\right)\right]
$$




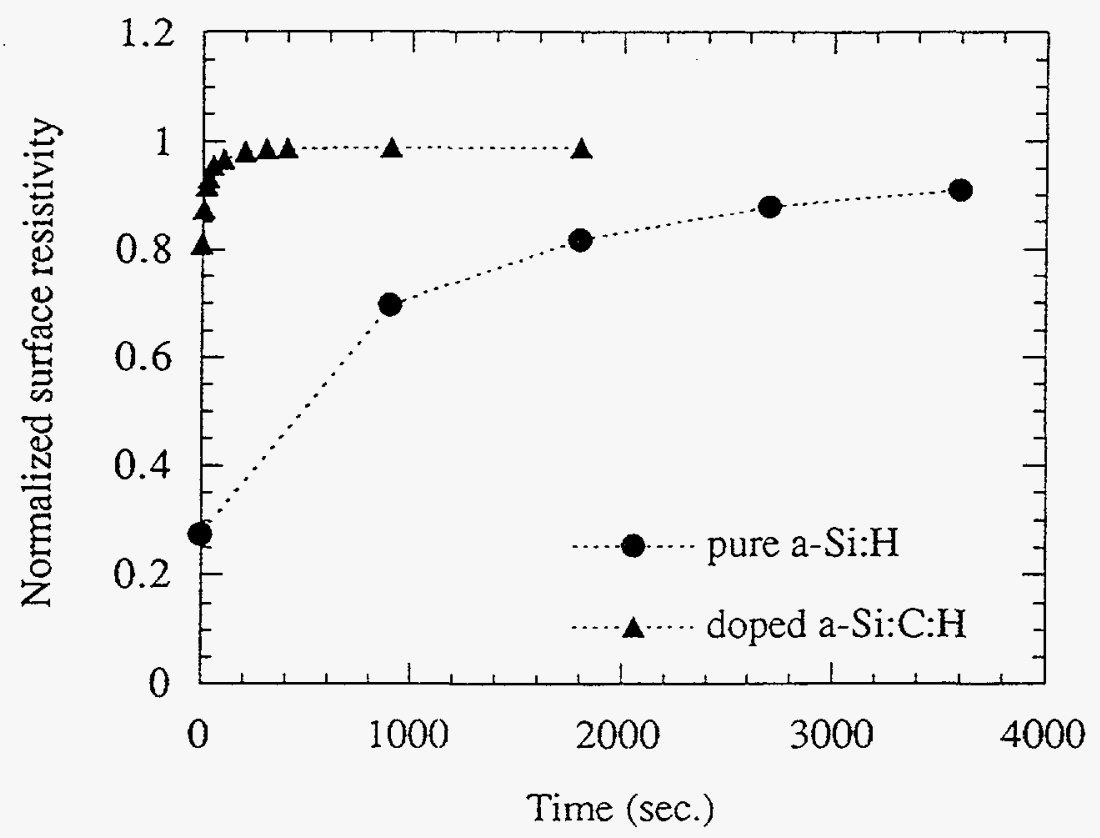

Figure 4.4. Time dependent surface resistivity normalized to a steady-state value.

where $E_{c}$ and $E_{t}$ are the energy levels of the conduction band edge and of the dangling bonds, respectively. Here, $\omega_{o}$ is the attempt-to-escape frequency and is given by

$$
\omega_{o}=k T N\left(E_{c}\right) v_{c} \sigma_{c}
$$

where $v_{c}$ is the free carrier velocity of the mobile electrons and $\sigma_{c}$ is the capture crosssection. $\omega_{o}$ is typically on the order of $10^{12} \sim 10^{13} \mathrm{sec}^{-1}$.

When an electric field is applied, the emission from localized states to the conduction band is enhanced due to potential barrier lowering. The ionization energy is decreased with a square root of the external field. As a result, semiconductors display in high electric fields (over a few thousand volts $/ \mathrm{cm}$ ) an increase of electrical conductivity. 
This phenomenon is called Poole-Frenkel effect.[18] The emission rate for a 1dimensional Coulomb potential well then becomes

$$
e=\omega_{o} \exp \left[-\left(\frac{E_{c}-E_{l}-\Delta E}{k T}\right)\right]=e_{o} \exp \left[\frac{\Delta E}{k T}\right]
$$

where $\Delta E$ is the barrier lowering by the external electric field and is given by

$$
\Delta E=\beta \sqrt{F}
$$

where $F$ is the local electric field and $\beta$ is the Poole-Frenkel constant defined as

$$
\beta=\left(\frac{q^{3}}{\pi \varepsilon_{o} \varepsilon_{\alpha S i}}\right)^{1 / 2}=0.022 \quad \text { in }[\mathrm{eV} \sqrt{\mu \mathrm{m} / \mathrm{V}}]
$$

Therefore, assuming $E_{c}-E_{t} \approx 0.9 \mathrm{eV}$ and an applied field strength of $7 \mathrm{~V} / \mu \mathrm{m}$, which is the typical value for operating the microstrip devices, the emission rate of the carriers from the dangling bonds would be between $3 \times 10^{-4} \sim 3 \times 10^{-3} \mathrm{sec}^{-1}$ at room temperature. The inverse of the emission rate is the relaxation time constant. The time scale of the current decay observed in the a-Si:H microstrip detector is comparable to the relaxation time constant calculated above. It is inferred from these results that the junction between the a-Si:H and the metal electrode is not perfectly ohmic and depletion of charges occurs to some extent.

The above analysis supports the argument that the observed time-dependence of the leakage current is due to the emission of trapped electrons from the dangling bonds under a strong electric field. The dangling bonds occupy energy levels deep down in the band gap ( $\sim 0.9 \mathrm{eV}$ from the conduction band edge), whereas the acceptor impurity level is only $\sim 0.2$ $\mathrm{eV}$ above the valence band edge. The relaxation time of the trapped carriers, which is defined as the time at which the current is 1.1 times its steady-state value, increases exponentially with the position of the energy levels of the deep traps relative to either band 
edge as shown in eqn.(4.2.3). Therefore, it takes more time to reach a steady-state value in the undoped a-Si:H than in the heavily-doped a-Si:C:H where the electrical conduction is dominated by the extrinsic carriers which have a much smaller energy difference.

When tested with a $5.9 \mathrm{keV} \mathrm{X}$-ray from an ${ }^{55} \mathrm{Fe}$ source, a maximum gas gain of $\sim 5000$, rate capabilities of $\sim 10^{5}$ counts $/ \mathrm{mm}^{2} \cdot \mathrm{sec}$, and energy resolution of $20 \%$ FWHM were achieved. As shown in Fig. 4.5, the gas gain increased exponentially with the anode bias until the voltage tripped out. The cathode width was varied between $100 \mu \mathrm{m}$ and 200 $\mu \mathrm{m}$, while the anode width and the spacing berween the anode and the cathode were kept constant. Increasing the cathode width to anode width ratio from 20 to 40 increased the gas gain by a factor of $1.5 \sim 2$ at the expense of the anode-to-anode pitch. Both devices with the a-Si:C:H layer under and over the electrodes worked well, but as reported by others[19, 20], coating the semi-conducting layer over the electrodes greatly simplified the fabrication procedure. In both cases, the gain did not change much either with time or with $V_{b}$. When n-type doping by phosphorus was used instead of boron, the device worked equally well. However, the resistivity of the n-type layer varies abruptly with a small change in the amount of dopant in the gas phase, and the results are hardly reproducible. Thus, p-type doping by boron is preferred over n-type doping by phosphorus.

Changes in normalized cathode current and relative gas gain with backplane voltage, $V_{b}$, are plotted in Fig. 4.6 for four different types of detectors. The sample without surface coating showed a noticeable dependence of the cathode current (avalanche current + leakage current) on $V_{b}$ as reported by others.[21] The pure a-Si:H layer showed a slight dependence on $V_{b}$, but the variation is not as significant as reported by Savard et al.[12] This is probably because the insulating substrate used in this study $(0.8 \mathrm{~mm})$ is 470 times thicker than their $\mathrm{SiO}_{2}$ insulator layer $(1.7 \mu \mathrm{m})$ and the electric field seen by the surface layer is relatively small. The lightly doped sample $\left(60 \mathrm{ppm} \mathrm{B}_{2} \mathrm{H}_{6}\right)$ still had some dependence of the cathode current on $V_{b}$. The heavily doped sample showed no noticeable current variation up to the backplane voltages of $-1200 \mathrm{~V}$, indicating that this layer 
effectively screens the electric field induced by the backplane voltage. As seen from Fig. 4.6(b), the gas gain showed a $V_{b}$-dependence similar to that of the cathode current.

In Fig. 4.7, changes in normalized gas gain are plotted with time for four different types of detectors. For the sample having no coating on the surface, a gain drop of $\sim 30 \%$ was observed within the first 10 minutes, because the surface resistivity $\left(\sim 10^{17} \Omega / \square\right)$ is higher than the optimum range. For the detector with pure a-Si:H coating, the gain decreased first and then increased slightly as time passed. For heavily p-doped a-Si:C:H, the gain remained constant over a period of a week, indicating that the coated layer effectively prevents the positive ions from accumulating on the detector surface.

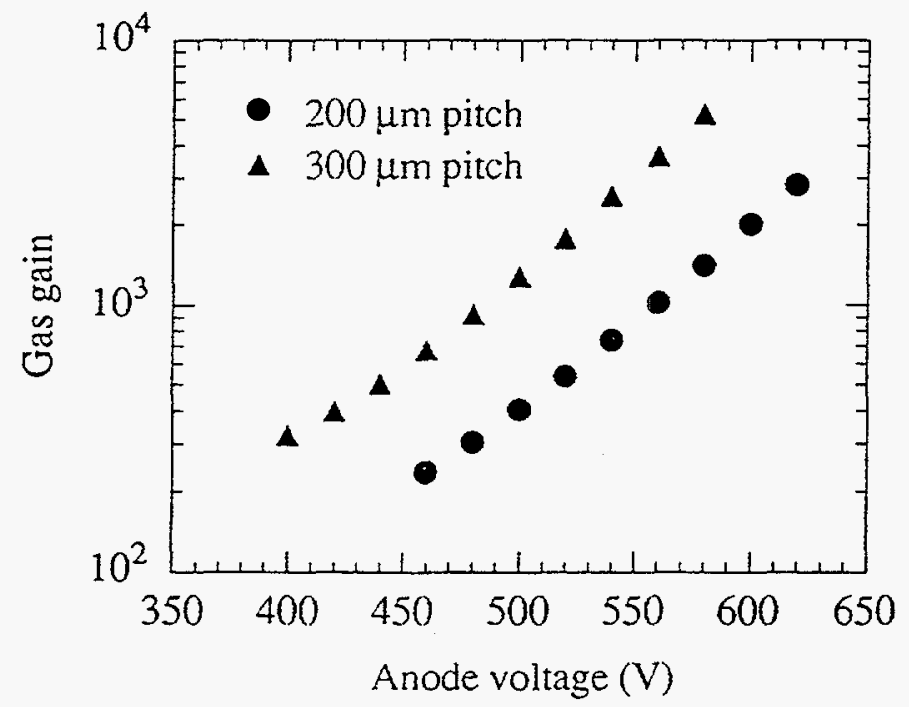

Figure 4.5. Gas gain as a function of the anode voltage for detectors having two different configurations: one with an anode-to-anode pitch of $200 \mu \mathrm{m}$ and cathode-to-anode width ratio of 20 , and the other with a pitch of 300 $\mu \mathrm{m}$ and width ratio of 40 . 


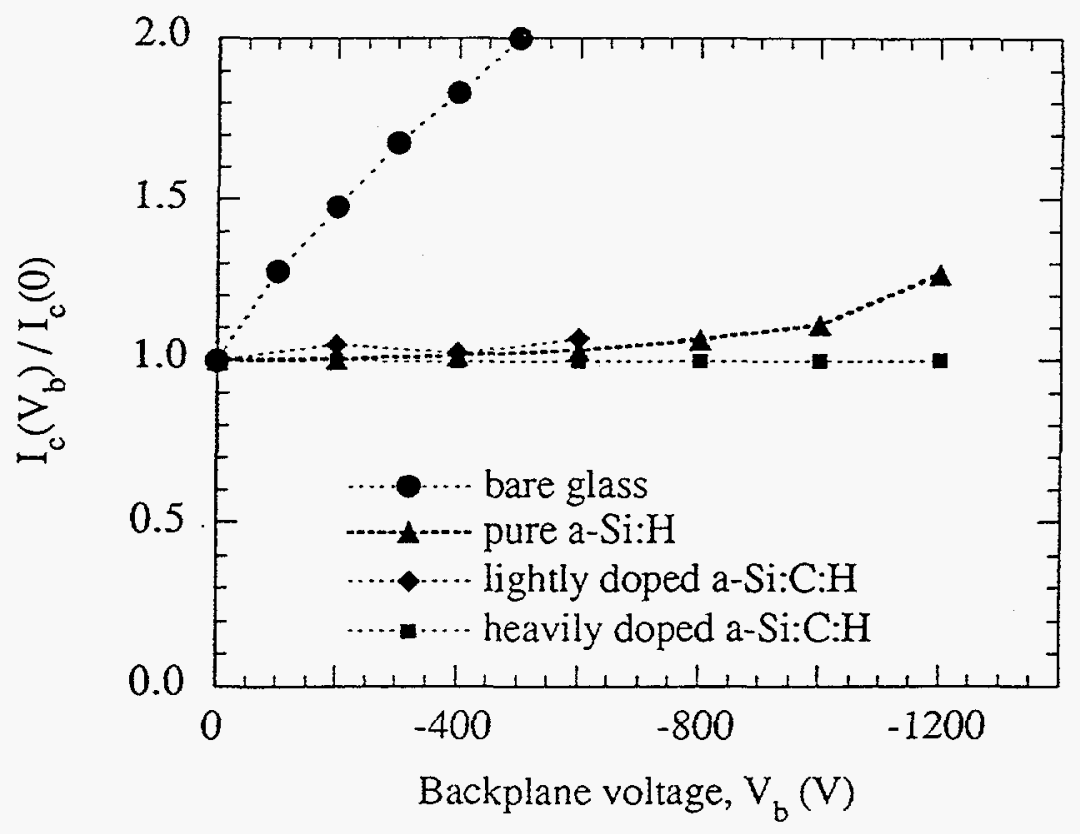

(a)

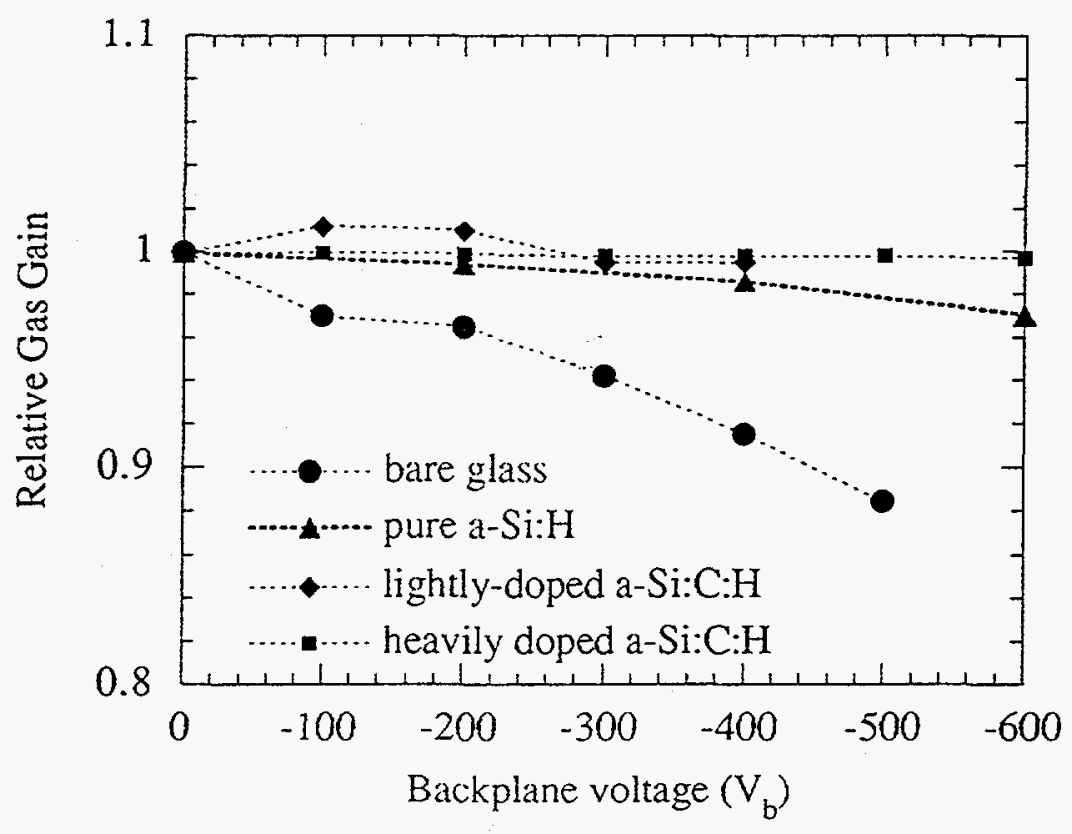

(b)

Figure 4.6. Backplane voltage dependence of (a) normalized cathode current, (b) relative gas gain. 


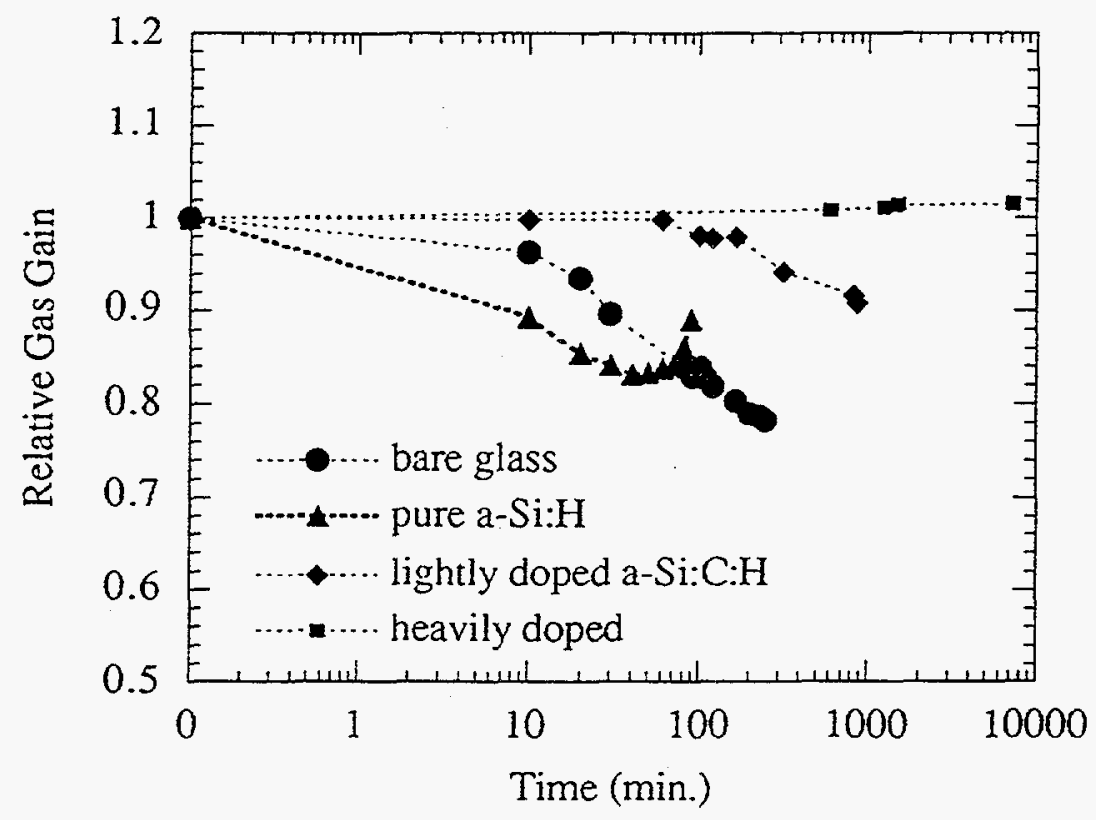

Figure 4.7. Time dependence of the relative gas gain.

For comparison, we also tested MSGCs made on S8900 glass, which has electronic conductivity. The results are shown in Fig. 4.8. Sample configurations and test conditions are summarized in Table IV-1. Performance of the detectors with a-Si:C:H layer was comparable to that with electronic-conductive glass. The sheet resistance was $\sim 10^{12}$ $\Omega /$ in both devices. The samples showed similar gain behavior irrespective of the coating material and fill gas. Only the increase of the cathode-to-anode width ratio from 20 to 40 improved the gas gain by a factor of $1.5 \sim 2$ at the expense of the anode-to-anode pitch. Clearly, a doped a-Si:C:H film can be a good alternative to ion-implantation or semiconducting glass. 


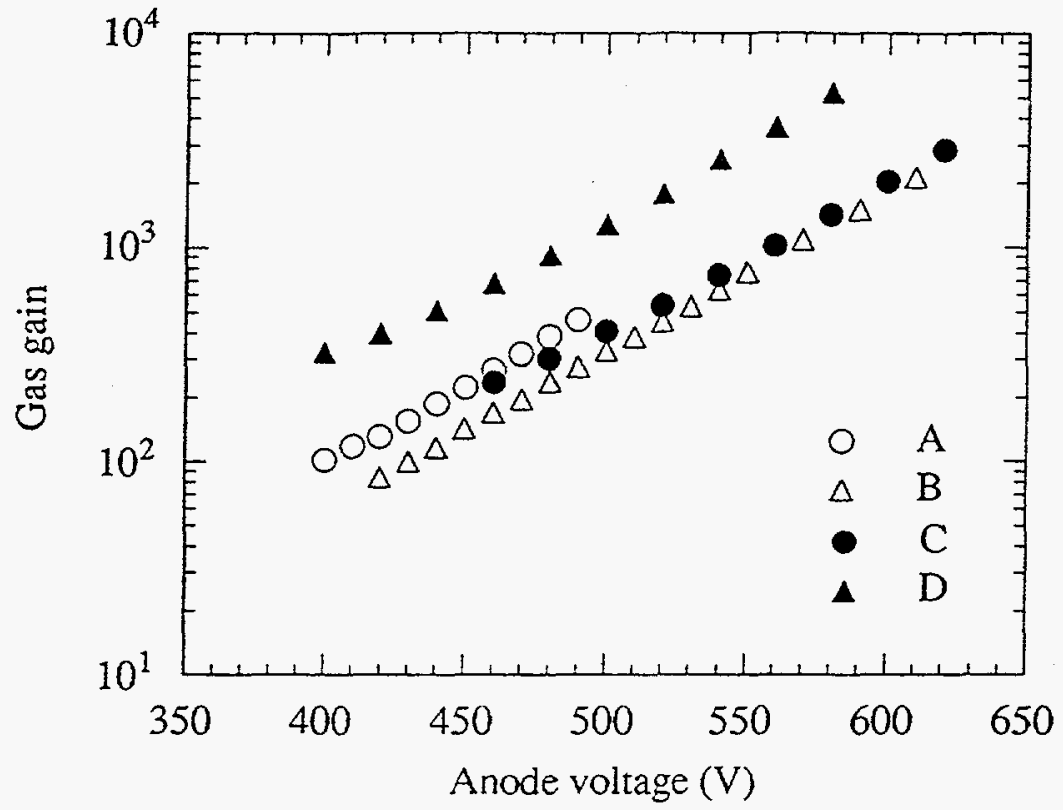

Figure 4.8. Gas gains of MSGCs having two types of surface layers in various gas mixtures. Sample configurations and test conditions are listed in Table IV-1.

Table IV-1. Sample configurations and test conditions of various detectors in Fig. 4.8.

\begin{tabular}{cccccc}
\hline Sample & Surface layer & Pitch & $\begin{array}{c}\text { Cathode-to-anode } \\
\text { width ratio }\end{array}$ & Fill gas & $\begin{array}{c}\text { Drift } \\
\text { voltage }\end{array}$ \\
\hline A & S8900 glass & $200 \mu \mathrm{m}$ & 9 & $\mathrm{Ar}-\mathrm{CH}_{4}$ & $-200 \mathrm{~V}$ \\
B & S8900 glass & $200 \mu \mathrm{m}$ & 9 & $\mathrm{He}-\mathrm{C}_{2} \mathrm{H}_{6}$ & $-600 \mathrm{~V}$ \\
C & p-doped a-Si:C:H & $200 \mu \mathrm{m}$ & 20 & $\mathrm{Ar}-\mathrm{C}_{2} \mathrm{H}_{6}$ & $-1000 \mathrm{~V}$ \\
D & p-doped a-Si:C:H & $300 \mu \mathrm{m}$ & 40 & $\mathrm{Ar}_{2} \mathrm{C}_{2} \mathrm{H}_{6}$ & $-1000 \mathrm{~V}$ \\
\hline
\end{tabular}




\subsection{AGEING}

Ageing is another problem in gaseous devices, which limits high rate capability. Permanent damage to the electrodes after extended irradiation, with a sever degradation of performance, has been associated with the production in the avalanches of polymeric compounds that stick to anodes and cathodes and perturb the counting action or induce discharges.[22] Possibly because the smaller effective area used for charge multiplication on the anode strips or to a more effective polymerization process, MSGCs have been found to be particularly prone to fast ageing. $[23,24]$

Two MSGC devices, with and without p-doped a-Si:C:H coating on the anodecathode strips, were irradiated by an $X$-ray tube at a rate of $1.8 \times 10^{5} \mathrm{~mm}^{-2} \mathrm{sec}^{-1}$. The cathode and backplane voltages of $-460 \mathrm{~V}$ and the drift voltage of $-1460 \mathrm{~V}$ were applied, and the anodes were at ground potential. A $50 / 50$ Ar- $\mathrm{C}_{2} \mathrm{H}_{6}$ mixture was used as fill gas with a flow rate of $76 \mathrm{sccm}$. The X-ray tube was turned off at fixed intervals and the detector was tested using an ${ }^{55} \mathrm{Fe}$ source. The ageing rate, $R$, is defined as

$$
R=-\frac{1}{G_{0}} \frac{d G}{d Q} \quad \text { in }[\% / \mathrm{C} / \mathrm{cm}]
$$

where $G_{o}$ is the initial gas gain, $G$ is the gas gain at a time $t$, and $Q$ is the collected charge per unit length of an anode strip until the time $t$. Figure 4.9 shows the gain variation with collected charge. The ageing rate was calculated to be $1.3 \times 10^{5} \% / \mathrm{C} / \mathrm{cm}$ for the detector without a-Si:C:H coating, while it was only $5 \times 10^{4} \% / \mathrm{C} / \mathrm{cm}$ for the detector overcoated with a-Si:C:H. Since the a-Si:H coats uniformly on the metal strips following the surface contour, it rounds off any sharp corners and edges. This rounding-off is believed to reduce accumulation of polymeric deposits and improves ageing characteristics. 


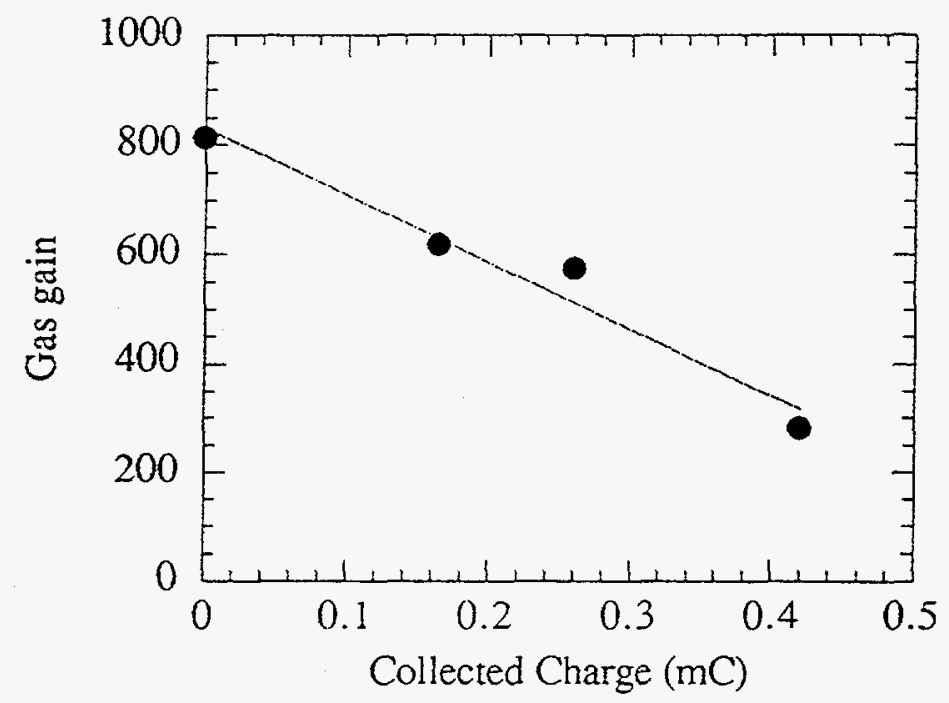

(a)

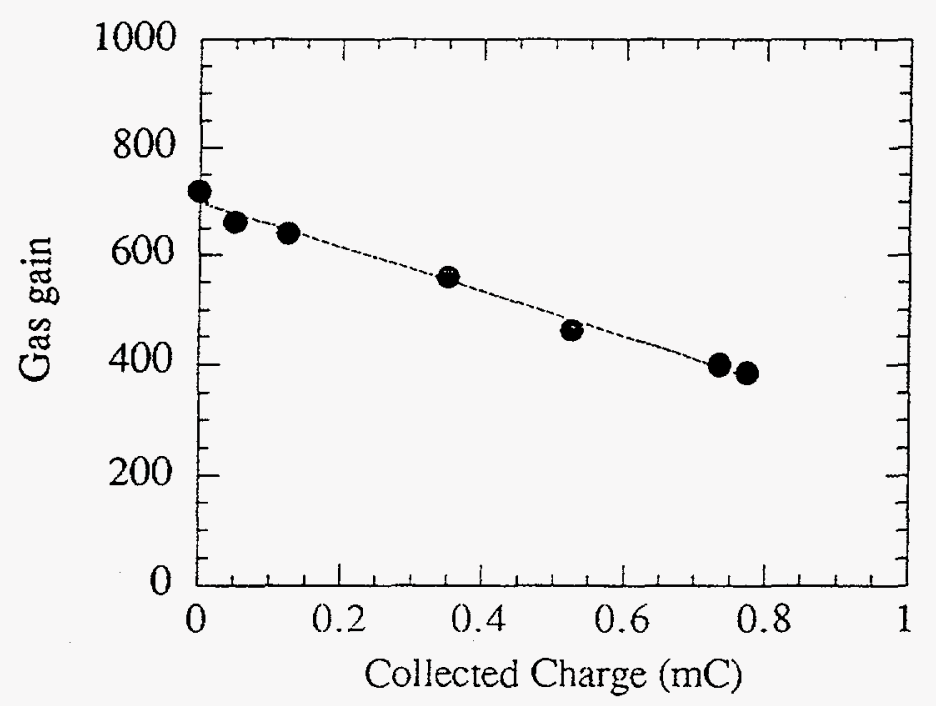

(b)

Figure 4.9. Gain variation as a function of the collected charge per unit length of an anode strip for (a) detector on bare glass (no coating), and (b) detector overcoated with p-doped a-Si:C:H. 


\subsection{Microgap Chamber (MGC)}

A new structural design of a micro-scale gas-filled detector, namely the "microgap chamber (MGC)" was proposed by Angelini et al., in an effort to improve the performance of the MSGC.[25] The cathode strips between two anodes were replaced by continuous sheet placed under the anodes. The anodes were separated from the cathode plate by thin $(\sim 2 \mu \mathrm{m})$ insulator strips only a few microns wider than the anode strips. Figure 4.10 shows the configuration of a microgap chamber. The MGC has shown high rate capability $\left(\sim 10^{7}\right.$ counts $\left./ \mathrm{mm}^{2} \cdot \mathrm{sec}\right)$ and short-term gain stability against surface charging.

Since very high electric fields are imposed between the cathode and anode strips, the thickness and resistivity of the insulating layer must be large enough to suppress the leakage current. $\mathrm{SiO}_{2}$ has been commonly used as this spacer layer. However, the maximum gas gain obtained has been limited by the thickness of the $\mathrm{SiO}_{2}$ layer.[26] The thicker insulating layer gives increased gas gain by allowing a greater distance in which the avalanche may develop, and improves the signal to noise ratio due to the decreased detector capacitance.
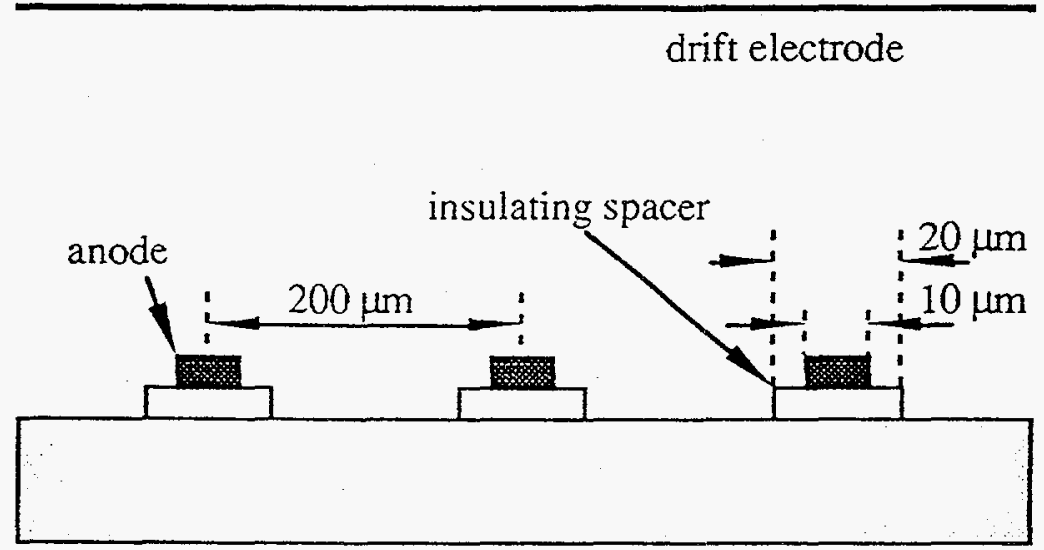

conducting substrate (cathode)

Figure 4.10. Structure of a microgap gas chamber 
Deposition and etching of $\mathrm{SiO}_{2}$ films thicker than $2 \mu \mathrm{m}$ have often been difficult because of a slow deposition rate $(\sim 0.5 \mu \mathrm{m} / \mathrm{hr}$.) and non-uniform etch profile in small structures. Undoped a-Si:C:H prepared from $10 / 90 \mathrm{SiH}_{4}-\mathrm{CH}_{4}$ mixture is an attractive candidate for thick $(\sim 5 \mu \mathrm{m})$ insulating layer since it has a bulk resistivity similar to that of $\mathrm{SiO}_{2}\left(10^{12} \sim 10^{14} \Omega \cdot \mathrm{cm}\right)$, faster growth rate $(2.3 \mu \mathrm{m} / \mathrm{hr}$. $)$ and better etch characteristic in $\mathrm{SF}_{6}-\mathrm{He}$ plasma than those of $\mathrm{SiO}_{2}$.

A $4.6 \mu$ m-thick layer of undoped a-Si:C:H was deposited onto a low resistivity $(\sim 5$ $\mathrm{m} \Omega \cdot \mathrm{cm}$ ) Si wafer using PECVD technique. The low resistivity wafer serves as a cathode. The substrate temperature was $190^{\circ} \mathrm{C}$ during the deposition and all the other deposition parameters were the same as described in section 2.1.4. An aluminum layer of $1 \mu \mathrm{m}$ thickness was then sputter-deposited onto the a-Si:C:H layer. The Al layer was patterned by standard photolithography technique and reactive-ion etching (RIE) to produce $10 \mu \mathrm{m}$ wide anode strips. The underlying a-Si:C:H layer was then patterned to $20 \mu \mathrm{m}$-wide support pedestals for the anode strips. For comparison, an MGC structure is also built on $\mathrm{SiO}_{2}$ surface. The low resistivity $\mathrm{Si}$ substrate was heated in $1100^{\circ} \mathrm{C}$ pyrogenic steam for 6 hours to produce $1.5 \mu \mathrm{m}$-thick surface oxide layer. Al electrodes were metalized and patterned in the same way as in the a-Si:C:H detector. The detectors were tested using a gas mixture of Ar-Ethane (50/50) and an ${ }^{55} \mathrm{Fe} \mathrm{X}$-ray source.

In Fig. 4.11, the measured gas gains as functions of anode bias voltage at a constant drift voltage $(-1000 \mathrm{~V})$ for both the a-Si: $\mathrm{C}: \mathrm{H}$ and $\mathrm{SiO}_{2}$ detectors with the substrate (cathode) ground. The detector with a-Si:C:H as an insulator showed a larger gain at the same anode voltage than the one with $\mathrm{SiO}_{2}$. This is primarily due to the increased thickness of the insulating support. A gas gain of $\sim 2000$ and an energy resolution of $17 \%$ FWHM were achieved with a cathode-anode voltage of $520 \mathrm{~V}$ from the MGC having a$\mathrm{Si}: \mathrm{C}: \mathrm{H}$ insulator. 


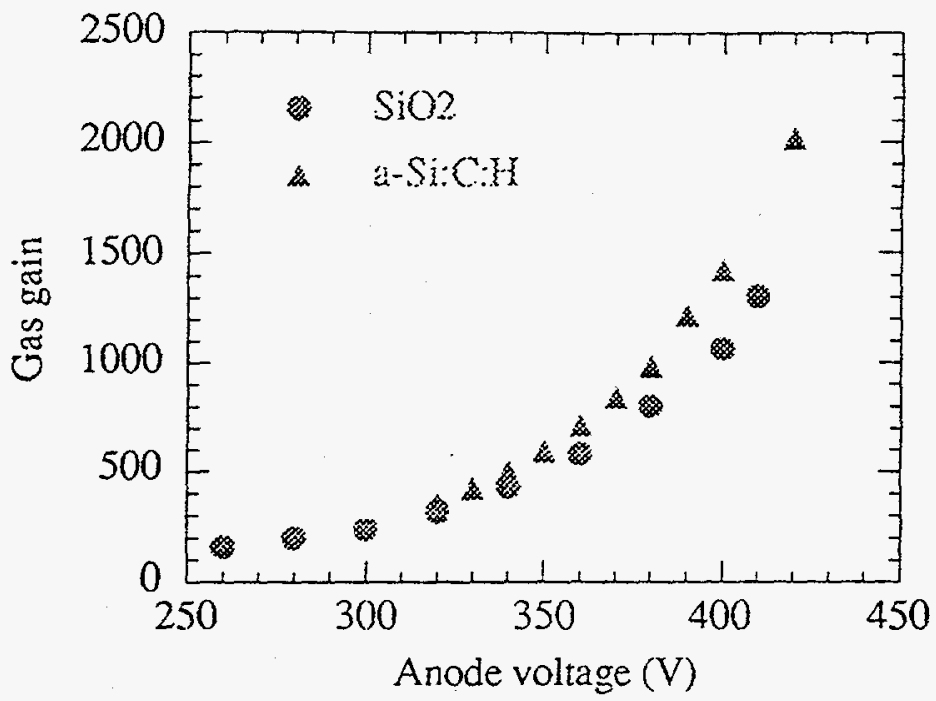

Figure 4.11. Gas gains as functions of the anode voltage for detectors with $1.5 \mu \mathrm{m}$-thick $\mathrm{SiO}_{2}$ and with $4.6 \mu \mathrm{m}$-thick a-Si: $\mathrm{C}: \mathrm{H}$ as insulating layers.

This gain was believed to be limited by small misalignment of the electrodes, seen under a microscope. Since the position accuracy of the contact aligner used to fabricate this detector is only $2 \mu \mathrm{m}$, it is hard to position the $10 \mu \mathrm{m}$ wide anode precisely at the center of the $20 \mu \mathrm{m}$ wide pedestal. Higher gains are expected with improved fabrication techniques. As an altemative to $\mathrm{SiO}_{2}$ as an insulating material, a-Si:C:H is an attractive candidate due to the larger deposition rate possible, lower cost of fabrication, and the similar resistivity compared with $\mathrm{SiO}_{2}$.

\subsection{SUMMARY}

A-Si:H and its carbon alloy coatings were studied for applications to gas microstrip detectors in order to control gain instabilities due to charges on the substrate. Thin 
$(0.1 \sim 0.2 \mu \mathrm{m})$ a-Si:H films have been doped with boron and alloyed with carbon in order to control light sensitivity and time-dependence of its resistivity. The surface resistivity was successfully controlled in the range of $10^{12} \sim 10^{17} \Omega / \square$ and the light sensitivity was minimized by changing the relative amount of the carbon content and doping level in the film. The p-doped a-Si:C:H layers reached a steady-state leakage current value much faster than the undoped a-Si:H. When tested with an ${ }^{55} \mathrm{Fe}$ source, a maximum gas gain of $\sim 5000$, rate capabilities of $10^{5}$ counts $/ \mathrm{mm}^{2} \cdot \mathrm{sec}$, and energy resolution of $\leq 20 \%$ FWHM were achieved. The gain of the detector with a-Si: $\mathrm{C}: \mathrm{H}$ coating $\left(70 \% \mathrm{CH}_{4}\right.$ and $600 \mathrm{ppm}$ $\left.\mathrm{B}_{2} \mathrm{H}_{6}\right)$ remained constant over a week of operation. Performance of the detectors with a$\mathrm{Si}: \mathrm{C}: \mathrm{H}$ layer was comparable to that with electronic conducting glass.

A preliminary ageing result was obtained based upon a prolonged irradiation using an X-ray tube. The detector having p-doped a-Si:C:H layer overcoated on the microstrip structure aged more slowly by approximately a factor of three compared to the one prepared on a bare glass substrate.

High resistivity a-Si:C:H layers prepared from $10 / 90 \mathrm{SiH}_{4}-\mathrm{CH}_{4}$ mixture were applied to microgap gas chambers in an effort to achieve higher gas gains by increasing the thickness of the insulating support pedestal for the anode strips. A $4.6 \mu \mathrm{m}$-thick layer of aSi:C:H was successfully deposited and patterned to $20 \mu \mathrm{m}$-wide pedestals. A gas gain of 200 was achieved, and an energy resolution obtained was $\sim 17 \%$ FWHM. These results showed that a-Si:C:H is good alternative to ion-implanted or semiconducting glass for microstrip detectors, or to $\mathrm{SiO}_{2}$ for microgap chambers, due to the wide range of surface resistivities possible, the ease with which large area may be covered and the low cost of fabrication. 


\section{References}

[1] G.E.Knoll, "Radiation Detection and Measurement", 2nd Ed., pp.160, John Wiley and Sons, New York (1989)

[2] G.Charpak, R.Bouclier, T.Bressani, J.Favier, and C.Zupancic, Nucl. Instr. Meth., $62262(1968)$

[3] F.Angelini, R.Bellazzini, A.Brez, M.M.Massai, G.Spandre and M.R.Torquati, Presented at the 1989 Wire Chamber Conference, Vienna, Feb. 1989

[4] F.Sauli, CERN Yellow Report, 1977

[5] A.Oed, Nucl. Instr. Meth., A 263351 (1988)

[6] F.Angelini, R.Bellazzini, A.Brez, G.Decarolis, C.Magazzu, M.M.Massai, G.Spandre and M.R.Torquati, Nucl. Instr. Meth., A 31521 (1992)

[7] F.Angelini et al., Proc. Large Hadron Collider Workshop, Aachen, Germany, CERN 90-10 pp.222 (1990)

[8] R.Bouclier et al., Development of microstrip gas chambers on thin plastic supports, CERN-PPE 91-108 (1991)

[9] A.R.Frolov et al., Nucl. Instr. Meth., A 307497 (1991)

[10] F.Sauli et al, RD-28 Status Report, CERN/DRDC/93-94, September 2, 1993

[11] G.D.Minakov, Y.N.Pestov, V.S.Prokopenko and L.I.Shekhtman, Nucl. Instr. Meth., A 326566 (1993)

[12] P.Savard, L-A.Hamel, J-P.Martin and P.Taras, Nucl. Instr. Meth., A 337387 (1993)

[13] W.S.Hong, G.Cho, V.Perez-Mendez and W.G.Gong, Presented at the MRS Spring Meeting, San Francisco, April 17-21, 1995 and to be published in the Proceedings

[14] Q.Wang, E.A.Schiff and Y.Li, Mat. Res. Soc. Symp. Proc., 297419 (1993)

[15] D.M.Bhusari, R.O.Dusane and S.T.Kshirsagar, J. Non-Cryst. Sol., 137\&138 689 (1991) 
[16] D.E.Anderson and W.E.Spear, Phil. Mag. B, 351 (1977)

[17] R.A.Street, Appl. Phys. Lett., 571334 (1990)

[18] J.Frenkel, Phys. Rev., 54647 (1938)

[19] R.Bouclier, C.Garabatos, G.Manzin, F.Sauli, L.Shekhtman, T.Temmel, G.Della Mea, G.Maggioni, V.Regato and I.Logachenko, IEEE Trans. Nucl. Sci., NS-41 821 (1994)

[20] S.Brons, W.Bruckner, M.Heidrich, I.Konorov and S.Paul, Nucl. Instr. Meth., A 342411 (1994)

[21] F.Angelini, R.Bellazzini, L.Bosisio, A.Brez, M.M.Massai, G.Spandre and M.R.Torquati, Nucl. Instr. Meth., A 314450 (1992)

[22] J.Bohm, R.Bouclier, M.Garabatos, G.Manzin, G.Million, F.Sauli, T.Temmel and L.Shekhtman, CERN-PPE/94-115, Presented at the 6th Pisa Conference "Frontier Detectors for Frontier Physics", Elba, May 24-28, 1994.

[23] R.Bouclier, M.Capeans, C.Garabatos, F.Sauli and K.Silander, CERN-PPE/94-111, Submitted to Nucl. Instr. Meth., 1994.

[24] R.Bouclier, C.Garabatos, G.Manzin, G.Million, F.Sauli, T.Temmel and L.shekhtman, CERN-PPE/94-63, Submitted to Nucl. Instr. Meth., 1994.

[25] F.Angelini, R.Bellazzini, A.Brez, M.M.Massai, R.Raffo, G.Spandre and M.A.Spezziga, Nucl. Instr. Meth., A 349412 (1994)

[26] F.Angelini, Presented at the IEEE Nucl. Sci. Symp., Norfolk, Oct. 31 - Nov. 6, 1994 


\section{CHAPTER 5. THIN $(<2 \mu \mathrm{m})$ a-Si:H LAYERS FOR READOUT AND OTHER APPLICATIONS}

\subsection{INTRODUCTION}

In many applications, the spatial distribution of the incident radiation is important. For example, a 2-dimensional array of detectors is required in applications such as tracking devices for high energy physics experiment, medical imaging, and synchrotron radiation transmission imaging for non-destructive examination.[1-3] Hence, pixel or strip configurations with appropriately shaped metallic contacts are used for position sensitive detectors. For other applications such as radiation flux detection, simple routing electronics may be sufficient.[4] In this configuration, detector pixels can be integrated with TFT or diode switches deposited by the same process and even read out by such TFT amplifiers. For $\mathrm{X}$-ray and $\gamma$-ray detection, thin a-Si:H p-i-n photodiodes can be coupled to $\mathrm{CsI}(\mathrm{Tl})$ scintillator layers of $100 \sim 1000 \mu \mathrm{m}$ in thickness with induced columnar structure for enhanced spatial resolution.[5]

Improved electrical transport properties are preferred over the other properties for the above applications. Higher carrier mobilities produce a faster response of the diodes and the TFTs. Lower ionized dangling bond density leads to smaller bias voltage sufficient for full depletion of detectors.

Hydrogen dilution of silane is known to improve photovoltaic characteristics in the a-Si:H solar cells, and sometimes results in formation of microcrystalline silicon ( $\mu \mathrm{c}-\mathrm{Si})$ depending on the deposition condition. Therefore, deposition of high-quality amorphous and microcrystalline silicon has been studied to obtain better electronic properties for integrated readout circuits and photodiode arrays. Material characteristics and microstructural study of the hydrogen-diluted material are discussed in section 5.2, and the applications of 2-dimensional amorphous silicon TFT arrays are described in section 5.3. 


\subsection{HYDROGEN DILUTION FOR BETTER ELECTRICAL TRANSPORT PROPERTIES}

\subsubsection{Introduction}

Since its first preparation through a chemical transport technique by Veprek et al. in 1968, microcrystalline silicon ( $\mu \mathrm{c}-\mathrm{Si}$ ) has been the subject of extensive research.[6] Due to its low optical absorption and high electrical conductivity compared to amorphous silicon (a-Si:H), p-doped $\mu \mathrm{c}-\mathrm{Si}$ has been used as window layers of solar cells.[7-9]. In addition, intrinsic and $\mathrm{n}^{+}$-doped microcrystalline silicon has been used as channel, source and drain contact material in thin film transistors (TFTs). The channel mobility of these devices is reported to be $\sim 6.5 \mathrm{~cm}^{2} / \mathrm{Vs}$, which is a factor of 5 higher than that of a-Si:H thin film transistors.[10]

By the PECVD technique it is possible to deposit microcrystalline material embedded in a-Si:H material with various degrees of crystallinity. Excess hydrogen in the plasma induces a transition to microcrystalline silicon. The free exchange of the hydrogen between the film and the plasma establishes equilibrium between the plasma gas and the film. Weak Si-Si bonds which lie below the hydrogen chemical potential are broken while stronger bonds remain. When hydrogen is added to the plasma, there is extensive reconstruction of the bonding at the growth surface, replacing weak bonds with strong ones. When the hydrogen chemical potential is low, it does not remove weak bonds and results in a more disordered structure. When the chemical potential is increased, the minimum disorder of an amorphous network contains too many weak bonds to be consistent with the chemical potential. The most ordered structure should occur when the hydrogen chemical potential is highest and this corresponds to the conditions for microcrystalline growth.[11] 
A variety of deposition conditions have been studied by different groups for their effects on deposition rate, volume fraction of crystallinity, and crystalline grain size.[12] Although there are many reports on electronic properties of doped $\mu \mathrm{c}-\mathrm{Si}$ films, they deal mostly with dark and photo conductivity and bandgap measurements [13-15]. On electronic transport properties such as carrier mobility and lifetime, especially for undoped $\mu \mathrm{c}-\mathrm{Si}$ layers, direct measurement results have been rarely available. In this section, the measurement results on both material and electronic properties are presented. Also, an analytical model explaining the relationship between the crystallinity and the observed transport characteristics is given.

\subsubsection{Experimental Procedures}

The samples used in this study were p-i-n diodes having $5 \sim 8 \mu \mathrm{m}$ thick i-layers, deposited in the PECVD machine at an RF frequency of $85 \mathrm{MHz}$. Corning 7059 glass plates, $0.8 \mathrm{~mm}$-thick, were sputtered with a semi-transparent $\mathrm{Cr}$ layer for electrical contact and were used as substrates. The thin $\mathrm{Cr}$ layer was also used as a top contact. Recipes for standard a-Si:H deposition were used in making the $300 \mathrm{~nm}$ thick $\mathrm{n}$ - and $\mathrm{p}$ - doped layers. Only the intrinsic layer was prepared with hydrogen dilution. The deposition conditions for different samples are given in Table V-1. The gas mixing ratio of hydrogen to silane, $\left[\mathrm{H}_{2}\right] /\left[\mathrm{SiH}_{4}\right]$ was varied between 10 and 20 , while the sum of $\mathrm{H}_{2}$ and $\mathrm{SiH}_{4}$ flow rates was kept at $100 \mathrm{sccm}$. All samples were deposited at the pressure of 1 Torr.

Drift mobility and mobility-lifetime product of charge carriers were measured by standard time-of flight and transient photocurrent technique, respectively, described in section 2.1.4. Curvature of the back surface of the substrate was measured by an Alphastep 200 profilometer, and the residual stress in the film was estimated from eqn. 
Intrinsic layers were deposited on glass substrates without a metal layer, and the $\mathrm{X}$ ray diffraction spectra were observed using a powder diffractometer. Very thin $(<1000 \AA)$ undoped layers were also deposited on glass, and were removed from the substrate by immersing in dilute HF or by scraping from the substrate. The flakes were collected on 300 mesh Cu grids and were observed in the Phillips 301 and Jeol ARM 100 Transmission Electron Microscope.(TEM) The crystallite sizes are measured either directly from TEM micrographs or from the XRD spectra using Scherer formula.[16]

Table V-1. Deposition conditions for the intrinsic layers of various runs

\begin{tabular}{|c|c|c|c|c|}
\hline Sample ID & {$\left[\mathrm{H}_{2}\right] /[\mathrm{SiH} 4]$} & $\mathrm{T}_{\mathrm{S}}\left({ }^{\circ} \mathrm{C}\right)$ & $\begin{array}{c}\text { Power Density } \\
\left(\mathrm{mW} / \mathrm{cm}^{2}\right)\end{array}$ & $\begin{array}{c}\text { Deposition Rate } \\
(\AA / \mathrm{sec}) \\
\end{array}$ \\
\hline MC388 & 10 & 190 & 60 & 2.8 \\
\hline MC392 & 10 & 190 & 90 & 4.1 \\
\hline MC389 & 10 & 250 & 60 & 3.7 \\
\hline MC361 & 15 & 190 & 60 & 4 \\
\hline MC362 & 15 & 190 & 90 & 4.3 \\
\hline MC214 & 15 & 250 & 30 & - \\
\hline MC363 & 15 & 250 & 60 & 3 \\
\hline MC292 & 20 & 190 & 60 & 2.2 \\
\hline MC370 & 20 & 190 & 90 & 2.1 \\
\hline MC200 & 20 & 250 & 30 & - \\
\hline MC354 & 20 & 250 & 60 & 2.2 \\
\hline MC190 & 20 & 320 & 30 & - \\
\hline MC257 & 30 & 190 & 30 & - \\
\hline $\mathrm{MC} 256$ & 30 & 190 & 60 & - \\
\hline $\mathrm{MC} 255$ & 30 & 190 & 90 & - \\
\hline standard a-Si:H & 0 & 250 & 50 & 7 \\
\hline
\end{tabular}




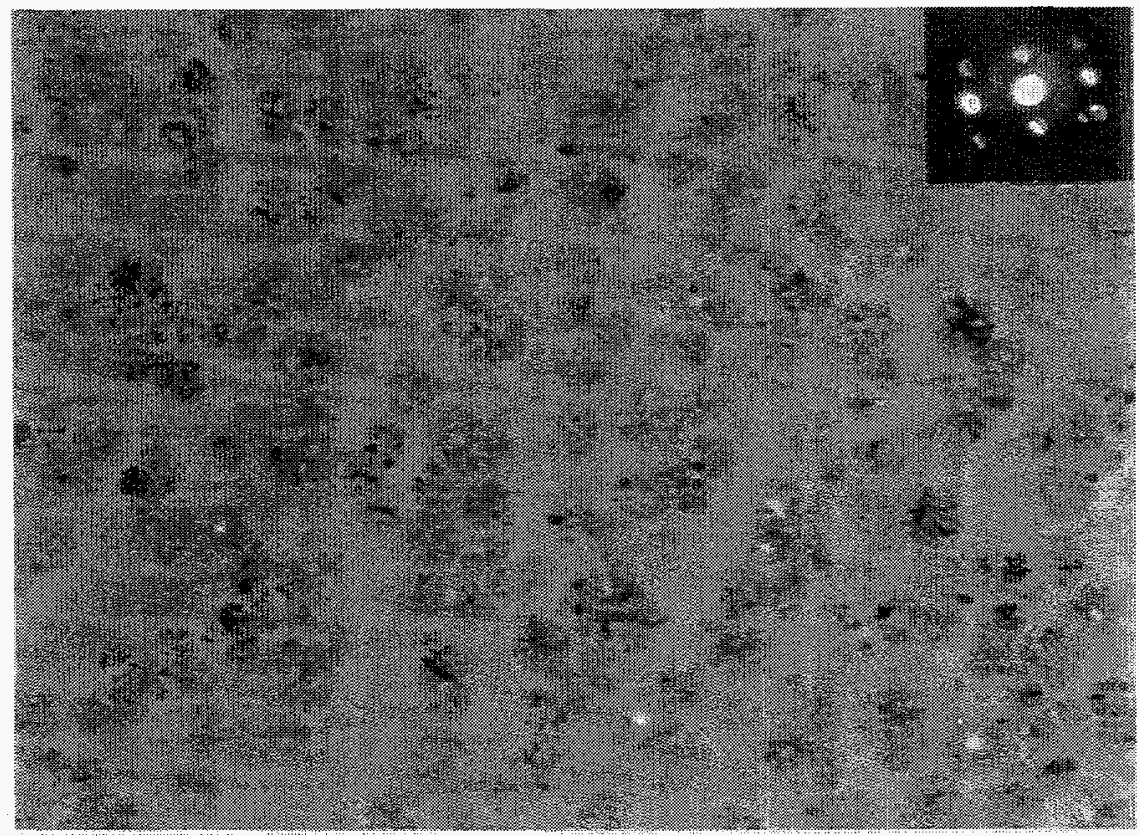

$250 \mathrm{~nm}$

Figure 5.1. A TEM bright-field image and the associated micro-diffraction pattern of a hydrogen-diluted sample prepared at $\mathrm{H}_{2} / \mathrm{SiH}_{4}$ ratio of 20 , substrate temperature of $190^{\circ} \mathrm{C}$ and power density of $90 \mathrm{~mW} / \mathrm{cm}^{2}$.

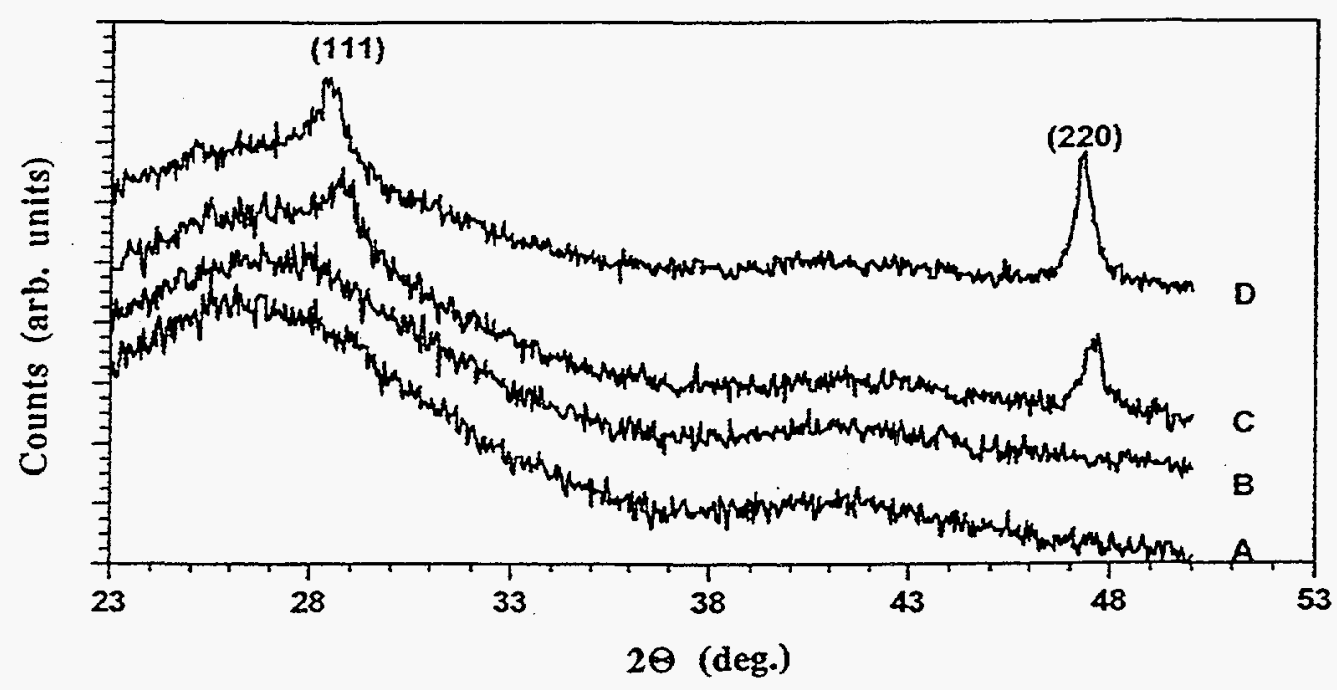

Figure 5.2. X-ray diffraction patterns for samples prepared at four different conditions. The RF power density and gas mixing ratio are $60 \mathrm{~mW} / \mathrm{cm} 2$ and 10 for sample $A, 60 \mathrm{~mW} / \mathrm{cm}^{2}$ and 15 for sample $B, 60 \mathrm{~mW} / \mathrm{cm}^{2}$ and 20 for sample $C, 90 \mathrm{~mW} / \mathrm{cm}^{2}$ and 20 for sample $D$, respectively. All samples were deposited at a substrate temperature of $190^{\circ} \mathrm{C}$. 


\subsubsection{Results}

Figure 5.1 shows a TEM image taken from a sample deposited at a hydrogen to silane mixing ratio of 20 , a substrate temperature of $190^{\circ} \mathrm{C}$ and a power density of 60 $\mathrm{mW} / \mathrm{cm}^{2}$. Inset is an electron micro-diffraction pattern from a crystallite. X-ray diffraction patterns for 4 different samples are shown in Fig. 5.2. The microcrystalline structure was clearly revealed by TEM images and was also confirmed by XRD spectra. Both TEM images and micro diffraction patterns showed Si microcrystals embedded in a-Si:H matrix.

In the $\mathrm{X}$-ray diffraction method, the crystallite size is estimated by measuring the peak broadening due to the insufficient number of lattice planes to build up a sharp diffraction peak. The full-width-at-half-maximum(FWHM), $\beta$, of a characteristic peak is related to the crystallite size by the Scherer formula[16]:

$$
D=\left(\frac{\ln 2}{\pi}\right)^{1 / 2} \frac{1}{\beta \cos \theta}
$$

where $D$ is the average dimension of the crystallites and $\theta$ is the Bragg angle. According to Warren, the peak broadening due purely to the size effect can be obtained as follows[17]:

$$
\beta=\left[\left(\beta_{\text {meas }}-\beta_{\text {instr }}\right) \sqrt{\beta_{\text {meas }}^{2}-\beta_{\text {insir }}^{2}}\right]^{1 / 2}
$$

where $\beta_{\text {meas }}$ is the measured FWHM from the spectrum and $\beta_{\text {instr }}$ is the FWHM in the spectrum of a standard, e.g., single crystal sample. However, the crystallite sizes measured in this way are smaller by $1 \sim 2$ orders of magnitude than those from the TEM pictures. It is seen from the atomic-resolution TEM picture in Fig. 5.3 that each particle consists of tiny sub-grains of the order of $10^{3} \sim 10^{5}$ atoms. The crystallite dimension measured from the XRD spectra represents the average size of these sub-grains whereas the ordinary TEM pictures only shows the clusters of these sub-grains as blobs. This explains the discrepancy between the estimated crystallite sizes from the XRD and the TEM. 


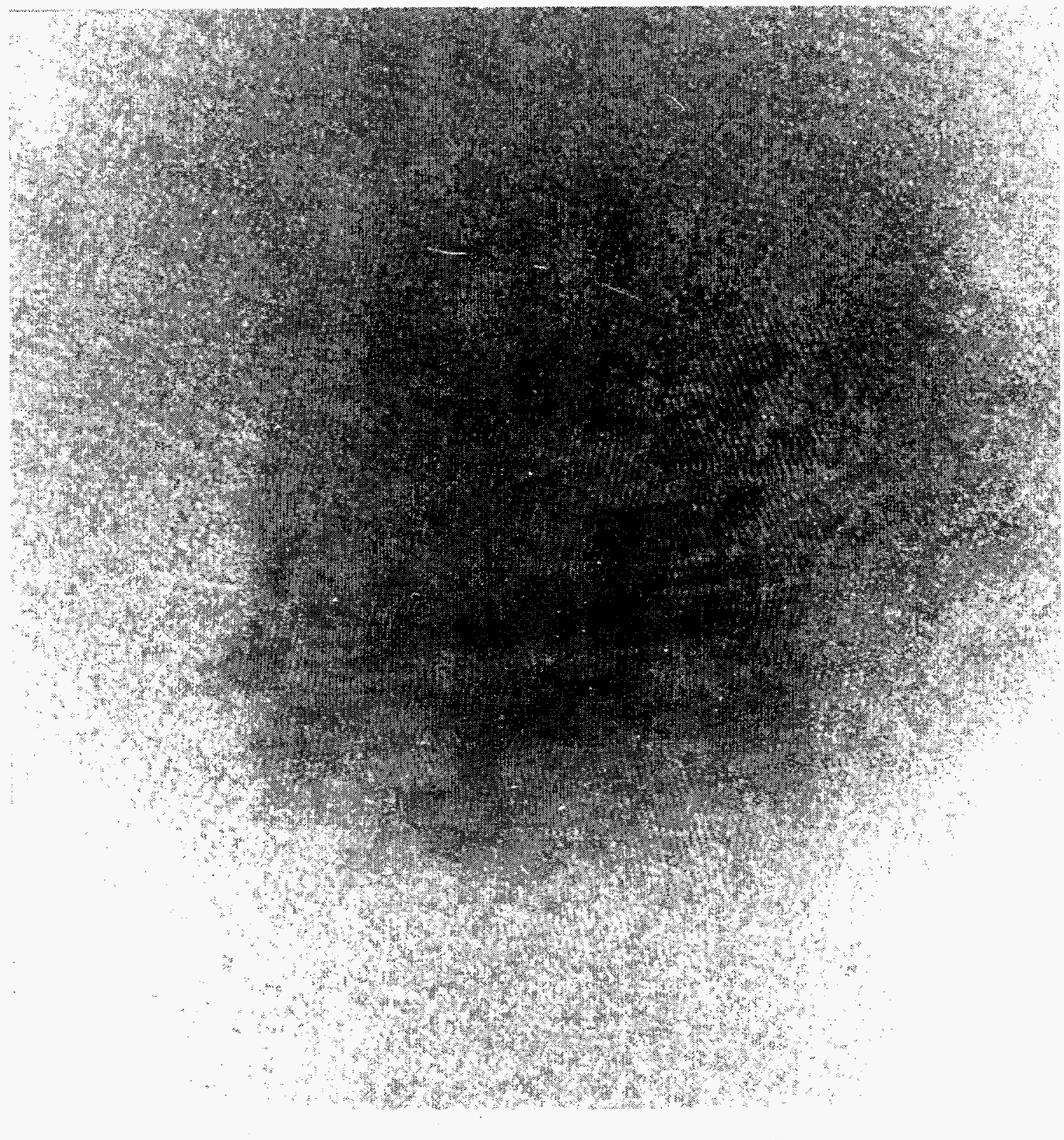

$5 \cdot \mathrm{nm}$

Figure 5.3. Atomic resolution TEM image showing crystalline lattice planes 


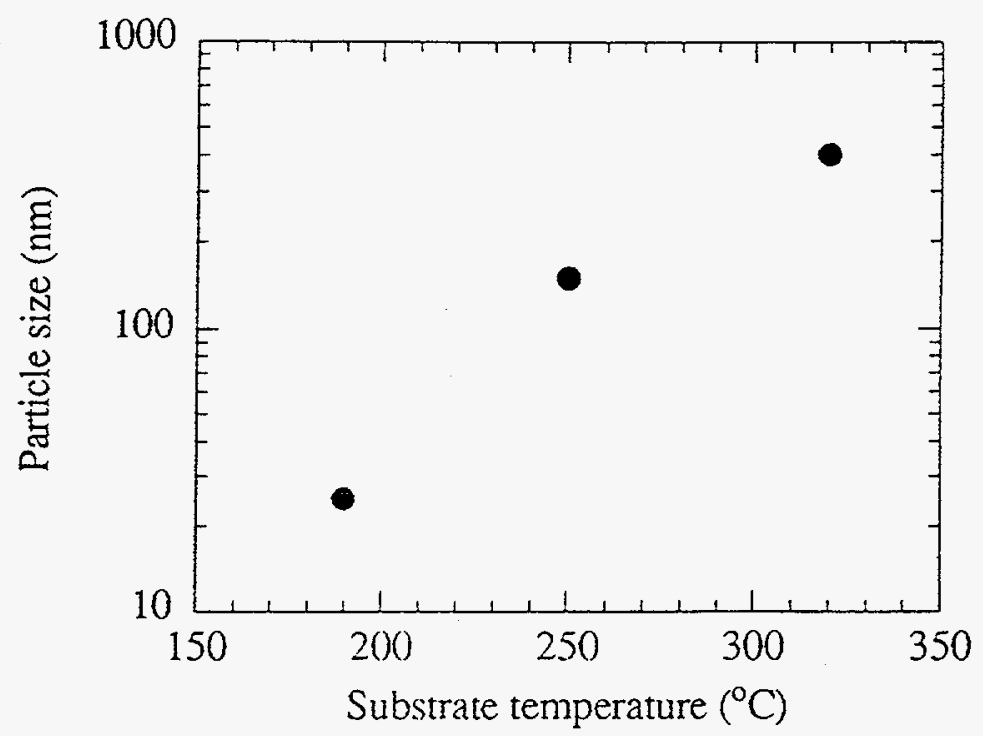

(a)

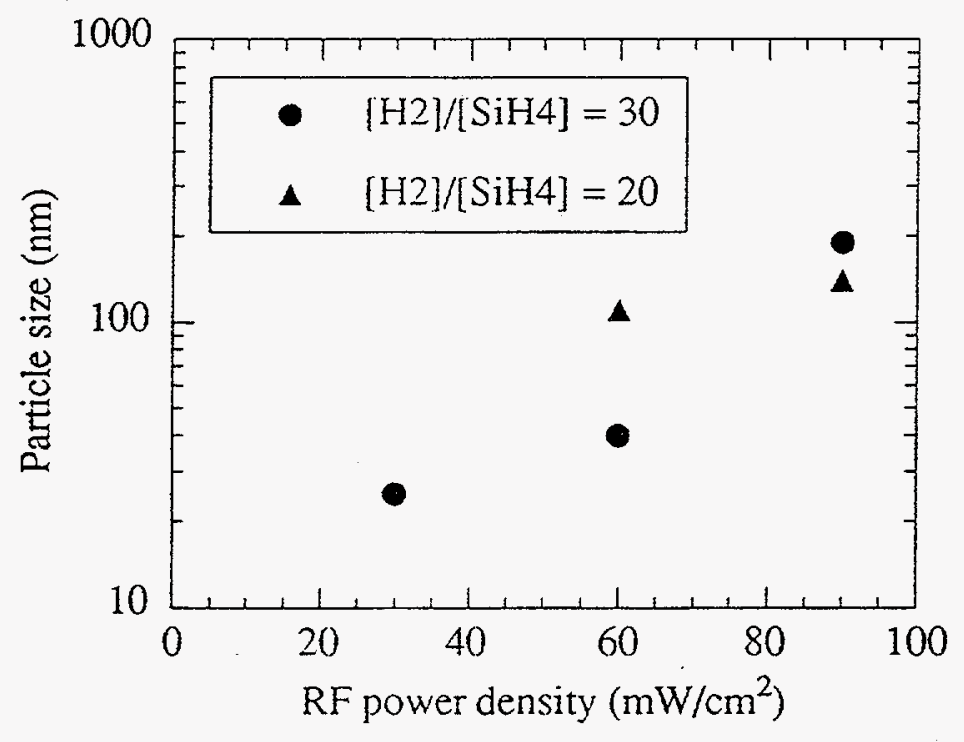

(b)

Figure 5.4. Change in particle size as a function of (a) substrate temperature, and (b) RF power density 


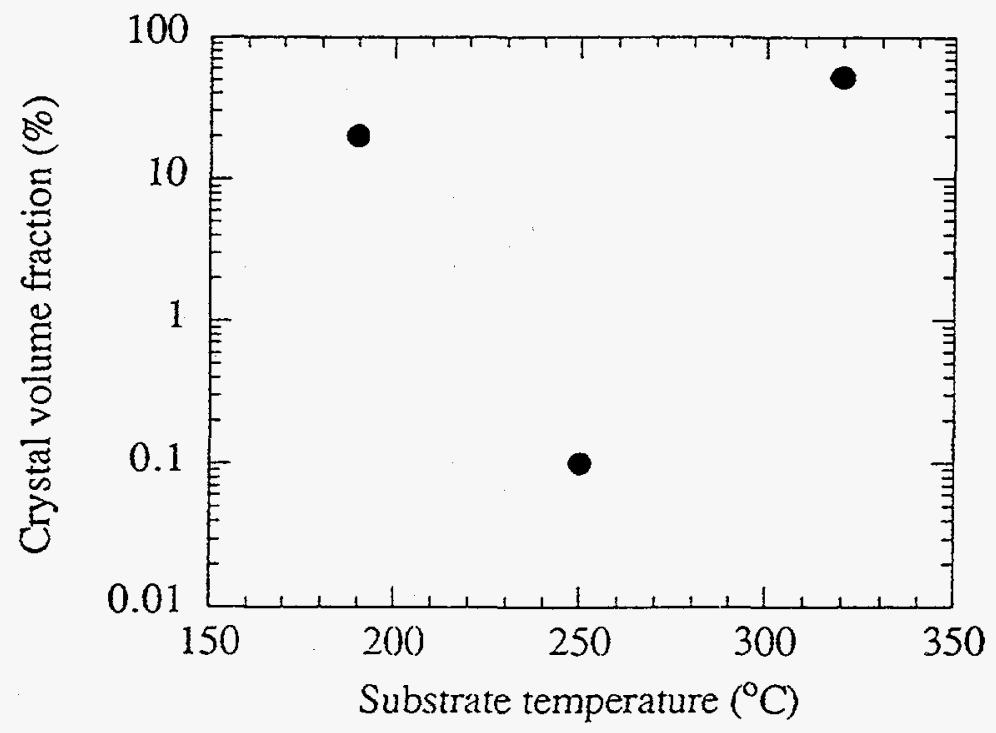

(a)

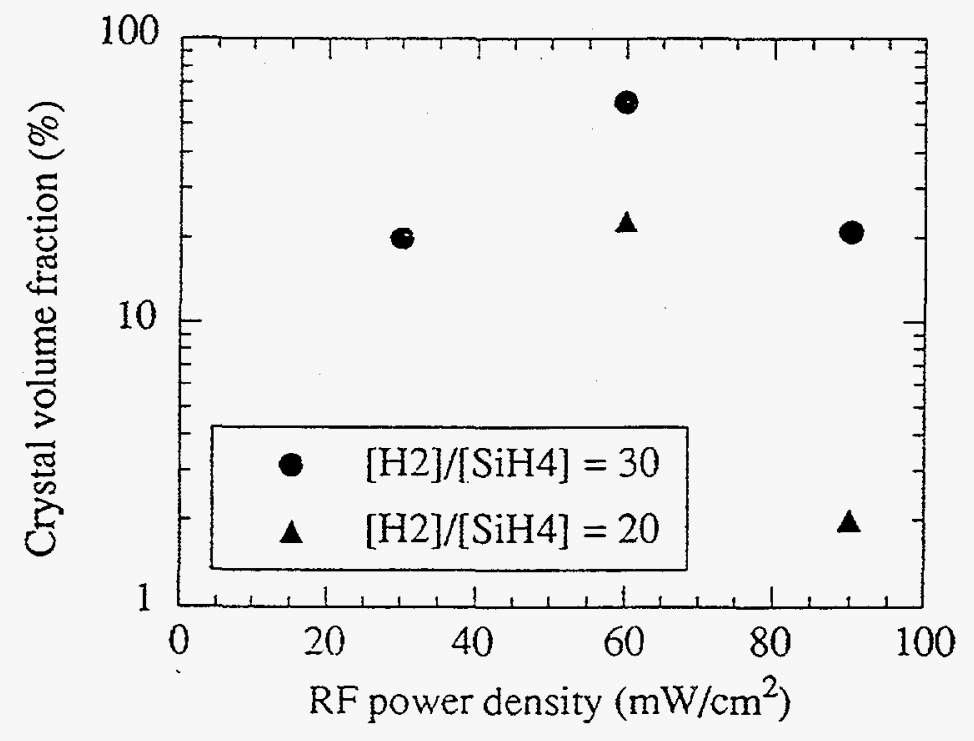

(b)

Figure 5.5. Change in crystalline volume fraction as a function of (a) substrate temperature, and (b) RF power density 
The degree of crystallinity and the grain size depends on the deposition conditions. The grain sizes and crystallinity values determined from the TEM are consistent with other reports.[12] At a hydrogen to silane ratio of 10 , no trace of crystals was found. At a dilution ratio of 15 , there was a large sample-to-sample variation, but in general, little or no crystallinity was observed. A noticeable amount of crystallinity was observed at gas mixing ratios higher than 15 . Figure 5.4 shows variations in particle size as a function of deposition temperature and RF power density. As shown in Fig. 5.4(a), for the gas mixing ratio of 20 and the $R F$ power density of $90 \mathrm{~mW} / \mathrm{cm}^{2}$, the particle size increased from $25 \mathrm{~nm}$ to $400 \mathrm{~nm}$ with increasing substrate temperature from $190^{\circ} \mathrm{C}$ to $320^{\circ} \mathrm{C}$. In Fig. 5.4(b), when the deposition temperature is $190^{\circ} \mathrm{C}$, the particle size increased from $25 \mathrm{~nm}$ to $190 \mathrm{~nm}$ as the RF power density increased from $30 \mathrm{~mW} / \mathrm{cm}^{2}$ to $90 \mathrm{~mW} / \mathrm{cm}^{2}$ for the gas mixing ratio of 30 . On the other hand, for the hydrogen/silane ratio of 20 , increasing the power density from $60 \mathrm{~mW} / \mathrm{cm}^{2}$ to $90 \mathrm{~mW} / \mathrm{cm}^{2}$ increased the particle size only by $25 \%$.

As seen from Fig. 5.5, the crystalline volume fraction did not show a systematic trend either with the deposition temperature or with the RF power density. Nevertheless, it can be said that the volume fraction increased as the dilution ratio increased. The increase in particle size with RF power or with substrate temperature and the non-systematic behavior of the crystalline fraction can be qualitatively explained by homogeneous nucleation and growth theory in solidification of supercooled liquids.[18]

The particle size depends solely on the growth rate, whereas the overall crystallinity depends more on the number of particles which is in turn influenced by nucleation rate. The growth of a crystalline phase out of an amorphous matrix is usually a diffusion controlled process and its rate increases gradually with the driving force for the atoms to move to specific sites. Therefore, the growth is expedited when more energy is transferred to the atoms in the form of RF power or heat. On the contrary, the nucleation shows somewhat unpredictable behavior with the driving force, e.g., temperature. Regarding the amorphous phase as supercooled liquid, the homogeneous nucleation rate, $N$, is given by 


$$
N=f_{o} C_{o} \exp \left(-\frac{A}{(\Delta T)^{2}}\right) \quad\left[\text { nuclei } / \mathrm{m}^{3} \cdot \mathrm{sec}\right]
$$

where $f_{o}$ is the vibration frequency of the atoms, $C_{o}$ is the number of atoms per unit volume of amorphous matrix, $\Delta T$ is the degree of supercooling. $A$ is relatively insensitive to temperature and is given by

$$
A=\frac{16 \pi \gamma_{S L}^{3} T_{m}^{2}}{3 L_{v} k T}
$$

where $\gamma_{S L}$ is the solid/liquid interfacial free energy, $T_{m}$ is the meting point of silicon, $L_{v}$ is the latent heat of fusion per unit volume. When $N$ is plotted as a function of $\Delta T$, inside the exponential $N$ changes by orders of magnitude from essentially zero to very high values over a very narrow temperature change as a result of the $(\Delta T)^{2}$ term. In other words, there is effectively a critical supercooling for nucleation $\Delta T_{N}$. Virtually no nuclei are formed until $\Delta T_{N}$ is reached after which there is an 'explosion' of nuclei. This explains why the crystalline volume fraction showed an abrupt change with substrate temperature. Similarly, the energy provided to atoms by the RF power is believed to change the effective $\Delta T$ and influence the volume fraction in the same way. However, the amount of extra hydrogen is connected to the local strain energy and to the interface energy, and thus only affects the temperature-insensitive parameter $A$. Hence, at the same temperature and RF power density, the crystallinity undergoes relatively more systematic change with the hydrogen dilution ratio.

In the XRD spectra of samples $C$ and $D$,(see Fig. 5.2) a prominent peak at $2 \theta=$ $47.3^{\circ}$ corresponds to the (220) orientation, and renders an idea that some specific texture with the ordered structure was constructed in the film. The same strong (220) orientation has also been reported by other researchers for the $\mu \mathrm{c}-\mathrm{Si}: \mathrm{H}$ films prepared by a chemical annealing method.[19] 


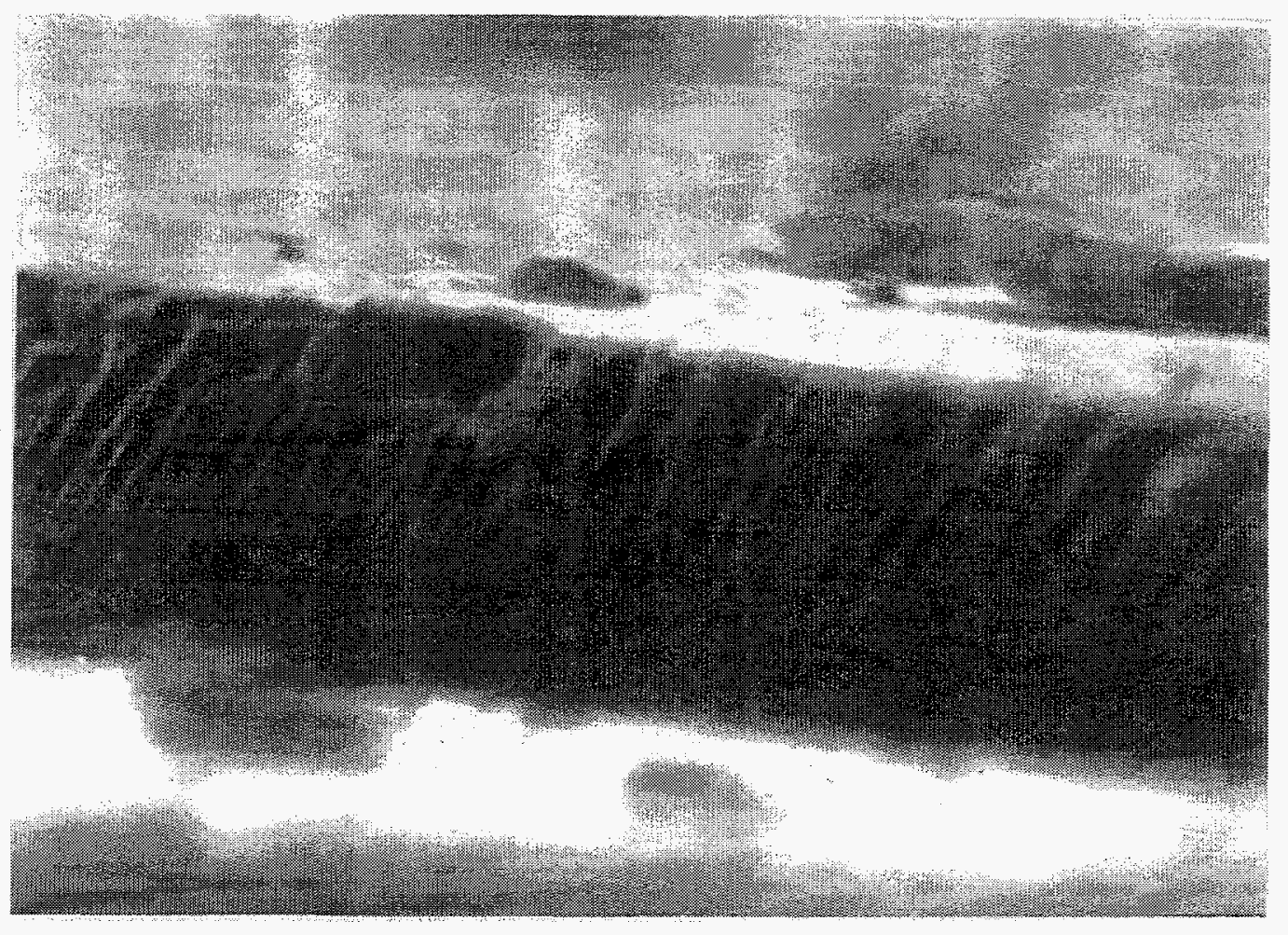

$$
0.5 \mu \mathrm{m}
$$

Figure 5.6. SEM micrograph of a fractured cross-section of sample MC292. 
I-V characteristics of the $\mathrm{p}-\mathrm{i}-\mathrm{n}$ diodes having the $\mathrm{H}$-diluted $\mathrm{i}$-layers were also measured. The reverse currents were generally one or two orders of magnitude higher than that of the best standard a-Si:H diode. The higher the hydrogen dilution, the larger the leakage current. For hydrogen to silane ratio of 30 , samples showed the forward-to reverse current ratio too low to be used as diodes. It seems that at this level of hydrogen dilution the material has voids and inhomogeneous microstructure and that the junctions formed at the $\mathrm{p}-\mathrm{i}$ and $\mathrm{n}-\mathrm{i}$ interfaces do not effectively block the reverse current.

Some samples were inspected with a Scanning Electron Microscope to probe the morphology. The films were free from microscopic defects, such as columnar structure or voids, up to a gas mixing ratio of 20 . However, for the samples prepared with dilution ratio of 30 , the surface was indeed not shiny and the cross sectional SEM revealed columnar morphology.(see Fig. 5.6) This explains why the diodes made with the mixing ratio of 30 have enormous leakage current. It should be noted that samples which functioned reasonable well as diodes had crystalline fractions less than $1 \%$ and particle sizes smaller than $100 \mathrm{~nm}$.

Table V-2 Measured electron and hole transport parameters and stress values

\begin{tabular}{ccccccc}
\hline Sample & $\begin{array}{c}\mu_{\mathrm{e}} \\
\left(\mathrm{cm}^{2} / \mathrm{V} \cdot \mathrm{s}\right)\end{array}$ & $\begin{array}{c}(\mu \tau)_{\mathrm{e}} \\
\left(\mathrm{cm}^{2} / \mathrm{V}\right)\end{array}$ & $\begin{array}{c}\mu_{\mathrm{h}} \\
\left(\mathrm{cm}^{2} / \mathrm{N} \cdot \mathrm{s}\right)\end{array}$ & $\begin{array}{c}(\mu \tau)_{\mathrm{h}} \\
\left(\mathrm{cm}^{2} / \mathrm{V}\right)\end{array}$ & $\begin{array}{c}\mathrm{N}_{\mathrm{d}}{ }^{*} \\
\left(\mathrm{~cm}^{-3}\right)\end{array}$ & $\begin{array}{c}\text { Stress } \\
(\mathrm{MPa})\end{array}$ \\
\hline MC361 & 4.2 & $2.2 \times 10^{-7}$ & 0.013 & $6.0 \times 10^{-8}$ & $2 \times 10^{13}$ & 650 \\
$\mathrm{MC} 362$ & 2.7 & $1.1 \times 10^{-7}$ & 0.009 & $3.4 \times 10^{-8}$ & $9 \times 10^{13}$ & 680 \\
$\mathrm{MC} 363$ & 1.2 & $1.2 \times 10^{-7}$ & 0.006 & $1.7 \times 10^{-8}$ & $3 \times 10^{13}$ & 610 \\
MC292 & 2.5 & $3.0 \times 10^{-7}$ & 0.011 & $1.7 \times 10^{-8}$ & $1.6 \times 10^{14}$ & 650 \\
MC370 & 1 & $1.4 \times 10^{-7}$ & 0.009 & $2.0 \times 10^{-8}$ & $1.4 \times 10^{14}$ & 580 \\
MC354 & 1.1 & $1.5 \times 10^{-7}$ & 0.003 & $2.0 \times 10^{-8}$ & $1.3 \times 10^{14}$ & 720 \\
Std. A-Si:H & 1.2 & $1.2 \times 10^{-7}$ & 0.004 & $2.6 \times 10^{-8}$ & $7 \times 10^{14}$ & 350 \\
\hline
\end{tabular}




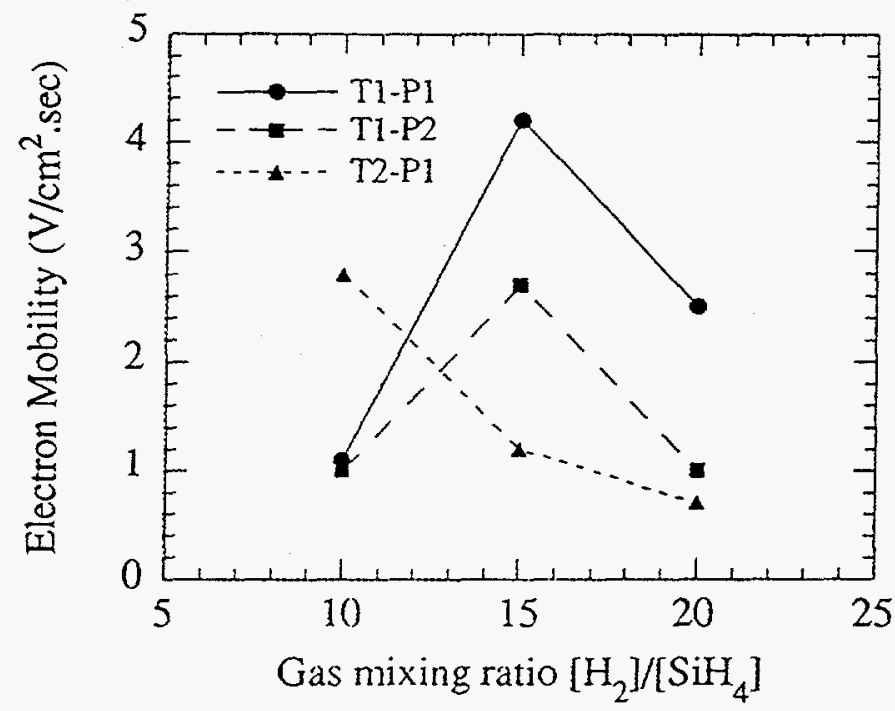

(a)

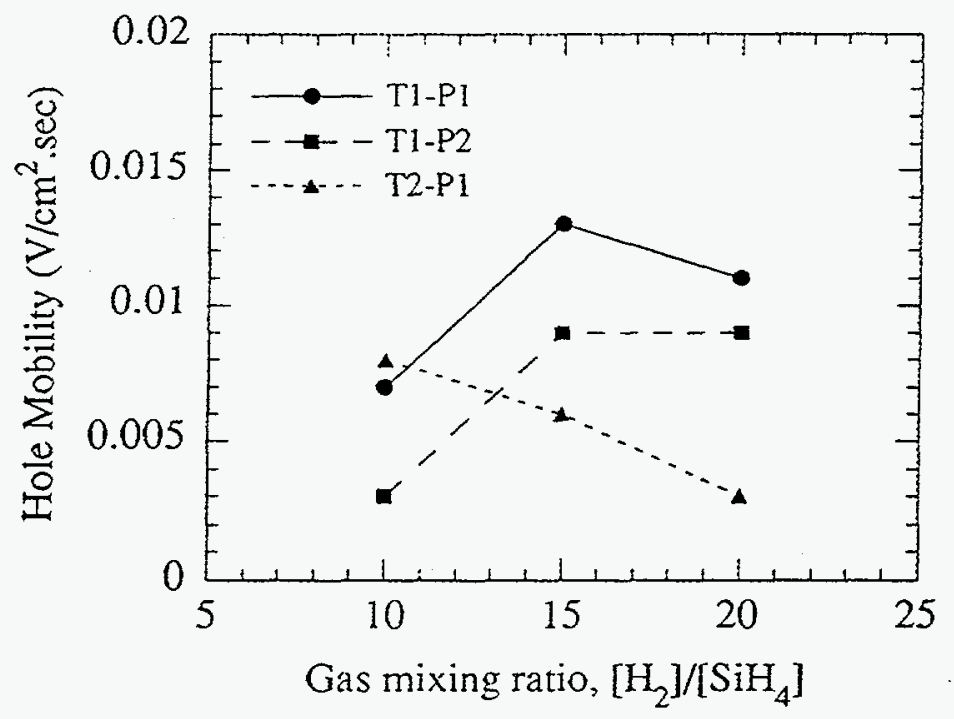

(b)

Figure 5.7. Changes in drift mobilities of (a) electron, and (b)hole as functions of the gas mixing ratio, $\left[\mathrm{H}_{2}\right] /\left[\mathrm{SiH}_{4}\right]$ for three sets of deposition conditions. $T_{1}$ and $T_{2}$ represent substrate temperatures of 190 and $250^{\circ} \mathrm{C}$, respectively, and $P_{1}$ and $P_{2}$ refer to $R F$ power densities of 60 and 90 $\mathrm{mW} / \mathrm{cm}^{2}$, respectively. 


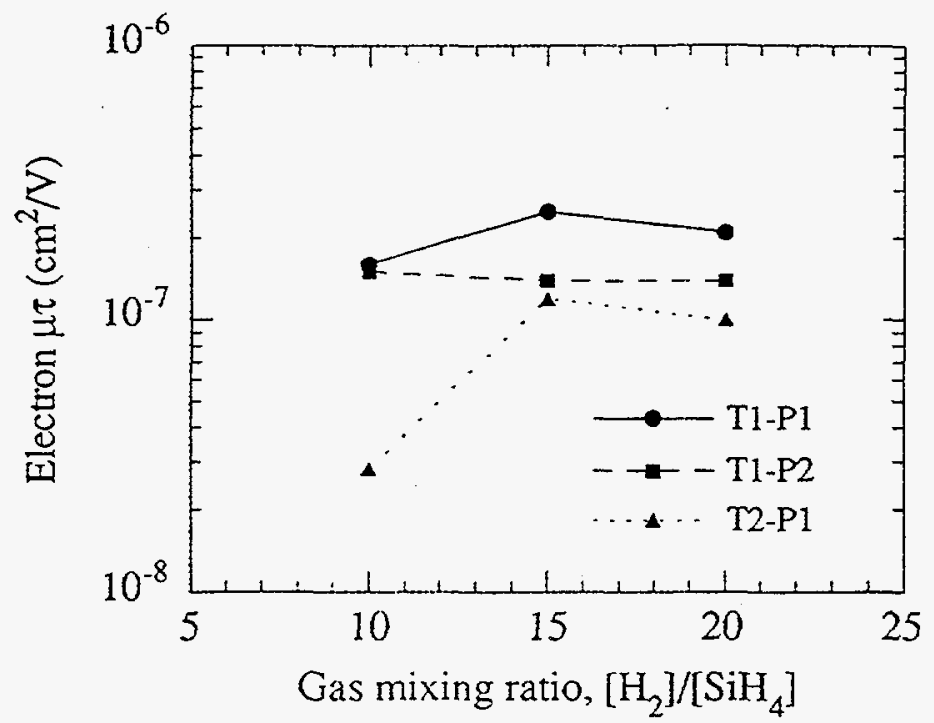

(a)

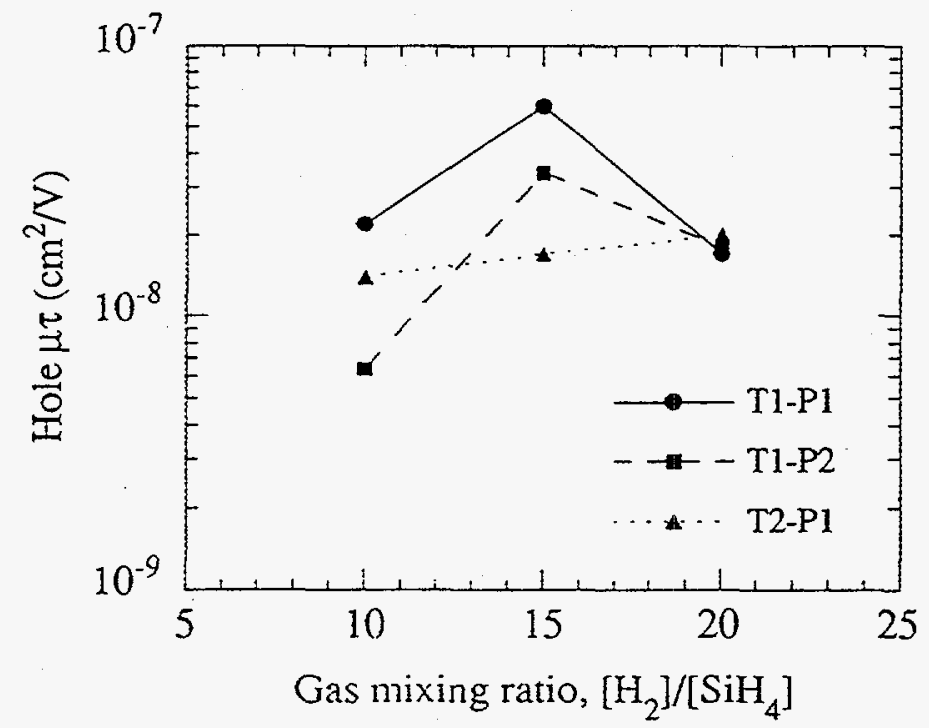

(b)

Figure 5.8. Variations of mobility-lifetime product values of (a) electron, and (b)hole as functions of the gas mixing ratio, $\left[\mathrm{H}_{2}\right] /\left[\mathrm{SiH}_{4}\right]$ for three sets of deposition conditions. $T_{1}$ and $T_{2}$ represent substrate temperatures of 190 and $250^{\circ} \mathrm{C}$, respectively, and $P_{1}$ and $P_{2}$ refer to RF power densities of 60 and $90 \mathrm{~mW} / \mathrm{cm}^{2}$, respectively. 
Table V-2 gives the results of electron and hole mobility and $\mu \tau$, as well as $N_{d}{ }^{*}$, for samples deposited at various conditions. The results from the $\mathrm{H}$-dilution ratio of 30 are absent because they had reverse current so large that any measurement requiring reversebias could not be performed. The last row gives the parameters of standard a-Si:H material. The measured electron and hole mobility values for these hydrogen-diluted samples depend largely on the deposition condition. In Fig. 5.7, changes in electron and hole mobilities as a function of the gas mixing ratio, $\left[\mathrm{H}_{2}\right] /\left[\mathrm{SiH}_{4}\right]$, for three sets of deposition parameters are plotted. In Fig. 5.8, changes in mobility-lifetime product as a function of the gas mixing ratio are plotted for electron and hole. The mobilities have a maximum value at a hydrogen to silane ratio of 15 , for both electrons and holes, except for the samples deposited at substrate temperature, $T_{s}=250^{\circ} \mathrm{C}$ and power density, $P=60$ $\mathrm{mW} / \mathrm{cm}^{2}$, for which the mobility drops with increasing hydrogen dilution. The samples deposited at $T_{s}=190^{\circ} \mathrm{C}$ and $P=60 \mathrm{~mW} / \mathrm{cm}^{2}$ show the highest electron and hole mobilities, a factor of about 4 and 3 higher than the normal a-Si:H values, respectively. For the same sample (MC361) the $\mu \tau$ values for holes and electrons have improved approximately by a factor of 2, compared to conventional a-Si:H. These effects either decreased or disappeared when higher temperature or higher power was used. At a hydrogen to silane ratio of 20 , the sample produced at $190^{\circ} \mathrm{C}$ and $60 \mathrm{~mW} / \mathrm{cm}^{2}$ improved overall characteristics for electrons, while for holes, only the mobility increased. The hole mobility for all samples deposited at lower temperature of $190^{\circ} \mathrm{C}$ showed an improvement by a factor of 2 to 3 over the standard a-Si:H values. For the electron $\mu \tau$, the values for higher RF power (dashed lines) samples, showed no variation with hydrogen ratio, while for the holes the higher temperature ones showed similar behavior. 


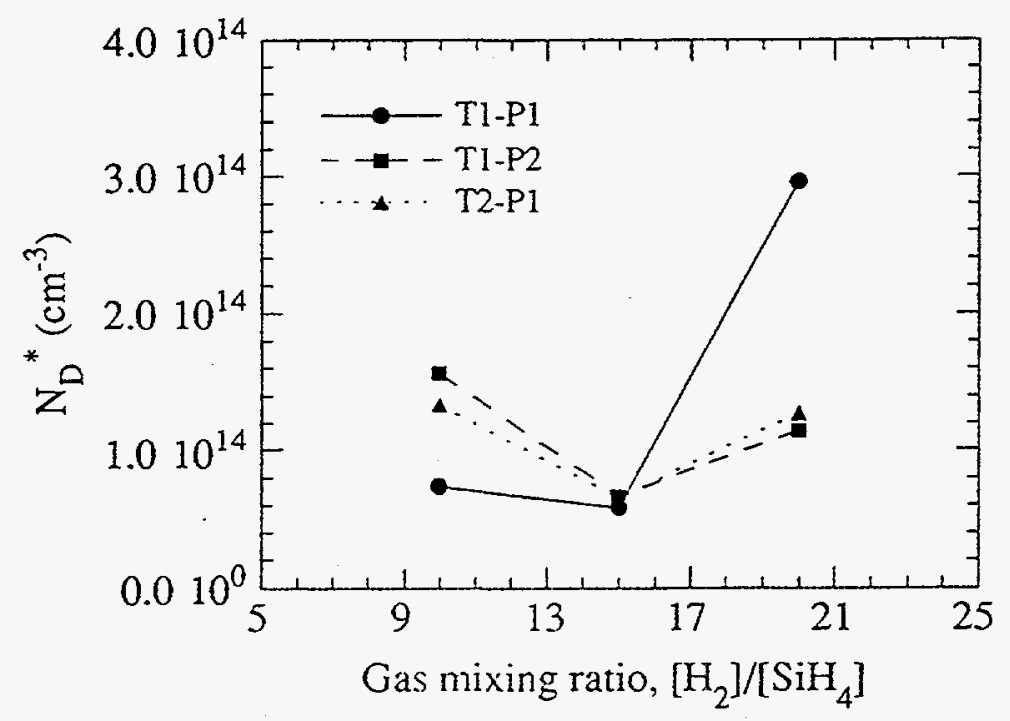

Figure 5.9. The effect of hydrogen dilution on the ionized defect density for samples prepared at three different conditions. $T_{1}$ and $T_{2}$ represent substrate temperatures of 190 and $250^{\circ} \mathrm{C}$, respectively, and $P_{1}$ and $P_{2}$ refer to RF power densities of 60 and $90 \mathrm{~mW} / \mathrm{cm}^{2}$, respectively.

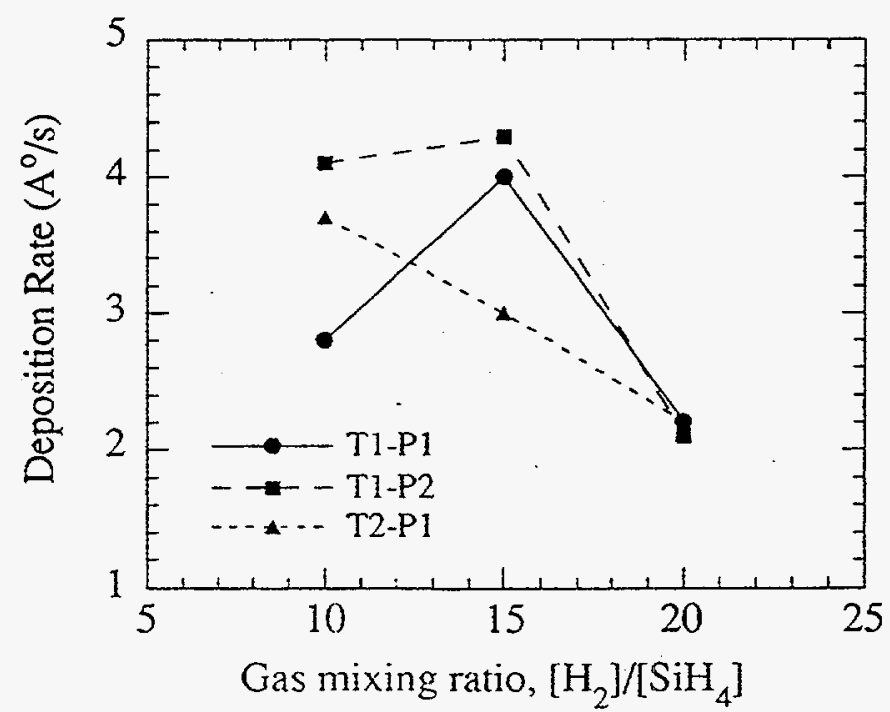

Figure 5.10. Effect of hydrogen dilution on the deposition rate for samples prepared at three different conditions. $T_{1}$ and $T_{2}$ represent substrate temperatures of 190 and $250^{\circ} \mathrm{C}$, respectively, and $P_{1}$ and $P_{2}$ refer to $R F$ power densities of 60 and $90 \mathrm{~mW} / \mathrm{cm}^{2}$, respectively. 
The $N_{d}{ }^{*}$ values for hydrogen diluted samples derived from hole onset measurement are in general lower than that of standard a-Si:H. The built-in potential was assumed to be 0.9 volts and applied to calculate hole onset voltage. The change in ionized dangling bond densities for various deposition conditions is given in Fig. 5.9. The $N_{d}{ }^{*}$ values are lowest at a gas mixing ratio of 15 , for all three sets of deposition conditions. These values are considerably lower than the value of $7 \times 10^{14} \mathrm{~cm}^{-3}$ that we normally obtain in standard aSi:H samples.

Deposition rates of various samples are plotted against the gas mixing ratio in Fig. 5.10. The use of higher power resulted in larger deposition rates, except for the hydrogen ratio of 20 , where the rate is not sensitive to the preparation condition. At $T_{S}=250^{\circ} \mathrm{C}$, the deposition rate decreases with increasing hydrogen dilution. In general the deposition rates are about $1 / 2$ to $1 / 3$ of that of the standard a-Si:H at this RF frequency ( $85 \mathrm{MHz}$ ).

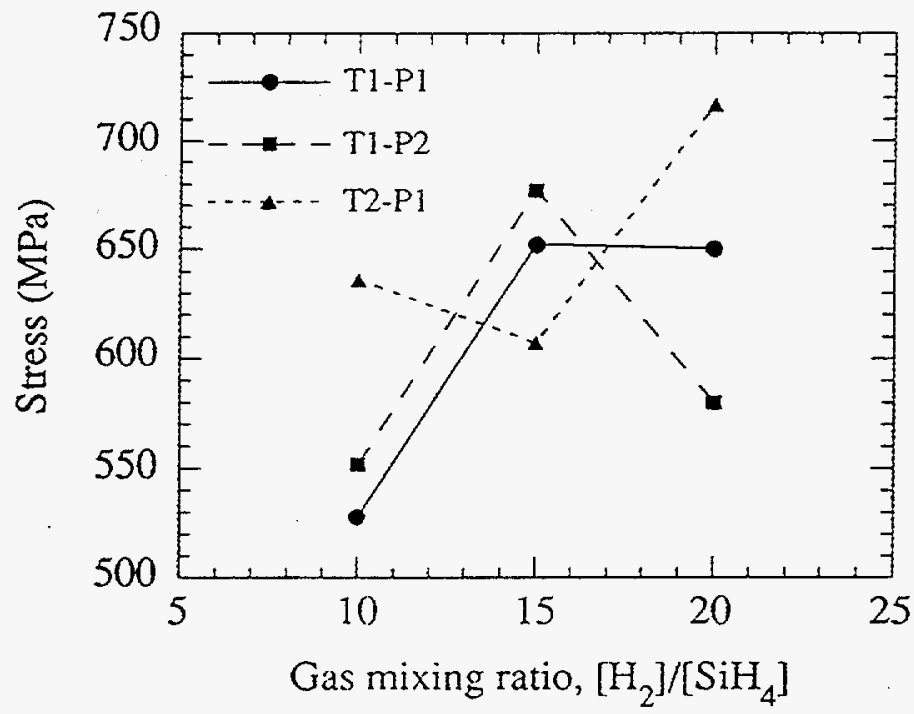

Figure 5.11 Effect of hydrogen dilution on the film stress for samples prepared at three different conditions. $T_{1}$ and $T_{2}$ represent substrate temperatures of 190 and $250^{\circ} \mathrm{C}$, respectively, and $\mathrm{P}_{1}$ and $\mathrm{P}_{2}$ refer to $\mathrm{RF}$ power densities of 60 and $90 \mathrm{~mW} / \mathrm{cm}^{2}$, respectively. 
The results of stress measurement are shown in Fig. 5.11. The measured stress values are about 1.5 to 2 times greater than that of standard a-Si:H. The increase in the residual stress with hydrogen dilution is also observed by others.[20] For samples prepared at $T_{s}=190^{\circ} \mathrm{C}$, the maximum stress appeared at the deposition parameters that produce the lowest $N_{d}{ }^{*}$. The high stess in the hydrogen diluted material hinders it from being used in thick layer or large area applications.

\subsubsection{Discussion}

Based on the experimental results given in figures $5.7 \sim 5.11$, an optimum deposition condition is determined to be hydrogen to silane ratio $=15$, substrate temperature $=190^{\circ} \mathrm{C}$, and $R F$ power density $=60 \mathrm{~mW} / \mathrm{cm}^{2}$. At this condition, almost all electronic transport characteristics have their maximum values. The electron and hole mobilities are about 3-4 times larger than those of standard a-Si:H. The increase in hole mobilities has also been reported by the Dundee group for their hydrogen-diluted samples deposited by microwave decomposition from a $\mathrm{H}_{2}-\mathrm{SiF}_{4}$ mixture.[15]

The presence of a crystalline phase is expected to affect the electrical transport. Drift mobility can be increased because of the narrowed band tail. Carrier lifetime may be reduced due to capture at the amorphous-crystalline phase boundary or grain boundaries inside crystallites. Analytical modeling is attempted to forecast the change in mobility and lifetime as a function of crystallinity of the material.

\section{(1) Carrier mobility}

For simplicity, the hydrogen-diluted material is modeled as spherical singlecrystalline particles with an average diameter, $D$, embedded in a continuous amorphous network. The crystalline and amorphous phases are assumed to be separated by a phase 
boundary of thickness $d$. In a multiphase material, the average carrier mobility, $\mu$, can be expressed in terms of the volume fraction of each phase $X$.

$$
\frac{1}{\mu}=\sum_{i} \frac{X_{i}}{\mu_{i}}=\frac{X_{a}}{\mu_{a}}+\frac{X_{b}}{\mu_{b}}+\frac{X_{c}}{\mu_{c}}
$$

where subscripts $a, b$, and $c$ represent amorphous, phase boundary, and crystalline region, respectively. From the geometry, with $d \ll D$, the volume fraction of phase boundaries is expressed as $X_{b}=6 d X_{c} / D$. Also, since $X_{b}$ is very small, $X_{a}$ is approximated to $1-X_{c}$. Then rewriting $X_{a}$ and $X_{b}$ in terms of $X_{c}$ and rearranging eqn. (5.2.5) lead to

$$
\frac{\mu}{\mu_{a}}=\frac{1}{1-X_{d}\left(1-\frac{6 d}{D} K\right)}
$$

with $K=\mu_{a} / \mu_{b}$. Therefore, the ratio of the macroscopically observed average mobility to the mobility in the amorphous matrix can be described as a function of the volume fraction of the crystalline phase. If we adopt a typical value of $\mu_{a}=1 \mathrm{~cm}^{2} / \mathrm{V} \cdot \mathrm{sec}$ and assume that $\mu_{a}$ does not change with volume fraction, eqn. (5.2.6) can be used directly to estimate the average mobility for given parameters. In Fig. 5.12, the parameter $\mu / \mu_{a}$ is calculated for five different values of $K$ and is plotted against the crystalline volume fraction. An average crystallite diameter of $55 \mathrm{~nm}$ and a phase boundary thickness of $0.5 \mathrm{~nm}$ were used in the calculation. We see from the plot that until the crystalline fraction reaches $\sim 30 \%$, the overall mobility would not show any noticeable change. Although not shown in the plot, calculation shows that the mobility increases drastically beyond the crystalline fraction of $90 \%$ and approaches the values of polycrystalline silicon. In the low crystallinity regime $\left(X_{c}<30 \%\right.$ ), one can imagine easily that, unless the crystallites touch one another, even though the mobility inside the crystals would be infinite, the overall mobility will be 
governed by that of the amorphous matrix. Once the crystalline particles are touching, carriers can find a short-cut and the mobility is close to that of polysilicon.

However, experimental results conflict with this model prediction. For the samples prepared at $T_{s}=190^{\circ} \mathrm{C}$ from gas mixing ratio of 15 , the crystallinity is less than $10 \%$, but measured mobility values showed a factor of $2 \sim 4$ increase compared to undiluted samples, while according to the macroscopic model given above, the presence of this small amount of microcrystals could hardly improve the mobilities. This result implies that the increased mobilities are not the direct consequence of microcrystallite formation. Instead, enhanced transport is due to the improvement in the quality of the amorphous material. This would imply narrower band tails, both at the conduction and the valence mobility edges, which results in higher drift mobilities for both electrons and holes.

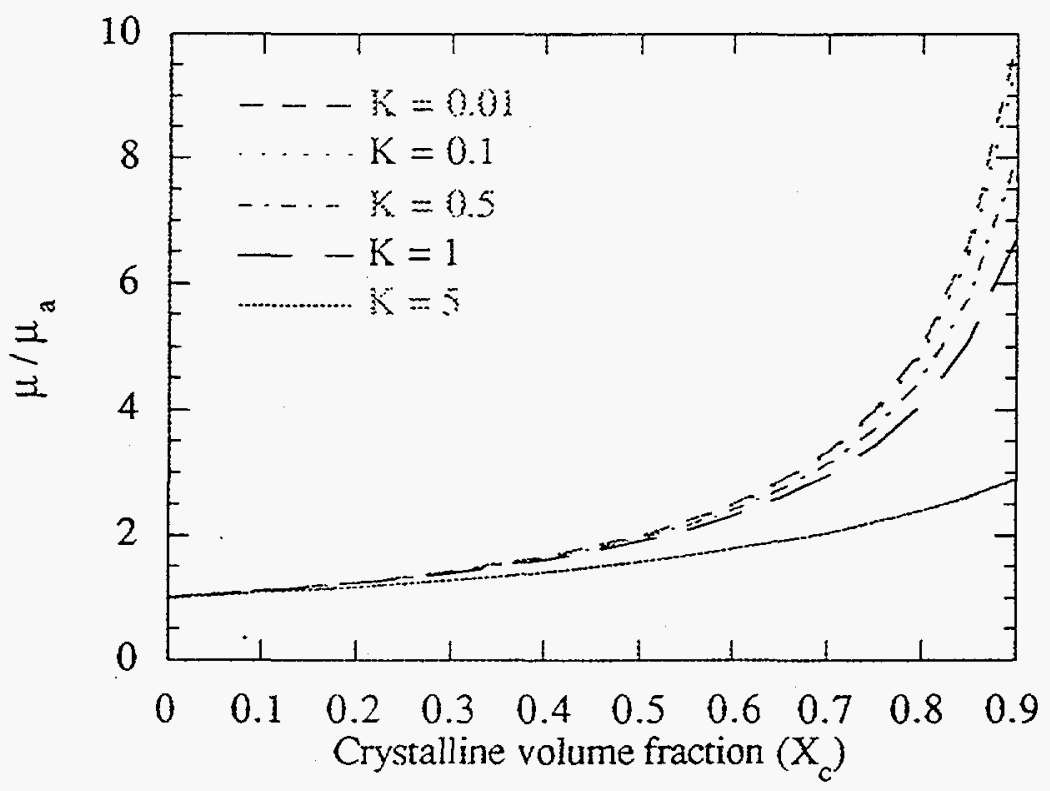

Figure 5.12. Calculation result of the effect of crystalline volume fraction on the average drift mobility in microcrystalline silicon. The average grain size was assumed to be $55 \mathrm{~nm}$. The parameter $K$ denotes the ratio of mobility in the amorphous phase to that in the boundary region. 
The increase in crystallinity by adding more hydrogen than the mixing ratio of 15 or by using higher temperature did not result in any further enhancement in mobilities. At the hydrogen / silane ratio of 20 , the crystalline fraction is $13 \sim 23 \%$, the improvements in mobilities are only by a factor of 2 or less. This further reinforces the explanation that the improvement in mobilities is due to a less disordered amorphous matrix rather than due to a presence of crystals.

(2) lifetime and $\mu \tau$ product

In a composite material, the overall lifetime of charge carriers is defined as

$$
\frac{1}{\tau}=\sum_{i} \frac{1}{\tau_{i}}
$$

If the carrier lifetime is much larger in the crystalline phase than in the amorphous matrix or in the boundary region, its contribution to the total lifetime can be ignored. Therefore,

$$
\frac{1}{\tau}=\frac{1}{\tau_{a}}+\frac{1}{\tau_{b}}
$$

From this equation, we see that, if $\tau_{b} » \tau_{a}$, i.e., if trapping in the boundary region is much smaller than that in the amorphous matrix, the overall lifetime would not be affected by the presence of phase boundaries. The lifetime in the phase boundary region is expressed as follows:

$$
\tau_{b}=\frac{1}{X_{b} v \sigma_{t} N_{t}}=\frac{D}{6 d X_{c} v \sigma_{t} N_{t}}
$$

where $v$ is the carrier drift velocity, $N_{t}$ is the trap density in the boundary region, and $\sigma_{t}$ is the capture cross section. Eqn. (5.2.9) shows that, with all the other parameters constant, the larger the crystal size or the smaller the crystal fraction, the longer the lifetime in the 
boundary. Now, a criterion is set to be $\tau_{b} \geq 10 \tau_{a}$ such that the overall lifetime can be determined solely by the lifetime in the amorphous matrix within an acceptable error bound.

Assuming the samples are biased with an electric field of $5 \times 10^{4} \mathrm{~V} / \mathrm{cm}$, which is a typical value in operating thin $(<2 \mu \mathrm{m})$ a-Si:H diodes, ranges of crystal size and volume fraction satisfying the above criterion are calculated from eqn. (5.2.9) using the following parameters: $v=5 \times 10^{4} \mathrm{~cm} / \mathrm{sec}$ for electrons and $250 \mathrm{~cm} / \mathrm{sec}$ for holes, $\tau_{a}=9 \times 10^{-8} \mathrm{sec}$ for electrons and $3 \times 10^{-6} \mathrm{sec}$ for holes, $N_{t}=2 \times 10^{18} \mathrm{~cm}^{-3}$, and $\sigma_{t}=5 \times 10^{-15} \mathrm{~cm}^{2}$. [11] The calculation results are shown in Fig. 5.13. The drawn lines divide the $D$ - $X_{\mathrm{c}}$ plane into three regions: in region $A$, neither electron nor hole lifetime is affected, in region $B$, only electron lifetime, and in $C$, both electron and hole lifetimes are affected by the captures in the phase boundaries. In other words, if the ratio of particle size (in nanometers) to crystal volume fraction is greater than 1350 , neither electron nor hole lifetimes are substantially affected by the boundary trapping, and if the ratio falls between 225 and 1350 , only electron lifetimes will be affected

The increased $\mu \tau$ values for our best sample is primarily due to the increased mobilities and apparently the lifetimes do not change in a consistent manner. However, the model analysis predicted that no appreciable effect of grain boundaries on the hole $\mu \tau$ in the range of the particle size and volume fraction values corresponding to the hydrogen / silane ratio of 20 . This is consistent with the measurement. As seen from Fig. 5.8, the hole $\mu \tau$ values for the dilution ratio of 20 did not change with deposition condition, while the crystallinity changed by a factor of 8 . 


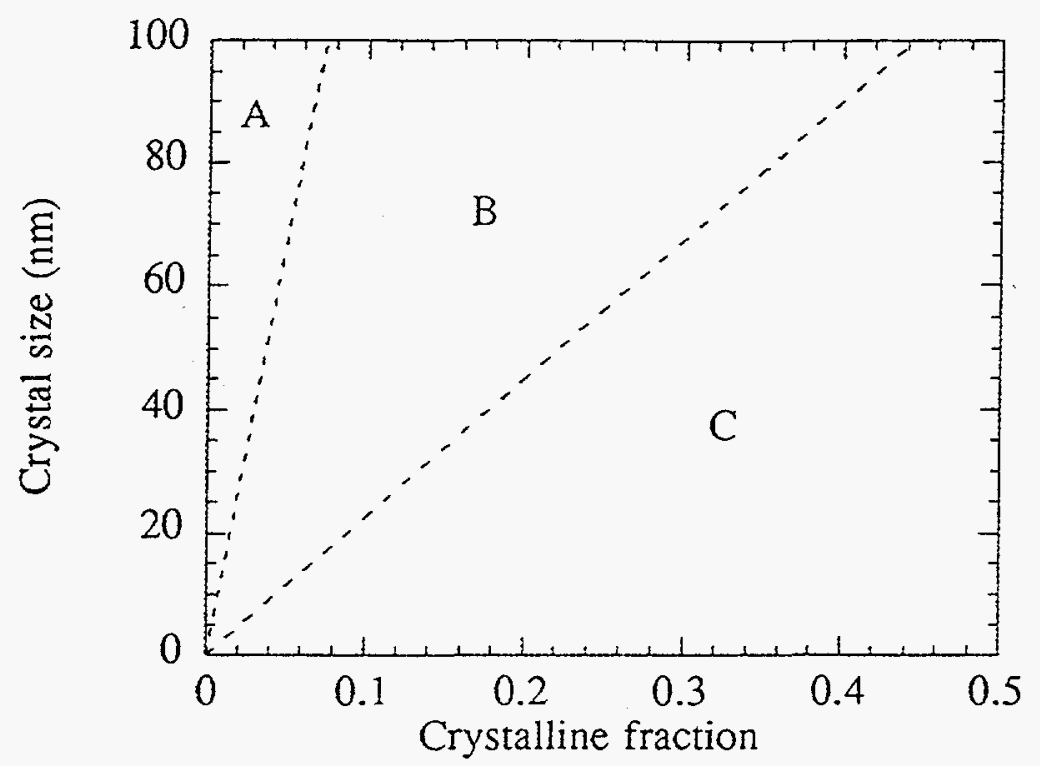

Figure 5.13. The effects of crystalline volume fraction and particle size on the carrier lifetime. In region $\mathrm{A}$, neither electron nor hole lifetimes are affected by the presence of phase boundary; In region $\mathrm{B}$ only electron lifetimes are influenced, and in region $\mathrm{C}$ both electron and hole lifetimes are affected.

Improvement in electrical parameters due to hydrogen dilution have also been reported by other groups.[21-24] They obtained improved photovoltaic characteristics in their amorphous silicon solar cells, using hydrogen dilution, both at initial and light degraded states. It is interesting that best results in this study are obtained from deposition runs with $190^{\circ} \mathrm{C}$, and most of the improved characteristics reported in references [22-24] are also at low substrate temperatures $\left(80 \sim 160^{\circ} \mathrm{C}\right)$. Apparently with hydrogen dilution, the optimum substrate temperature shifts to lower values form the conventional value of $250^{\circ} \mathrm{C}$, used for standard a-Si:H. Therefore, further investigation to explore the substrate temperature range of $100^{\circ} \mathrm{C}$ to $190^{\circ} \mathrm{C}$ should be considered. 
The measured values of ionized defect densities $\left(N_{d}{ }^{*}\right)$ are lower by a factor of $5 \sim$ 30 than those of normal amorphous silicon samples. The ionized dangling bond density, $N_{d}{ }^{*}$, from amorphous silicon samples is $\approx 0.35$ of the total dangling bond density measured by electron spin resonance (ESR) technique.[25] Therefore, the lower values of $N_{d}{ }^{*}$ for hydrogen-diluted samples may mean that, either the defect density in the amorphous part has improved by a large factor, or, for the hydrogen diluted material, the relation between the $N_{d}{ }^{*}$ as measured by hole-onset method and the total defect density, $N_{d}$, is different from the one established for standard amorphous silicon. The former may be true, if we assume that the extra hydrogen also plays a role in passivating further some of the dangling bonds in the amorphous part. This is consistent with our stress measurement results which show stress values a factor of 2 higher for our new samples, and the empirically known inverse relation between the internal stress and the dangling bond density.[26]

According to the parameters used in the model analysis, the volume fraction of the boundary region is 100 times smaller than that of the crystallites, and the boundary region contains $10^{4}$ times higher dangling bond density than the standard amorphous matrix. Therefore, for a sample having $0.1 \%$ crystallinity, due to the presence of grain boundaries, the model predicts an overall defect density about 10 times higher than the defect density of the amorphous part, while the measurement of ionized defect densities $\left(N_{d}{ }^{*}\right)$ are better by a factor of $5 \sim 30$ than that of normal amorphous silicon samples.

It should be noted that the optimum deposition condition for best quality material (hydrogen/silane ratio $=15$ ) corresponds to the onset of crystal formation. Little (< $0.02 \%$ ) or no crystallites were observed under the microscope. At this condition, the microstructure seems to have the best uniformity which is associated with good electrical transport properties and low dangling bond density. As crystallization proceeds, local ordering of atoms induces excessive strain somewhere else in the 3-dimensional network, breaking weak bonds. This was clearly demonstrated by the Xerox group using a balloon- 
and-stick model.[27] As a result microcrystalline silicon is a very porous material and its surface shows a high degree of roughness.[28] Therefore, in order to obtain high quality material, the deposition parameters must be carefully controlled so that uniform amorphous matrix and low degree of crystallinity can be manifested.

It should also be noted that the high residual stress in the hydrogen diluted material makes it difficult to make thick layers. Films thicker than $3 \mu \mathrm{m}$, deposited on $\mathrm{Cr}$-coated substrates, tend to peel off spontaneously. For this reason, the application of the H-diluted amorphous silicon is limited to thin $(<2 \mu \mathrm{m})$ devices. 


\subsection{APPLICATIONS}

Amorphous silicon has been considered as a good candidate for pixel detector / readout arrays due to the feasibility of making deposits over a large area at low cost. So far, polysilicon has been the most favored for thin film transistor (TFT) applications because of its superior electrical properties. Polysilicon layers for simple switching devices are in use industrially for fax machine heads and other devices. However, usually a high temperature $\left(\sim 900^{\circ} \mathrm{C}\right)$ is required to obtain good quality polysilicon layers. The high temperature processing limits the choice of substrate materials and other components. Therefore, it is important to improve the electrical quality of a-Si:H which can be prepared at substantially low temperatures. $\left(<250^{\circ} \mathrm{C}\right)$ The hydrogen diluted material is more suitable than conventional a-Si:H for TFT applications because of its higher electrical transport properties. In this section, plausible applications of high quality amorphous silicon will be suggested, and relative merits of amorphous and polycrystalline silicon will be discussed.

Two-dimensional arrays of detector diodes and readout electronics are shown schematically in Fig. 5.14. In Fig. 5.14(a), each element is read out line by line by sending out a signal to the a-Si:H switching transistor in that line. The charge in the pixels in the tagged line can be read out by some integrated circuit chip with multiple inputs such as the SVX chip developed at the Lawrence Berkeley Laboratory.[29] Alternative readout methods involve a single diode connected to each element (Fig. 5.14(b)) or two diodes (Fig. 5.14(c)). The two diode readout is faster and has a larger dynamic range than the single diode scheme.[30] It also minimizes switching transients. Diode readouts have the following advantage compared to TFT; (a) they require fewer number of production masks, (b) they minimize radiation damage problems as $\mathrm{SiO}_{2}$ or $\mathrm{Si}_{3} \mathrm{~N}_{4}$ gate insulators are not needed.[30] Figure 5.15 shows the structure of a-Si:H TFT, polysilicon TFT and a cross section of the diode readout. 


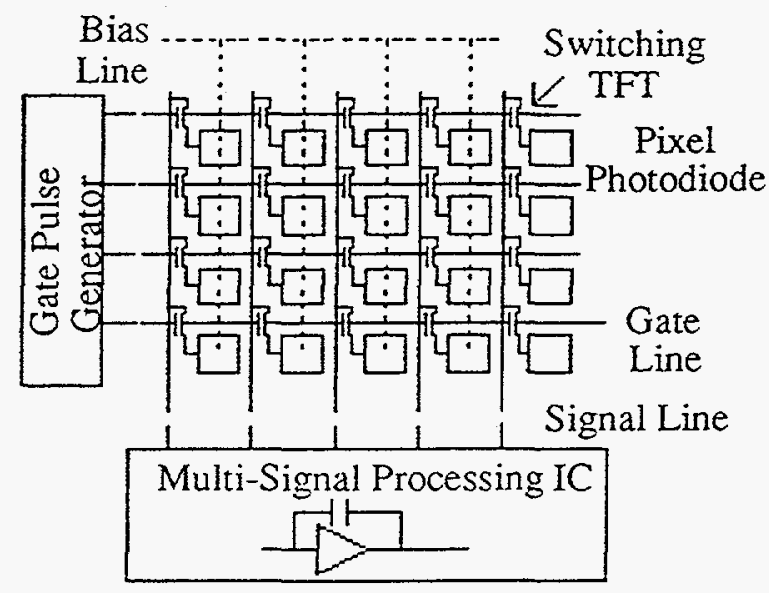

(a)

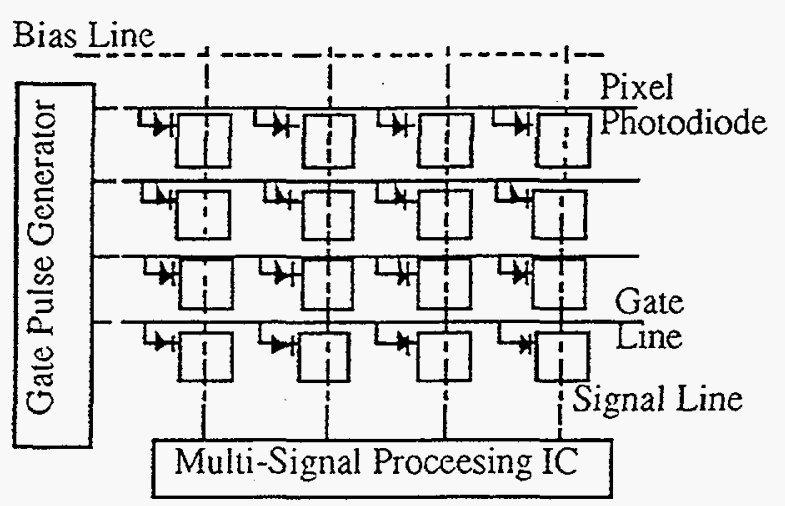

(b)

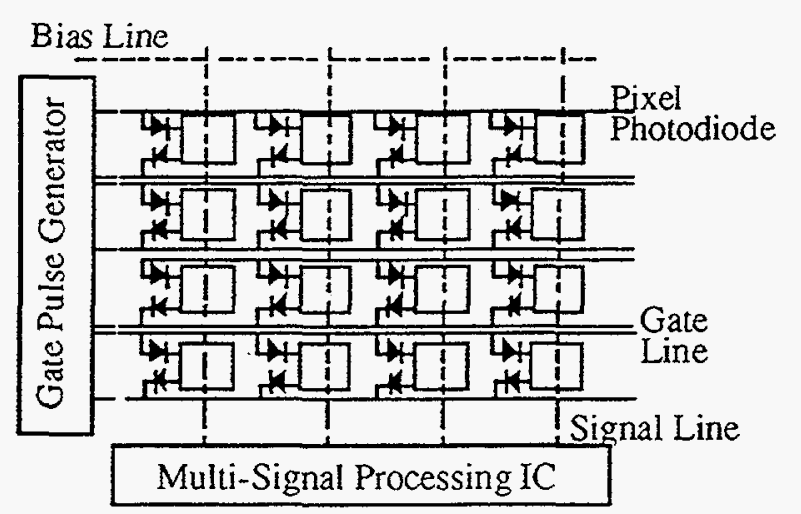

(c)

Figure 5.14. 2-dimensional readout schemes: (a) TFT, (b) single diode, and (c) two diode switches. 


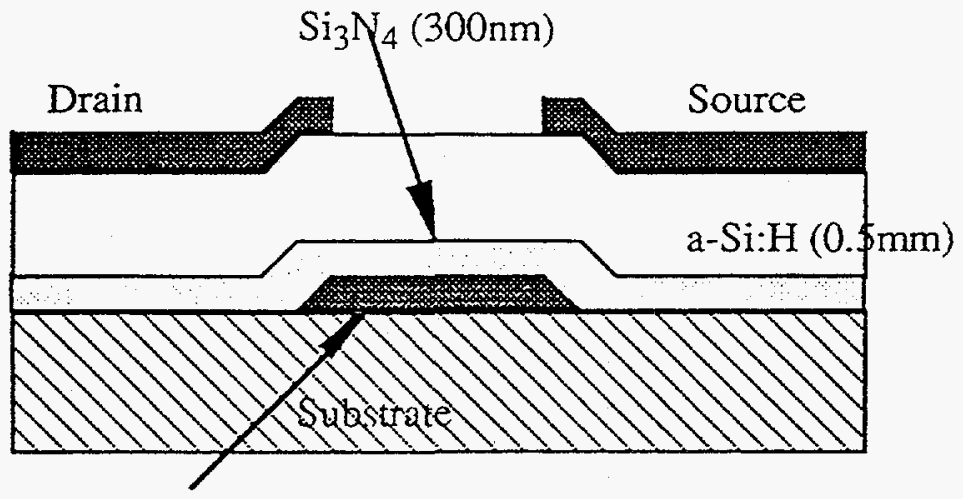

Gate (Cr)

(a)

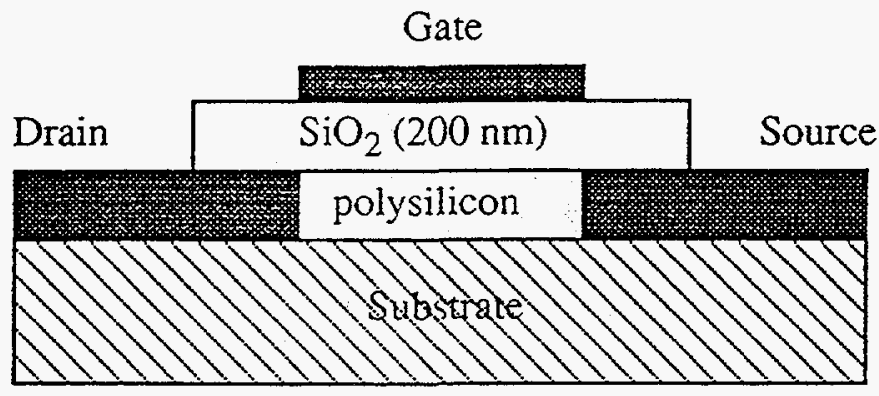

(b)

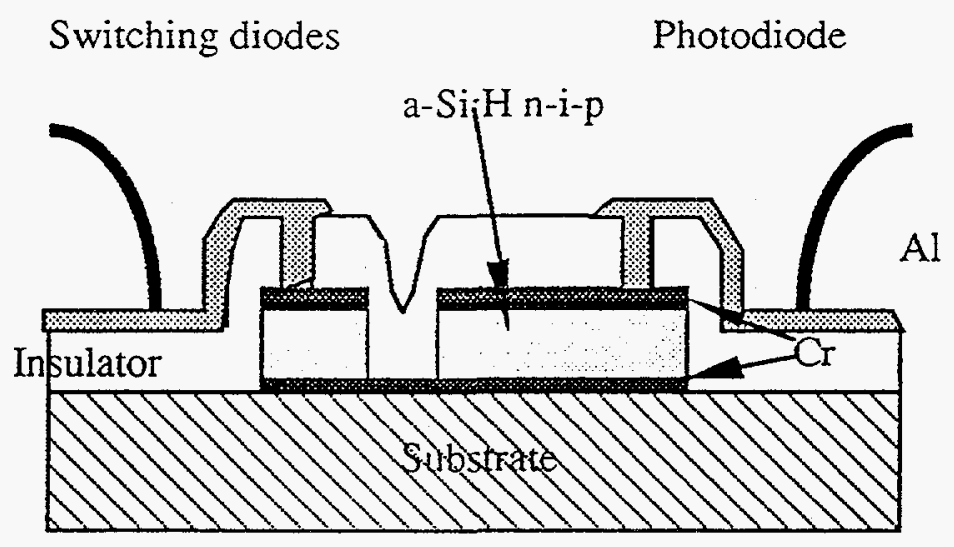

(c)

Figure 5.15. Structure of TFTs and diode readout (a) a-Si:H TFT, (b) polysilicon TFT, (c) diode readout cross section 
Table V-3. Typical properties of a-Si:H and polysilicon TFTs

\begin{tabular}{ccc}
\hline Property & a-Si:H TFT & Poly-Si TFT \\
\hline$\mu_{\mathrm{e}}\left(\mathrm{cm}^{2} /\right.$ vsec $)$ & 1 & 150 \\
$\mu_{\mathrm{h}}\left(\mathrm{cm}^{2} / \mathrm{Vsec}\right)$ & 0.005 & 80 \\
$\mathrm{~g}_{\mathrm{m}}(\mu \mathrm{A} / \mathrm{V})$ & 5 & 150 \\
Bandwidth $(\mathrm{MHz})$ & 5 & 100 \\
Noise* $\left.^{*}\right)$ & 500 & 500 \\
\hline
\end{tabular}

*Equivalent noise charges for a $\mathrm{CR}-(\mathrm{RC})^{4}$ shaping time of $1 \mu \mathrm{sec}$.

Two dimensional arrays of a-Si: $\mathrm{H}$-i-n diodes are good candidates for $\beta$-radiochromatography applications. A radio-chromatogram consists of a column along which separation of components of a labeled mixture is possible by detecting the position and concentration of a series of spots or bands produced by accumulation of different component. Normally, $\beta$-emitters such as ${ }^{3} \mathrm{H}\left(E_{\beta \max }=18 \mathrm{KeV}\right),{ }^{14} \mathrm{C}\left(E_{\beta \max }=150 \mathrm{KeV}\right)$, and ${ }^{32} \mathrm{P}\left(\mathrm{E}_{\beta \max }=1.7 \mathrm{MeV}\right)$ are used for labeling the mixtures. In a two dimensional scheme, a number of mixtures can be analyzed simultaneously, by using more columns on a chromatogram. For a real time analysis, a-Si:H pixel arrays have possible advantages of operational simplicity compared to the alternative systems such as multiwire proportional chambers (MWPC).[31]

Thermal neutrons can also be detected by a-Si:H p-i-n diodes coupled to gadolinium (Gd) converters which emit an $80 \mathrm{keV}$ internal conversion line by neutron capture. Such detectors were developed by evaporating $\sim 2 \mu \mathrm{m}$ thick Gd films on $20-30 \mu \mathrm{m}$ thick a-Si:H diodes.[32] Using a sandwiched structure with two layers of enriched Gd film in the ${ }^{157} \mathrm{Gd}$ isotope coupled to $\mathrm{p}-\mathrm{i}-\mathrm{n}$ diodes, thermal neutron efficiencies of more than $60 \%$ can be obtained. Such detectors in a two dimensional configuration are excellent candidates for 
applications where high spatial resolution and good thermal neutron sensitivity with high gamma rejection are desirable.

There are some applications that require amplification of the collected signal to bring it to a satisfactory level above the system noise. This can be accomplished by introducing a TFT amplifier made of polysilicon underneath each element of the array, and then reading the output by enabling lines as in the diode case. Amorphous silicon TFT can only be used as switching devices because the threshold voltage of the gate bias shifts continuously under dc bias.[33] True CMOS amplifiers can be made using polysilicon TFT since both electron and hole mobilities are comparable and much higher than the corresponding ones for a-Si:H TFT. Table V-3 summarizes electronic characteristics of amorphous and polycrystalline silicon TFTs. Fabrication of polysilicon TFTs with high mobility and uniform microstructure in a low temperature process $\left(<600^{\circ} \mathrm{C}\right)$ has been studied by various approaches for applications to LCDs and 2-dimensional image sensors. Recently, a field effect mobility as high as $440 \mathrm{~cm}^{2} / \mathrm{Vs}$, which is only about a half of that in single crystalline silicon, has been accomplished by an excimer laser annealing method.[34]

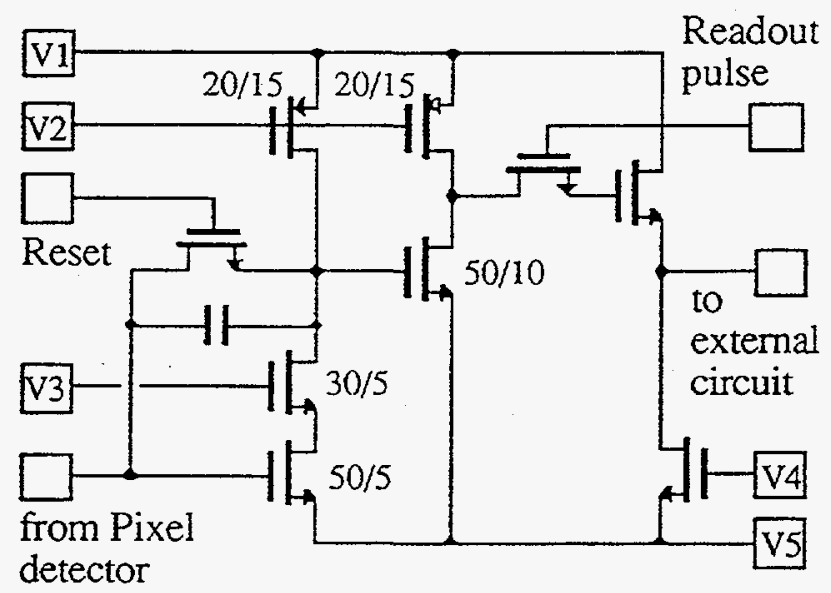

Figure 5.16. Schematic circuit diagram of the prototype polysilicon TFT charge sensitive pixel amplifier for a-Si:H pixel detectors. Each square with node numbers represents a test pad. $\mathrm{N}$ and $\mathrm{P}$ stands for $\mathrm{n}$ - and $\mathrm{p}$-channel TFTs, respectively, and $\mathrm{C}$ for a capacitor.[35] 
Using the facilities of the Xerox Palo Alto Research Center, 3-stage CMOS polysilicon amplifiers were designed and tested.[35] The amplifiers consist of a charge sensitive input stage, a voltage gain stage, and a low impedance output stage for driving signals through a pixel array. This prototype amplifier design is shown in Fig. 5.16. The gain-bandwidth product of the amplifier is $\sim 350 \mathrm{MHz}$. When the amplifier is connected to a pixel detector with a capacitance of $0.2 \mathrm{pF}$, it gives a charge-voltage gain of $\sim 0.02$ $\mathrm{mV} / \mathrm{electrons}$ with a pulse rise time less than $100 \mathrm{nsec}$. An equivalent noise charge of the front-end TFT is $\sim 1000$ electrons for a shaping time of $1 \mu \mathrm{sec}$.

The noise produced in a reverse biased $\mathrm{p}-\mathrm{i}-\mathrm{n}$ detector together with that of a typical readout amplifier should be small. In Fig. 5.17, the noise in a $26 \mu \mathrm{m}$ diode as a function of reverse bias, measured by a charge sensitive amplifier with 2.5 and $0.5 \mu \sec C R-(R C)^{2}$ shaping times. The flat portion of the noise graph, at low biases, is the sum of the amplifier noise when loaded by the capacity of the detector and a mostly resistive (Nyquist) noise generated by the contact resistance and the p layer resistance. At higher biases when the reverse current increases, the contributing shot noise which has a flat frequency spectrum and is proportional to the current is observed. At still higher biases with larger reverse current, $1 / f$ noise, which has the $1 / f$ spectral response and is proportional to $I^{2}$ becomes predominant. All of these noise components are proportional to the area of a pixel detector.[36, 37]

The signal response as a function of shaping time can be calculated from the mobility and the electric field in the i-layer at a given bias. Figure 5.18 shows the signal from electrons and holes for thin $(1 \mu \mathrm{m})$ detector diodes made by conventional method and by hydrogen dilution, respectively. The parameters used in this calculation are: $\mu_{e}=1$ $\mathrm{cm}^{2} / \mathrm{V} \cdot \mathrm{s}, \mu_{h}=0.005 \mathrm{~cm}^{2} / \mathrm{N} \cdot \mathrm{s}, \tau_{e}=9 \times 10^{-8} \mathrm{sec}, \tau_{h}=3 \times 10^{-6} \mathrm{sec}$, for standard a-Si:H, and $\mu_{e}=4 \mathrm{~cm}^{2} / \mathrm{V} \cdot \mathrm{s}, \mu_{h}=0.01 \mathrm{~cm} / \mathrm{V} \cdot \mathrm{s}, \tau_{e}=5 \times 10^{-8} \mathrm{sec}, \tau_{h}=5 \times 10^{-6} \mathrm{sec}$, for hydrogendiluted samples. Fast timing for both is achieved by collecting the full electron signal only 
in $<5$ and 0.08 nsec., respectively. Since both the mobility and lifetime are larger in the hydrogen-diluted diode, the shaping time for full charge collection can be greatly reduced.

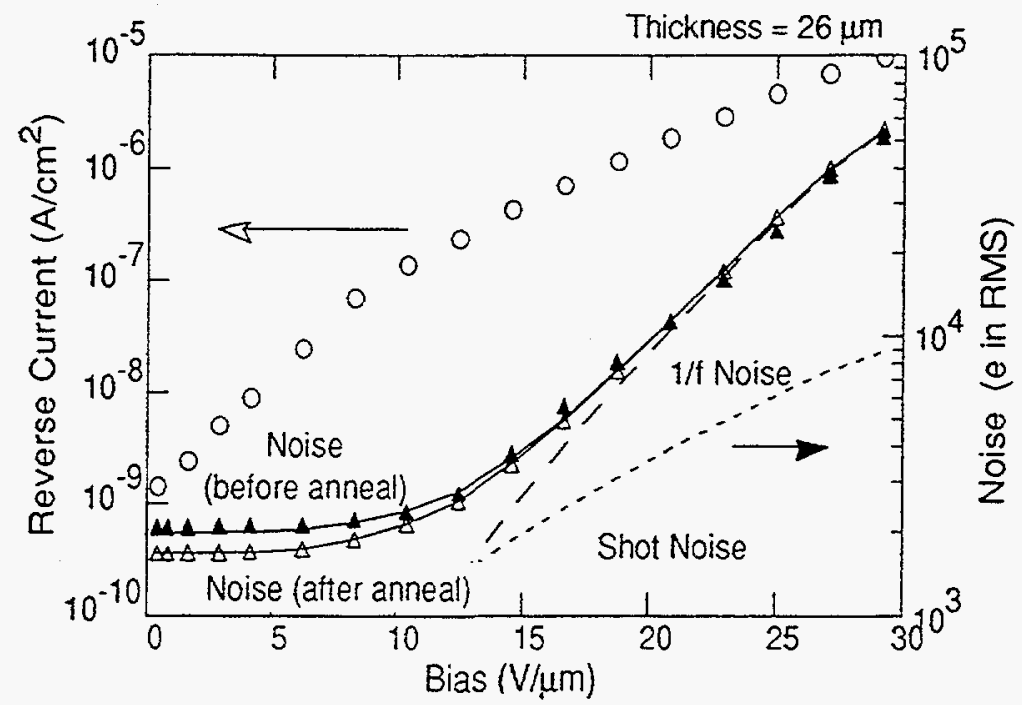

(a)

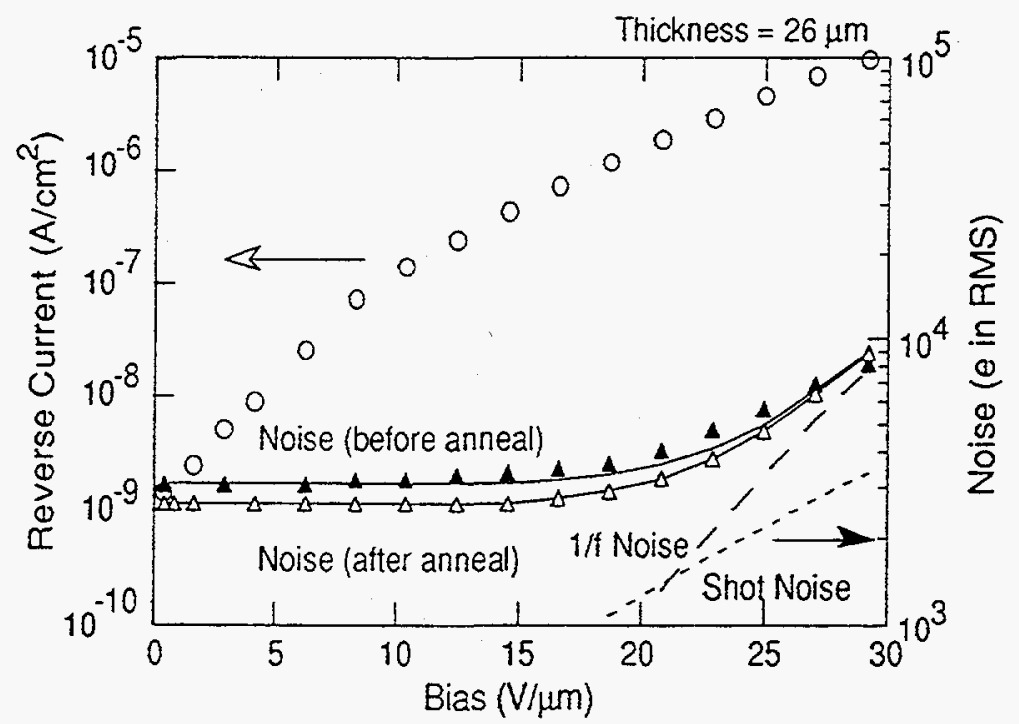

(b)

Figure 5.17. Resistive, shot and $1 / \mathrm{f}$ noise in a-Si:H p-i-n detector diode at a shaping time of (a) $2.5 \mu \mathrm{sec}$. and (b) $0.5 \mu \mathrm{sec}$. 


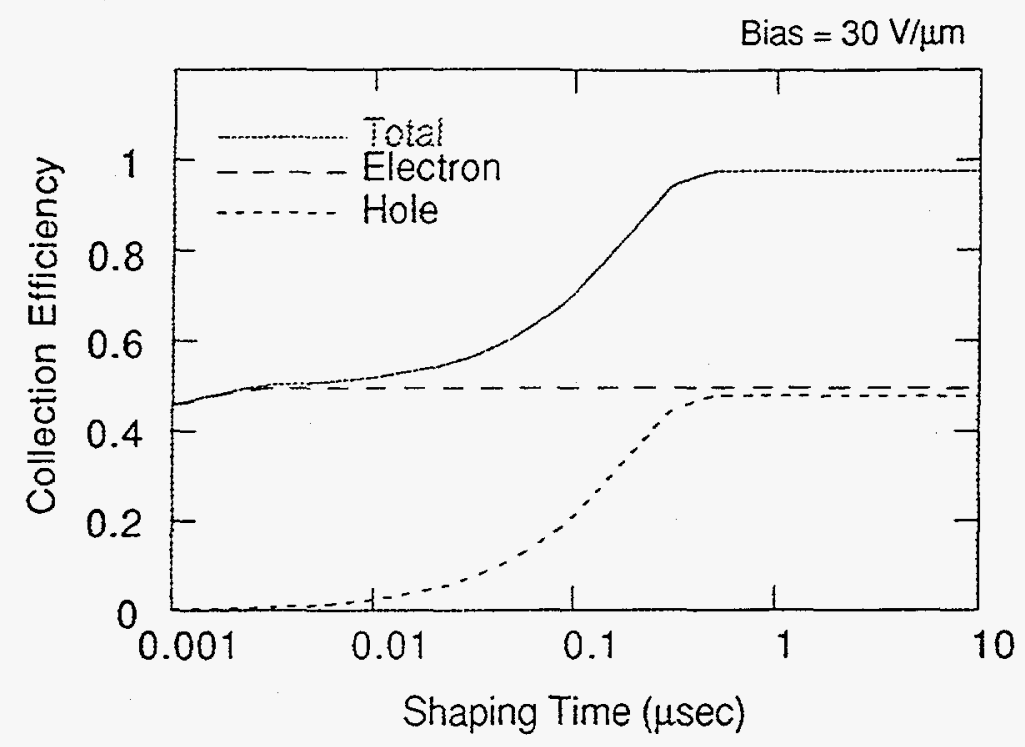

(a)

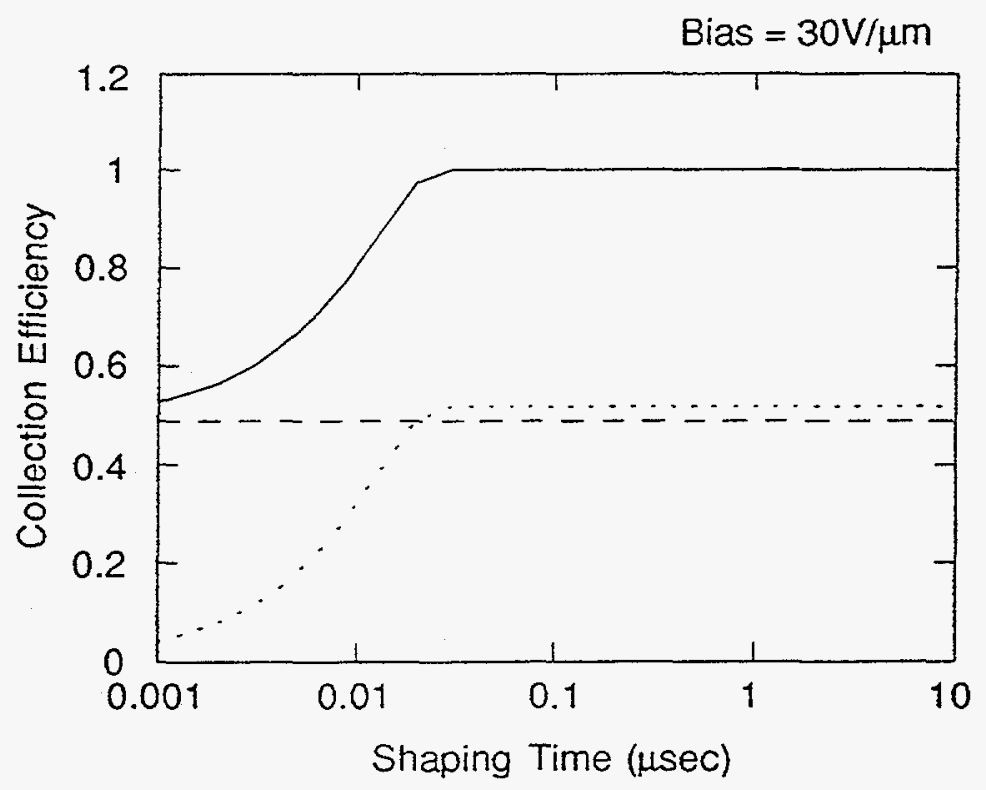

(b)

Figure 5.18. Normalized electron and hole collection efficiency for $1 \mu \mathrm{m}$ thick p-i-n diodes: (a) standard a-Si:H (b) hydrogen-diluted sample 


\subsection{SUMMARY}

The effects of hydrogen dilution of silane on various material properties of a-Si:H films were studied. For the hydrogen-diluted samples, measurements on electron and hole $\mu, \mu \tau$, and $N_{d}{ }^{*}$ have been performed. Microcrystals were formed when the hydrogen to silane gas flow ratio is 20 or higher. Hydrogen dilution enhanced the electronic properties of a-Si:H films at the expense of high internal stress. The optimum deposition parameters were found to be the hydrogen dilution ratio of 15 , substrate temperature of $190^{\circ} \mathrm{C}$, and $\mathrm{RF}$ power density of $60 \mathrm{~mW} / \mathrm{cm}^{2}$. Under these conditions, enhancement of carrier mobilities by factors of $3 \sim 4$ and $\mu \tau$ values by a factor of 2 , compared to the standard a-Si:H, was achieved. The ionized dangling bond density was also reduced by a factor of 10 , and thus full depletion of the detector could be achieved at a lower bias voltage. A simple model to analyze the effect of microcrystals and their grain boundaries were presented, and the results were consistent with the measurements. According to the model, the improvements in electrical properties are due to the following roles of extra hydrogen: a) narrowing the band tails by breaking loose $\mathrm{Si}-\mathrm{Si}$ bonds and therefore bringing about more long range order; b) passivating additional dangling bonds in the amorphous material.

With higher carrier mobility in the intrinsic layer, detectors made of hydrogendiluted material will have a faster response. Therefore, the H-diluted material is advantageous for use in large area two-dimensional arrays, in which detector pixels can be integrated with TFT switches deposited by the same process and even read out by such TFT amplifiers. This is also a crucial technology for flat screen displays. 


\section{References}

[1] S.A.Kleinfelder, W.C.Carithers, R.P.Ely, C.Haber, F.Kirsten and H.G.Spieler, IEEE Trans. Nucl. Sci., NS-35 171 (1988)

[2] E.H.M.Heijne and P.Jarron, Nucl. Instr. Meth., A 275467 (1989)

[3] J.E.Bateman, Nucl. Instr. Meth., A 273721 (1988)

[4] V.Perez-Mendez, J.drewery, W.S.Hong, T.Jing, S.N.Kaplan, H.Lee and A.Mireshghi, Presented at the Electrochem. Soc. Thin Film Transistor Tech. Symp., Miami, FL, Oct. 9-14, 1994

[5] T.Jing, C.A.Goodman, G.Cho, J.S.Drewery, W.S.Hong, H.Lee, S.N.Kaplan, A.Mireshghi, V.Perez-Mendez and D.Wildermuth, IEEE Trans. Nucl. Sci., NS-41 4 903 (1994)

[6] S. Veprek and V. Maracek, Solid State Electronics, 11, 683 (1968)

[7] Y.Uchida, T.Ichimura, M.Ueno and H.Heruki, Jap. J. Appl. Phys., 21, L586 (1982)

[8] Y. Matsumoto, G. Hirata, H. Tkakura, H. Okamoto, and Y. Hamakawa, J. Appl. Phys., 67, 6536 (1990)

[9] L. Yang, L. Chen, S. Wiedeman and A. Catalano, Mat. Res. Soc. Symp. Proc., 283, 463 (1993)

[10] S.S.He, M.J.Williams, D.J.Stephens and G.Lucovsky, J. Non-Cryst. Sol., 1641661263 (1993)

[11] R.A.Street, "Hydrogenated Amorphous Silicon", Cambridge Univ. Press, (1991)

[12] A. Matsuda, in Amorphous Semiconductor Technologies and Devices, Ed. Y. Hamakawa, JARECT 22, pp. 111 OHMSHA and North Holland (1987)

[13] Y. Mishima, S. Miyazaki, M. Hirose and Y. Osaka, Phil. Mag. B, 46, 31 (1982)

[14] K.P. Chik, P. H. Chan, B. Y. Tong, S. K. Wong and P. K. John, Phil. Mag. B, 61, $377(1990)$

[15] A.C. Hourd, D. L. Melville and W. E. Spear, Phil. Mag. B, 64, 533 (1991) 
[16] B.D.Cullity, "Elements of X-ray Diffraction", Addison-Wesley, Reading (1956)

[17] B.E.Warren, Progress in Metal Physics, 8147 (1959)

[18] D.A.Porter and K.E.Easterling, "Phase Transformations in Metals and Alloys", pp.186, Van Nostrand Reinhold Co. (1981)

[19] S.Ishihara, D.He, T.Akasaka, Y.Araki, M.Nakata and I.Shimizu, Mat. Res. Soc. Symp. Proc., 283489 (1993)

[20] K.S.Stevens and N.M.Johnson, J. Appl. Phys., $71 \underline{6} 2628$ (1992)

[21] P.K.Acharya, H.D.Banerjee, K.L.Chopra, S.C.Saha and S.Ray, Solar Energy Mater. \& Solar Cells, 3221 (1994)

[22] Y.Hishikawa, S.Tsuge, N.Nakamura, S.Tsuda, S.Nakano and Y.kuwano, J. Appl. Phys., 69508 (1991)

[23] Y.Hishikawa, M.Sasaki, S.Tsuge, S.Okamoto and S.Tsuda, Mat. Res. Soc. Symp. Proc., 297779 (1993)

[24] J.Yang, X.Xu and S.Guha, To be published in Mat. Res. Soc. Symp. Proc. (1994).

[25] S.Qureshi, V.Perez-Mendez, S.N.Kaplan, I.Füieda, G.Cho and R.A.Street, Mat. Res. Soc. Symp. Proc., 149649 (1989)

[26] W.S.Hong, V.Petrova-Koch, J.Drewery, T.Jing, H.Lee and V.Perez-Mendez, Presented in Mat. Res. Soc. Spring Meeting, San Francisco, April 17-21, 1995, and to be published in the proceedings.

[27] R.A.Street, Private communication

[28] S.Kumar, B.Drevillon and C.Godet, J. Appl. Phys., 601542 (1986)

[29] S.A.Kleinfelder, W.C.Carithers, R.P.Ely, C.Haber, F.Kirsten, H.Spieler, IEEE Trans. Nucl. Sci., NS-35 171 (1988)

[30] G.Cho, J.S.Drewery, W.S.Hong, T.Jing, H.Lee, S.N.Kaplan, A.Mireshighi, V.Perez-Mendez and D.Wildermuth, Mat. Res. Soc. Symp. Proc., 297 969-974 (1993)

[31] H.Filthuth, J. Planar Chromatography, 2198 (1989) 
[32] A.Mireshghi, G.Cho, J.S.Drewery, W.S.Hong, T.Jing, H.Lee, S.N.Kaplan and V.Perez-Mendez, IEEE Trans. Nucl. Sci., NS-41 4915 (1994)

[33] W.B.Jackson and M.D.Moyer, Phys. Rev., B36 6217 (1987)

[34] H.Kuriyama, T.Kuwahara, K.Wakisaka, S.Kiyama, S.Tsuda and S.Nakano, Presented at the 186th Electrochem. Soc. Thin Film Tech. Symp., Miami, Florida, Oct. 9-14, 1994

[35] G.Cho, V.Perez-Mendez, M.Hack and A.Lewis, Mat. Res. Soc. Symp. Proc., 258 1181 (1992)

[36] J.Xi, R.E.Hollingsworth, R.H.Buitrago, D.Oakley, J.P.Cumalat, U.Nauenberg, J.A.McNeil, D.F.Anderson and V.Perez-Mendez, Nucl. Instr. Meth., A301 219 (1991)

[37] G.Cho, J.S.Drewery, W.S.Hong, T.Jing, S.N.Kaplan, H.Lee, A.Mireshghi, V.Perez-Mendez and D.Wildermuth, IEEE Trans. Nucl. Sci., NS-40 323 (1994) 


\section{CHAPTER 6. CONCLUSION}

Hydrogenated amorphous silicon and related materials have been applied to radiation detectors, utilizing their good radiation resistance and feasibility of making deposits over a wide area at low cost. Effects of growth parameters, source gas, postdeposition heat-treatment, alloying and doping on various material properties of a-Si:H have been studied to satisfy the requirements for specific detection application.

Thick $(\sim 50 \mu \mathrm{m})$ a-Si:H p-i-n diodes for direct detection of minimum ionizing particles have been prepared with low internal stress by deposition at low temperature from $40 / 60 \mathrm{He}^{-\mathrm{SiH}_{4}}$ mixture in order to minimize substrate bending and delamination. A moderate increase in the growth rate to $3.5 \sim 4 \mu \mathrm{m} / \mathrm{hr}$. and residual stress as low as $\sim 80$ $\mathrm{MPa}$ have been obtained. Lowering the deposition temperature from $250^{\circ} \mathrm{C}$ to $150^{\circ} \mathrm{C}$ reduced the residual stress by a factor of $\sim 4$, but at the same time the ionized dangling bond density jumped from $5 \times 10^{14} \mathrm{~cm}^{-3}$ to $2.5 \times 10^{15} \mathrm{~cm}^{-3}$. The increased $N_{d}^{*}$ was brought to a sufficiently low value $\left(7 \times 10^{14} \mathrm{~cm}^{-3}\right)$ by post-annealing at $160^{\circ} \mathrm{C}$ for 100 hours. The combination of the low temperature growth, He-dilution and post annealing of the film lent a way to produce a material that has a low residual stress and electrical properties satisfactory for charged particle detectors. The structure of the new film contained voids and tiny crystalline inclusions and was different from the one observed in conventional aSi:H. He-diluted material is good for thick diodes for direct detection of charged particles due to the high deposition rate and low ionized defect density.

Deposition on patterned substrates was attempted as an alternative to controlling deposition parameters to minimize substrate bending and delamination of thick a-Si:H films. Growth on an inverted-pyramid pattern of $200 \mu \mathrm{m}$ in lateral dimension reduced the substrate bending by a factor of $3 \sim 4$ for the same thickness film. The inverted-pyramid pattern had additional advantages such as increased effective thickness for signal generation and reduced electron-hole recombination during transit. 
Thin $(0.1 \sim 0.2 \mu \mathrm{m})$ films of a-Si:H and a-Si:C:H have been applied to microstrip gas chambers to control gain instabilities due to charges on the substrate. Light sensitivity of the a-Si:H sheet resistance was reduced to a great extent by alloying with carbon and further reduced to nearly unity by boron doping. The surface resistivity of the resulting ptype a-Si:C:H was successfully controlled in the range of $10^{12} \sim 10^{17} \Omega / \square$ by changing the carbon content and boron doping density in the film. The p-doped a-Si:C:H also showed shorter equilibration time to reach a stable resistance value than the undoped a-Si:H. Performance of the detectors with a-Si:C:H layer was comparable to that of electronicconducting glass. Upon prolonged irradiation, the detector having p-doped a-Si:C:H layer overcoated on the microstrip structure aged more slowly compared to the one built on a bare glass substrate.

Thick $(4.6 \mu \mathrm{m})$ undoped a-Si: $\mathrm{C}: \mathrm{H}$ having high resistivity and good dielectric strength have been prepared from $10 / 90 \mathrm{SiH}_{4}-\mathrm{CH}_{4}$ mixture and used as a spacer layer between the anodes and cathodes in microgap chamber. These results show that an amorphous silicon-carbon alloy finds applications to essential components in micro-scale gas-filled detectors, due to the ease with which large area may be covered, the wide range of surface resistivities possible and the low cost of fabrication.

Hydrogen dilution of silane has been explored to improve electrical transport properties of a-Si:H material for high speed photodetectors and TFT applications. For hydrogen-diluted material, electrons and hole mobilities $\sim 4$ times larger and mobilitylifetime product $2 \sim 3$ times greater than those of conventional a-Si:H have been achieved. The density of ionized dangling bonds also showed a factor of $5 \sim 10$ improvement at the expense of increased residual stress. The optimal material characteristics for TFT application appeared at deposition conditions corresponding to the onset of crystalline formation, indicating that the enhancement of the electrical properties is due to the superior amorphous matrix not to the presence of crystalline phase. Owing to its higher carrier mobilities, the hydrogen diluted material is advantageous for use in large are 2-dimensional 
arrays, in which detector pixels can be integrated with TFT switches deposited by the same process.

Material properties of the a-Si:H based materials can be easily adapted to the characteristics required for individual applications by modifying fabrication conditions and procedures. This asset, in addition to the advantages of large area capability and radiation hardness, makes a-Si:H an attractive candidate for radiation detection applications. 